\title{
Dynamic Multiscale Modeling of Materials at the Nanoscale
}

\author{
by \\ Behrouz Shiari

\begin{abstract}
A thesis submitted to the
Faculty of Graduate Studies and Research

in partial fulfillment of the requirements for the Degree of

Doctor of Philosophy
\end{abstract}

Ottawa-Carleton Institute for Mechanical and Aerospace Engineering

Department of Mechanical and Aerospace Engineering

Faculty of Engineering

Carleton University

Ottawa, Canada

September 2008

(C) Behrouz Shiari, 2008 


$\begin{array}{ll}\begin{array}{l}\text { Library and } \\ \text { Archives Canada }\end{array} & \begin{array}{l}\text { Bibliothèque et } \\ \text { Archives Canada }\end{array} \\ \begin{array}{l}\text { Published Heritage } \\ \text { Branch }\end{array} & \begin{array}{l}\text { Direction du } \\ \text { Patrimoine de l'édition }\end{array} \\ \begin{array}{l}\text { 395 Wellington Street } \\ \text { Ottawa ON K1A 0N4 } \\ \text { Canada }\end{array} & \begin{array}{l}\text { 395, rue Wellington } \\ \text { Ottawa ON K1A 0N4 } \\ \text { Canada }\end{array}\end{array}$

Your file Votre référence ISBN: 978-0-494-47491-4 Ourfile Notre référence ISBN: 978-0-494-47491-4

NOTICE:

The author has granted a nonexclusive license allowing Library and Archives Canada to reproduce, publish, archive, preserve, conserve, communicate to the public by telecommunication or on the Internet, loan, distribute and sell theses worldwide, for commercial or noncommercial purposes, in microform, paper, electronic and/or any other formats.

The author retains copyright ownership and moral rights in this thesis. Neither the thesis nor substantial extracts from it may be printed or otherwise reproduced without the author's permission.
AVIS:

L'auteur a accordé une licence non exclusive permettant à la Bibliothèque et Archives Canada de reproduire, publier, archiver, sauvegarder, conserver, transmettre au public par télécommunication ou par l'Internet, prêter, distribuer et vendre des thèses partout dans le monde, à des fins commerciales ou autres, sur support microforme, papier, électronique et/ou autres formats.

L'auteur conserve la propriété du droit d'auteur et des droits moraux qui protège cette thèse. $\mathrm{Ni}$ la thèse ni des extraits substantiels de celle-ci ne doivent être imprimés ou autrement reproduits sans son autorisation.
In compliance with the Canadian Privacy Act some supporting forms may have been removed from this thesis.

While these forms may be included in the document page count, their removal does not represent any loss of content from the thesis.
Conformément à la loi canadienne sur la protection de la vie privée, quelques formulaires secondaires ont été enlevés de cette thèse.

Bien que ces formulaires aient inclus dans la pagination, il n'y aura aucun contenu manquant.

\section{Canada}




\begin{abstract}
Dynamic coupling of atomistic and continuum computational methods of solids at the nanoscale have received much interest recently, because many problems are not addressed well by either model alone. In the dynamic multiscale approaches, much emphasis has been placed on damping spurious reflections at the interface of the coupled regions. We have revised and extended an existing static coupled method to a dynamic concurrent method at finite temperature in which a damping band (or thermostating zone) minimizes spurious reflections. The coupled formulation is composed of a continuum region (modeled via Finite element) and molecular dynamics in a atomistic region. Our investigation on $1 D$ test simulations of the method clearly shows that the damping band not only dissipates the reflected wave energy from the atomistic/continuum interface but also the energy of waves which are reflected off the $F E$ mesh as it coarsens away from the atomistic region.

The developed method has the ability of treating dislocations as either atomistic or continuum entities within a single computational framework at finite temperature. The method captures, at the same time, the atomistic mechanisms and the long-range dislocation effects without the computational cost of full atomistic simulations. This multiscale model is found to be an effective system for both visualization and quantification of dislocation nucleation and motion, including the deformation of the atomistic region resulting from defect nucleation. We have conducted simulations to study several applications such as nanoindentation, nanoscratching and nanomachining. The results reported here provide direct and fundamental mechanistic insights into the processes associated with contact deformation and quantitative predictions of discrete plasticity events that are consistent with experimental observations.
\end{abstract}




\section{Acknowledgements}

This research couples traditional Solid mechanics, Physics and Materials Science together. I am really been forced to extend my knowledge beyond my previous understanding and training as a mechanical engineer to finish this research. I have been accompanied and supported by many people and it is now a pleasant opportunity to express my gratitude to all of them.

First, I deeply indebted to my supervisor Professor Ronald E. Miller whose wisdom, guidance, and encouragement helped me to successfully finish this research work. I would like to thank for his endless brightness for every problem we encoutered and for his insistence on excellence and unwillingness to settle along the way. It was a pleasure and an honor to work with him. Without him this work would never have been.

I would like to give my special thanks to my wife Flora for the inspiration, love and moral support she provided throughout my research work. Without her loving support and understanding I would never have completed my work. I also gratefully acknowledge the cooperation that I received from other faculty members and administrative staff of my department. 
To my wife, Flora 


\section{Contents}

Abstract ............................. iii

Acknowledgements $\ldots \ldots \ldots \ldots \ldots \ldots$ iv

Table of Contents $\ldots \ldots \ldots \ldots \ldots$ vi

List of Figures $\ldots \ldots \ldots \ldots \ldots \ldots \ldots \ldots \ldots$ ix

List of Tables $\ldots \ldots \ldots \ldots \ldots \ldots \ldots \ldots \ldots \ldots \ldots \ldots \ldots$

1 Introduction. 1

1.1 Simulation of Solids at Different Size/Time-scales . . . . . . . . . . . 2

1.2 Classification of Multiscale Methods . . . . . . . . . . . . . 3

1.3 Concurrent Coupling of Atomistic-Continuum . . . . . . . . . . 5

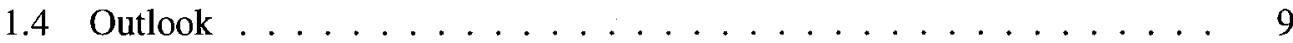

1.5 Simplifying Assumptions $\ldots \ldots \ldots \ldots$

2 Basic Concept of Concurrent Multiscale Model at Finite Temperature: 1D Verification

2.1 General Field Equations of Atomistic and Continuum Regions . . . . . 13

2.1.1 Molecular Dynamics Method ................ 14

2.1.2 Modeling Issues in Constant Temperature MD f . . . . . . 18

2.1 .3 Finite Element Method . . . . . . . . . . . . . . . . 25

2.2 Concurrent Coupling Procedure and the Dynamic Solver . . . . . . . 28

2.3 Implementation and Numerical Examples . . . . . . . . . . . . 33 
2.3.1 Coupling between a harmonic lattice and a linear FE discrete con-

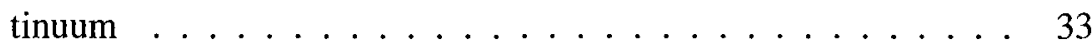

2.3.2 Coupling an anharmonic lattice and a linear $F E$ discrete continuum at zero temperature $\ldots \ldots \ldots \ldots$

2.3.3 Coupling an anharmonic lattice and a linear $F E$ discrete continuum at finite temperature $\ldots \ldots \ldots \ldots$. . . . . . . . 41

3 Dynamic Coupled Atomistic and Discrete Dislocation Method 50

3.1 Dynamic CADD Methodology . . . . . . . . . . . . . . 51

$3.1 .1 \quad$ Sub-problem $I I I \ldots \ldots \ldots \ldots \ldots \ldots$

$3.1 .2 \quad$ Sub-problem $I+I I \ldots \ldots \ldots \ldots \ldots \ldots$

3.2 Dislocation Detection and Passing . . . . . . . . . . . . . 59

3.2.1 Dislocation Detection . . . . . . . . . . . . . . 59

3.2 .2 Dislocation Passing . . . . . . . . . . . . . . . . . . . . 64

3.3 Dynamic Solver . . . . . . . . . . . . . . . . . . 65

4 Nanoindenation of ductile crystals $\quad 67$

4.1 Indentation at the nanoscale . . . . . . . . . . . . 68

4.2 Elastic Modulus and Hardness Measurement . . . . . . . . . . . . . 69

4.3 Model System and Simulation Method . . . . . . . . . . . . . 72

4.3.1 Material selection . . . . . . . . . . . . . . 72

4.3.2 Multiscale model description . . . . . . . . . . . . 75

4.4 Verification Simulations . . . . . . . . . . . . . . . . 80

4.5 Results and Discussion . . . . . . . . . . . . . . . . . 85

5 Material Removal Process at the Nanoscale 102

5.1 State of Art of Modeling of Material Removal at the Nanoscale . . . . . 103

5.2 Multiscale Modeling of the Material Removal Process at the Nanoscale . 105

5.2.1 Multiscale Simulations of Nanoscratching . . . . . . . . . 105 
5.3 Multiscale Modeling of Nanocutting . . . . . . . . . . . . 115

6 Conclusions and Future Recommendations 131

6.1 Conclusions . . . . . . . . . . . . . . . 131

6.2 Recommendations for future work . . . . . . . . . . . 135 


\section{List of Figures}

2.1 Schematic illustration of $1 D$ crystal lattice. . . . . . . . . . 13

2.2 Schematic illustration of $1 D$ concurrent coupling of an atomistic chain to an $F E$ discrete continuum. . . . . . . . . . . . . . . . 14

2.3 Schematic illustration of forces in each atom and node. Each atom interacts with its first neighbor in $1 D$ crystal lattice shown in Fig. 2.2 . . . . 15

2.4 Schematic illustration of forces in each atom and node. Each atom interacts with its first and second neighbors in $I D$ crystal lattice shown in Fig.

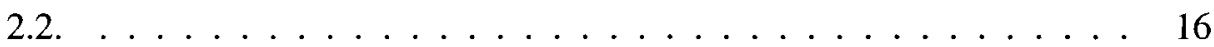

2.5 Left and right hat basis functions $\ldots \ldots \ldots \ldots$

2.6 Wave propagation from atomistic region to continuum region at zero temperature. Potential in atomistic region is harmonic. The disturbance wave-

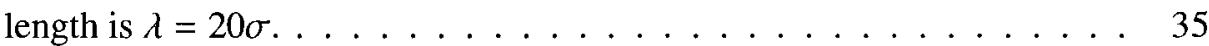

2.7 Wave propagation from atomistic region to continuum region at zero temperature. Potential in atomistic region is harmonic. The disturbance wave-

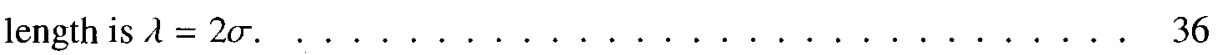

2.8 Dispersion relation for different values of $\Delta x \ldots \ldots \ldots$

2.9 Initial displacement with different wave length. . . . . . . . . . 38

2.10 Wave propagation through the coupled model, $\lambda=20 \sigma \ldots \ldots 39$

2.11 Magnitude of wave reflections versus time. Disturbance wavelength is

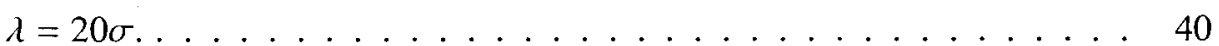

2.12 Magnitude of reflections for different wavelengths. . . . . . . . . . 42 
2.13 Temperature history inside the damping band region during propagation

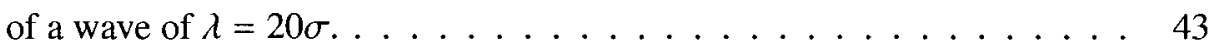

2.14 Wave propagation. $\lambda=20 \sigma$, damping band $=150 \sigma, Q=0.001 . \ldots 44$

2.15 Wave propagation. $\lambda=20 \sigma$, damping band $=150 \sigma, Q=0.1 \ldots \ldots 45$

2.16 Wave propagation. $\lambda=20 \sigma$, damping band $=150 \sigma, Q=10 \ldots \ldots 45$

2.17 Wave propagation. $\lambda=14 \sigma$, damping band $=150 \sigma, Q=0.1 \ldots \ldots 47$

2.18 Wave propagation. $\lambda=14 \sigma$, damping band $=150 \sigma, Q=10 . \ldots 48$

2.19 Wave propagation. $\lambda=30 \sigma$, damping band $=150 \sigma, Q=0.1 \ldots \ldots 48$

2.20 Wave propagation. $\lambda=30 \sigma$, damping band $=150 \sigma, Q=10 \ldots \ldots 49$

2.21 magnitude of reflection versus time for two different thermostating masses, $\lambda=14 \sigma$, dissipating band $=150 \sigma \ldots \ldots \ldots \ldots$

3.1 Schematic illustration of the solution procedure for the CADD method . . 52

3.2 Position of the detection band to detect dislocations Dark grey elements are used in dislocation detection. Filled circles are interface atoms and the open circles are the pad atoms that reside on the finite elements. . . . . . 57

3.3 A series of deformation steps illustrating the non-physical reflection of a dislocation that is not allowed to pass across the atomistic/continuum interface. The mesh is drawn between the atoms in the atomistic region for visualization purposes. The deformed elements clearly highlight the slip plane in the wake of the dislocation. . . . . . . . . . . . 60

3.4 A close-up of one detection element, indicating the three slip planes on which it lies. . . . . . . . . . . . . . . . . 61

3.5 Lagrangian finite strain in triangular detection element [1] . . . . . . . 62

3.6 (a) The dislocation is detected at position $x_{c}$ (b)dislocation is passed across the atomistic/continuum interface at position $\delta+x_{c} \ldots \ldots \ldots 64$

4.1 Typical load-displacement curve . . . . . . . . . . . . . . . 70 
4.2 Baravais lattice vectors of triangular Aluminum $\ldots \ldots \ldots 73$

4.3 Multiscale model geometry for nanoindentation simulation. . . . . . . 75

4.4 A close-up of the atomistic/continuum interface which shows continuum elements, free, pad and damping atoms as well as interface atoms. The circles that lie inside the continuum region are used as a "pad" of atoms to couple the atoms to the continuum region. The grey elements inside the atomistic region are detection elements. . . . . . . . . . .

4.5 Schematic diagram of the repulsive indenter. a) a normal force is applied normal to the surface of the indenter, b) displacement of atoms in contact with the indenter which depends on constant $C$ in Eq. (4.15) $\ldots \ldots$. . 78

4.6 Temperature record inside damping and free atoms regions. . . . . . . . 80

4.7 Displacement distribution of atoms after thermalization process at desired $300 \mathrm{~K}$. The random velocity components of atoms have magnitude corresponding a proper total energy for an equilibrium harmonic system at $300 \mathrm{~K}$ based on the Maxwell-Boltzmann distribution. A damping band of atoms is located between the atom/continuum interface and the undamped atomistic region. Color scale indicates displacement in Angstroms. . . . .

4.8 Average temperature of free atoms zone increases rapidly when the damping band is inactive during loading process. . . . . . . . . 83

4.9 Deformation of substrate during a deep penetration of an indentor. . . . 84

4.10 Load-displacement curves from static and dynamic CADD . . . . . . . 85

4.11 Configuration of positions of the discrete dislocations and the atomic region at maximum load (a), and after unloading (b). . . . . . . 86

4.12 Snapshots of the configuration of the atomistic region during initiation and propagation of dislocations $\ldots \ldots \ldots \ldots \ldots$ 
4.13 Average temperature of damping band and free atoms region during indentation a substrate at four different initial temperatures $100 \mathrm{~K}, 200 \mathrm{~K}$, $300 \mathrm{~K}$ and $400 \mathrm{~K} \ldots \ldots \ldots \ldots \ldots \ldots$

4.14 Initial homogeneous nucleation of dislocation beneath the indenter at three different temperatures, $100 \mathrm{~K}, 200 \mathrm{~K}$ and $300 \mathrm{~K}$, respectively. . . . . . 91

4.15 Variation of critical depth for dislocation nucleation with increasing tem-

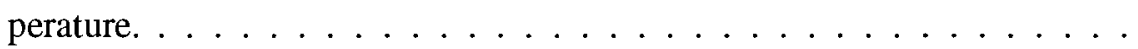

4.16 Nanoindentation load-displacement curves for three different temperatures, $10 \mathrm{~K}, 100 \mathrm{~K}$ and $300 \mathrm{~K}$, respectively. . . . . . . . . . 93

4.17 Initial homogenous nucleation of edge dislocation dipoles at a depth of approximately $0.86 a$, where $a$ is the half width of the contact area between the substrate and indenter, for three different temperature, $10 \mathrm{~K}, 100 \mathrm{~K}$ and $300 \mathrm{~K}$, respectively. . . . . . . . . . . . . . . . . 95

4.18 Maximum critical resolved shear stress prior to dislocation nucleation. The largest values of $\tau_{r}^{\max }$ are not located at the site of nucleation. . . . . .

4.19 Profiles of the atomistic surfaces after $4.2 \mathrm{~nm}$ indentation at two different temperatures, $100 \mathrm{~K}$ and $300 \mathrm{~K}$. The mesh is drawn between the atoms in the atomistic region for visualization purposes and the deformed elements clearly highlight the slip planes in the wake of the dislocations . . . . . 97

4.20 Variation of hardness "load divided by contact area" with increasing indentation depth at $10 K \ldots \ldots \ldots \ldots$

4.21 Variation of hardness at maximum load versus temperature of the atomistic region $\ldots \ldots \ldots \ldots \ldots$

4.22 Load-displacement curves for two different velocities of indentation at 10

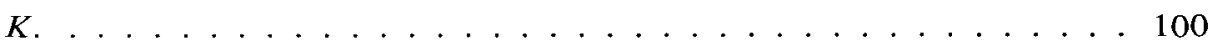

4.23 Local surface plasticity under high speed indentation $400 \mathrm{~m} / \mathrm{s}$. . . . . . 101 
5.1 Multiscale model geometry and a close-up of the atomistic/continuum interface which shows continuum elements, free atoms, pad atoms, damping atoms and interface atoms. The circles that lie inside the continuum region are used as a "pad" of atoms to couple the atoms to the continuum region. The black elements inside the atomistic region are detection elements. . . 106

5.2 Snapshots of plastic deformation and surface finish for two different scratching depth (a) $3.5 \mathrm{~nm}$ (b) $4.1 \mathrm{~nm}$. The tool speed is $100 \mathrm{~m} / \mathrm{s}$. The scratching distance is $100 \mathrm{~nm} \ldots \ldots \ldots \ldots \ldots$. . . . . . . . . . . . . . .

5.3 Close-up of generation and dissociation of dislocations. Scratching depth is $3.5 \mathrm{~nm} \ldots \ldots \ldots \ldots$

5.4 Close-up of generation and dissociation of dislocations. Scratching depth

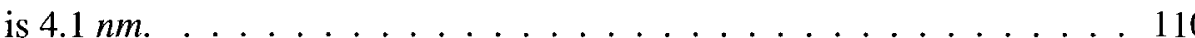

5.5 The variation of normal force of single aluminum crystal for two different scratching depths. . . . . . . . . . . . . . . . 112

5.6 The variation of tangential force of single aluminum crystal for two different scratching depths. . . . . . . . . . . . . . . 113

5.7 Multiscale model geometry and a close-up of the atomistic/continuum interface which shows continuum elements, free atoms, pad atoms, damping

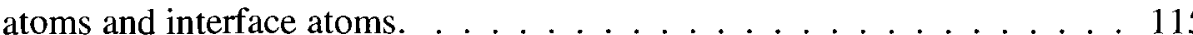

5.8 Simulation plots showing various beginning stages of scratching of an aluminum single crystal. Scratching depth, tool speed and tool radius are $1.7 \mathrm{~nm}, 100 \mathrm{~m} / \mathrm{s}$ and $5 \mathrm{~nm}$, respectively.

5.9 Distribution of dislocations in two different states: (a) compression state, (b) relaxed state. Numbers highlight slip traces in the way of dislocations, the cores of which are at the end of each slip trace. In (a), dislocations 1-3 are below the field of the view, deep in the continuum region. In (b) the relaxed area is located in the right side of the red line. 
5.10 Snapshots of chip deformation and surface finish for two different scratching depths: (a) $1.7 \mathrm{~nm}$,(b) $2.0 \mathrm{~nm}$. Tool speed is $100 \mathrm{~m} / \mathrm{s}$. The scratching distance is $100 \mathrm{~nm} . \ldots \ldots \ldots \ldots$

5.11 The variation of normal force of single aluminum crystal for two different scratching depths, 1.7 and $2.0 \mathrm{~nm}$, respectively. . . . . . . . . . 120

5.12 The variation of tangential force of single aluminum crystal for two different scratching depths, 1.7 and $2.0 \mathrm{~nm}$, respectively. Points $I-I V$ are critical features described in the text. . . . . . . . . . . 121

5.13 Snapshots of generation of dislocations between points I and II on the tangential force curve. . . . . . . . . . . . . 122

5.14 Snapshots of generation of dislocations between points III and IV on the tangential force curve. $\ldots \ldots \ldots \ldots \ldots \ldots$

5.15 Snapshots of chip deformation and surface finish in scratching process for four different tool speed: (a) $200 \mathrm{~m} / \mathrm{s}$; (b) $300 \mathrm{~m} / \mathrm{s}$; (c) $400 \mathrm{~m} / \mathrm{s}$; (d) 800 $\mathrm{m} / \mathrm{s}$. The scratching distance is $100 \mathrm{~nm}$. The depth of scratching is $2.0 \mathrm{~nm} .124$

5.16 Exit-burr formation at final stage of material removal process. The scratching depth is $2.0 \mathrm{~nm}$ and tool moves $100 \mathrm{~m} / \mathrm{s}$. Close-up of exit-burr formation is shown in $(b)$ and $(d) \ldots \ldots \ldots \ldots$

5.17 Close-up of chip profile and surface finish for two different tool speed: (a) $200 \mathrm{~m} / \mathrm{s}$; chip has a crystalline structure, (b) $800 \mathrm{~m} / \mathrm{s}$; chip shows an amorphous structure. The depth of scratching is $2.0 \mathrm{~nm} \ldots \ldots \ldots$. . . . . 126

5.18 The variation of tangential force of single aluminum crystal for four different tool speeds. Scratching depth is $2.0 \mathrm{~nm}$. . . . . . . . . . . . . . 127

5.19 The variation of normal force of single aluminum crystal for four different tool speeds. Scratching depth is $2.0 \mathrm{~nm} . \ldots \ldots \ldots \ldots$ 
5.20 Variation of average tangential and normal forces versus tool speed. The scratching depth is $2.0 \mathrm{~nm}$ and data are collected for $100 \mathrm{~nm}$ plowing distance. . . . . . . . . . . . . . . . . 130 


\section{List of Tables}

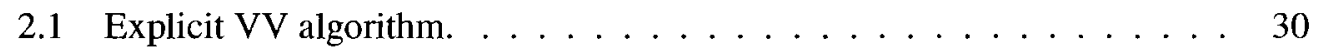

2.2 Explicit concurrent algorithm including Nosé-Hoover thermostat. . . . . . 32

2.3 Concurrent Coupling Force Algorithm . . . . . . . . . . . . . 33

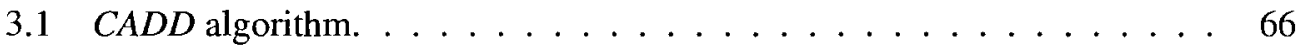

4.1 Calculated in-plane elastic constants for aluminum $\ldots \ldots \ldots$ 


\section{Nomenclature}

\begin{tabular}{|c|c|}
\hline CADD & Coupled atomistic and discrete dislocation method \\
\hline QC & Quasicontinuum method \\
\hline DD & Discrete dislocation method \\
\hline MD & Molecular dynamics method \\
\hline FEM & Finite element method \\
\hline EAM & Embedded atom method \\
\hline $\mathrm{MC}$ & Monte Carlo method \\
\hline DFT & Density-functional theory \\
\hline TBA & Tight-binding approximation \\
\hline CGMD & Coarse-grain molecular dynamics \\
\hline PMMS & Perfectly matched multiscale simulation \\
\hline $\mathrm{LJ}$ & Lennard-Jones potential \\
\hline NEMD & nonequilibrium molecular dynamics \\
\hline$T_{k}$ & Kinetic temperature \\
\hline$T_{\text {ins }}$ & Instantaneous temperature \\
\hline$\beta$ & Friction constant \\
\hline$\omega_{D}$ & Debye frequency \\
\hline$T_{D}$ & Debye temperature \\
\hline$\sigma^{2}$ & Variance \\
\hline$Q$ & Nose'-Hoover thermostat mass \\
\hline
\end{tabular}




\begin{tabular}{|c|c|}
\hline$N_{f}$ & Number of degrees of freedom \\
\hline$\zeta$ & Nose'-Hoover friction constant \\
\hline$\kappa_{B}$ & Boltzmann constant \\
\hline$\hbar$ & Planck's constant \\
\hline$\omega$ & Frequency \\
\hline$\sigma, \epsilon$ & Lennard-Jones parameters \\
\hline B & strain shape function \\
\hline$\epsilon$ & Strain tensor \\
\hline$\sigma$ & Stress tensor \\
\hline $\mathbf{F}_{\text {int }}$ & Internal force vector \\
\hline $\mathrm{P}$ & Indenter load $(n N)$ \\
\hline$\delta$ & Displacement of the indenter $(\mathrm{nm})$ \\
\hline A & Area of contact $\left(\mathrm{nm}^{2}\right)$ \\
\hline $\mathrm{H}$ & Hardness $\left(n N / n m^{2}\right)$ \\
\hline$\Phi$ & Pair potential \\
\hline F & Embedding energy \\
\hline$\rho$ & Electron density \\
\hline$r_{c u t}$ & Cutoff radius \\
\hline$r_{i j}$ & Interatomic distance between atoms \\
\hline $\mathrm{R}$ & Indenter radius \\
\hline$E_{r}$ & Reduced Modulus \\
\hline $\mathrm{E}$ & Young's Modulus \\
\hline
\end{tabular}

xviii 


\begin{tabular}{|c|c|}
\hline$v$ & Poisson's ratio \\
\hline G & Shear Modulus \\
\hline$\lambda, \mu$ & Voigt-average elastic constants \\
\hline $\mathrm{R}$ & Indenter radius \\
\hline b & Burger's vector \\
\hline$F_{c o n}$ & Conservative force vector \\
\hline $\mathrm{F}_{\text {noncon }}$ & Nonconservative force vector \\
\hline $\mathrm{R}_{\text {random }}$ & Random force vector \\
\hline $\mathrm{m}$ & Slip plane normal \\
\hline $\mathrm{u}$ & Displacement \\
\hline$a_{0}$ & Lattice constant \\
\hline$C_{11}, C_{12}, C_{66}$ & Elastic constants \\
\hline$\tilde{u}$ & Infinite elastic continuum displacement field \\
\hline$\hat{u}$ & Displacement field of discrete continuum \\
\hline $\mathbf{M}$ & Mass matrix \\
\hline $\mathbf{K}$ & Stiffness matrix \\
\hline$\tau$ & Resolved shear stress \\
\hline$B$ & Drag coefficient \\
\hline$P_{m}$ & Mean pressure \\
\hline$P_{t}$ & Maximum load during indentation \\
\hline$S$ & Loading stiffness or initial slope \\
\hline $\mathrm{C}$ & Indenter stiffness constant \\
\hline
\end{tabular}

xix 


\section{Chapter 1}

\section{Introduction}

Almost all interesting problems in science and engineering are multi-size-scale and multitime-scale in nature. In the size-scale, materials are made up of atoms and electrons at the atomistic scale, and at the same time are characterized by their natural geometric dimensions which are usually several orders of magnitude larger. Atomistic processes occur at the time scale of nano or femto-seconds, but events at the macroscale are happening over much longer times, at least longer than several seconds. This multiscale nature of problems can be sometimes ignored during modeling and simulation of materials at the macroscale. However, these higher-level models will have limited accuracy for certain types of problems. In some cases, we need to increase the accuracy of macroscale models (higher-level) by including effects from the microscale (lower-level). Consider the example of plastic flow in which we are interested in the flow rate and strain field of the material; the effect of the atomistic or microscale processes are generaly modeled by equations of state and constitutive relations, mostly empirical in nature [2]. Accuracy is particularly an issue for systems below several hundred microns in size, such as nanostructures. In such cases, the constitutive equations might not be valid anymore and the modeling error can be significant.

At the same time, these higher-level models completely neglect microscale mechanisms which are sometimes of interest and only can be captured using lower-level models. Take the example of indentation at the nanoscale. It is often of interest to know 
microstructural details such as the conformation of the dislocations, or grain boundary motion, and correlate these details with the macroscale signature of the indenter on the sample [3]. For such reasons, one might be tempted to choose a fully microscale model that provides proper accuracy, closer to the physical foundations in many cases. However, these models are often computationaly very expensive. Consider for example the simulation of crack propagation in a solid using molecular dynamics $(M D)$ in scales close to several micrometers. Current massively parallel supercomputer simulations can handle about $10^{9}$ atoms using a simple empirical potential, amounting to a volume of less than 1 cubic micron. Even with substantial future increases in computational power, attaining the scale needed to conduct an $M D$ simulation of our example is unlikely. This is where multiscale modeling comes in. By coupling macroscale and microscale models, it is possible to take advantage of both the simplicity and efficiency of the macroscale models, as well as the accuracy of the microscale models. The basic task of multiscale modeling is to design combined higher-level and lower-level computational methods that are much more efficient than solving the full lower-level model, and at the same time gives the information that we need to the obtain desired accuracy.

\subsection{Simulation of Solids at Different Size/Time-scales}

In the context of materials simulations, one can distinguish several characteristic sizescales ranging from the atomic scale $\left(\sim 10^{-9} \mathrm{~m}\right.$ or a few nanometers) to the mesoscopic scale $\left(\sim 10^{-4} \mathrm{~m}\right.$ or hundreds of micrometers $)$, to the macroscopic scale $\left(\sim 10^{-2} \mathrm{~m}\right.$ or centimeters and beyond). Phenomena at each length scale typically have a corresponding timescale that, in correspondence to the different size scales, ranges roughly from femtoseconds to nanoseconds, to milliseconds and beyond.

At each size and time scale, well-known and efficient computational approaches have been established to handle the relevant phenomena. At the atomic scale, quantum chemistry methods [4] can be employed to treat electrons explicitly and accurately. These meth- 
ods are computationally too expensive to handle more than a few tens of electrons. Methods based on density-functional theory (DFT) are somewhat less accurate than quantum chemistry methods, but can be effectively applied to systems containing several hundred atoms within timescales of a few picoseconds. The semi-classical tight-binding approximation (TBA), although typically not as accurate as DFT methods, can extend the reach of simulations to a few nanometers in linear size and a few nanoseconds in timescale for dynamic simulations [5].

At the microscopic level, molecular dynamics $(M D)$ and Monte Carlo $(M C)$ simulations using emperical interatomic potentials are usually performed $[6,7,8]$. Although not as accurate as the $D F T$ and $T B A$ methods, these classical simulations are able to provide insight into atomic processes involving considerably larger systems, reaching up to several million atoms. The timescale that $M D$ simulations can reach is up to a microsecond.

At the mesoscopic scale, the atomic degrees of freedom are not explicitly treated, and only larger-scale entities are modeled. For example, in treating dislocations, recent progress has been concentrated on the so-called dislocation dynamics approach $[9,10]$, which deals with the kinetics of dislocations. The method can be used to study systems up to few tens of microns in size. Finally, for the macroscopic scale in solids, finite element $(F E)$ methods and boundary element $(B E)$ methods are routinely used to examine the large-scale properties of materials considered an elastic continuum $[11,12]$.

\subsection{Classification of Multiscale Methods}

There already exist a few review papers and special editions of articles on multiscale simulation of materials in the literature $[13,14]$. Therefore, a number of classification frameworks has been proposed to categorize these methods. Here, in general, we classify the multiscale simulations into two major families, serial (message-passing or sequential) methods and concurrent (parallel) methods. The serial methodology attempts to piece together a hierarchy of computational approaches in which macroscale models use coarse- 
grained representations with information obtained from more detailed, microscale models [15]. The characteristic of the systems that are suited for a serial approach is that the macroscale variations decouple from the microscale physics, or the large-scale variations appear homogeneous and quasi-static from the small-scale point of view. Serial coupling methods have been largely limited to parameter passing, and as such they are very widely used in applications such as: constructing empirical atomistic potentials from quantum mechanics simulations, assigning hopping rates in kinetic Monte Carlo schemes using data from molecular dynamics, computing transport coefficients using microscopic models, etc.

The second category of multiscale approaches attempt to link methods appropriate at each scale together in a combined model, where the different scales of the system are considered concurrently and communicate through a matching region or hand-shaking area. In a concurrent simulation, the system is often partitioned into domains characterized by different scales and physics. The challenge of the concurrent approach lies at the coupling between the different regions treated by different methods; a successful multiscale model seeks a smooth coupling between these regions.

The majority of multiscale simulations that are currently in use are serial ones, which are effective in systems where the different scales are weakly coupled. For systems whose behavior at each scale depends strongly on what happens at the other scales, concurrent approaches are usually required. The concurrent multiscale approaches are more general in scope than their serial counterpart. In contrast to serial approaches, concurrent simulations are still relatively new and only a few models have been developed to date [14]. These approaches can be used to study many different problems. For example, dislocation nucleation, grain boundary motion, and finally crack propagation can all be modeled individually or all together in one concurrent method framework, where each of those features happens in a different size scale. What is probably most tempting, however, is that a concurrent approach does not require a priori knowledge of the physical quantities or 
processes of interest. Thus, concurrent approaches are particularly useful when exploring problems about which little is known at the atomistic level and about their connection to larger scales, and when discovering new phenomena [16]. Concurrent coupling methods are also preferred when the constitutive relation depends on many variables, and is therefore difficult to determine by precomputing.

From a numerical viewpoint, the two strategies are very much related. The choice between the two is usually made on the grounds of computational cost. For both serial and concurrent coupling methods, the key issue is to design microscopic simulations that give us the needed macroscopic data. In addition, the results of a concurrent simulation can be used to suggest the functional form for the constitutive relation, which can then be used in a serial coupling method. Therefore, in principle, multiscale simulations could be based on a hybrid scheme, using elements from both the serial and the concurrent approaches. We will not discuss this type of approach in detail here, as it involves no new concepts other than the successful combination of elements underlying the other two types of approaches.

\subsection{Concurrent Coupling of Atomistic-Continuum}

In coupled concurrent atomistic/continuum methods [14], the general methodology is to use a fully atomistic description in one region of material and a continuum description in other regions. Therefore, having a matching or interface region between the atomistic and continuum regions is inevitable. The detailed treatment of the material in the interface is a critical aspect of multiscale modeling. At the present time, methodology of defining a proper matching region is fully developed for static calculations at zero temperature [17], but it remains an area of open research at finite temperature.

At finite temperature (i.e. in dynamic systems), proper matching is much more difficult due to two main reasons. The first is the fundamental incompatibility of the non-local atomistic description and the local continuum description. The second is related to the 
coarse representation of the material in the continuum region. Because of those issues, some frequencies of disturbances initiated in atomistic region are often spuriously reflected; from the atomistic/continuum interface as well as from nonuniform $F E$ grids of the continuum region. Similar events have been noted before at the microscale $[18,19,20]$ and in discrete macroscale simulations such as $F E[21,22]$.

Dynamic mismatch along the atomistic/continuum interface was acknowledged by Rudd and Broughton $[23,24,25,26]$ for the first time. They have noted that the continuumbased origins of the finite element method lead to difficulties in a smooth transition of energy from the atomistic to the continuum when dynamic effects are important. They have suggested a framework to formulate the continuum region using a method called coarse-grain molecular dynamics (CGMD). The CGMD equations of motion smoothly match those of $M D$ as the mesh size is reduced to the atomic scale. To reduce reflection of long-lived, short-wavelength elastic waves from the interface back into the molecular dynamics domain, damping is used in the matching region. Their results exhibit excellent phonon spectra and minimal reflection of elastic waves between different regions.

The Green's function method can be used to dynamically couple atomistic and continuum regions $[27,28]$. This approach to minimize boundary wave reflection seems very promising. However, it requires the computation of the exact linear response functions at the interface, which take the form of matrices of size equal to the number of degrees of freedom along the boundary of the atomistic domain. These matrices are computed by molecular dynamics simulations on domains somewhat larger than the one of interest. The method, while highly accurate, is computationally expensive.

E et al. $[29,30]$ have developed an approximate, nonreflecting interface by constructing time integration formulas that eliminate spurious reflections. They have modified the governing equations of motion for the atoms near the interface to reduce phonon reflection from the interface. The method holds promise but has not been fully explored or extended beyond relatively simple test problems. 
Xiao and Belytschko have developed a quasistatic [31] and dynamic [32] bridging method for coupled atomistic/comtinuum problems. In their method, the coupling forces are obtained by imposing a kinematic constraint between the continuum and atomistic domains. For quasistatic problems, they employ either edge-to-edge coupling or kinematic coupling of overlapping segments of the continuum and atomistic domains. They avoid edge-to-edge coupling for dynamic problems, stating that an overlap region is needed to handle components of the atomistic response that cannot be resolved at continuum scales.

Park et al. [33, 34], Wagner et al. [35] have developed a dynamic coupling method called the bridging scale method. The method is valid for finite-temperature problems. In the bridging scale, the continuum representation exists everywhere in the domain, including those area in which the $M D$ is present. The method uses a projection operator to decompose the displacement field into orthogonal coarse (continuum) and fine (atomistic) scales. The approach assumes that the finite element and molecular dynamics solutions exist simultaneously in the whole domain, but the molecular dynamic calculations are performed only in the regions that are necessary. A scaling of the fine and coarse scale potential is used in conjunction with Lagrange multipliers on the overlapping region. The method projects the fine scale solution onto the coarse scale solution in the bridging domain at each time step. Thus, it filters the high frequency components at the interface. Furthermore, since the method is not limited by linearization, there is the possibility of applying the approach to nonlinear problems. This method was examined for several one and two dimensional multiscale examples such as crack and wave propagation [36].

To and Li proposed a new atomistic-continuum multiscale method called the Perfectly Matched Multiscale Simulation (PMMS) method [37, 38]. This method uses a domain decomposition approach similar to the bridging scale method. The method only includes an Absorbing Boundary Condition (ABS) along the interface to reduce spurious reflection. The high frequency waves are absorbed and eliminated during multiscale simulation using a Perfectly Matched Layer (PML) [39] which is appended outside the atomistic region. 
Liu and Li [40] have extended the zero-temperature PMMS to the case of finite temperature. The approach is based on the novel idea of distributed coarse scale thermostats. Instead of viewing the entire coarse scale region as one large bath, they regard each coarse scale node as a thermostat and attach a set of atoms to it. At the atomistic region, the atoms associated with a particular coarse scale node are treated with Nose-Hoover dynamics to conserve temperature.

A finite temperature coupled method was developed by Qu et al. [41]. In this approach, a stadium damping concept [42] is introduced as a method to absorb energetic pulses along the atomistic/continuum interfaces. The approach inserts an additional Langevin damping term into the equations of motion for atoms in a boundary region near the atomistic/continuum interface, with the damping coefficient ramped linearly over the width of the region. The continuum region deformations are computed using static finite element method updated stochastically over time scales comparable to the Debye frequency of the atoms using time-averaged displacements at the atomistic/continuum interface. The method shows promise to equilibrate the inner atomistic region at a desired temperature and to absorb elastic pulses initiated in the atomistic region which moves toward the atomistic/continuum interfaces.

The second issue that has to be taken into account to carry on a dynamic multiscale simulation is the capability of the method to handle inelastic deformation or plastic flow through the entire coupled model. For some applications such as nanoindentation, the multiscale method must work both at the level of the material description (by incorporation of both elastic regions and atomistic regions) and also at the level of defects, in that the dislocations must be treated differently depending on the region in which they reside. A few dynamic multiscale methods such as quasicontinuum [43] and coupled atomistic/discrete dislocation method $[44,45]$ are capable of modeling of plasticity and motion of dislocations or defects during the simulation. These concurrent methods for coupling molecular dynamics models with a continuum are useful for studying local phe- 
nomena such as nanoindentation $[17,44]$ and nanoscratching $[46,47]$. They permit the use of far fewer degrees of freedom than in strict molecular dynamics models, since the resolution in the subdomain modeled by continuum mechanics can be made far coarser without loss of accuracy.

\subsection{Outlook}

There currently is no reliable concurrent atomistic-continuum method capable of coupling continuum strain fields to atomistic dislocation motion and lattice vibrations at finite temperature. In this dissertation, we present a robust multiscale material modeling methodology, in which classical finite temperature $M D$ is coupled with a continuum description. The method uses a linear $F E$ method to model the continuum region. In our model, the continuum subdomain serves primarily as a boundary model that provides low frequency impedance and a sink for the energy associated with outgoing waves of the $M D$ model. The method contains a damping band (thermostating band) to dissipate the spurious energy reflections at the $M D / F E$ interface and at the $F E$ mesh as it coarses away from the interface. We first present and analyse the coupled formulation, with one spatial dimension, employing linear springs and a nonlinear potential, demonstrating its efficacy through numerical examples. We then describe a full $2 D$ implementation.

A unique characteristic of the developed method is its ability to accommodate discrete dislocations in the continuum region for capturing long-range dislocation plasticity. The method thereby further reduces the computational memory and time from what would be required to perform the same simulations using a fully atomistic model. The model is tested for several applications, namely nanoidentation, nanoscratching and nanocutting in $2 D$. In all those applications, dislocation nucleation and propagation are key features that are successesfully captured during the conducted simulations.

This thesis is organized as follows. Chapter 2 is devoted to the $1 D$ concurrent $M D / F E$ model at finite temperature which serve as a test of the model we will later describe in 
$2 D$. Chapter 2 quantifies the challenges involved when coupling atomistic and continuum simulations, and provides the general governing equations for both the atomistic and continuum regions are described. We also discuss the efficiency of our numerical implementation and present sample $1 D$ calculations for different chosen potentials for the atomistic region.

Chapter 3 is mainly focused on the $2 D$ dynamic coupled atomistic and discrete dislocation $(C A D D)$ method at finite temperature. This is the multiscale modeling scheme that has been developed to simulate the problems in this thesis, building on previous work where the static implementation of $C A D D$ was described [1]. A brief review of the theory and solution procedure is discussed first and then the details of our modifications to the existing static $C A D D$ code are presented. In Chapter 4 and 5, simulations were performed to study nanoindentation and the material removal process at the nanoscale. Finally, some concluding remarks based on the results obtained from the simulations and recommendations for future work are presented.

\subsection{Simplifying Assumptions}

Some simplification assumptions have been made that we wish to state them with complete clarity.

1- Material in the continuum region is considered to be elastically anisotropic.

2- The Continuum region is modeled by linear finite elements with the lumped-mass approximation.

3- The method is initially developed in one or two dimensions.

4- The crystal in $2 D$ examples is a triangular lattice.

5- Temperature dependence of the dislocation drag coefficient $B$ is not considered during the simulations.

6- The time stepping in the atomistic and continuum regions are held in lock-step, as short time steps are needed to maintain stability of the atomistic method. We are aware that for 
a long-run simulation, the short time steps can lead to larger accumulation of error (and larger per-timestep error) in the continuum solution. 


\section{Chapter 2}

\section{Basic Concept of Concurrent Multiscale Model at Finite Temperature: 1D Verification}

In this chapter, the $M D$ and elastodynamic $F E$ methods are coupled together in $1 D$. Loading is dynamically applied and an explicit dynamic solver is used to obtain the responses of the coupled system. Similar to the other concurrent methods, a decision must be made about where to employ continuum and atomistic methods in the problem domain. In this study, we want to choose the atomistic region to be large enough to reveal the characteristics of passing waves. The continuum serves primarily as a supporting boundary that provides an energy sink associated with waves (or energy) leaving the atomistic region. Our focus is on characterizing the effectiveness of the atomistic/continuum interface in mitigating spurious wave reflections. Shiari et al. [44] has shown that in the case of continuous pumping of energy during a multiscale simulation, the serious reflection of energy in a multiscale model can cause overheating of the atomistic region. This destroys long-run simulation results.

There are three key ingredients to our dynamic coupled model; the atomistic model, the thermostat and the continuum. In section 2.1, we review the essential features of these parts. Then in section 2.2, we describe the coupled model we will use to test our method in $1 D$. 


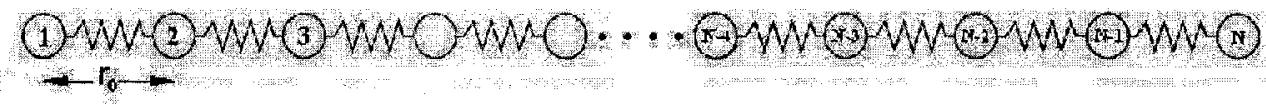

Figure 2.1: Schematic illustration of $1 D$ crystal lattice.

\subsection{General Field Equations of Atomistic and Continuum Regions}

A $1 D$ crystal lattice may be viewed simply as a linear chain of $N$ identical masses (each mass $m$ representing an individual atom) with consecutive masses separated by a distance $r_{0}$ as shown in Fig. 2.1. These masses are attached to each other with spring type connections of length $r_{0}$ that simulate the forces between an atom and its neighbors. The springs are initially unstretched and there are no forces acting on the system so that everything is stationary when all atoms distances are $r_{0}$. In fact, the total energy of the system in this state is zero. In a real crystal lattice, one would never obtain this scenario as the atoms always have thermal energy and are thus in constant motion. We may thus simulate this motion by randomly displacing one or more of the masses from their equilibrium positions. Releasing the masses, the masses would oscillate back and forth in some complicated manner due to the presence of the interconnecting springs.

The model for the $I D M D / F E$ coupled method is shown in Fig. 2.2. The domain is subdivided into two subdomain regions: the atomistic and the continuum region. The overlap of these two domains is denoted by the pad region in the initial configuration. In the pad region, pseudoatoms exist on the continuum side of the interface and overlap physical space with the FEs. Some of the pad atoms coincide with the $F E$ nodes while others may lie within the elements. The pad of pseudoatoms is a device used to help account for the non-local interactions between atoms, so that real atoms at and near the interface see a full complement of neighbors. Without the pad atoms, a non-physical surface energy is introduced into the problem. At this stage of generality, the pad is represented as a set of atoms that are degrees of freedom that can, in principle, be separate 


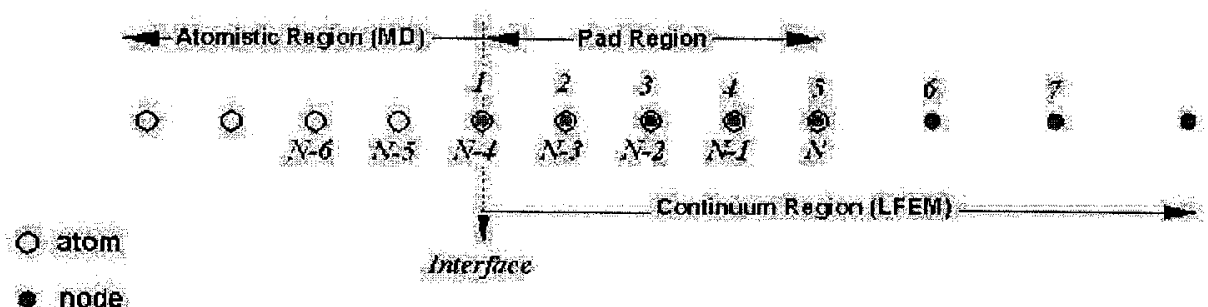

Figure 2.2: Schematic illustration of $1 D$ concurrent coupling of an atomistic chain to an $F E$ discrete continuum.

from the nodes in the $F E$ mesh; it corresponds to the overlap of the two subdomains.

\subsubsection{Molecular Dynamics Method}

In $M D$, successive configurations of a computational cell of $N$ atoms confined to a volume $V$ are computed by solving Newton's equations of classical mechanics. Newton's equations of motion for the $N$ particle system is a set of $N$ coupled second order differential equations in time:

$$
\mathbf{m i r}=\mathbf{f}_{\text {int }}+\mathbf{f}_{\text {ext }}
$$

where $\mathbf{m}$ is a diagonal matrix with atomic masses on the diagonal, $\mathbf{r}$ is position vector, $\mathbf{f}_{\text {int }}$ is internal force vector and $\mathbf{f}_{e x t}$ is external force vector, (") indicates differentiation with respect to time.

For an $M D$ system, the internal force vector can be calculated by differentiating a proper potential energy function $\phi$, which is typically a function of the atomic positions,

$$
\mathbf{f}_{i n t}=-\nabla \phi\left(\mathbf{r}_{1}, \ldots, \mathbf{r}_{N}\right)
$$

Here, for $1 D$ simulation, we use two types of potentials, a simple near-neighbor quadratic (harmonic) potential and Lennard-Jones $(L J)$ potential [48]. The total potential energy of atom $i$ for both potentials can be written as:

$$
\Phi=\sum_{i} \sum_{j \neq i} \phi\left(r_{i j}\right)
$$




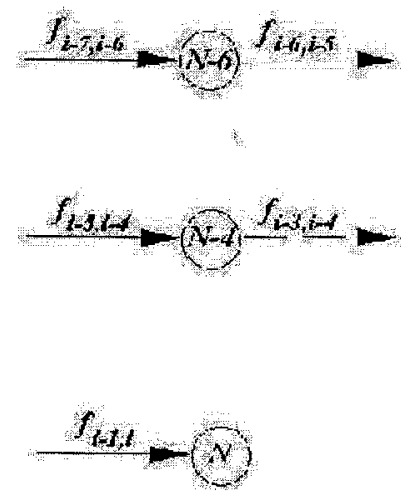

forcesonarom

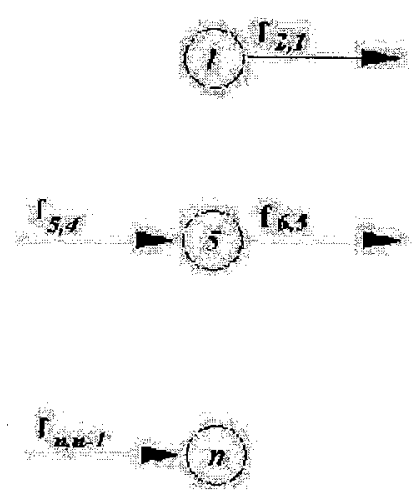

orcen nomes

Figure 2.3: Schematic illustration of forces in each atom and node. Each atom interacts with its first neighbor in $1 D$ crystal lattice shown in Fig. 2.2.

The potential energy function, $\phi$, for the quadratic function is

$$
\phi\left(r_{i j}\right)= \begin{cases}\frac{1}{2} k\left(r_{i j}-r_{0}\right)^{2} & \text { if } \mathrm{j} \text { and } \mathrm{i} \text { are near neighbors } \\ 0 & \text { otherwise }\end{cases}
$$

where $k$ is the stiffness constant, $r_{i j}$ is the distance between atoms $i$ and $j$ and $r_{0}$ is the equilibrium bond length in Fig. 2.1.

The potential energy function, $\phi$ for the $L J$ is expressed as:

$$
\phi\left(r_{i j}\right)=4 \epsilon\left(\frac{\sigma^{12}}{r_{i j}^{12}}-\frac{\sigma^{6}}{r_{i j}^{6}}\right)
$$

where $\epsilon$ and $\sigma$ are constants chosen to fit material properties. The $L J$ model is a pair potential because the energy depends only upon the distance $r_{i j}$ between two atoms.

To save computational time, the $L J$ potential is often truncated at the cut-off distance of $r_{c}=2.5 \sigma$ where

$$
\phi\left(r_{c}\right)=\phi(2.5 \sigma)=4 \epsilon\left(\left(\frac{\sigma}{2.5 \sigma}\right)^{12}-\left(\frac{\sigma}{2.5 \sigma}\right)^{6}\right)=-0.0163 \epsilon=-\frac{1}{61.3} \epsilon
$$

i.e., at $r_{c}=2.5 \sigma$, the $L J$ potential $\phi$ is about $1 / 60 t h$ of its minimum value $\epsilon$ (depth of potential well). Beyond $r_{c}$, the computational potential is set to zero. On the other hand, 

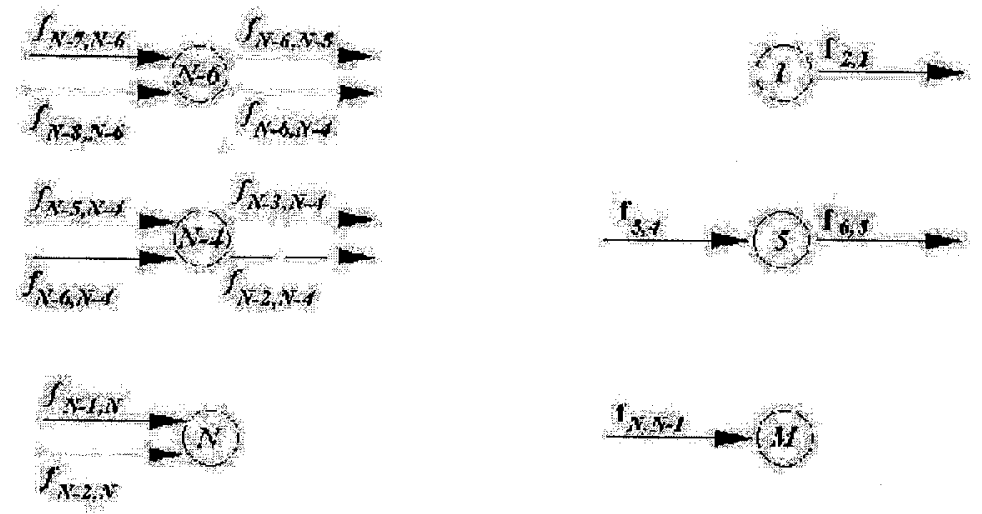

toreesonatoms

forces on nodes:

Figure 2.4: Schematic illustration of forces in each atom and node. Each atom interacts with its first and second neighbors in $7 D$ crystal lattice shown in Fig. 2.2.

to avoid a jump discontinuity at $r_{c}$, as shown in Eq. (2.6), the $L J$ potential is shifted upward a little so that the computational potential would be zero exactly at the cut-off distance $r_{c}$.

For clarity, the computational $L J$ potential is defined as follows:

$$
\phi\left(r_{i j}\right)= \begin{cases}\phi_{L J}(r)-\phi_{L I}\left(r_{c}\right) & \text { for } r \leq r_{c} \\ 0 & \text { for } r>r_{c}\end{cases}
$$

It can be easily verified that $\phi\left(r_{c}\right)=0$, thus eliminating the jump discontinuity at $r=r_{c}$.

It is crucial to note that the $L J$ model is not a realistic potential for most real materials, because of the pair interaction limitation. In accepting this limitation, the $L J$ model is most commonly used in simulations where a general class of effects is studied, instead of specific physical properties, and a physically reasonable yet simple potential energy function is desired.

The total interatomic forces on atom $i$ can be derived as the negative of the gradient of both potential energies in $1 D$ as:

$$
\left.\mathbf{f}_{i}=\sum_{j \neq i} \mathbf{f}_{i j}=\frac{\partial \Phi}{\partial \mathbf{r}_{i}}=\sum_{j \neq i}-k\left(r_{i j}-r_{0}\right) \quad \text { (harmonic }\right)
$$




$$
\mathbf{f}_{i}=\sum_{j \neq i} \mathbf{f}_{i j}=\frac{\partial \Phi}{\partial \mathbf{r}_{i}}=\sum_{j \neq i} 4 \epsilon\left(-12 \frac{\sigma^{12}}{r_{i j}^{13}}+6 \frac{\sigma^{6}}{r_{i j}^{7}}\right) \quad(L J)
$$

where $r_{i j}=r_{i}-r_{j}$.

The equilibrium bond length is the difference in initial positions of two atoms, i.e. $r_{0}=x_{j}-x_{i}$. The interatomic distance can then be written as a function of the initial positions and the displacements $u$ of each atom as $r_{i j}=\left(x_{j}+u_{j}\right)-\left(x_{i}+u_{i}\right)$. Therefore, $r_{i j}-r_{0}=u_{j}-u_{i}=\Delta x$, where $\Delta x$ is the relative displacement between two neighboring atoms.

In our $1 D$ coupling model, we assume that each atom interacts with its first neighbor when we apply a quadratic potential. The equation of motion of atom N-6 in Fig. 2.3 can be derived as a function of the relative displacement:

$$
m \ddot{r}_{N-6}=f_{N-7, N-6}+f_{N-6, N-5}=k\left(u_{N-6}-u_{N-7}\right)-k\left(u_{N-6}-u_{N-5}\right)
$$

The total force on each atom in the $L J$ chain is calculated considering its first- and second-neighbor interactions as shown in Fig. 2.4. For example the equation of motion of atom N-6 is

$$
m \ddot{r}_{N-6}=f_{N-7, N-6}+f_{N-8, N-6}+f_{N-6, N-5}+f_{N-6, N-4}=4 \epsilon \sum_{j=N-8}^{N-4}\left(-12 \frac{\sigma^{12}}{r_{N-6, J}^{13}}+6 \frac{\sigma^{6}}{r_{N-6, j}^{7}}\right)
$$

A useful analogy can be made by comparing the behavior of the harmonic potential to continuum linear elasticity. Note that unlike the $L J$ potential, the harmonic potential cannot recognize bond breaking or separation, because the force is a continuous function of relative displacement. For the $L$ potential, the attractive force dies out quickly after about two interatomic distances, which allows bond breaking if the tensile force is strong enough. In this sense, the harmonic potential is similar to linear elasticity on the atomic level, while $L J$ allows non-linear response.

The mass matrix for the $M D$ section of the $1 D$ coupled model is a diagonal matrix 
with the individual atomic masses on the diagonal. For a $N$ atom system:

$$
\mathbf{m}=\left[\begin{array}{cccccc}
m_{1} & 0 & 0 & \cdots & 0 & 0 \\
0 & m_{2} & 0 & \cdots & 0 & 0 \\
\vdots & & & & & \\
0 & 0 & 0 & \cdots & 0 & m_{n}
\end{array}\right]
$$

where $m_{i}$ are the masses of each individual atom.

\subsubsection{Modeling Issues in Constant Temperature MD}

In this section, we review the basic details of $M D$ thermostats, which are central to our coupled model. We follow closely the reviews by $[49,50,51]$, where the reader may seek further detail.

Based on ensemble theory, for any given macrostate, it is statistically likely that there are a large number of distinct microstates at any given instant $t$. As the real time evolves, the system continually switches between one microstate to another traversing the entire ensemble space given enough time. The averaged material property that we observe is then the result of all the microstates occupied by the system during its evolution process. The collection of all microstates that are all part of the same macrostate is called the ensemble. The celebrated ergodic hypothesis allows us to take time average of an evolving system (as computed by molecular dynamics) and equate it to the spatial average of all the microstates spanned by the ensemble phase space [49].

In near-equilibrium molecular dynamics simulations of steady states, the thermostat is of paramount importance. The system is driven by an external force, i.e., energy is pumped into the system and dissipated into heat. The thermostat is needed to remove this heat, mimicking the role of a heat bath in an experiment. It is, however, possible to combine the driving and thermostating into one simple algorithm.

The goal of this section is to review the methods of $M D$ that can simulate finite temperature processes. In such a simulation, we use a thermostat to maintain the average temperature of the overall system while the local temperature and instantaneous temperature may vary from that desired average. Temperature is a thermodynamic quantity. As 
in a real experiment, the measured temperature of a bath of liquid may vary from the reported value both spatially and temporally. Here the spatial discretization on the order of a domain with a few hundred atoms and times in the range of a few picoseconds is considered. Since $M D$ computes the velocities of individual atoms with time steps of a few femtoseconds, variations in the local instantaneous values of temperatures are actually required to be simulated [52]. Hence there are two basic requirements for an effective thermostat algorithm:

1. The desired temperature (as specified by the input) should be achieved as quickly as possible, in other words the thermostat should be capable of reaching the new isothermal equilibrium condition quickly. The transition time should be minimal.

2. At any given instant of time (after establishing new isothermal conditions) all components of velocity among all the $N$ atoms should follow the Maxwell Boltzmann distribution. For example for $I D$, the speed distribution should satisfy:

$$
f(v)=\frac{4}{\sqrt{\pi}}\left(\frac{m}{2 \kappa_{B} T}\right)^{\frac{3}{2}} v^{2} e^{-m v^{2} / 2 \kappa_{B} T}
$$

where $\kappa_{B}$ is the Boltzmann constant. Eq.(2.13) is a Guassian distribution with mean value of $\langle v\rangle=0$ and standard deviation $\sigma=\sqrt{\frac{{ }_{k} T}{m}}$. It is interesting to note that the average of velocity components remain zero with increase of temperature, while

$$
\left\langle v^{2}\right\rangle=\frac{\kappa_{B} T}{m}
$$

relates the average kinetic energy to the temperature, $\mathrm{T}$.

The thermostat should be capable of achieving Eq.(2.14) in the minimum number of time steps. Numerical experiments carried out show that Eq.(2.14) is not satisfied over a single time step but over a number of time steps:

$$
\frac{1}{N_{s}}\left(\sum_{j=1}^{N_{s}} \frac{1}{N}\left(\sum_{i=1}^{N} v\right)\right)=\left\langle v^{N_{s}}\right\rangle \approx 0
$$

Similarly,

$$
\frac{1}{N_{s}}\left(\sum_{j=1}^{N_{s}} \frac{1}{N}\left(\sum_{i=1}^{N} v^{2}\right)\right)=\left\langle\left(v^{2}\right)^{N_{s}}\right\rangle \approx \frac{\kappa_{B} T}{m}
$$


where $N_{s}$ is the number of time steps.

In a constant temperature $M D$ simulation, the kinetic temperature $T_{k}$, is defined as:

$$
T_{k}=\frac{1}{N} \sum_{i=1}^{N} \frac{m_{i} v_{i}^{2}}{\kappa_{B}}
$$

A naive way to alter the temperature of the system is to change the kinetic energy of the system through increasing or decreasing of the velocity components of each of the atoms. When the velocities are increased for example, this implies that energy is added to the system thus violating the constancy of energy of the constant energy ensemble, unless an equal amount of potential energy is reduced. This reduction however is not automatic since the potential energy is purely a function of atomic positions and the underlying potential energy function. On the other hand, an instantaneous temperature can be defined in $l D$ based on kinetic energy of each atom, $i$, as:

$$
T_{i}^{i n s}=\frac{m v_{i}^{2}}{\kappa_{B}}
$$

The fact that $T_{i}^{\text {ins }}$ is constant does not mean that $T_{k}$ is constant.

Though there are many different thermostats, they can be mainly classified into two types; stochastic and deterministic. In both methods the original equation of motion is modified as:

$$
\mathbf{m} \ddot{\mathbf{r}}=\mathbf{f}_{\text {cons }}+\mathbf{f}_{\text {noncons }}(t)
$$

where $\mathbf{f}_{\text {noncons }}$ is an additional nonconservative force due to the heat bath (or heat sink), and $\mathbf{f}_{c o n s}$ is a conservative force computed via the inter-particle interactions.

In the stochastic methods, a friction force is applied randomly in a way that the velocities satisfy the Boltzmann distribution. The Langevin thermostat, which was proposed by Adelman and Doll [50], is developed from the generalized Brownian motion theory. It models solid lattices at finite temperature using the methods of stochastic theory. In this approach, the molecular system of interest can be thought of as being embedded in a solvent that imposes the desired temperature; the molecules are regarded as solutes. The 
solvent affects the solute through the addition of two terms to the normal Newtonian equation of motion: one is the frictional force and the other is the random force. Then, $\underline{f}_{\text {noncons }}$ in Eq.(2.19) has two components as:

$$
\mathbf{f}_{\text {noncons }}(t)=\mathbf{F}_{\text {friction }}+\mathbf{R}_{\text {random }}
$$

The frictional force takes account of the frictional drag from the solvent as the solute moves. Since friction opposes motion, this force is usually taken to be proportional to the velocity of the particle but of opposite sign.

$$
\mathbf{F}_{\text {friction }}(t)=-\beta \mathbf{v}(t)
$$

The proportionality constant (positive), $\beta$, is called the friction constant. Using the Debye solid model, $\boldsymbol{\beta}$ can be simply expressed as $\boldsymbol{\beta}=\frac{1}{6} \pi \omega_{D}$, where $\omega_{D}$ is the Debye frequency, which is related to the experimentally measurable Debye temperature $T_{D}$ by [50]

$$
\omega_{D}=\frac{\kappa_{B} T_{D}}{\hbar}
$$

where $\hbar$ is Planck's constant.

The random collisions between the solute and the solvent are controlled by the random force, $\mathbf{R}_{\text {random }}(t)$. This force is assumed to have no relation to the particle velocity and position, and is often taken to follow a Gaussian distribution with a zero mean and a variance $\sigma^{2}$ given by [50]

$$
\sigma^{2}=\frac{2 m \beta \kappa_{B} T}{\Delta t}
$$

where $T$ is the desired temperature and $\Delta t$ is the time step.

The frictional force decreases the temperature because $\boldsymbol{\beta}$ is a fixed positive value. The random force is randomly determined from a Gaussian distribution to add kinetic energy to the particle, and its variance is the function of set temperature and time step as Eq. (2.23). Therefore, the random force is balanced with the frictional force and maintains the system temperature at the set value. 
Many thermostats avoid the stochastic force in Eq.(2.19) and use a single friction constant for all atoms. This leads to simplified form

$$
\mathbf{m} \ddot{\mathbf{r}}=\mathbf{F}_{c o n s}-\boldsymbol{\beta} \mathbf{v}(t)
$$

In this case, $\beta$ loses its physical meaning of the friction coefficient and is no longer restricted to positive values. A positive value indicates the heat flows from system to the heat bath. A negative value indicates a heat flow in the opposite direction. The stochastic thermostat is extremely efficient for relaxing a system to the target temperature, but once the system has reached equilibrium, it might be more important to probe a correct canonical ensemble.

The deterministic extended system method was originally introduced by Nosé [53] and subsequently developed by Hoover [54]. The idea is to consider the thermostat as an integral part of the system by addition of an artificial variable $\tilde{s}$ associated with a mass $Q>0$ as well as a velocity $\dot{\tilde{s}}$. The magnitude of $Q$ determines the coupling between the reservoir and the real system, and so influences the temperature fluctuations. The artificial variable $\tilde{s}$ plays the role of a time-scaling parameter, more precisely, the timescale in the extended system is stretched by the factor $\tilde{s}$

$$
d \tilde{t}=\tilde{s} d t
$$

The atomistic coordinates are identical in both systems. This leads to:

$$
\tilde{r}=r \quad \dot{\tilde{r}}=\tilde{s}^{-1} \dot{r} \quad \tilde{s}=s \quad \dot{\tilde{s}}=\tilde{s}^{-1} \dot{s}
$$

The Lagrangian for the extended system is chosen to be

$$
£=\sum \frac{m_{i}}{2} \tilde{s}^{2} \dot{\tilde{r}}_{i}^{2}-\Phi(\tilde{r})+\frac{1}{2} Q \dot{\tilde{s}}^{2}-g \kappa_{B} T_{s e l} \ln \tilde{s}
$$

The first two terms of the Lagrangian represent the kinetic energy minus the potential energy of the real system. The additional terms are the kinetic energy of $\tilde{s}$ and the potential, which is chosen to ensure that the algorithm produces a canonical ensemble where $g$ 
$=$ Number of degrees of freedom in real-time sampling (Nosé-Hoover formalism) and $g=$ Number of degrees of freedom +1 for virtual-time sampling (Nosé-formalism). This leads to the Nosé equations of motion.

$$
\begin{array}{r}
\ddot{\widetilde{r}}_{i}=\frac{\tilde{F}_{i}}{m_{i} \tilde{s}^{2}}-\frac{2 \dot{\tilde{s}} \dot{r}_{i}}{\tilde{s}} \\
\ddot{s}=\frac{1}{Q \tilde{s}}\left(\sum_{i} m_{i} \tilde{s}^{2} \tilde{r}_{i}^{2}-g \kappa_{B} T_{s e t}\right)
\end{array}
$$

These equations sample a microcanonical ensemble in the extended system $(\tilde{r}, \tilde{p}, \tilde{t})$. However, the energy of the real system is not constant. Accompanying the fluctuations of $\tilde{s}$, heat transfers occur between the system and a heat bath, which regulate the system temperature. It can be shown that the equations of motion sample a canonical ensemble in the real system.

The Nosé equations of motion are smooth, deterministic and time-reversible. However, because the time-evolution of the variable $\tilde{s}$ is described by a second-order equation, heat may flow in and out of the system in an oscillatory fashion, leading to nearly periodic temperature fluctuations. The stretched timescale of the Nosé equations is not very intuitive and the sampling of a trajectory at uneven time intervals is rather impractical for the investigation of dynamical properties of a system. However, as shown by Nosé and Hoover [54], the Nosé equations of motion can be reformulated in terms of real system variables. The transformation is achieved through

$$
\begin{array}{lll}
s=\tilde{s} & \dot{s}=\tilde{s} \dot{\tilde{s}} \quad \tilde{s}=s \quad \ddot{s}=\tilde{s}^{2} \ddot{\tilde{s}}+\tilde{s}^{2} \\
r=\tilde{r} & \dot{r}=\tilde{s} \dot{\tilde{r}} \quad \ddot{r}=\tilde{s}^{2} \ddot{\tilde{r}}+\tilde{s}^{2}
\end{array}
$$

and with substituting

$$
\zeta=\frac{\dot{s}}{s}
$$

the Lagrangian equations of motion can be written as

$$
\begin{aligned}
& \ddot{\mathbf{r}}_{i}=\frac{\mathbf{F}_{i}}{m_{i}}-\zeta \dot{\mathbf{r}}_{i} \\
& \dot{\zeta}=\frac{-\kappa_{B} N_{d f}}{Q} T(t)\left(\frac{g}{N_{d f}} \frac{T_{s e t}}{T(t)}-1\right)
\end{aligned}
$$


where $N_{d f}$ is number of degrees of freedom of the system.

In both algorithms, some care must be taken in the choice of the fictitious mass $Q$ and extended system energy $E_{e}$. On the one hand, values of $Q$ that are too large (loose coupling) may cause a poor temperature control. Although any finite (positive) mass is sufficient to guarantee, in principle, the generation of a canonical ensemble, if $Q$ is too large, the canonical distribution will only be obtained after very long simulation times (Nosé-Hoover thermostat with $Q \rightarrow \infty$ is $M D$ which generates a microcanonical ensemble). On the other hand, too small values (tight coupling) may cause high-frequency temperature oscillations. The variable $\tilde{s}$ may oscillate at a very high frequency, it will tend to be off-resonance with the characteristic frequencies of the real system, and it will effectively decouple from the physical degrees of freedom (slow exchange of kinetic energy) [51].

In equilibrium, the variable $\zeta$ is Gaussian with zero mean and variance $\left\langle\zeta^{2}\right\rangle=\frac{\kappa_{B} T}{Q}$. Similarly, from the fluctuations of the kinetic energy one concludes that $\dot{\zeta}$ has vanishing mean and variance $\left\langle\dot{\zeta}^{2}\right\rangle=\frac{2 N_{d f}\left(\kappa_{B} T\right)^{2}}{Q^{2}}$. This results in a typical time scale for the variation of $\zeta$

$$
\tau=\left(\frac{\left\langle\zeta^{2}\right\rangle}{\left\langle\dot{\zeta}^{2}\right\rangle}\right)^{\frac{1}{2}}=\left(\frac{Q}{N_{d f} \kappa_{B} T}\right)^{\frac{1}{2}}
$$

where $\tau$ is the Nosé-Hoover thermostat relaxation time.

This means that the typical $\zeta$ values scale like $N_{d f}^{-\frac{1}{2}}$. In other words, the dynamics becomes more and more Newtonian when the system size is increased, and this means in turn that the method should reproduce canonical ensemble correctly if the system is chosen large enough. On the other hand, this also means that the Nosé-Hoover thermostat does not stabilize the numerical integration of the equations of motion, because it is only based on global feedback [55]. Furthermore, the evaluation of the total kinetic energy involves global communication over all processors if the system is run on a parallel machine with domain decomposition. This is another disadvantage of the Nosé-Hoover thermostat which should not be under estimated [51]. 
If one assumes ergodic dynamic behavior, the Nosé-Hoover thermostat yields a welldefined canonical distribution in both momentum and coordinate space. However, for small systems or for high frequency vibrational modes, the Nosé-Hoover thermostat may fail to generate a canonical distribution [56]. In large molecular systems, the movements of the atoms are sufficiently chaotic that the performance of the Nosé-Hoover thermostat is usually satisfactory [53].

\subsubsection{Finite Element Method}

The continuum side of our coupled model is described by finite elements. If the frequency of external applied load to a structure is higher than roughly one-third of the structure's lowest natural frequency of vibration, then the effects of inertia cannot be neglected and the problem is elastodynamic. In elastodynamic problems the displacement, velocities, strains, stresses and loads are time-dependent. The $F E$ method converts a partial or ordinary differential equation to a discrete, algebraic problem that can be solved numerically.

The procedure involved in deriving the finite element system of equations of a general elastodynamic problem can be stated as follows:

Step 1: Divide the continuum into finite elements. In each discrete element, the displacement field and its two time derivatives are

$$
\begin{array}{r}
\mathbf{d}(x, y, z, t)=\mathbf{N}(x, y, z) \mathbf{u}(t) \\
\dot{\mathbf{d}}(x, y, z, t)=\mathbf{N}(x, y, z) \mathbf{v}(t) \\
\ddot{\mathbf{d}}(x, y, z, t)=\mathbf{N}(x, y, z) \mathbf{a}(t)
\end{array}
$$

where $\mathbf{d}$ is the three component displacement vector, $\mathbf{u}, \mathbf{v}$ and $\mathbf{a}$ are the vector of nodal displacement, velocity and acceleration of an element which are assumed to be a function of time $t . \mathbf{N}$ is the matrix of shape functions, which are typically low order polynomials. For a $1 D$ domain, polynomial elements are defined in terms of a sequence of element endpoints $x_{i}$, where $x_{0} \leq x_{1} \leq \ldots \leq x_{n}$. This sequence of elements is a $1 D$ mesh. A $1 D$ linear element has two nodal displacements, $u_{i}^{e}$ and $u_{i+1}^{e}$, one at each end. For any given set of 

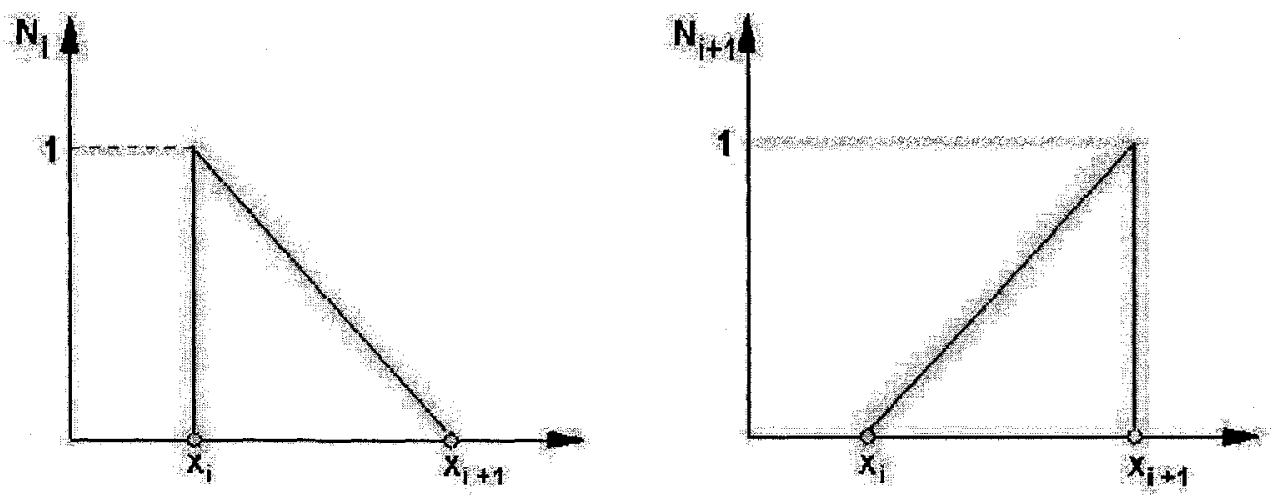

Figure 2.5: Left and right hat basis functions

displacements at the ends, a function is required to convert these into displacements along the length of the element. The obvious selection for the functions is the linear set as two half hat basis functions, one that rises from 0 to 1 across the interval, and one which falls from 1 to 0 (see Fig. 2.5):

$$
\begin{aligned}
N_{i} & = \begin{cases}\frac{x_{i+1}-x}{x_{i+1}-x_{i}} & \text { if } x_{i} \leq x \leq x_{i+1} \\
0 & \text { otherwise }\end{cases} \\
N_{i+1} & = \begin{cases}\frac{x-x_{i}}{x_{i+1}-x_{i}} & \text { if } x_{i} \leq x \leq x_{i+1} \\
0 & \text { otherwise }\end{cases}
\end{aligned}
$$

Step 2: Derive the element characteristic matrices and characteristic load vector. Strains are obtained from displacements by differentiation as $\epsilon=[\partial] \mathbf{u}$. From Eq. (2.33), the stress and strain can be expressed as:

$$
\begin{gathered}
\epsilon=\mathbf{B u} \\
\sigma=\mathbf{D} \epsilon=\mathbf{D B u}
\end{gathered}
$$

where $\epsilon$ is strain vector, $\sigma$ is stress vector, $\mathbf{B}=[\partial] \mathbf{N}$ is strain shape function matrix and $\mathbf{D}$ is elasticity matrix. For $1 D,[\partial]=\left[\frac{\partial}{\partial x}\right]$.

Step 3: Lagrange's equation [57] can be employed to derive the equation of motion of dynamic systems. The final discrete equations can be derived for elastodynamics prob- 
lems:

$$
\mathbf{m u ̈}=\mathbf{f}_{i n t}+\mathbf{f}_{e x t}
$$

The element mass matrix $\mathbf{m}$ can be defined as follows [57]:

$$
\mathbf{m}=\int_{V} \rho \mathbf{N}^{T} \mathbf{N} d V
$$

where $\rho$ is the initial material density, $V^{e}$ is the undeformed volume. The element internal force, $\mathbf{f}_{\text {int }}$, and external load vectors, $\mathbf{f}_{\text {ext }}$, are defined as:

$$
\begin{array}{r}
\mathbf{f}_{\text {int }}=\int_{V} \mathbf{B}^{T} \sigma d V=\mathbf{k u} \\
\mathbf{f}_{\text {ext }}=\int_{V} \mathbf{N}^{T} \mathbf{f}_{b} d V+\int_{V} \mathbf{N}^{T} \mathbf{T} d S+\sum_{i}^{n} \mathbf{p}_{i}
\end{array}
$$

where $\mathbf{f}_{b}$ are body forces, $\mathbf{T}$ are prescribed surface tractions, and $\mathbf{p}_{i}$ are concentrated loads that act at a total of $n$ points on the element. The definition of the stiffness matrix is:

$$
\mathbf{k}=\int_{V} \mathbf{B}^{T} \mathbf{D B} d V
$$

An alternative to performing the integration of Eq. (2.38) is to use a lumped-mass approximation. This involves a simple lumping of the mass of the region at the nodes of the $F E$ model in proportion to the number of the elements in the model. The lumped-mass approach has an advantage in that it generally produces lower frequency estimates and is very easy to calculate. The lumped-mass matrices are diagonal, making computation easier.

The lumped-mass matrix is obtained by simply placing a lumped mass at each node equal to the appropriate proportions of the total mass of the system. If linear shape functions are used, the lumped-mass matrix for a single $1 D$ element can be written as:

$$
[m]=\frac{\rho A l l}{2}\left[\begin{array}{ll}
1 & 0 \\
0 & 1
\end{array}\right]
$$

where $A$ is the initial cross-sectional area of the finite element and $l$ is the length of the element. Eq. (2.42) states that half the mass in the finite element is assigned to each node. 
We use the lumped mass matrix in the following $1 D M D / F E$ coupling example to preserve the diagonal quality of the global mass matrix.

For a linear elastic system, the stiffness matrix of each element takes the familiar form [57]:

$$
\left[k_{i j}\right]=\frac{A E}{l}\left[\begin{array}{cc}
1 & -1 \\
-1 & 1
\end{array}\right]
$$

For the assembled structure, from Eqs. (2.37):

$$
\mathbf{M} \ddot{\mathbf{u}}=\mathbf{F}_{\text {int }}+\mathbf{F}_{\text {ext }}
$$

where

$$
\begin{aligned}
\mathbf{F}_{\text {int }}=\sum_{n=1}^{\text {numel }} \mathbf{f}_{\text {int }} & \mathbf{F}_{\text {ext }}=\sum_{n=1}^{\text {numel }} \mathbf{f}_{\text {ext }} \\
{[\mathbf{K}]=\sum_{n=1}^{n u m e l}[k]_{n} } & {[\mathbf{M}]=\sum_{n=1}^{\text {numel }}[m]_{n} }
\end{aligned}
$$

and numel is the number of elements in the continuum. Summations are written because a typical node, in general, is connected to more than one element. Summations in Eq. (2.45) imply the expansion of element matrices $[\mathbf{k}]$ and $[\mathbf{m}]$ to the structure size so that $\mathbf{u}_{n}$ of each element $n$ becomes identical in size to the structure displacement vector $\mathbf{u}$ in Eq. (2.44).

In this chapter, we assume that no external forces act upon the continuum region, so the total force is equal to the internal force, $\mathbf{F}_{\text {int }}$. Eq. (2.44) is a system of uncoupled, second-order, ordinary differential equations which are discrete functions of space, but still are continuous functions of time.

\subsection{Concurrent Coupling Procedure and the Dynamic Solver}

As discussed in Chapter 1 , in order to effectively combine the regions described by the $M D$ (nonlocal) and $F E$ (local) methods into a single model, one not only has to ensure consistency between the properties of the discrete and continuum media, but also to provide a smooth transition between the two media. In the present work the coupling of the 
two descriptions of the media is brought about by a transition zone in which the $F E$ nodes coincide with the positions of the particles in the $M D$ region, as shown in Fig.2.2. The width of the pad zone is equal to twice the cutoff distance of the interaction potential used in the $M D$ region, four layers of particles in the case of second neighbor $L J$ interactions. This provides a complete set of neighbors within the interaction range for all particles in the $M D$ region. Atoms that belong to the pad zone interact via the interaction potential with the $M D$ region.

The $F E$ and $M D$ methods calculate the physics of the same material in very different ways. One common challenge that we have not discussed yet is the need to use an effective time integration strategy. The time integration scheme is so central to simulating dynamics. Under the assumption of uniform time stepping, a unified mathematical framework for time integration of both $F E$ and $M D$ systems can be considered.

In 1959, Newmark [58] presented a family of single-step integration methods for the solution of structural dynamic problems for both blast and seismic loading. This family of numerical integration methods is based on the direct use of Taylor's series expansion of displacement and velocity fields of systems of ordinary differential equations as Eq. (2.1) and Eq. (2.44):

$$
\begin{array}{r}
\mathbf{u}(t)=\mathbf{u}(t-\Delta t)+\Delta t \dot{\mathbf{u}}(t-\Delta t)+\frac{\Delta t^{2}}{2} \ddot{\mathbf{u}}(t-\Delta t)+\frac{\Delta t^{3}}{6} \dddot{\mathbf{u}}(t-\Delta t)+\ldots \\
\dot{\mathbf{u}}(t)=\dot{\mathbf{u}}(t-\Delta t)+\Delta t \ddot{\mathbf{u}}(t-\Delta t)+\frac{\Delta t^{2}}{2} \dddot{\mathbf{u}}(t-\Delta t)+\ldots
\end{array}
$$

Newmark truncated these equations and expressed them in the following form:

$$
\begin{array}{r}
\mathbf{u}(t)=\mathbf{u}(t-\Delta t)+\Delta t \dot{\mathbf{u}}(t-\Delta t)+\frac{\Delta t^{2}}{2} \ddot{\mathbf{u}}(t-\Delta t)+\beta \Delta t^{3} \dddot{\mathbf{u}}(t-\Delta t) \\
\dot{\mathbf{u}}(t)=\dot{\mathbf{u}}(t-\Delta t)+\Delta t \ddot{\mathbf{u}}(t-\Delta t)+\gamma \Delta t^{2} \dddot{\mathbf{u}}(t-\Delta t)
\end{array}
$$

If the acceleration is assumed to be linear within the time step, the following equation can be written:

$$
\dddot{\mathbf{u}}=\frac{\ddot{\mathbf{u}}(t)-\ddot{\mathbf{u}}(t-\Delta t)}{\Delta t}
$$


Table 2.1: Explicit VV algorithm.

1. Start with position vector $\mathbf{r}$, velocity vector $\mathbf{v}$ and force vector $\mathbf{f}$ at time $t$.

2. Advance the velocity vector to $t+\frac{1}{2} \Delta t$ by integration of the force vector

$\mathbf{v}\left(t+\frac{1}{2} \Delta t\right) \leftarrow \mathbf{v}(t)+\frac{\Delta t}{2} \frac{\mathbf{f}(t)}{m}$

where $\mathrm{m}$ is the mass of a site and $\Delta t$ is the timestep.

3. Advance the position vector by integration of the velocity vector

$\mathbf{r}(t+\Delta t) \leftarrow \mathbf{r}(t)+\Delta t \mathbf{v}\left(t+\frac{1}{2} \Delta t\right)$

4. Recalculation of the force vector at time $t+\Delta t$ is required since the positions have changed

$\mathbf{f}(t) \leftarrow \mathbf{f}(t+\Delta t)$

5.The half-step velocity vector is advanced to a full step using new force vector:

$\mathbf{v}(t+\Delta t) \leftarrow \mathbf{v}\left(t+\frac{1}{2} \Delta t\right)+\frac{\Delta t}{2} \frac{\mathbf{f}(t+\Delta t)}{m}$

The substitution of Eq. (2.50) into Eqs. (2.49) and (2.49) produces Newmark's equations in standard form:

$$
\begin{array}{r}
\mathbf{u}(t)=\mathbf{u}(t-\Delta t)+\Delta t \dot{\mathbf{u}}(t-\Delta t)+\left(\frac{1}{2}-\beta\right) \Delta t^{2} \ddot{\mathbf{u}}(t-\Delta t)+\beta \Delta t^{2} \ddot{\mathbf{u}}(t) \\
\dot{\mathbf{u}}(t)=\dot{\mathbf{u}}(t-\Delta t)+(1-\gamma) \Delta t \ddot{\mathbf{u}}(t-\Delta t)+\gamma \Delta t \ddot{\mathbf{u}}(t)
\end{array}
$$

If $\beta=0$ and $\gamma=0.5$, the Newmark method is the same as Velocity-Verlet (VV) integrator [59] which is the most commonly used time integrator in $M D$. The ubiquity of the $V V$ method (or the central difference Newmark method) is due to several advantages: a) The method is explicit, b) It is simple to implement, c) It has small memory use, and d) It is conditionally stable.

In our method that follows, a lumped diagonal mass matrix is used for the $F E$ region; 
this was motivated by the work of Krieg and Key [60], who showed by numerical studies of simple structures that the lumped-explicit combination is ideal because the discretization errors are compensatory. Explicit integrations by the $V V$ method require that the time step satisfy

$$
\Delta t \leq \frac{2}{\omega}
$$

where $\omega$ is the highest frequency of the mesh; for homogeneous strain in continuum elements this can be expressed as

$$
\Delta t \leq \frac{l}{c}
$$

where $l$ is the minimum element dimension and $\mathrm{c}$ the wave speed. For a $I D$ domain subdivided into elements of unequal length, the highest frequency of the mesh cannot be determined analytically, the highest eigenvalue can numerically be obtained for a series of meshes.

Once space has been discretized by the finite element method and time by the explicit central difference scheme, the dynamic equilibrium of both elastodynamic and $M D$ regions at each time step can be derived as shown in Table 2.1.

In the case of an existing thermostating zone (Nosé-Hoover thermostat) in the $M D$ region, the $V V$ and thermostat algorithm can be simultaneously implemented as shown in Table 2.2.

As shown in Fig. 2.2, consider a $I D$ problem domain consisting of a continuum region and an atomistic region joined by an overlapping region. It will be ideal to satisfy both the displacement compatibility and force equilibrium conditions, in which displacement compatibility is the most important and must be satisfied. This means that

$$
u_{s}^{M D}-u_{s}^{F E}=0
$$

where $u_{s}^{M D}$ and $u_{s}^{F E}$ are displacements at a shared particle $s$ obtained by the $F E$ method and the $M D$ method, respectively, and that

$$
\mathbf{f}_{s}^{M D}+\mathbf{f}_{s}^{F E}=0
$$


Table 2.2: Explicit concurrent algorithm including Nosé-Hoover thermostat.

1. Update thermostat position (or friction constant):

$\zeta\left(t+\frac{1}{2} \Delta t\right) \leftarrow \zeta(t)+\frac{\Delta t}{2} \dot{\zeta}(t)$ using Eq. (2.31)

2. Evolve velocity vector:

$\mathbf{v}(t) \leftarrow \mathbf{v}(t) \exp \left(-\zeta\left(t+\frac{1}{t} \Delta t\right) \frac{\Delta t}{2}\right)$

3. Update velocity vectors ( $V V$ half step update):

$\mathbf{v}\left(t+\frac{1}{2} \Delta t\right) \leftarrow \mathbf{v}(t)+\frac{\Delta t}{2} \frac{\mathbf{f}(t)}{m}$

4. Update position vectors ( $V V$ half step update):

$\mathbf{r}(t+\Delta t) \leftarrow \mathbf{r}(t)+\Delta t \mathbf{v}\left(t+\frac{1}{2} \Delta t\right)$

5. Force vector update:

$\mathbf{f}(t) \leftarrow \mathbf{f}(t+\Delta t)$

6. Update velocity vector ( $V V$ full step update):

$\mathbf{v}(t+\Delta t) \leftarrow \mathbf{v}\left(t+\frac{1}{2} \Delta t\right)+\frac{\Delta t}{2} \frac{\mathbf{f}(t+\Delta t)}{m}$

7. Update thermostat position (or friction constant):

$\zeta(t+\Delta t) \leftarrow \zeta\left(t+\frac{1}{2} \Delta t\right)+\frac{\Delta t}{2} \dot{\zeta}(t+\Delta t)$

8. Evolve velocity vector:

$$
\mathbf{v}(t+\Delta t) \leftarrow \mathbf{v}(t+\Delta t) \exp \left(-\zeta\left(t+\frac{1}{l} \Delta t\right) \frac{\Delta t}{2}\right)
$$

where $\mathbf{f}_{s}^{M D}$ and $\mathbf{f}_{s}^{F E}$ are forces at a overlapping particle $s$ computed in two coupled regions, respectively. The procedure of coupling between atomistic and continuum region is described in Table 3.1. It is essentially what takes place at each execution of step 5 in Table 2.2 . 
Table 2.3: Concurrent Coupling Force Algorithm

1. Atoms in pad region move according to $F E$ node positions to enforce compatibility condition.

2. Force on atoms computed according to Eq. (2.8) or Eq. (2.9) using the pad atoms to correctly embed the atoms in a bulk crystal.

3. Forces on nodes are computed from Eq. (2.39) except node 1 is held fixed to position of atom $N-4$ in Fig. 2.2 which provide displacement boundary condition for $F E$ region.

\subsection{Implementation and Numerical Examples}

To find out how applicable the coupled method presented in this chapter will perform in numerical simulation, we consider now some model problems. Starting with the easiest interatomic interaction, a one dimensional harmonic potential with nearest neighbor interaction, we analyse in section 2.3.1 the concurrent coupling method. We then turn to the more complicated $L J$ potential (with second neighbor interactions and anharmonicity) in section 2.3.2.

\subsubsection{Coupling between a harmonic lattice and a linear FE discrete continuum}

The first set of computational examples correspond to a $1 D$ linear spring-mass atomistic model coupled to a $I D$ linear elastodynamic continuum model. The entire domain is 1400 length units long. There are 701 atoms in the atomistic domain. The continuum domain contains 50 elements. The time step is 0.1 time unit. An overlapping subdomain links the MD and FE domains, as shown in Fig.2.2. We consider nearest-neighbor interactions are present, i.e., only one particle to the immediate left and right of a particle exert a force on that particle as shown in Fig. 2.4. In order to avoid a density (or impedance) mismatch at the boundary, the mass at each node in the transition zone is set equal to the mass of the 
$M D$ particle (meaning that the mesh is refined down to the atomic scale in this region). The thermostat is turn-off in this simulation. This should be a mild test of the coupling method, since both the atomistic and continuum are essentially linear elastic.

We apply initial displacements on the atoms at the left-end portion of the atomistic domain as:

$$
u=A \cos \left(\frac{\pi}{l_{c}} x\right) \quad \text { if } \quad x \leq l_{c}
$$

where $A$, wave amplitude, is 0.005 length unit. We shall set all the initial velocities to zero. Note that Eq. (2.57) is a simple sinusoidal displacement disturbance with a well-defined wavelength $\lambda=2 l_{c}$. The wavelength of the above disturbance can be changed easily by changing $l_{c}$.

Fig. 2.6 shows the wave propagation through both the atomistic and the continuum region. We see that the perturbation travels from left to right of the chain. As the mass densities of the component domains are matched, in the chosen long-wavelength $(\lambda=$ $20 \sigma$ ), there is no reflection at the interface of the two coupled regions. If we decrease the disturbance wavelength 10 times $(\lambda=2 \sigma)$, we see that whenever the distances of the nodes in the $F E$ region change, we get reflections back into the atomistic region as shown in Fig. 2.7. It should be noted that the atomistic wave is dispersive while the continuum wave is not. But in our concurrent model, the $F E$ employed the lumped mass approximation, and from the viewpoint of $M D$ is very similar to a mass-spring system. This phenomenon is well known and has been studied by Bazant [6] and Holmes [22]. This reflection is spurious because in a homogeneous continuum no wave reflection should take place.

The spurious reflection can be explained by considering the different dispersion relations in the system. Each atom inside the chain of 701 atoms with harmonic nearest neighbor interaction has an equation of motion as Eq. (2.10). The resulting dispersion relation is [48]

$$
(\omega(\kappa))^{2}=4 \frac{k}{m} \sin ^{2}\left(\frac{\kappa r_{0}}{2}\right)
$$




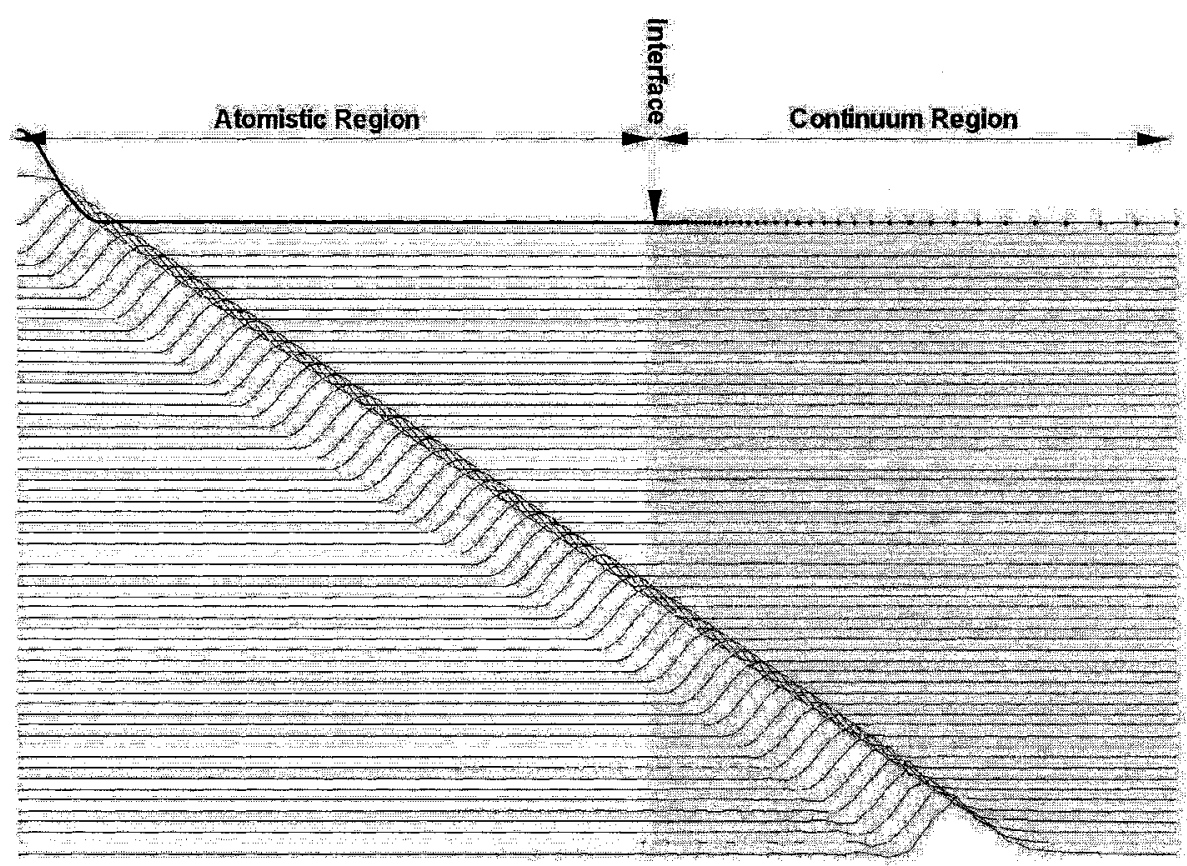

Figure 2.6: Wave propagation from atomistic region to continuum region at zero temperature. Potential in atomistic region is harmonic. The disturbance wavelength is $\lambda=20 \sigma$.

where for a pure sine or cosine wave of frequency $f$ and wavelength $\lambda$ in the chain of atoms, $\omega=2 \pi f$ and $\kappa=\frac{2 \pi}{\lambda}$. The phase velocity is the velocity of each Fourier mode and is given by $C(\kappa)=\frac{\omega(\kappa)}{\kappa}$. In the long-wavelength limit, $r_{0} \leq \lambda$, the wave speed is:

$$
C(0)=\lim _{\kappa \rightarrow 0} \frac{\omega(\kappa)}{\kappa}=\left.\frac{d \omega(\kappa)}{d \kappa}\right|_{\kappa=0}=\sqrt{\frac{k}{m}} r_{0}
$$

which depends only on the parameters $m, k$ and $r_{0}$.

The velocity, $C(\kappa)$, can be normalized by dividing it by the limit velocity, $C(0)$, as

$$
\bar{C}(\kappa)=\frac{\omega(\kappa)}{\kappa} \frac{1}{r_{0}} \sqrt{\frac{m}{k}}=\frac{2}{\kappa r_{0}} \sin \frac{\kappa r_{0}}{2}
$$

which is a function with limit 1 and independent of $k$ and $m$.

In this example, the atomistic and the finite element description for the model problem are in principle the same, only the space discretisation is different. Therefore, we get the same dispersion relation, depending only on the space discretisation. From a harmonic 


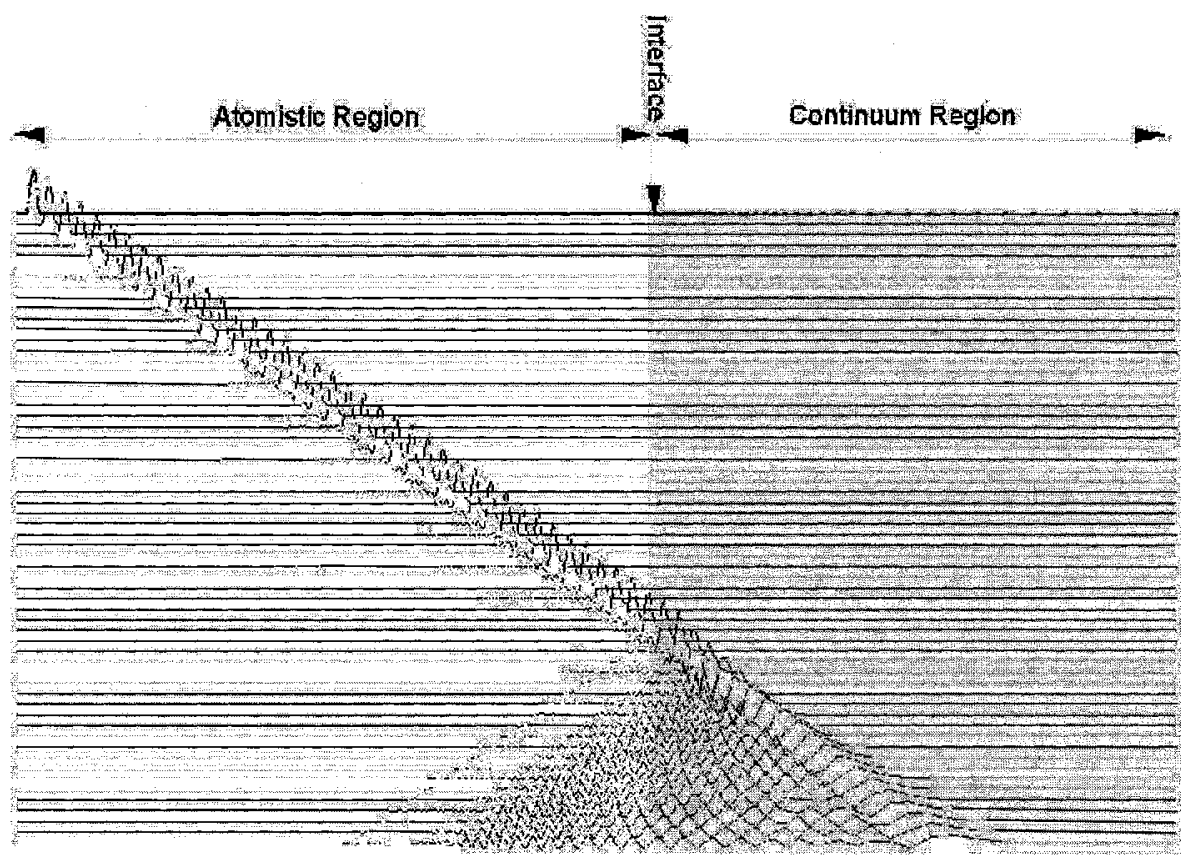

Figure 2.7: Wave propagation from atomistic region to continuum region at zero temperature. Potential in atomistic region is harmonic. The disturbance wavelength is $\lambda=2 \sigma$.

solution, in case of the time discretised equations in the finite element region with node distance $\Delta x[61]$ :

$$
\sin ^{2}\left(\frac{\omega \Delta t}{2}\right)=\frac{(\Delta t)^{2}}{(\Delta x)^{2}} \frac{E}{\rho} \sin ^{2}\left(\frac{\kappa \Delta x}{2}\right)
$$

where $\Delta t$ is time step. $E$ is elasticity constant and $\rho$ is mass density.

The larger the wavelength (smaller wave number), the smaller is the difference in the velocities of the different discretisations as shown in Fig. 2.8 In the limit $\kappa \rightarrow 0$ the velocity is the same for all discretisations. If we have a wave with wave number $\kappa=\frac{\pi}{2 r_{1}}$, it is faster in a simulation with atomic distance $\Delta x=r_{0}$ than in one with $\Delta x=$ $2 r_{0}$. In a simulation with $\Delta x=5 r_{0}$, we cannot even represent this wave, since we need at least two discretisation points per wave length. Therefore, the space discretisation used for the finite elements determines the dispersion relation and the maximum wave vector in the approximate system. We get a different dispersion relation for every different 


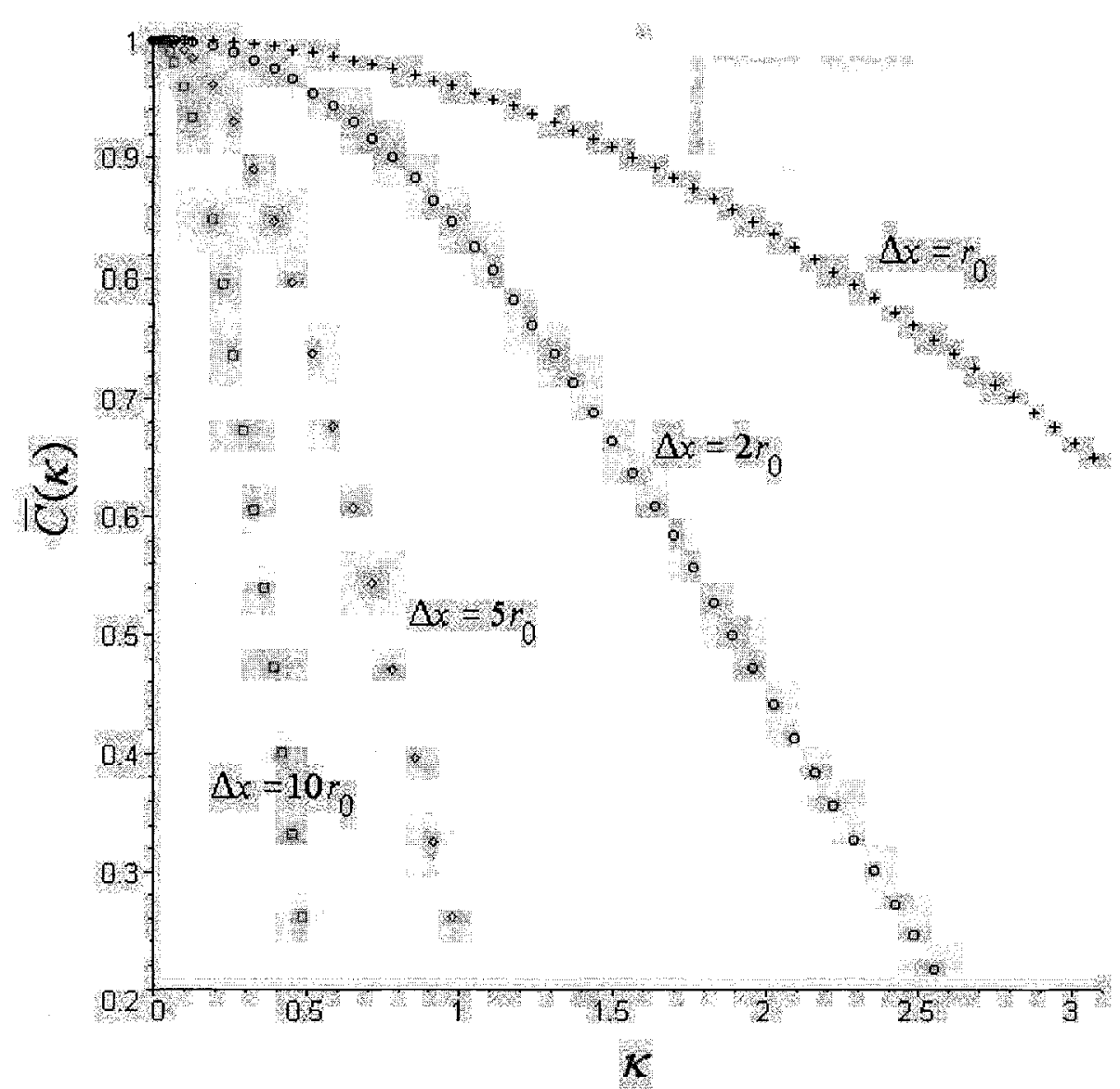

Figure 2.8: Dispersion relation for different values of $\Delta x$.

discretisation, and the velocity of the perturbation changes everywhere the discretisation changes.

If we consider the Taylor expansion of the dispersion of Eq. (2.61) around $\kappa=0$

$$
\omega^{2}=\frac{E}{\rho}\left(\kappa^{2}-\frac{\kappa^{4} \Delta x^{2}}{12}+\frac{\kappa^{6} \Delta x^{4}}{360}+\underline{Q}\left(\kappa^{8}\right)\right)
$$

The above equation can be compared with the atomistic dispersion relation

$$
\omega^{2}=4 \frac{k}{m} \sin ^{2}\left(\frac{\kappa r_{0}}{2}\right)=\frac{k r_{0}}{m}\left(\kappa^{2}-\frac{\kappa^{4} r_{0}^{2}}{12}+\frac{\kappa^{6} r_{0}^{4}}{360}+\underline{Q}\left(\kappa^{8}\right)\right)
$$

As it can be seen, an appropriate selection of the $E$ and $\rho$ in Eq. (2.62) is necessary to have the same velocity in the limit $\lambda \rightarrow \infty\left(\rho=\frac{m}{r_{0}}, E=k\right)$. Therefore, the problem of 


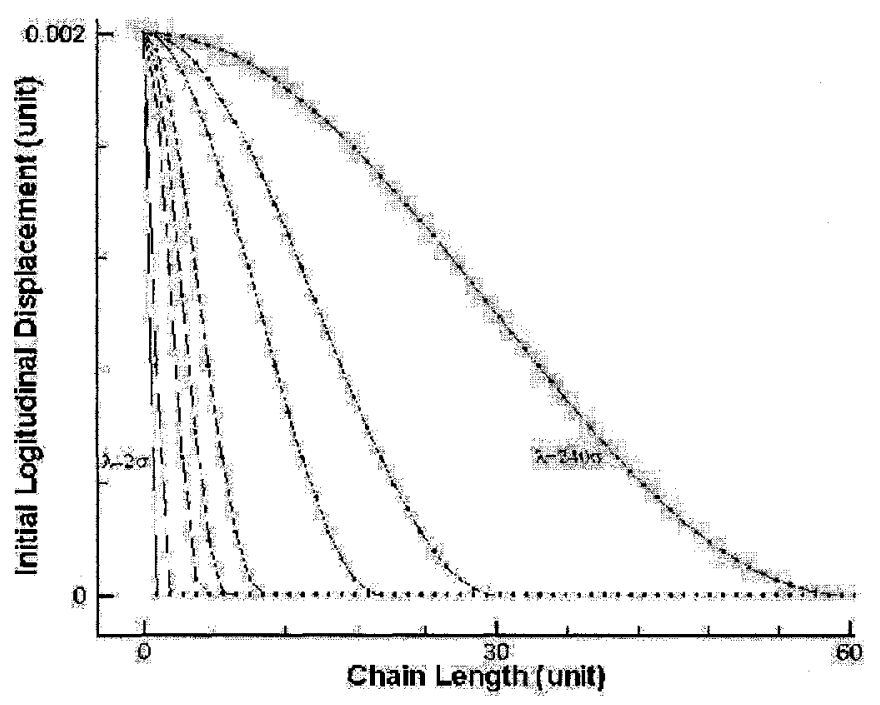

Figure 2.9: Initial displacement with different wave length.

dispersion relation in the finite element region and as a consequence also the reflection of small wavelength, cannot be solved by choosing better parameters, since we have no free parameters left.

In many cases, the difference between the dispersive and non-dispersive solutions is small, and, as can be seen in Fig. 2.6, the coupling between the atomistic and continuum regions shows no dynamic mismatch along the interface for long-wavelength disturbances.

\subsubsection{Coupling an anharmonic lattice and a linear $F E$ discrete con- tinuum at zero temperature}

In this $I D$ example, we use the $L J$ interatomic potential. Here, reduced units are applied, so the results can be transferred to different systems with straightforward scaling. A natural length unit $\sigma$ in Eq. (2.5) and $\epsilon$ is a natural energy unit. The other units are:

$$
t^{*}=t /\left[\left(m \sigma^{2}\right) / \epsilon\right]^{\frac{1}{2}} \quad T^{*}=\kappa_{B} T / \epsilon \quad \rho^{*}=\rho \sigma^{3}
$$

The atomistic region of the model consists of 701 atoms, of which four are located in the pad region as shown in Fig. 2.2. The continuum part has 51 nodes. The coarsening 


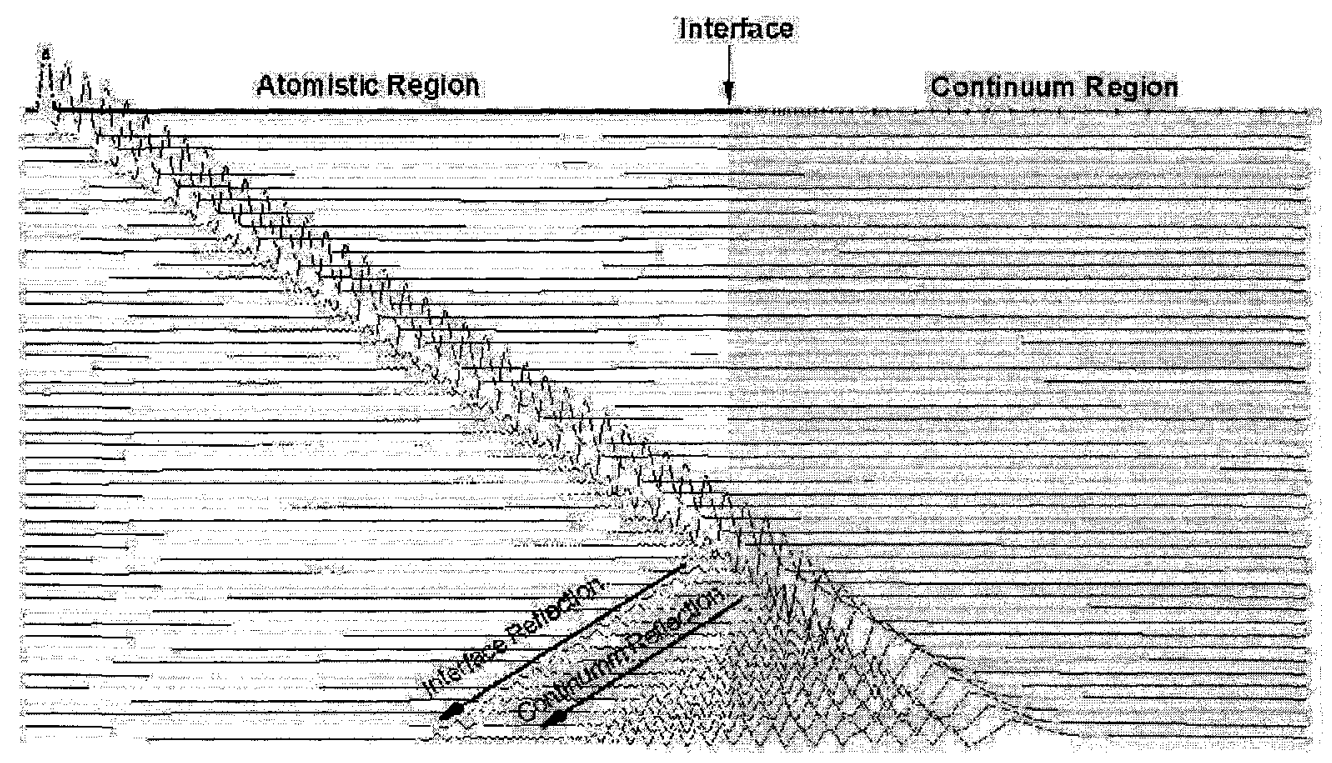

Figure 2.10: Wave propagation through the coupled model, $\lambda=20 \sigma$

ratio for the elements is 1.08 (the length of an element is 1.08 times the length of the element on its left.). Hence, the last element in the model is almost 48 times larger than the first element. The total chain length is 1426 units. Different waves with different wavelengths are applied as initial conditions on the atoms at the left-end section of the atomistic region as shown in Fig. 2.9. The thermostat is turned off in this simulation. The smallest wavelength is $240 \sigma$ and the largest is $2 \sigma$.

Fig. 2.10 shows the wave propagation through the entire model for a wavelength of $20 \sigma$. As it can be seen, there are two types of spurious reflections. The first nonphysical reflection happens at the atomistic/continuum interface. The source of this reflection is the nonlocal/local interaction along the interface which couldn't be seen in Fig. 2.7. Fig. 2.10 also reveals that the perturbation profile which travels from left to right toward the 


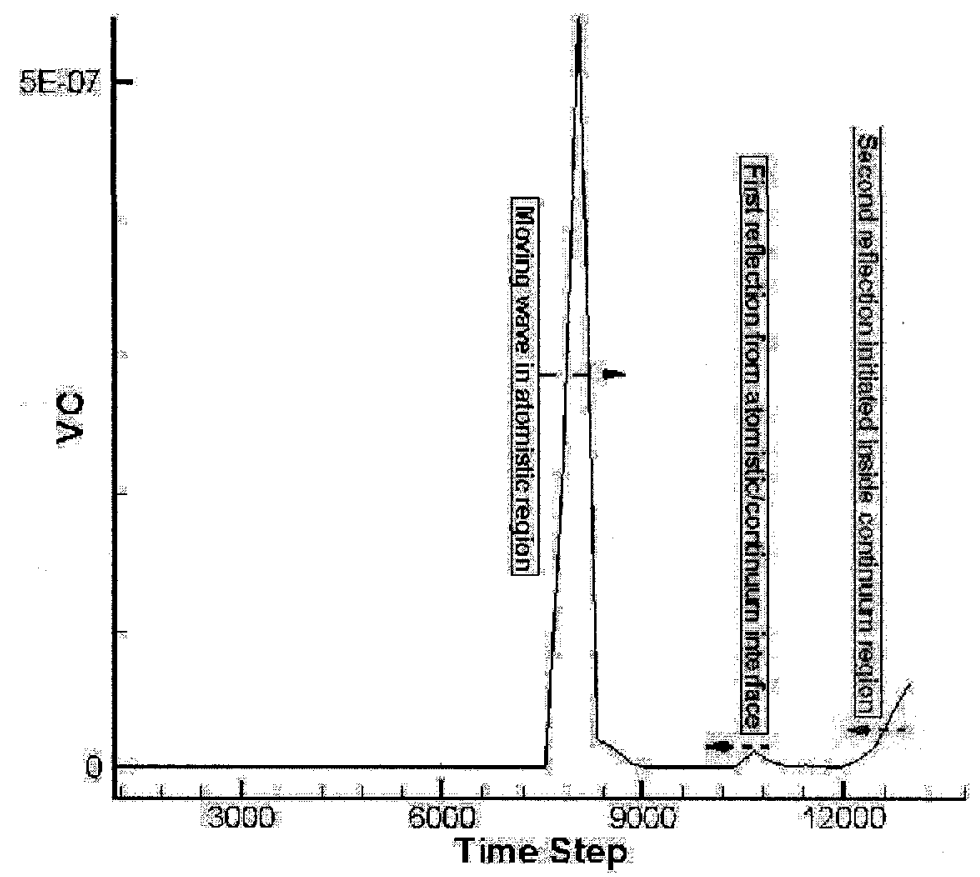

Figure 2.11: Magnitude of wave reflections versus time. Disturbance wavelength is $\lambda=$ $20 \sigma$.

$M D / F E$ interface gradually changes due to dispersion. This phonon dispersion is a real difference in the physical models that cannot be avoided. Fortunately this is typically a small effect except at the short wavelength limit.

The second type of the reflection originates in the discrete continuum region. that is discussed in previous section. The dispersion in the continuum region is also problematic, as this is a non-physical effect that diverges from the pure continuum solution as the element size increases. As it can be seen in Fig. 2.10, waves reflect off the $F E$ mesh as it coarsens away from the atomistic region. This is strongly dependent on the FE mesh and will cause non-uniform reflections. As it can be seen in Fig. 2.10, as the wave arrives from the finite elements of smaller size to the larger one, part of the wave is reflected. The amplitude of the transmitted wave is larger than that of the reflected wave. The 
spurious wave reflection at boundaries of finite elements of different sizes is significant for wavelengths $\lambda$ that are not much greater than the size of the larger finite element [6]. Furthermore, at short wavelength the spurious wave reflection is significant even for very small differences in the size of adjacent finite elements.

To quantify the magnitude of reflections at the atomistic/continuum interface, the mean square velocities of a reference atom, which is located 200 units away from the atomistic/continuum interface, and its two nearest neighbors is calculated. As it is shown in Fig. 2.11, the first reflected energy wave moves back to the atomistic region. The next reflection of energy takes place in the discrete continuum region and this amount of reflected energy is much larger than the reflected energy from the $M D / F E$ interface. As discussed before, the shorter wavelength, high-frequency vibrations cannot be supported by the larger mesh spacings inherent to the outer part of the $F E$ region and will be scattered back into the $M D$ region.

Fig. 2.12 shows the magnitude of reflection $\left(V C_{(\text {First reflected wave })} / V C_{(\text {moving wave })}\right.$ in Fig. 2.11 ) at the interface for different initially applied wavelengths. The long-wavelength phonons propagate with minimal back scattering through the $M D / F E$ interface. Wavelengths larger than $20 \sigma$ do not make a noticeable reflection at all at the interface. It shows that multiscale simulations with low rate loading (low frequency inputs) are almost immune to the overheating issue.

\subsubsection{Coupling an anharmonic lattice and a linear $F E$ discrete con- tinuum at finite temperature}

In this section, we test the effect of a damping band (or thermostating band) on reducing the spurious reflections in our previous test example. A thermostating zone is implemented between the atomistic and continuum region, along the interface of the two regions but inside the atomistic region. The damping band width is considered $150 \sigma$. The temperatures of the atomistic region and damping band are both initialized to 0.1 , after which only the temperature of the band is actively controlled with a Nose-Hoover ther- 


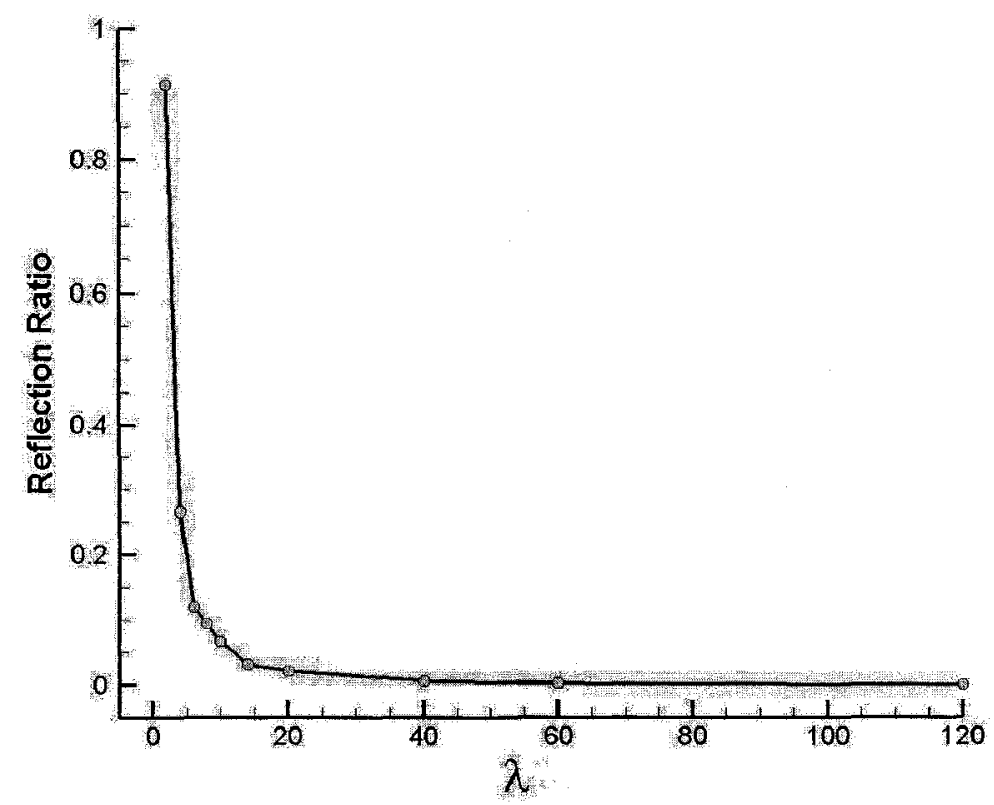

Figure 2.12: Magnitude of reflections for different wavelengths.

mostat (set to 0.1). We apply initial displacements on the atoms at the left-end portion of the atomistic domain as Eq.(2.57) after a warm-up period. The result for the temperature history inside the damping band region during the propagation a wave of $\lambda=20 \sigma$ is presented in Fig. 2.13. As the wave passes through the damping band, the thermostat has the duty of dissipating the added heat to the region and keeps the temperature constant during the simulation. However, as it can be seen in Fig. 2.13, the thermostat experiences a hard time during energy dissipation due to each wave passing event. As it has been mentioned in $\operatorname{Sec} 2.2$, the choice of the thermostat mass, $Q$, or damping frequency, $\frac{1}{\tau}$, is crucial to the effectiveness of the damping band. When $Q$ is too small the Nosé variable can become an isolated mode, it can oscillate independently of the simulated system, and the distribution of the total kinetic energy driven by the oscillator will deviate significantly from the Gaussian distribution. When $Q$ is too large the situation is similar to that in the microcanonical ensemble because the exchange of heat is slow. It has been generally accepted that the 


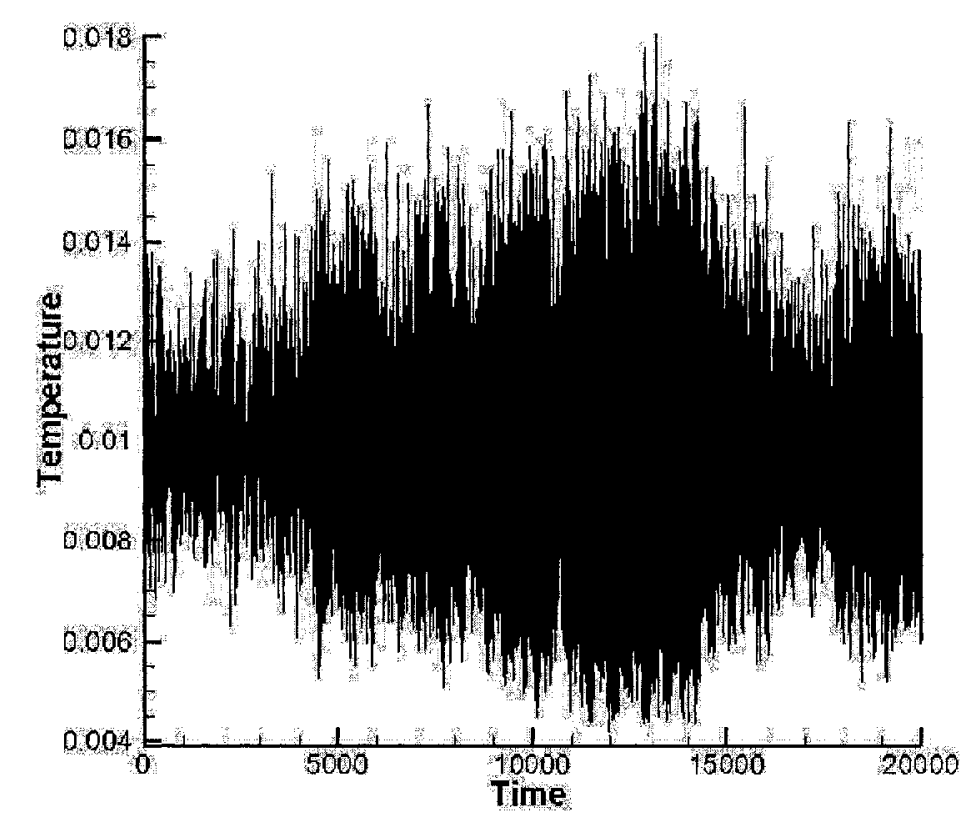

Figure 2.13: Temperature history inside the damping band region during propagation of a wave of $\lambda=20 \sigma$.

correct choice of $Q$ occurs when the self-oscillation frequency of the thermostating variable coincides with some frequency within the original system. As stated previously, for complex systems, where several frequencies exist, the correct method of choosing $Q$ is less clear and is dependent on the coupling between sub-systems and modes. To find a proper value for $Q$, we may start with some recommended values in Ref. [44] and run several trial simulations. Figs 2.14, 2.15 and 2.16 show the results of simulations for three trial values of $Q, 0.001,0.1$, and 10 , respectively.

For small value of thermostat mass, $Q=0.001$, the atomistic region has a tight coupling to the damping band in which atoms experience a high-frequency temperature oscillation. As it can be seen in Fig. 2.14, a major energy reflection happens at the atomistic/damping band interface. If we increase the value of the thermostat mass about ten times, the amount of the reflection at the atomistic/damping band interface decreases, but there is another 


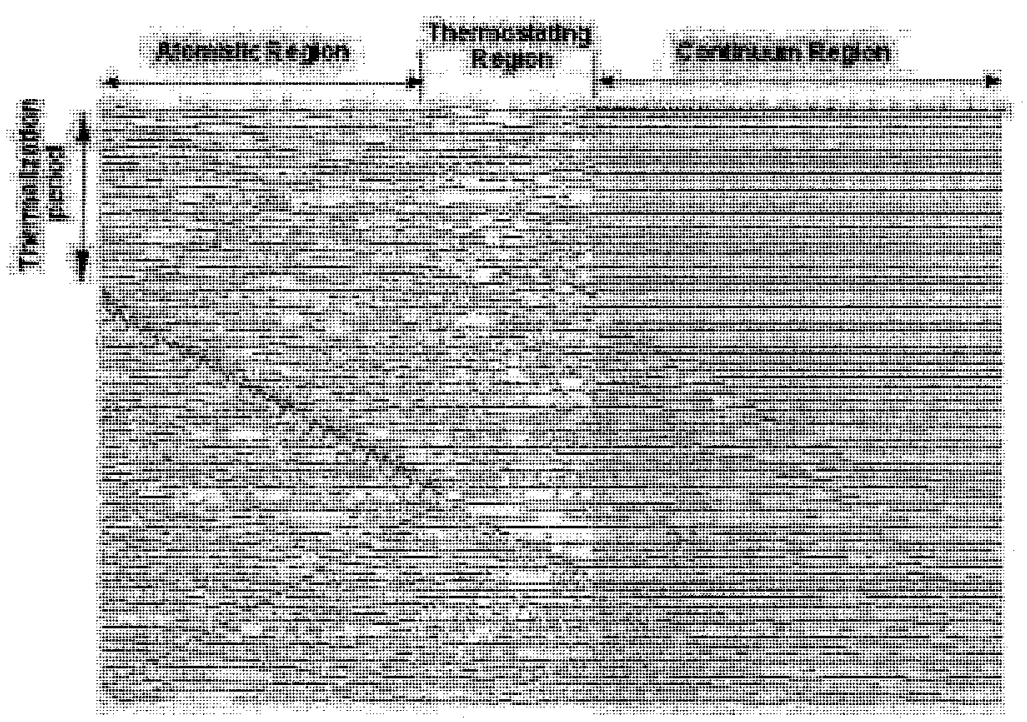

Figure 2.14: Wave propagation. $\lambda=20 \sigma$, damping band $=150 \sigma, Q=0.001$.

energy reflection at the atomistic/continuum interface.

For $Q=0.1$, the wave enters the damping band with no reflection. The wave loses most of its energy inside the band, hence there is no reflection at the atomistic/continuum interface as shown in Fig. 2.15.

The system is tested for a large value of thermostating mass, $Q=10$. In this case, there is a loose coupling between atomistic and damping band regions. In other words, the damping band fails in controlling the temperature or in absorbing the reflected energy. In this case, the presence of the damping band can be ignored during the simulation. Hence, the simulation results are affected by two types of spurious (or nonphysical) reflections, one at the atomistic/continuumm interface, and another originated inside the discrete continuum as shown in Fig.2.16.

To further examine the damping band capability of absorbing the spurious reflection of different traveling wave frequencies, two simulations are performed. In the first simu- 


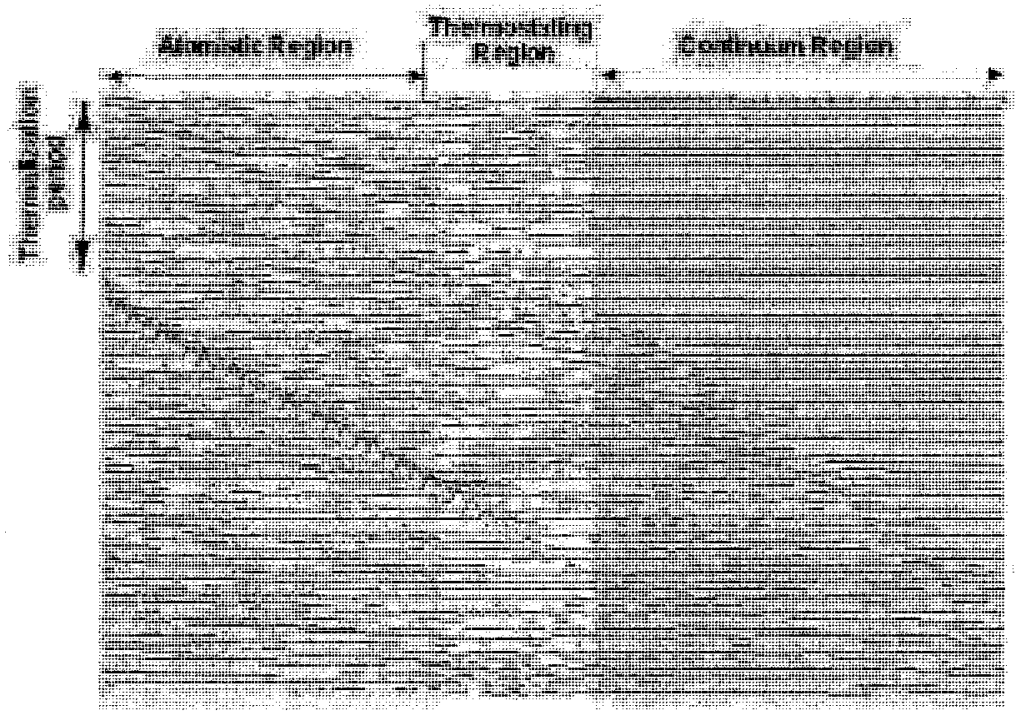

Figure 2.15: Wave propagation. $\lambda=20 \sigma$, damping band $=150 \sigma, Q=0.1$.

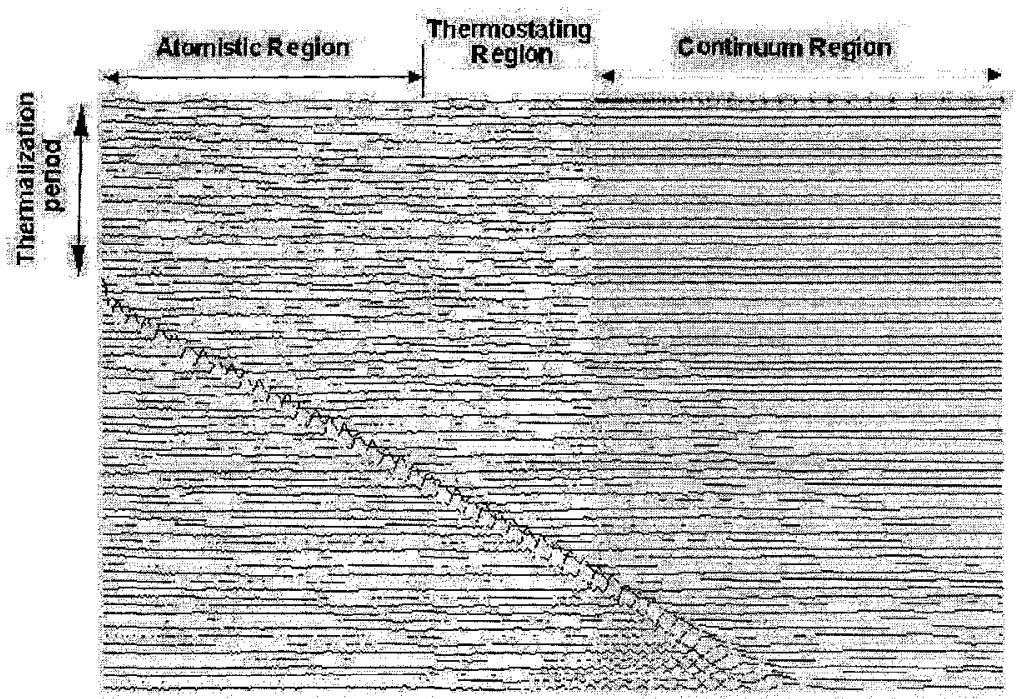

Figure 2.16: Wave propagation. $\lambda=20 \sigma$, damping band $=150 \sigma, Q=10$. 
lation, the wavelength is chosen to be $\lambda=14 \sigma$ which is smaller than the previously tested wavelength, $\lambda=20 \sigma$. As it has been illustrated in Fig. 2.12, this wavelength shows considerable spurious reflection at the atomistic/continuum interface. Fig. 2.17 indicates that the damping band can effectively dissipate the traveling wave energy and all the spurious reflection at the atomistic/continiuum interface. The second group of spurious reflections which are initiated in the discrete continuum region is also damped as it passes across the thermostating zone. Fig.2.18 shows that the reflections of the passing wave at the atomistic/continuum interface and inside the discrete continuum when the coupling between the atomistic and damping regions is loose.

A second simulation is performed using a testing wavelength of $\lambda=30 \sigma$. As can be seen in Fig. 2.12, there is no noticable spurious wave reflection at the atomistic/continuum interface for this wavelength, but there are some energy reflections from the discrete continuum region. Fig. 2.20 shows clearly the second type reflections when the damping band has a loose coupling $(Q=10)$ with the atomistic region. Choosing $Q=0.1$ does not change the passing wave profile in the damping band, but it clearly increases the capability of the damping band to absorb the reflected energies from the discrete continuum region as shown in Fig. 2.20 .

The mean square velocity is used to show the effectiveness of the damping band in absorbing the reflected energies form atomistic/continuum interface and the discrete continuum region. As it can be seen from Fig. 2.21, almost all the reflections are removed by a well tuned damping band $(Q=0.1)$, as compared with an ill-tuned damping band $(Q=10)$.

The presented $1 D$ coupled simulations in this section show that the damping band approach has a significant role on mitigating the reflected waves from the atomistic/continuum boundary and preventing the atomistic region from overheating by a correct choice of $Q$.

In some applications in $2 D$ or $3 D$, such as nanoindentation, nanoscratching and nanomachining, one observes one-way wave flow (or energy pumping) from inside the atomistic 


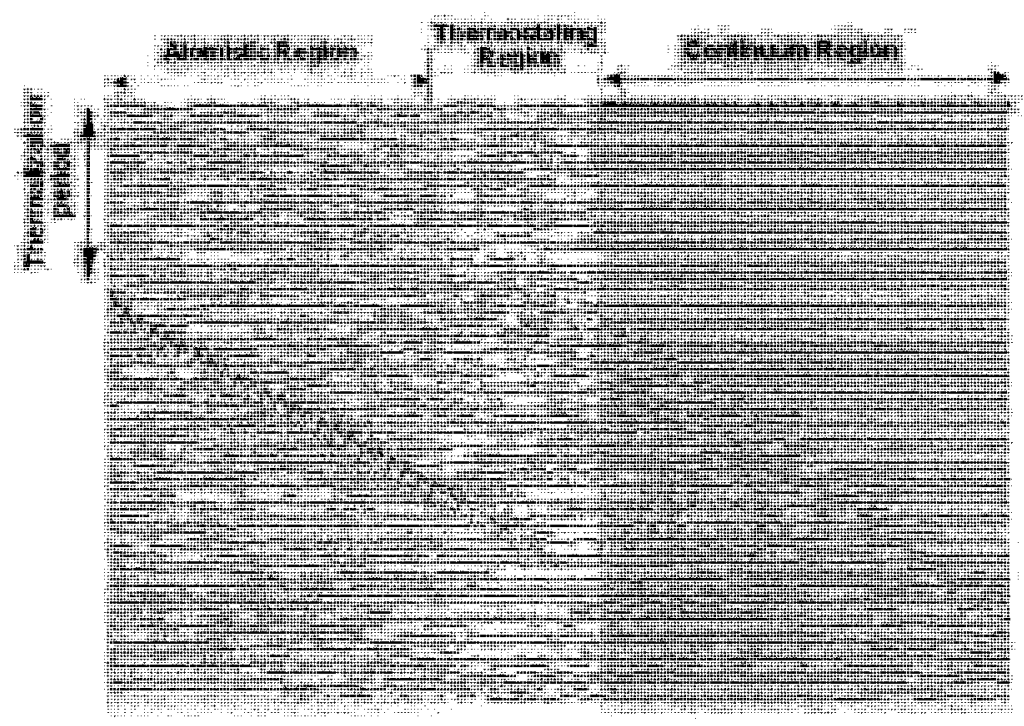

Figure 2.17: Wave propagation. $\lambda=14 \sigma$, damping band $=150 \sigma, Q=0.1$.

region of interest [44]. Distant boundaries of a large the continuum region then behave passively and usually remain stationary. In those cases, we can ignore with a good degree of accuracy the exact solving of the dynamical mismatch problem along the atomistic/continuum interface. We will show in next chapter that the damping band implemented within a $2 D$ coupled atomistic/continuum model can be used as a thermal reservoir to maintain temperature of the atomistic region. At the same time, it also serves to adequately mitigate the energy reflection and overheating of the atomistic region for moderate loading rates like those studied in this thesis. 


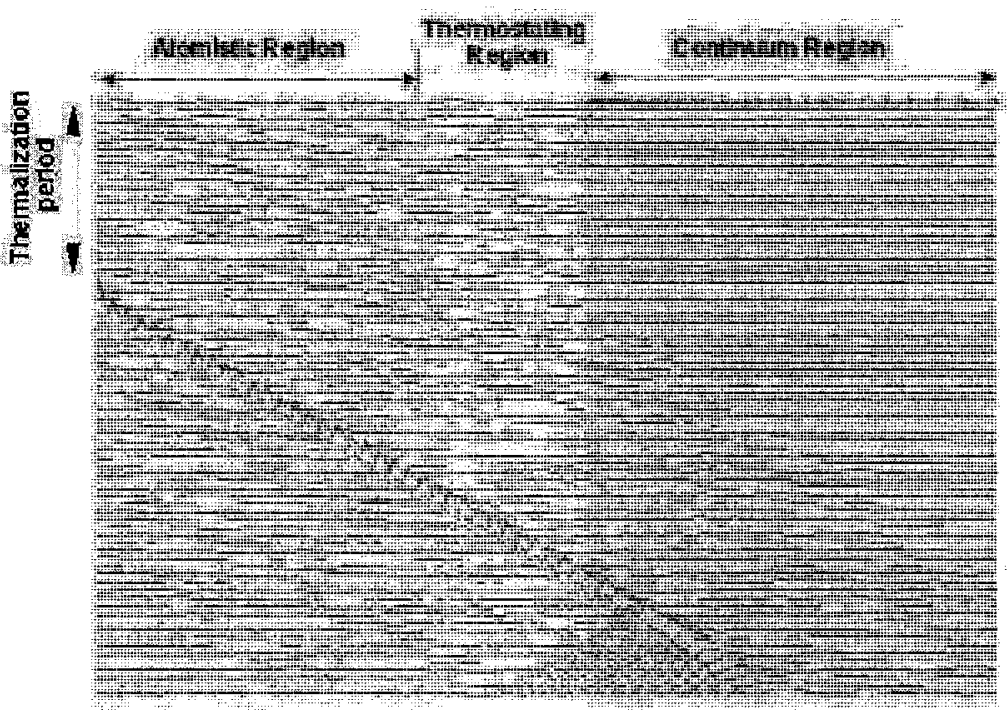

Figure 2.18: Wave propagation $\lambda=14 \sigma$, damping band $=150 \sigma, Q=10$.

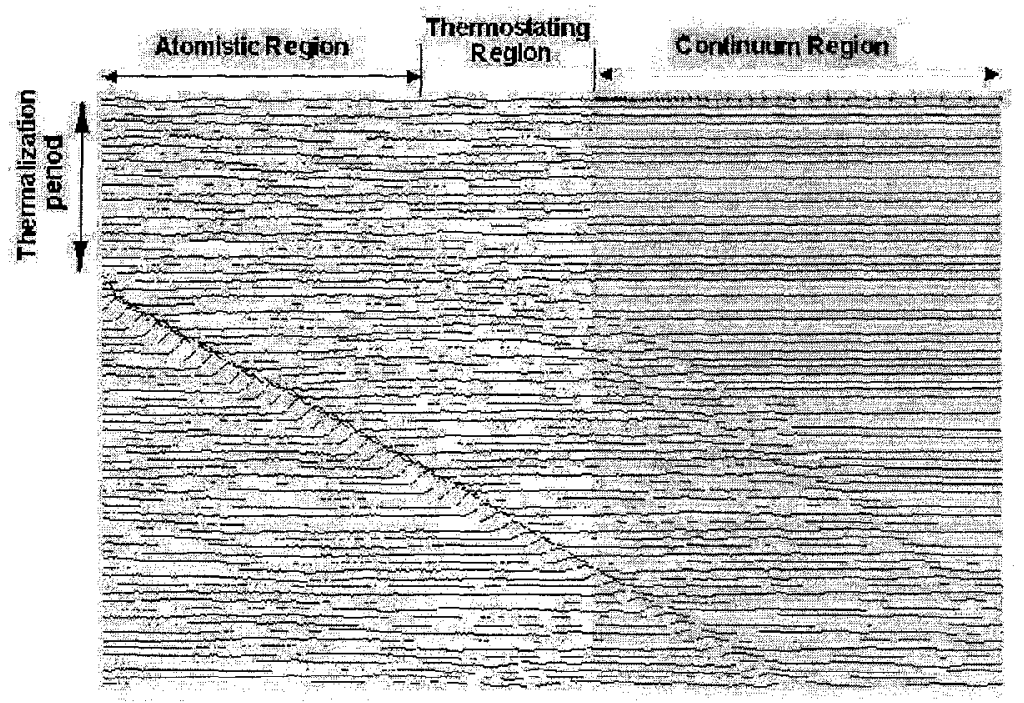

Figure 2.19: Wave propagation. $\lambda=30 \sigma$, damping band $=150 \sigma, Q=0.1$. 


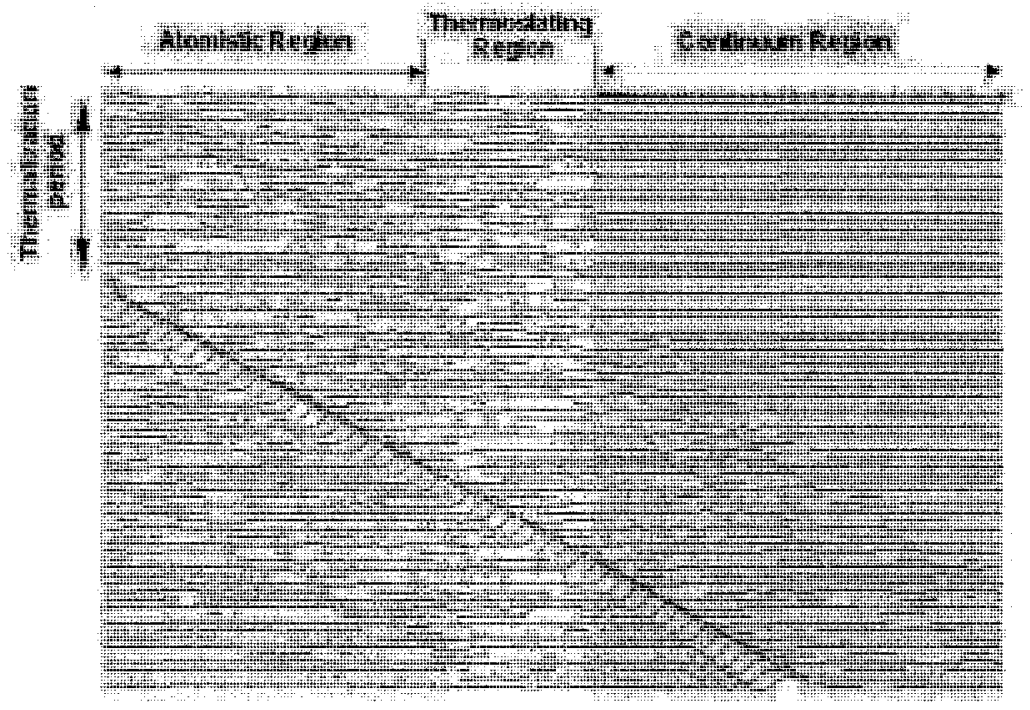

Figure 2.20: Wave propagation. $\lambda=30 \sigma$, damping band $=150 \sigma, Q=10$.

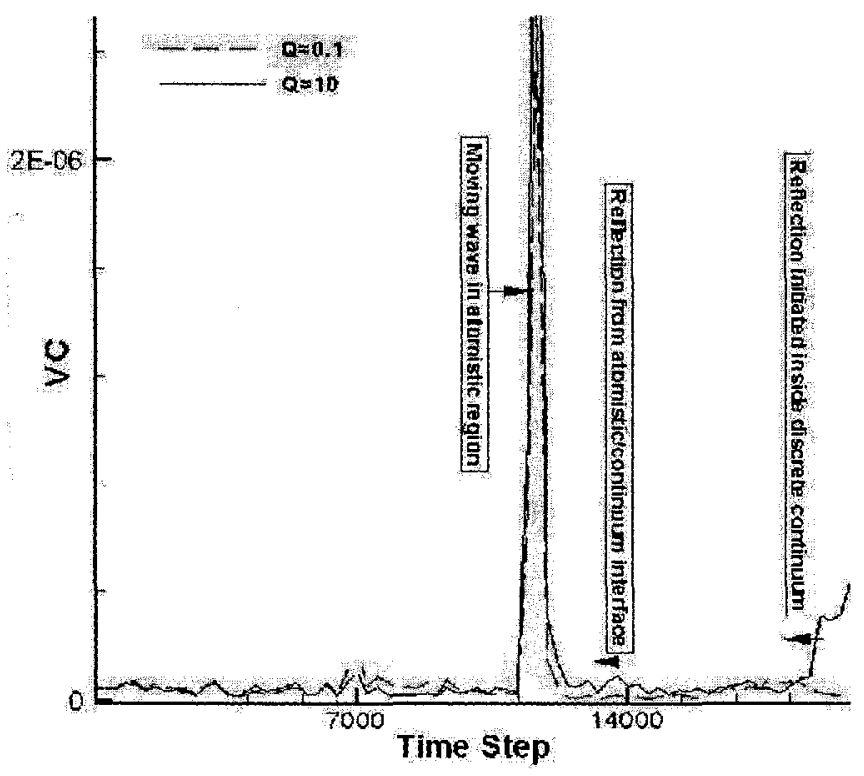

Figure 2.21: magnitude of reflection versus time for two different thermostating masses, $\lambda=14 \sigma$, dissipating band $=150 \sigma$. 


\section{Chapter 3}

\section{Dynamic Coupled Atomistic and Discrete Dislocation Method}

We have discussed in the previous chapters that it is advantageous to implement multiscale modelling schemes in solving problems occurring at several size and time scales, but most of the existing methods have two major limitations associated with them. The first problem is fundamental incompatibility along the interface which presents some difficulties for dynamic approaches and can be considered as their Achilles' heel. We have discussed this issue in Chapter 2 in detail.

The second issue that has to be taken account to carry on a dynamic multiscale is the capability of the method of handling inelastic deformation or plastic flow through the entire coupled model. For some applications such as nanoindentation, the multiscale method must work at the level of the material description by incorporation of both elastic regions and atomistic regions and also at the level of defects, in that the dislocations are treated differently depending on the region in which they reside. A few dynamic multiscale methods such as $Q C[62,63]$ and $C A D D$ methods [44] are capable of modeling of nucleation and motion of dislocations or defects during the simulation.

The $Q C$ method does not allow for the continuum description of a dislocation, and therefore, every dislocation in the $Q C$ model requires a fully atomistic description around its core and slip plane. Hence, computational cost becomes enormous while handling large number of dislocations. At the same time, the $Q C$ method becomes extremely challenging 
in $3 D$ simulations at finite temperature.

The dynamic $C A D D$ model is a new hybrid scheme that extends the capabilities of the existing static model by incorporating dynamic features. The $C A D D$ method also begins by separating the physical problem into spatial regions that are modeled either by a fully atomistic description or by continuum finite elements. The most unique characteristic of the dynamic $C A D D$ method is its ability to accommodate discrete dislocations in the continuum region as well as in the atomistic region. Like other methods, the $C A D D$ model successfully reduces the computational memory and time from what would be required to perform the same simulations using a fully atomistic model. Moreover, the $C A D D$ framework can detect dislocations near the atomistic/continuum interface and pass them across the interface efficiently $[64,1]$.

The dynamic $C A D D$ method consists of five essential components: 1) the atomistic zone, 2) the continuum model, 3) the dynamic coupling between these two regions 4) a dissipating band or thermostating zone, 5) the method for detecting and passing dislocations between these two regions.

\subsection{Dynamic CADD Methodology}

The idea of the coupled atomistic/continuum discrete dislocation method is illustrated in Fig. 3.1. Some details of the method in the static case have been published previously $[64,1]$. The body $\Omega$ can be divided into one (or more) atomistic region $\Omega_{A}$ and one (or more) linear elastic, continuum region $\Omega_{C}$, with interface $\partial \Omega_{l}$ between atomistic and continuum regions. The body is subjected to traction $\boldsymbol{T}=\boldsymbol{T}_{0}$ on $\partial \Omega_{T}$ and displacement $\boldsymbol{u}=$ $u_{0}$ on $\partial \Omega_{u}$. Region $\Omega_{C}$ contains $N_{D}$ continuum discrete dislocations, while the atomistic regions may contain any atomic scale defects (dislocations, grain boundaries, vacancies, voids, amorphous regions). 


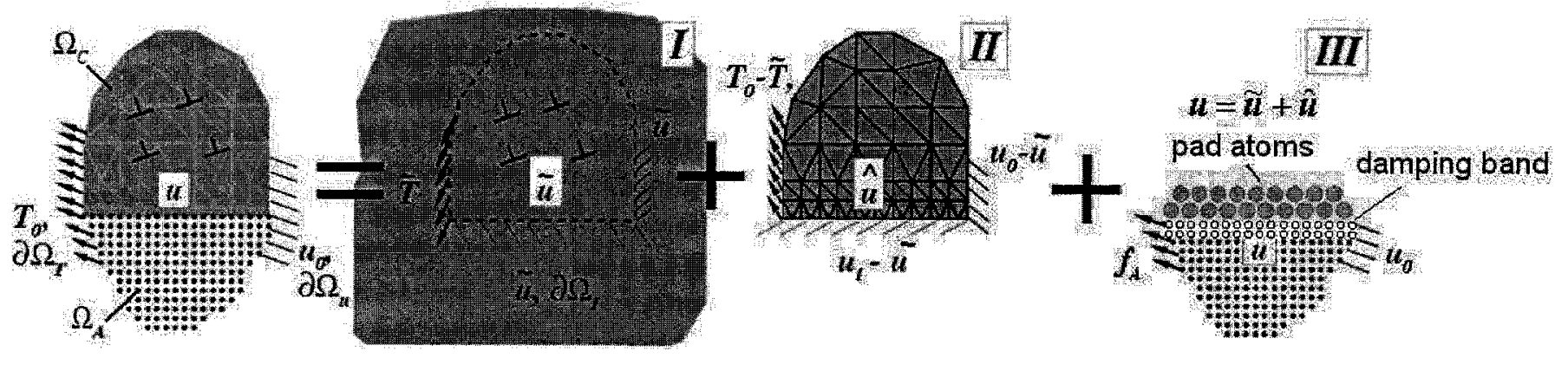


The solution to this boundary value problem consists of atom positions $\boldsymbol{r}_{A}$, dislocation positions $\boldsymbol{d}^{i}$ and continuum displacements $\boldsymbol{u}$, stresses $\boldsymbol{\sigma}$, and strains $\boldsymbol{\epsilon}$. It is obtained by considering separate problems in the continuum (in Fig. 3.1, Problems $I$ and $I I$ ) and atomistic (in Fig. 3.1, Problem III) domains, generated by cutting the system along the interface while enforcing continuity of displacements across $\partial \Omega_{I}$.

\subsubsection{Sub-problem $I I I$}

In dynamic $C A D D$ the atomistic region can be divided into two sections, a free atoms zone and thermostating zone, respectively. In the free atom zone, containing a set of $N_{f}$ atoms with positions $\left(\mathbf{r}_{1}, \ldots, \mathbf{r}_{N_{f}}\right)$, the time evolution of the system is governed by Newton's second law as given in Eq. (2.1). The temperature of the free atoms relies on the applied traction or displacements on the $\Omega_{A}$ boundaries (which do work on the system) and momentum exchanges with the thermostating zone. In the thermostating zone which is a band of atoms adjacent to the atomistic/continuum interface and inside the atomistic region, the kinetic energy is gently tuned about the reference temperature by the use of the Nose-Hoover thermostat as described in the previous chapter. The motion of atoms in this zone is governed by Eq. (2.19). As it has been shown is section 2.3.3, the damping band serves to adequately mitigate the energy reflection overheating of the atomistic region for moderate loading rates by a proper tuning of the thermostating mass, $Q$.

In both zones of the atomistic region, we use the Embedded-Atom Method (EAM) interatomic potentials, although any model providing atomic forces can be used. The $E A M$ is based on density functional theory to calculate ground-state properties of realistic metal systems $[65,66]$. In $E A M$, the energy is expressed as a sum of individual atom energies and the total potential energy, $E_{t o l}$, of a configuration of atoms is written as [65, $66]$

$$
E_{t o t}=\frac{1}{2} \sum_{i} \sum_{j(\neq i)} \Phi_{i j}\left(r_{i j}\right)+\sum_{i} F_{i}\left(\bar{\rho}_{i}\right)
$$

In this expression, $\Phi_{i j}$ is a pair potential evaluated for the interatomic distances $r_{i j}, \bar{\rho}_{i}$ 
is the host electron density at atomic position $i$ due to neighboring atoms, and $F_{i}$ is the embedding energy which is physically interpreted as the energy associated with placing an isolated atom in a uniform electron gas of density $\bar{\rho}_{i}$. The function $\bar{\rho}_{i}$ is found by linearly superposing the electron density from all neighboring atoms via the expression

$$
\bar{\rho}_{i}=\sum_{j(\neq i)} \rho_{j}\left(r_{i j}\right)
$$

where $\rho_{j}$ is the spherically averaged electron density field of an isolated atom. In this and the previous equation, the sums on $j$ are over all atoms within some cutoff radius, $r_{c u t}$ of atom $i$. This cutoff generally includes the first 2 or 3 neighbor distances in the crystal, beyond which it is assumed that atoms do not interact. The ground-state properties, e.g. lattice constant, elastic constants, sublimation energy and vacancy-formation energy of the solid can be calculated from Eq. (3.1) $[65,66]$.

Despite the $2 D$ constraint of the atomistic model, all $M D$ calculations are performed in three dimensions. This corresponds to simulating a slab with periodic boundary conditions applied in the out-of-plane direction that has a thickness equal to the minimum crystallographic repeat distance in that direction. This can be used, for example, to correctly simulate a dislocation whose line is perpendicular to the simulation plane, but not one inclined to it.

\subsubsection{Sub-problem $I+I I$}

The use of continuum representations of defects, achieved by modeling a defect by the macroscopic elastic stress and displacement fields that this defect creates outside of the core region, has been widely successful. The defect field can be deduced from, for example, the Burgers vector of a dislocation or the volume difference of an impurity atom. Defect mobility is then represented by a phenomenological kinetic law relating the velocity or acceleration of the defect to the appropriate configurational force acting on the defect core. The configurational force has contributions from the applied boundary conditions and from the fields of the other defects in the problem. This general approach is 
used by the discrete dislocation $(D D)$ models $[67,68,69]$.

The continuum problem is divided into two complementary problems (Fig.3.1). Problem $I$ consists of dislocations in an infinite elastic continuum and is solved by superposition of the analytical elastic fields due to the individual dislocations at positions $\boldsymbol{d}^{i}$, yielding a total field denoted as the ( $\sim$ ) field. Problem $I$ generates tractions $\tilde{T}$ along $\partial \Omega_{T}$ and displacements $\tilde{u}$ and $\tilde{u}_{I}$ along $\partial \Omega_{u}$ and $\partial \Omega_{I}$ that differ from the values of $\boldsymbol{T}_{0}$ and $\boldsymbol{u}_{0}$ prescribed by the boundary conditions and the $\tilde{\boldsymbol{u}}_{I}$ imposed by the row of atoms defining the edge of the atomistic region. Problem II consists of a linear elastic continuum with no dislocations but subjected to corrective tractions $\hat{\boldsymbol{T}}=\boldsymbol{T}_{0}-\tilde{\boldsymbol{T}}$ on $\partial \Omega_{T}$ and displacements $\hat{\boldsymbol{u}}=\boldsymbol{u}_{0}-\tilde{\boldsymbol{u}}$ on $\partial \Omega_{u}$ and $\hat{\boldsymbol{u}}=\boldsymbol{u}_{I}-\tilde{\boldsymbol{u}}$ on $\partial \Omega_{I}$. All discontinuities and singularities of the dislocations are contained in the ( ${ }^{\sim}$ ) fields of Problem $I$, so the fields of Problem $I I$, denoted as ( ${ }^{`}$ ) fields, are smooth and obtainable numerically by the finite element method.

For problem $I$, the $D D$ approach solves boundary value problem for an isotropic elastic body containing mobile dislocations, which carry the information about plastic deformation. No assumptions about the material plastic constitutive response are necessary, although a set of constitutive rules governing dislocation motion, nucleation and annihilation are required. The $D D$ method has the benefit of being a true mechanism based theory of plasticity; plastic flow arises directly from dislocation motion. The $D D$ formulation models edge dislocations as line defects in an isotropic elastic material, constrained to glide on a fixed slip plane. Long range dislocation interactions occur through their continuum elastic fields. Short range interactions are governed by constitutive rules for dislocation motion, nucleation, and annihilation.

For Problem $I I$, the fully anisotropic, linear elastic constitutive relation is used, so that the continuum elastic constants are matched to those of the crystalline atomistic region. However, for the ( $\sim$ ) field of Problem $I$ deseribing the discrete dislocations, isotropic elasticity with the Voigt-averaged elastic constants are used. Thus the constitutive relations in 
the continuum region are [64]

$$
\begin{gathered}
\tilde{\sigma_{i j}}=2 \mu \tilde{\epsilon_{i j}}+\lambda \tilde{\epsilon_{k k}} \delta i j \\
\hat{\sigma_{i j}}=C_{i j k l} \hat{\epsilon}_{i j}
\end{gathered}
$$

where, $\mu$ and $\lambda$ are the Voigt-average elastic constants found from the fully anisotropic modulus tensor $C_{i j k l}$. Thus, a fully anisotropic solution is obtained when there is no dislocation present in the model, but the introduction of dislocations leads to a more approximate mix of anisotropic and isotropic elasticity.

In the finite element region, the continuum system is divided into elements that are usually much larger than individual atoms in the atomistic region except at the atomistic/continuum interface (see Fig. 3.2). At the interface, the finite elements are scaled down to the atomic size to reduce dynamic impedance mismatching at the interface. Each element is characterized by a sequence of points or nodes on its periphery, and an elastic material which requires the anisotropic elastic moduli, computed from the atomistic model for an appropriate temperature and orientation of a crystal of the material. In particular, the method uses triangular plane-stress elements.

The dynamics of the non-dissipative continuum region discretisized to triangular elements is defined by Eq.(2.37) for the displacements, $\hat{\boldsymbol{u}}$ of a set of $n$ nodes for :

$$
\boldsymbol{M} \ddot{\hat{\boldsymbol{u}}}=-\boldsymbol{K} \hat{\boldsymbol{u}}+\boldsymbol{F}_{e x i}
$$

A lumped-mass scheme is applied in the finite element region. In the lumped-mass approach the mass matrix for each element is taken to be a diagonal matrix, as opposed to the consistent mass matrix which is derived from the weak form of the equations of the motion and is not diagonal. The lumped-mass approach in conjunction with an explicit integration scheme provides a very efficient method to tackle dynamical problems as it discussed in Chapter 2 for $I D$.

The total fields in the continuum are the superposition of the fields from Problems $I$ and $I I: \boldsymbol{u}=\tilde{\boldsymbol{u}}+\hat{\boldsymbol{u}}, \sigma=\tilde{\boldsymbol{\sigma}}+\hat{\boldsymbol{\sigma}}$, and $\epsilon=\tilde{\boldsymbol{\epsilon}}+\hat{\boldsymbol{\epsilon}}$. For a given displacement along 


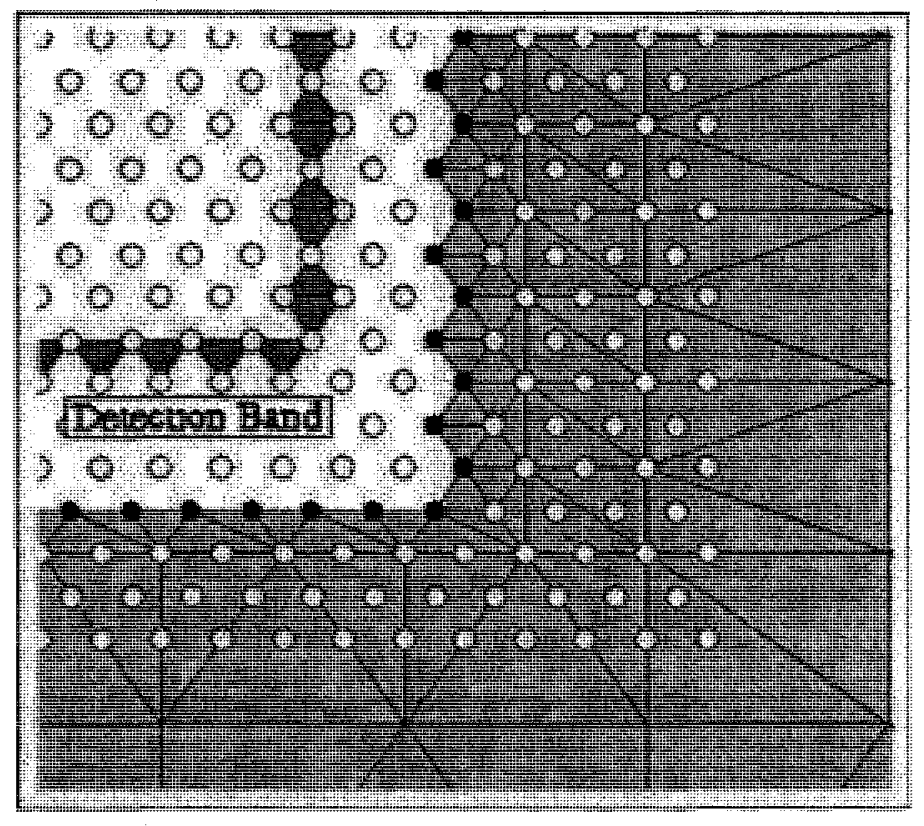

Figure 3.2: Position of the detection band to detect dislocations Dark grey elements are used in dislocation detection. Filled circles are interface atoms and the open circles are the pad atoms that reside on the finite elements.

the atomistic/continuum interface, the stress in the continuum can be used to obtain the Peach-Kohler force on each discrete dislocation. As well, the displacement of any atom in the continuum region can also be obtained using the reference crystal structure, the field $\tilde{\boldsymbol{u}}$, and the interpolated field $\hat{\boldsymbol{u}}$.

To assure that real atoms at and near the interface are properly coordinated, we use a pad of atoms as an intermediary between the local continuum and the non-local, discrete atoms. These pad atoms overlap the edge of the continuum region and deform with it. Forces on the atoms in Problem III are computed based on a physical interpretation of atomic forces in such a way that spurious forces at the atomistic/continuum interface are eliminated [44]. First, the positions of the white pad atoms in Fig. 3.2 are found from interpolation of the continuum displacement fields. Then the forces on the atoms in $\Omega_{A}$ and on $\partial \Omega_{I}$ are obtained from a purely atomistic description of the material including the pad atoms. This pad is at least twice the cutoff radius of the interatomic potentials 
to ensure that atoms on do not see the free surface created by the cut. Use of the pad atoms over a proper overlapping distance has a major role in decreasing the spurious wave reflection from the atomistic/continuum interface [32].

Once the fields in the dislocated solid are known based on a given displacement along the atomistic/continuum interface, the second ingredient is to determine the instantaneous change of the discrete dislocation structure. The important point to note first is that the key quantity involved in constitutive rules for dislocation evolution is the Peach-Koehler force. It can be shown that in the approach outlined above, the component of the Peach-Koehler force in the slip plane can be expressed as [67]:

$$
f^{(k)}=n_{i}^{(k)}\left(\hat{\sigma}_{i j}+\sum_{l \neq k} \tilde{\sigma}_{i j}^{(l)}\right) b_{j}^{(k)}
$$

where $n^{(k)}$ is the slip plane normal and the Burgers vector of dislocation $k . \hat{\sigma}_{i j}$ is the algebraic sum of the external stress and $\tilde{\sigma}_{i j}^{(l)}$ is the stress due to dislocation $l$. This expression highlights the long-range contribution of all other dislocations, through the second term, as well as the image stresses through $\hat{\sigma}$. For two-dimensional simulations involving only edge dislocations, the glide component of the Peach-Koehler force reduces to $f^{(k)}=\tau^{(k)} b^{(k)}$ where $\tau^{(k)}$ is the resolved shear stress on the slip plane. Glide of a dislocation is accompanied by drag forces due to interactions with electrons and phonons. The simplest models of this lead to drag forces that can be expressed as $B v^{(k)}$ where $B$ is the drag coefficient and $v^{(k)}$ is the velocity of the dislocation $k$. When inertia effects of dislocations are ignored, the magnitude of the glide velocity $v^{(k)}$ of dislocation $k$ becomes linearly related to the Peach-Koehler force through [67]:

$$
f^{(k)}=B v^{(k)}
$$

The above relationship is valid when the velocity of dislocation $k$ is smaller than about one fourth of the shear wave velocity [70]. At elevated temperatures, the random thermal vibrations of the atoms would have an additional effect on the moving dislocation. The 
dislocation drag coefficient thus increases with increasing temperature. Temperature dependence of the drag coefficient is not considered during simulations in this thesis, but this dependency is the subject of current investigations.

\subsection{Dislocation Detection and Passing}

\subsubsection{Dislocation Detection}

Detection and moving dislocations between different regions is a unique feature of the $C A D D$ method [1]. Dislocations nucleated in the atomistic region during the loading period may experience driving forces to move from the atomistic region into the continuum region, or vice versa. Because of the seamless nature of the atomistic/continuum interface for elastic deformations, the dislocations do not sense the existence of the interface when the dislocation is a modest distance away. As an atomistic dislocation, i.e. a dislocation with a real atomistic core structure, approaches the interface as shown in Fig. 3.3(a), spurious forces are generated. The elastic continuum is not able to properly accommodate the shear deformation associated with the burgers vector of the dislocation. As the dislocation approaches the interface, the inability of the continuum region to accommodate the displacement discontinuity of the Burgers vector leads to non-physical reflection of the dislocation off of the interface as shown in configuration Fig. 3.3(c), with a residual interface dislocation left behind.

Detection is done by defining a detection band inside the atomistic region at a short distance from the atomistic/continuum interface, as shown in Fig. 3.2. The detection band consists of small triangular elements, with each element sitting on three different slip planes as shown in Fig. 3.4. During the course of a simulation, many dislocations may pass through a certain region or single detection band element and it is necessary to separate out the relevant slip due to each dislocation. The displacements of the three atoms that define the nodes of a detection element will include not only slip but also elastic deformation, rigid body translation, and rotation which must be filtered out in some way. 


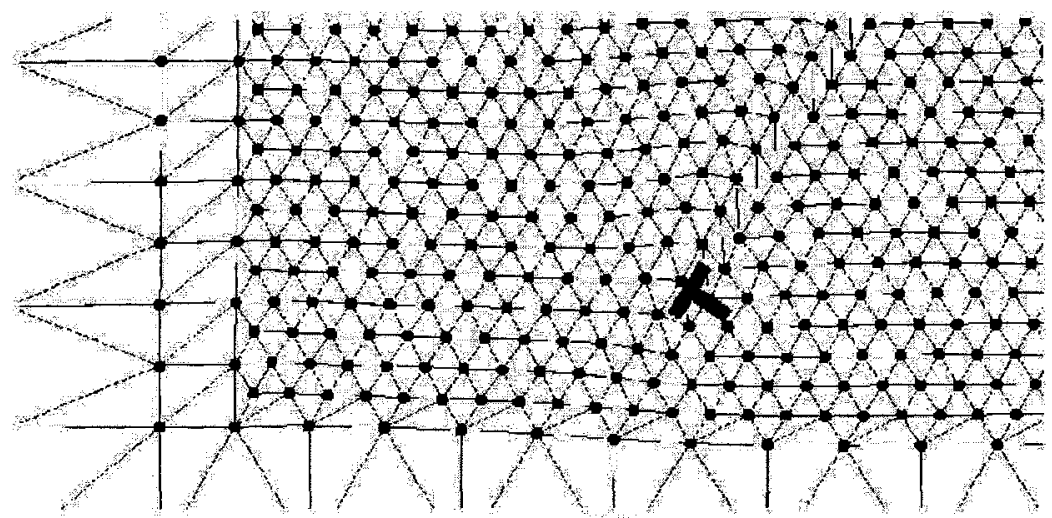

(a)

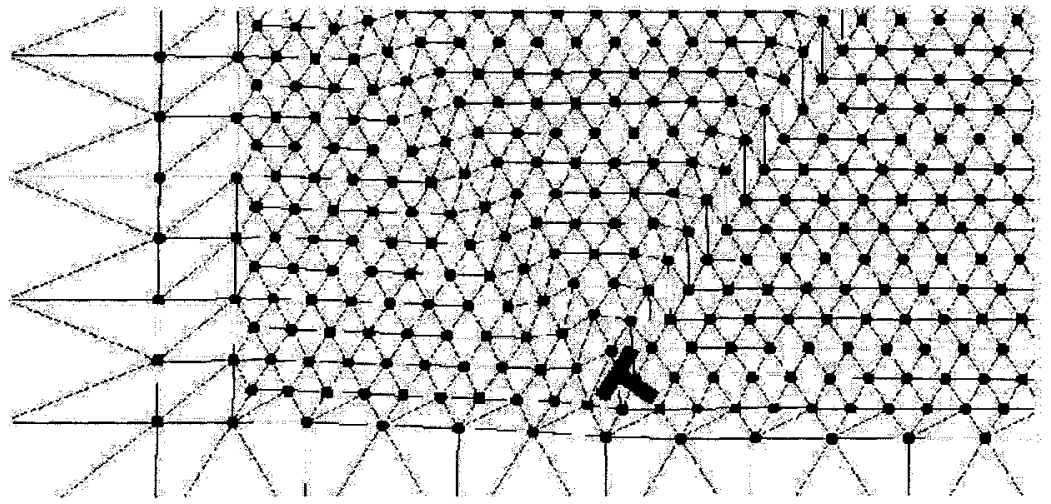

(b)

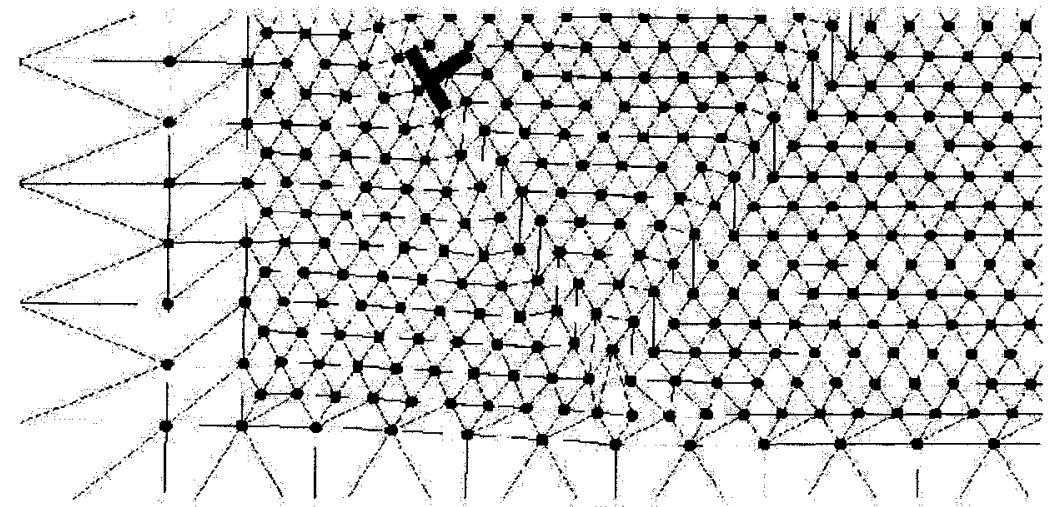

(c)

Figure 3.3: A series of deformation steps illustrating the non-physical reflection of a dislocation that is not allowed to pass across the atomistic/continuum interface. The mesh is drawn between the atoms in the atomistic region for visualization purposes. The deformed elements clearly highlight the slip plane in the wake of the dislocation. 


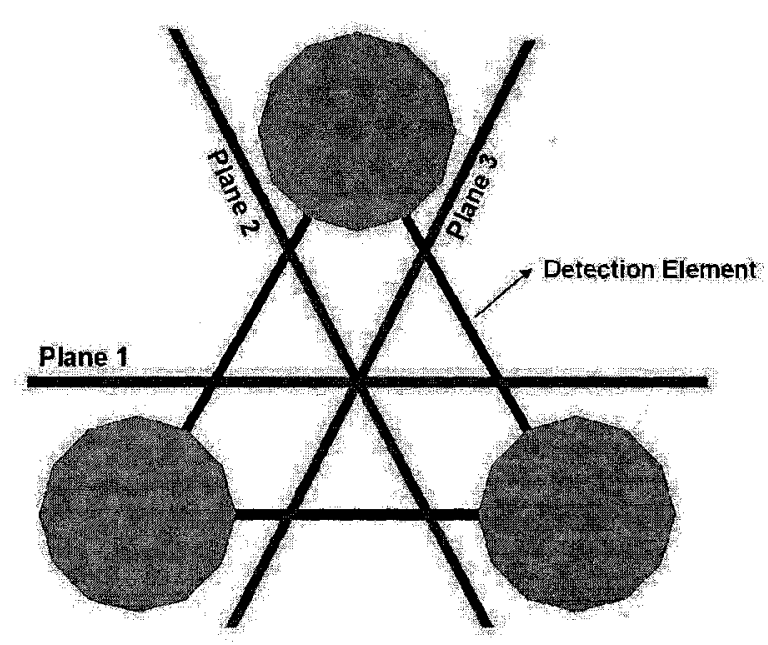

Figure 3.4: A close-up of one detection element, indicating the three slip planes on which it lies.

With these stringent requirements, we find it necessary to make use of the following three key components: (i) all deformation is referred back to the reference configuration of a perfect crystal, i.e. a Lagrangian analysis of deformation is used, (ii) the defined slip measure must be invariant under rigid body rotation and translation of the lattice, i.e. a finite deformation analysis is used, and (iii) only deformation due to new slip is monitored by keeping track of all past slip activity. The first two requirements motivate the use of the local deformation gradient $\mathbf{F}$ and the extraction of the associated Lagrangian plastic (slip) strain tensor $\mathbf{E}^{p}$ for each element. We thus proceed as follows. Each atom has an undeformed position $\mathbf{X}$ and a deformed position $\mathbf{x}=\mathbf{X}+\mathbf{u}$. The displacements due to all previously passed dislocations (known from the current value of $\tilde{\mathbf{u}}$ at each of the nodes defining the detection element) must first be subtracting from the total displacements in the detection band before the elemental strains are computed. Then, the strain due only to new dislocations (plus a smaller elastic contribution) remains. We know all previouslypassed dislocations and their positions in the continuum region. Because dislocations are confined to move in their slip plane in the continuum region (i.e. no cross slip can occur), 

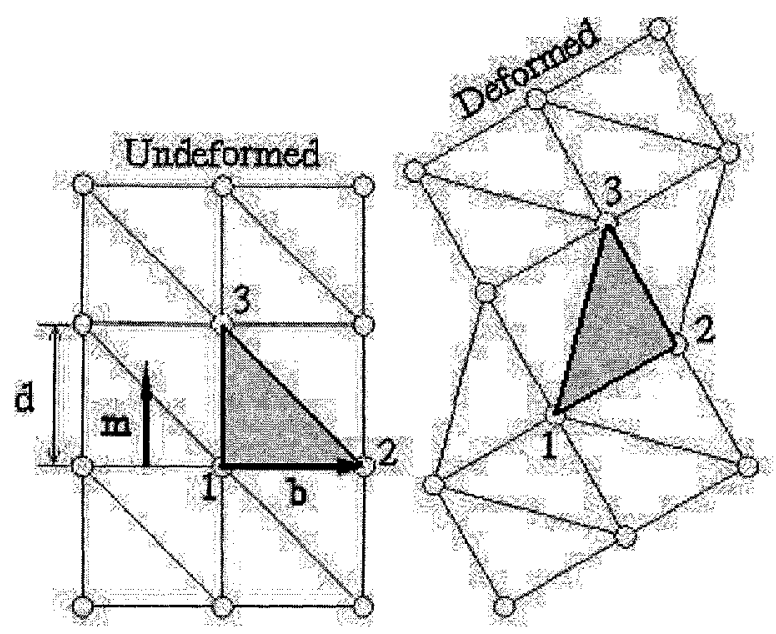

Figure 3.5: Lagrangian finite strain in triangular detection element [1].

the $\tilde{\mathbf{u}}$-field for each continuum dislocation includes a branchcut corresponding to the slip along the atomic planes in the atomic region. Subtracting the $\tilde{\mathbf{u}}$ displacements due to the continuum dislocations, we have the relevant residual displacement $\hat{\mathbf{x}}=\mathbf{X}+\hat{\mathbf{u}}$ where $\hat{\mathbf{u}}=\mathbf{u}-\tilde{\mathbf{u}}$. The deformation gradient $\mathbf{F}$ used to identify new slip is defined as $\mathbf{F}=\frac{\partial \hat{\mathbf{x}}}{\partial \mathbf{x}}$. The deformation gradient can be decomposed into elastic and plastic parts as $\mathbf{F}=\mathbf{F}^{e} \mathbf{F}^{p}$.

An ideal slip deformation $\left(\mathbf{F}^{e}=\mathbf{I}\right)$ is depicted in Fig. 3.5. In the shaded element, nodes 1 and 2 have undergone rigid translation and rotation, while node 3 has moved by one Burgers vector along the slip plane. Since there is no material inside the element, uniform deformation can be assumed and hence the plastic deformation gradient can be decomposed as

$$
\mathbf{F}^{p}=\mathbf{R U}^{p}
$$

where $\mathbf{R}$ is a lattice rotation and $\mathbf{U}^{p}$ is given by

$$
\mathbf{U}_{p}=\mathbf{I}+\frac{(\mathbf{b} \otimes \mathbf{m})}{\mathbf{d}}
$$

where $\mathbf{b}$ is the Burgers vector, $\mathbf{m}$ is the slip plane normal, $\mathbf{d}$ is the interplanar distance shown in Fig. 3.5 and $\otimes$ implies the tensor product. The finite Lagrangian strain of a 
detection band element is

$$
\mathbf{E}=\frac{1}{2}\left[\mathbf{F}^{T} \mathbf{F}-\mathbf{I}\right]
$$

where $\mathbf{I}$ is the identity matrix.

If a dislocation passes along one of planes shown in Fig. 3.4, it generates a Lagrangian finite strain in the element as [1]:

$$
\mathbf{E}^{p}=\frac{1}{2}\left[\left(\mathbf{F}^{p}\right)^{T} \mathbf{F}^{p}-\mathbf{I}\right]
$$

We use $E^{p}$ to detect dislocations as follows. For a given crystal structure and orientation, all the possible slip systems and associated strains $\mathbf{E}^{p}$ are computed by a subroutine. The code is equipped for $F C C$, triangular and $B C C$ crystals. During a simulation, with each update of the atomic positions, we compute the actual strain $\boldsymbol{E}$ in each detection band element, which is efficiently handled using isoparametric, constant strain triangular elements [57]. These actual deformations include elastic deformations so that $\mathbf{F}^{e}=\mathbf{I}$ is not generally satisfied. We therefore identify the slip in each element as being due to that dislocation which minimizes the $L_{2}$ norm of the difference between the actual elemental strain and the dislocation plastic strain:

$$
L_{2}=\sqrt{\left(\mathbf{E}-\mathbf{E}_{i}^{p}\right):\left(\mathbf{E}-\mathbf{E}_{i}^{p}\right)}
$$

If the dislocation minimizing this norm has $\mathbf{b}=0$ then the element is undergoing only elastic deformation and no real dislocation is detected. Otherwise, it is assumed that the core of the detected dislocation is at the centroid of the element under consideration. With the spatial location, Burger's vector, and slip plane all identified by this detection algorithm, the identified dislocation can be passed to the continuum region, as discussed in the next subsection $[64,71]$.

At finite temperature, thermal fluctuations can temporarily make an element appear dislocated. To solve this problem an additional step has been added at finite temperature, we check neighboring elements to be sure the deformation is a correlated one due to real slip. 


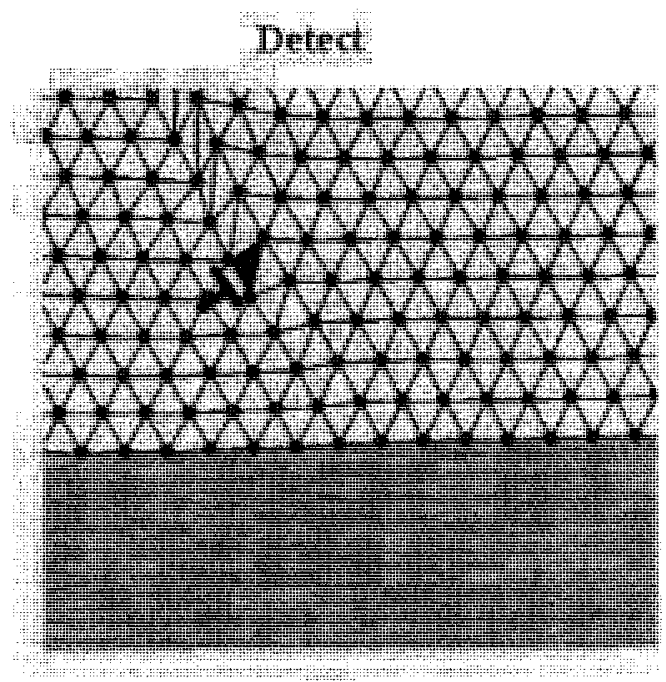

(a)

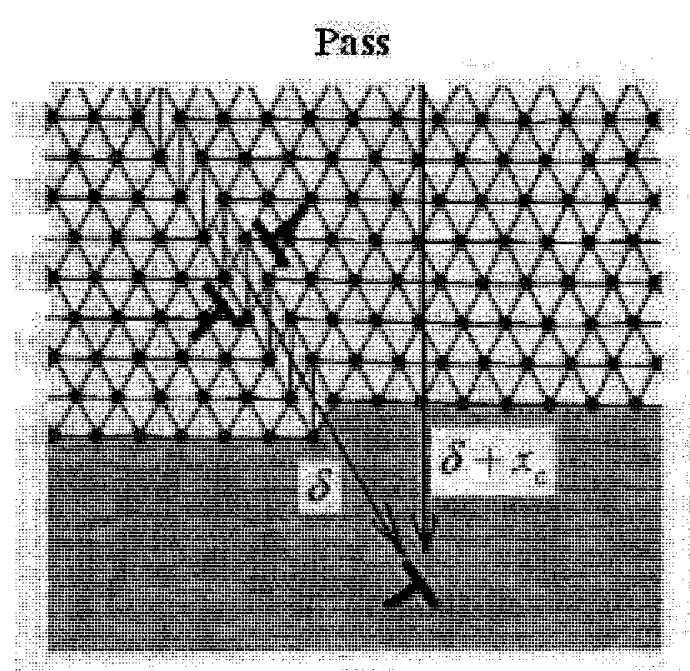

(b)

Figure 3.6: (a) The dislocation is detected at position $x_{c}$ (b)dislocation is passed across the atomistic/continuum interface at position $\delta+x_{c}$

\subsubsection{Dislocation Passing}

After being detected, the dislocation is passed to the continuum as a discrete dislocation.

This is done by artificially shifting the core of the dislocation along its slip plane from its location in the detection band to a location across the interface in the continuum region. At the same time, the continuum elastic displacement of a dislocation dipole is superimposed onto those of the atoms and nodes. These displacements cancel the original core in the atomistic region at $\boldsymbol{x}_{c}$ and replaces it with a continuum dislocation at the position $\boldsymbol{x}_{c}+\delta$ in the continuum region, shown in Fig. 3.6. Once this core is in the continuum region, it is added to the array of discrete dislocations $[64,71]$. The passing procedure in other direction (from the continuum to the atomistic region) is easier to achieve. If the Peach-Koehler force on a dislocation is such that the moving dislocation algorithm (Eq. (3.13), discussed in the next section) attempts to move the dislocation across the interface, this is easily detected geometrically. This dislocation is then inserted into the atomistic region, just inside the detection band, using the superposition of a dipole displacement 
field as outlined previously. The discrete dislocation is kept as an image dislocation so that its branch cut is correctly maintained in the continuum. The dislocation introduced into atomistic region will find the correct atomistic core structure quickly as the next time step proceeds [1].

\subsection{Dynamic Solver}

A dynamic simulation of a $2 D$ coupled atomistic/continuum problem involves integration of the equations of motion, Eq.(2.1), Eq.(3.5), and Eq.(3.7). An apparent similarity between Eq.(2.1) and Eq.(3.5) suggests the possibility for coupling of the dynamic simulation in both atomistic and continuum. Both Eqs.(2.1) and (3.5) are solved simultaneously using the $V V$ integration scheme. This integration method can be started with positions and velocities at time $t$. The total solution procedure of the dynamic $C A D D$ is illustrated in Table 3.1. The detail of the $V V$ solver algorithm is the same as reported in Tables 2.1 and 2.2.

Eq.(3.7) for dislocation glide, on other hand, cannot be treated using the Verlet algorithm. Instead, a straightforward discretization for each time step is used for the simulation as:

$$
u^{(k)}\left(t+\Delta t_{d}\right)=u^{(k)}(t)+\frac{1}{B} f^{(k)} \Delta t_{d}
$$

where $u^{(k)}$ is the $k$ dislocation segment displacement in the glide direction.

Decoupling of Eq.(3.13) from Eqs.(2.1) and (3.5) naturally permits updating of the dislocations on a different timescale $\Delta t_{d}=1000 \Delta t$, using the average $f^{(k)}$ over the 1000 previous time steps, $\Delta t$. The time step used in the simulations is $\Delta t=0.1 p s$. 
Table 3.1: $C A D D$ algorithm.

1. Read as input:

-Elastic Constants of Material, Crystal Structure \& Orientation

-Atomic Potential, Model Size and Geometry

-External Forces \& Boundary Conditions

-Damping Band Width, Initial Temperature,

Equilibrium Temperature \& Thermostating mass

-Time step $(\Delta t)$, Number of Time Steps,

Discrete Dislocation Update Time Step $(N \Delta t)$

2. Set atomic and nodal positions

3. Triangulation (mesh \& detection band generation)

4. Calculate Burger's vectors and dislocation data

5. Give random velocities to atoms to generate initial temperature

6. Set initial conditions

7. Loop over time (t)

\subsection{Set thermostat}

7.2 Integration of $M D$ and $F E$ equations (free atoms \& thermostating atoms)

7.3 Coupling (Update pad atoms displacements \& positions)

7.4 Pass detected dislocations

7.5 Update position of discrete dislocations if $t=N \Delta t$

7.6 Output results for selected time step

8. End 


\section{Chapter 4}

\section{Nanoindenation of ductile crystals}

Nanoindentation has received considerable attention in recent years, owing in part to its practical applications as an experimental tool of probing surface mechanical properties of materials at the micro/nanoscale. On the fundamental side, nanoindentation offers a unique opportunity for studying defect nucleation and interactions from both experimental and theoretical viewpoints. However, nanoindentation experiments are difficult to interpret at a fundamental level because of the involvement of multiple length scales. There are long-range elastic interactions between the defects and short-range atomic-scale processes involving the arrangements of atoms. Both the short-range and long-range properties can affect material response during indentation [14].

It is known that temperature can have a significant effect on material response. Hence, performing simulations of nanoindentation process at finite temperature can provide researchers the opportunity to understand the fundamental details of the contact problem and material properties as a function of temperature.

In this chapter, we apply the dynamic $C A D D$ method to simulate the nanoindentation process. We will show that the technique overcomes two major problems in nanoindentation simulations: superheating of the atomistic region because of phonon (or high frequency wave) reflection from the atomistic/continuum interface and the long-range motion of dislocations.

The effectiveness of the tool is tested for both visualization and quantification of dis- 
location nucleation and motion beneath the indenter, including the evolution of surface deformation resulting from defect nucleation. The results reported in this chapter provide direct and fundamental mechanistic insights into the processes associated with contact deformation, and quantitative predictions of discrete plasticity events that are consistent with some experimental observations of nanoindentation in single crystals [72]. A comprehensive parametric study has been conducted to identify the relationship between initial temperature of the substrate and temperature rise during a nanoindentation process, and to characterize the mechanical behavior of the material as well as the dislocation nucleation and propagation inside the substrate. The size-dependency of the material hardness and rate-dependency of dislocation nucleation are studied.

\subsection{Indentation at the nanoscale}

The history of indentation as a tool to quantify materials properties goes back to the 1900 s. Brinell began to investigate indentation of metals by spherical indenters. He noticed that indentation could be an alternative to the uniaxial tensile test to compare various steels. He defined hardness as peak load over the area of indention which indicates the testing material's resistance to indentation. Indentation testing offered significant advantages over uniaxial testing. It was no longer necessary to fabricate large tensile specimens and analysis of very small and very thin samples was possible. In the early part of the last century, it was found in uniaxial tests that below a critical value of stress called the critical resolved shear stress, deformation is elastic and recoverable. Above this limit, permanent plastic deformation occurs. The plastic deformation was explained later in terms of dislocations [73]. Nucleation and accumulation of dislocation were used to explain some observations like increasing yield stress with increasing amounts of strain or work hardening. Atkins and Tabor observed that indentions relax elastically upon unloading and thus can be put in terms of Hertz's elastic equations [74]. These equations were then modified and used to calculate the elastic modulus from an indentation experiment. In the middle of the last 
century, large steel spherical indenters were replaced by small pyramidal diamond indenters, allowing indentations on the order of microns [75]. These microindentation tests were capable of probing the deformation properties of grain boundaries, different phase regions, and surface-localized work hardening.

In the last twenty years, miniaturization of instruments has allowed probes to measure material properties of semiconductor devices as well as thin films and coatings [76]. The key development has been continuous monitoring of displacement and load during the indentation. In principle, load-displacement data can then be used to derive properties without actual direct measurement of the contact area, which is difficult to obtain due to the small size of the indentations. Information from nanoindentation can be used in parallel with various microscopy techniques such as atomic force microscopy, scanning electron microscopy and x-ray topography to reach into a deeper understanding of the relationship between mechanical properties and various phases, structures, and defects at the nanoscale [76].

\subsection{Elastic Modulus and Hardness Measurement}

The nanoindentation test relies on the continuous recording of force and displacement as the indenter penetrates the substrate. The resulting force-displacement curves are used to determine the mechanical properties, most typically hardness and elastic modulus [75]. A schematic diagram of a typical force-displacement curve is shown in Fig. 4.1. Hardness is normally defined as a mean pressure $P_{m}$ given by

$$
P_{m}=H=\frac{P_{t}}{A}
$$

where $P_{t}$ is the maximum load or force during an indentation cycle while $A$ is the projected area of the indentation at the maximum load.

The slope of the curve, $d P / d h$, upon unloading is indicative of the stiffness of the contact as shown in Fig. 4.1. This value generally includes a contribution from both the 


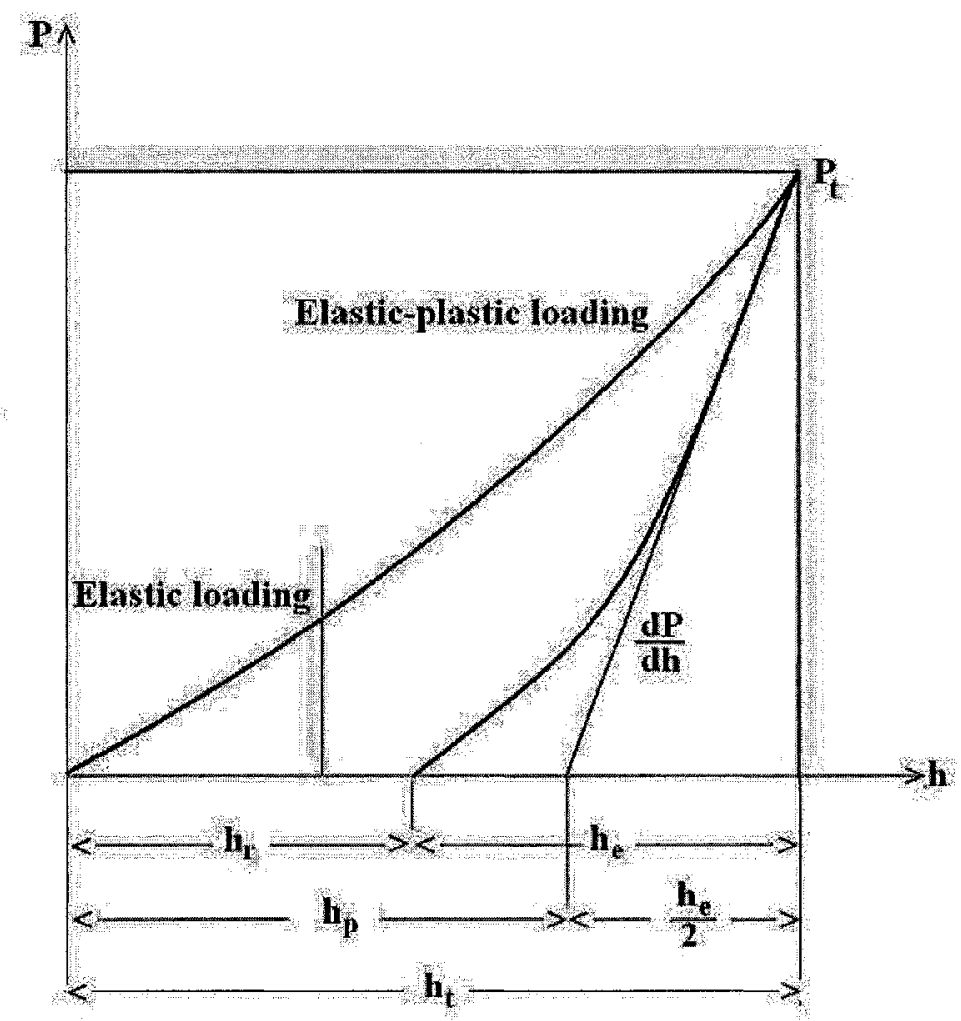

Figure 4.1: Typical load-displacement curve

material being tested and the response of the test device itself. The stiffness of the contact can be used to calculate the reduced modulus of elasticity $E_{r}$ as:

$$
E_{r}=\frac{S}{2} \frac{\sqrt{\pi}}{A}
$$

where $S$ is the unloading stiffness or initial slope of the unloading curve, and $A$ again is the projected contact area at maximum load. The reduced modulus is also related to the modulus of elasticity of the test specimen through the following relationship from contact mechanics

$$
\frac{1}{E_{r}}=\frac{1-v_{s}^{2}}{E_{s}}+\frac{1-v_{i}^{2}}{E_{i}}
$$

where $v_{s}, v_{i}$ and $E_{s}, E_{i}$ are Possion's ratio and modulus of the sample and indenter respectively. 
In turn, for rigid indenters, the indentation modulus $E_{r}$ is a function of only the elastic constants of the indented substrate. In this case, $E_{r}$ is not a material property but rather a snapshot of the solid stiffness. For instance, in the isotropic case, $E_{r}$ reduces to the plane-stress elastic modulus,

$$
E_{r}=\frac{E_{s}}{1-v^{2}}=\frac{C_{1111}^{2}-C_{1122}^{2}}{C_{1111}}
$$

where $C_{1111}$ and $C_{1122}$ are the forth order stiffness tensor coefficients of the substrate. For general anisotropic materials, no such straight forward formula is available.

While a value for $S$ can be obtained easily from experimental force-displacement curves as indicated in Fig. 4.1, the projected area during indentation cannot be easily measured. In experiments, the dimensions of a remaining impression are measured optically after unloading. The projected area of contact can be typically estimated using an empirically determined shape function $A=f\left(h_{p}\right)$, where $h_{p}$ is defined as the depth of plastic contact. Several methods with varying degrees of accuracy have been proposed. Field and Swain suggested a very crude model for spherical indenters based solely on geometrical considerations [77]. To a first approximation, the depth of plastic contact $h_{p}$ may be estimated as

$$
h_{p}=\frac{h_{r}+h_{t}}{2}
$$

where $h_{t}$ is the maximum displacement and $h_{r}$ is the depth of the residual impression after the load is removed. In the case of a spherical indenter of radius $R$, the depth of plastic contact is then used to estimate the radius of contact $a$ of the indenter

$$
a=\sqrt{2 R h_{p}-h_{p}^{2}}
$$

The projected contact area is given as $A=\pi a^{2}$. Oliver and Pharr [78] proposed a more robust approximation that accounts for the curvature of the unloading curve and provides a physically justifiable estimation for the depth of plastic contact. In this classic model, the depth of plastic contact is given by

$$
h_{p}=h_{t}-\epsilon \frac{P_{\max }}{S}
$$


where again $h_{t}$ is the maximum displacement, $P_{\max }$ is the maximum load and $S$ is the unloading stiffness. The constant $\epsilon$ is a geometrical factor related to the deflection of the surface at the contact perimeter that depends on the indenter geometry. For a shallow indentation with a spherical indenter $\epsilon$ is equal to 0.75 . For a parabola of revolution or spherical indenter, $h_{p}$ may be substituted into Eq. 4.6 to determine the radius of contact and thus the projected contact area $A$.

\subsection{Model System and Simulation Method}

\subsubsection{Material selection}

For our research, we select a $2 D$ triangular lattice of aluminum. The triangular structure is not a real crystal structure, and it is unstable both in $3 D$ and out-of-plane deformation. It is stable in $2 D$ because it is constrained in the $z$ direction which allows us to get $2 D$ in-plane deformation. Another feature of such a $2 D$ triangular structure is that, a dislocation gliding on one of the three slip systems can dissociate into two dislocations, one on each of the other two systems. Such dissociation can be viewed as a $2 D$ analogue to cross-slip in $3 D$ [79]. Also, the dislocation character can only be edge with its line direction perpendicular to the $x-y$ plane of the model, simplifying the details of dislocation detection and passing. Moreover, the elastic properties are exactly isotropic in-plane for triangular lattice. These features of triangular structure motivate us in using such a model material for our simulation. Our simulations cannot be used, therefore, to make accurate quantitative predictions about real materials. Instead, they permit us to examine trends and generic behaviors that real materials may also exhibit.

A EAM potential [7] is used to model the substrate material. From the initial configuration of the atoms, the Bravais lattice vectors of the substrate material is illustrated in 


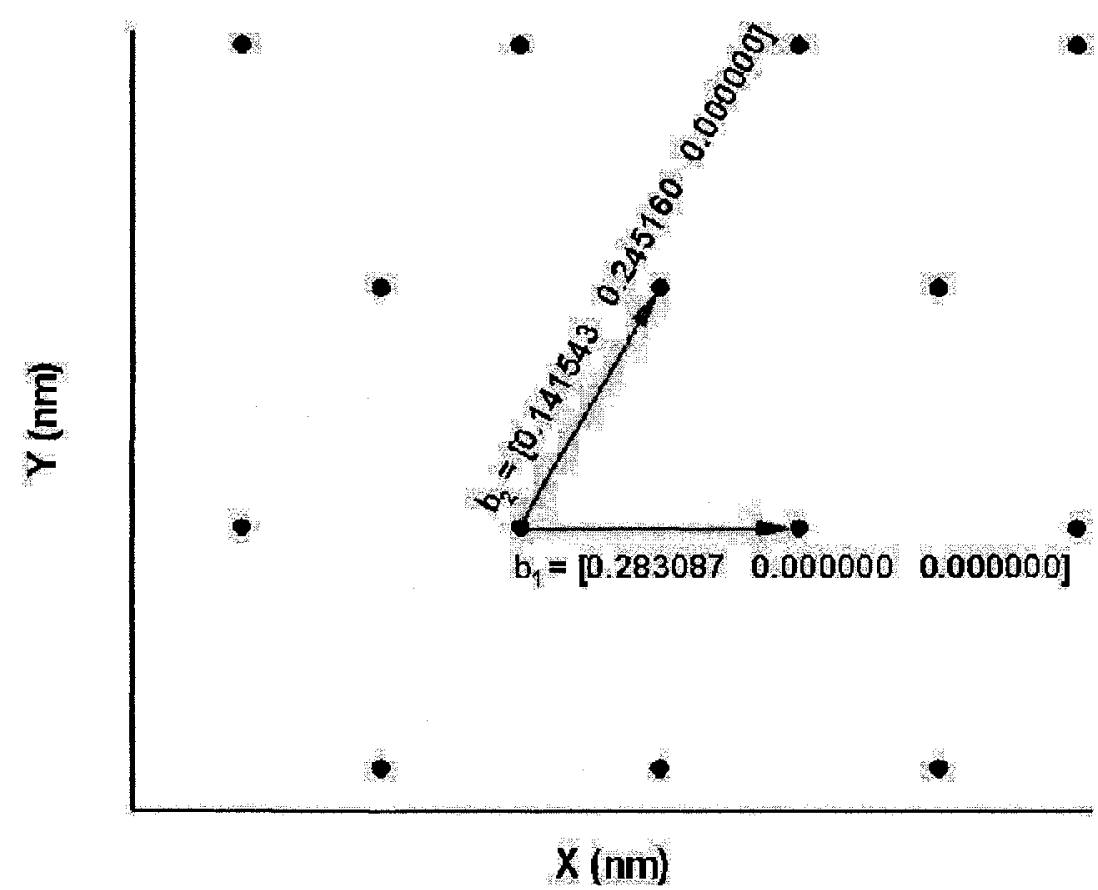

Figure 4.2: Baravais lattice vectors of triangular Aluminum

Fig. 4.2. For triangular Al, the equilibrium Bravais vectors are:

$$
\begin{aligned}
& b_{1}=\left[\begin{array}{lll}
0.283087 & 0.000000 & 0.000000
\end{array}\right] \\
& b_{2}=\left[\begin{array}{lll}
0.141543 & 0.245160 & 0.000000
\end{array}\right] \\
& b_{3}=\left[\begin{array}{lll}
0.000000 & 0.000000 & 0.295738
\end{array}\right]
\end{aligned}
$$

As the above Bravais vectors show, all the simulations in this dissertation are two dimensional, but with a fully three-dimensional underlying crystal lattice. This is achieved by employing periodic boundary conditions in the $z$ (out-of-plane) direction and a minimal z-dimension of the periodic cell to correctly produce the crystal structure.

In the dynamic $C A D D$ formulation, a fully anisotropic and linear elastic constitutive relation is used for the $\left(^{\wedge}\right)$ fields of Problem $I I$, that describes the finite element solution of the total continuum field, to ensure a match of elastic constants in the continuum region to 
those of the crystalline atomistic region. However, for the ${ }^{\sim}$ fields of Problem I describing the discrete dislocation, we use the analytic fields from isotropic elasticity with Voigtaveraged elastic constants. The constitutive relations in the continuum region are shown in Eq.(3.3) and Eq.(3.4). The macroscopic elastic moduli $C_{i j k l}$ in Equation 3.4 can be written in terms of the microscopic interatomic interactions. At equilibrium, these moduli take the form [65]

$$
C_{i j k l}=\left(B_{i j k l}+F^{\prime}(\bar{\rho}) W_{i j k l}+F^{\prime \prime}(\bar{\rho}) V_{i j} V_{k l}\right) / \Omega_{0},
$$

where $\Omega_{0}$ is the undeformed atomic volume, and

$$
\begin{aligned}
B_{i j k l} & =\frac{1}{2} \sum_{m}\left(\Phi_{m}^{\prime \prime}-\Phi_{m}^{\prime} / a^{m}\right) a_{i}^{m} a_{j}^{m} a_{k}^{m} a_{l}^{m} /\left(a^{m}\right)^{2} \\
W_{i j k l} & =\sum_{m}\left(\rho_{m}^{\prime \prime}-\rho_{m}^{\prime} / a^{m}\right) a_{i}^{m} a_{j}^{m} a_{k}^{m} a_{l}^{m} /\left(a^{m}\right)^{2} \\
V_{i j} & =\sum_{m} \rho^{\prime} a^{m} a_{i}^{m} a_{j}^{m} / a^{m} .
\end{aligned}
$$

where, $\Phi_{m}^{\prime \prime}=\left[d^{2} \Phi(r) / d r^{2}\right]_{r=a^{m}}, \rho_{m}^{\prime \prime}=\left[d^{2} \rho(r) / d r^{2}\right]_{r=a^{m}}$, m indicates the sum over the neighbors of one atom in the crystal and $\Phi, \rho$ and $F$ were defined for $E A M$ in Eq. 3.1.

This expression for the elastic modulus demonstrates an important connection between atomistic and continuum models and are used for the finite element problem. This expression will yield three independent non-zero components of the moduli for a crystal with cubic symmetry. In Voigt notation they are expressed as follows [65]

$$
\begin{array}{r}
C_{1111}=C_{11}=\left[B_{11}+F^{\prime}(\bar{\rho}) W_{11}+F^{\prime \prime}(\bar{\rho})\left(V_{11}\right)^{2}\right] / \Omega_{0}, \\
C_{1122}=C_{12}=\left[B_{12}+F^{\prime}(\bar{\rho}) W_{12}+F^{\prime \prime}(\bar{\rho})\left(V_{11}\right)^{2}\right] / \Omega_{0}, \\
C_{1212}=C_{44}=\left[B_{12}+F^{\prime}(\bar{\rho}) W_{12}\right] / \Omega_{0},
\end{array}
$$

As it mentioned before, the triangular aluminum is oriented with the $\mathrm{z}$-axis out-ofplane, so as to create an effectively $2 D$ material. Using the $E A M$ potential, the calculated in-plane elastic constants of aluminum is presented in Table 4.1. 


\begin{tabular}{|c|l|}
\hline$C_{11}=C_{22}$ & $0.9581 \mathrm{eV} / \AA^{3}=153.5 \mathrm{GPa}$ \\
\hline$C_{12}=\lambda$ & $0.5747 \mathrm{eV} / \AA^{3}=92.08 \mathrm{GPa}$ \\
\hline$C_{66}=\mu$ & $0.1917 \mathrm{eV} / \AA^{3}=30.71 \mathrm{GPa}$ \\
\hline
\end{tabular}

Table 4.1: Calculated in-plane elastic constants for aluminum.

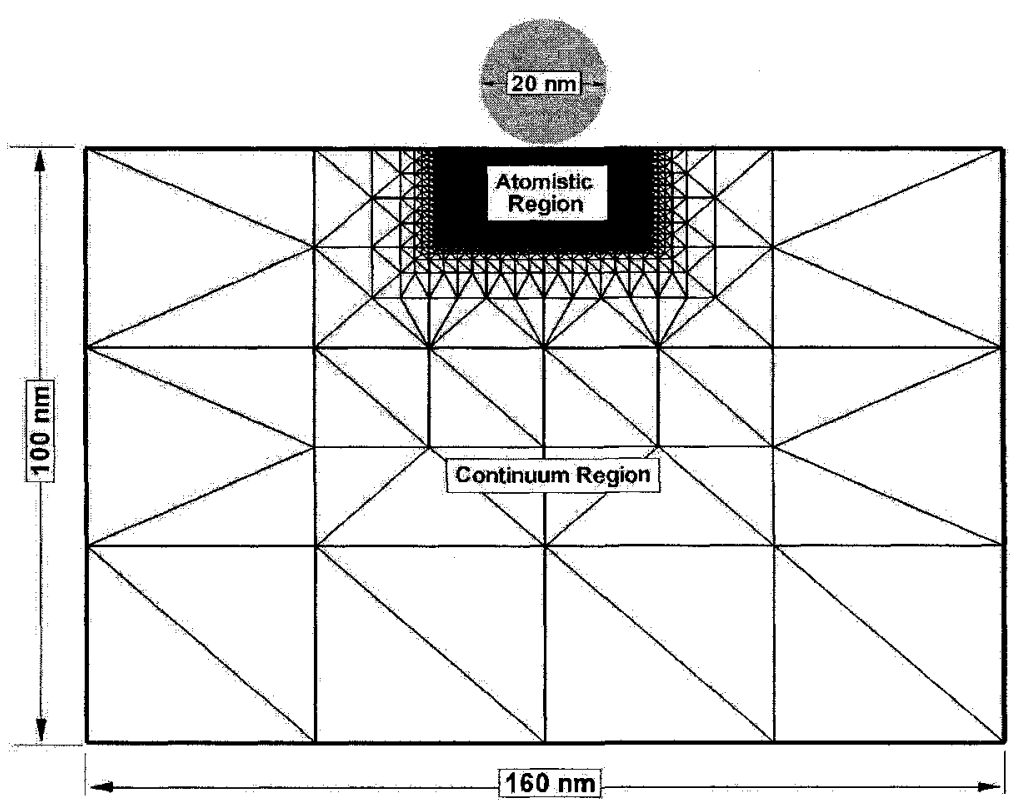

Figure 4.3: Multiscale model geometry for nanoindentation simulation.

\subsubsection{Multiscale model description}

The model of the indentation problem along with the boundary conditions and the mesh used in the computations are shown in Fig. 4.3. A rigid cylindrical indenter is driven into a block of triangular aluminum with c-axis normal to the in-plane deformation. The region close to the indentation site is simulated with atomic resolution. In the atomistic region, every atom is explicitly represented and treated using EAM interatomic potentials [65]. The remaining regions are simulated in a continuum sense, by finite elements. Fig. 4.4 also shows the details of the matching zone which is used to make a proper link between 
the atomistic and continuum regions. At the interface between the $F E$ nodes and the atoms there is a one-to-one correspondence between atoms and nodes on the FE mesh. Moving away from the interface on the continuum side, the $F E$ nodes become increasingly sparse and the corresponding elements become larger so as to always completely fill the physical space. As it is shown in Fig. 4.4, there is a pad region where pseudoatoms exist on the continuum side of the interface and overlap physical space with the FEs. Some of the pad atoms coincide with the $F E$ nodes while others lie within the elements; their displacements are dictated by the interpolated $F E$ displacements. Finally, a damping band is considered along the atomistic/continumm interface inside the atomistic region. The thickness of the damping band is $2.5 \mathrm{~nm}$.

As Fig. 4.3 shows, the multiscale model allows for consideration of a large specimen without a significant increase in the size of the problem. Hence, the boundary of the model can be remote enough from the process region (indentation site) such that the boundary conditions do not influence the results, while at the same time long-range dislocation motion is supported. The application of the $C A D D$ technique for modeling the given sample shown in Fig. 4.3 permits the modeling of more than 200,000 atoms using only 2800 atoms and 200 nodes. Periodic boundary conditions are used in the out-of-plane direction.

Historically, different atomistic models are used to study indentation and retraction (e.g. $E A M$ potentials [80], other semi-empirical potential models [81], or first-principle methods [82]). These calculations have shown a strong bonding between the indenter tip and the surface because of the large amount of adhesion energy between two clean surfaces, causing a jump to contact when the indenter approaches and a necking between the tip and surface during retraction. However, tip-surface adhesion interaction was found to be reduced dramatically in experiments because each was not atomically clean or their surfaces were passivated by the addition of an alkanethiol layer which prevents the bonding [83]. 


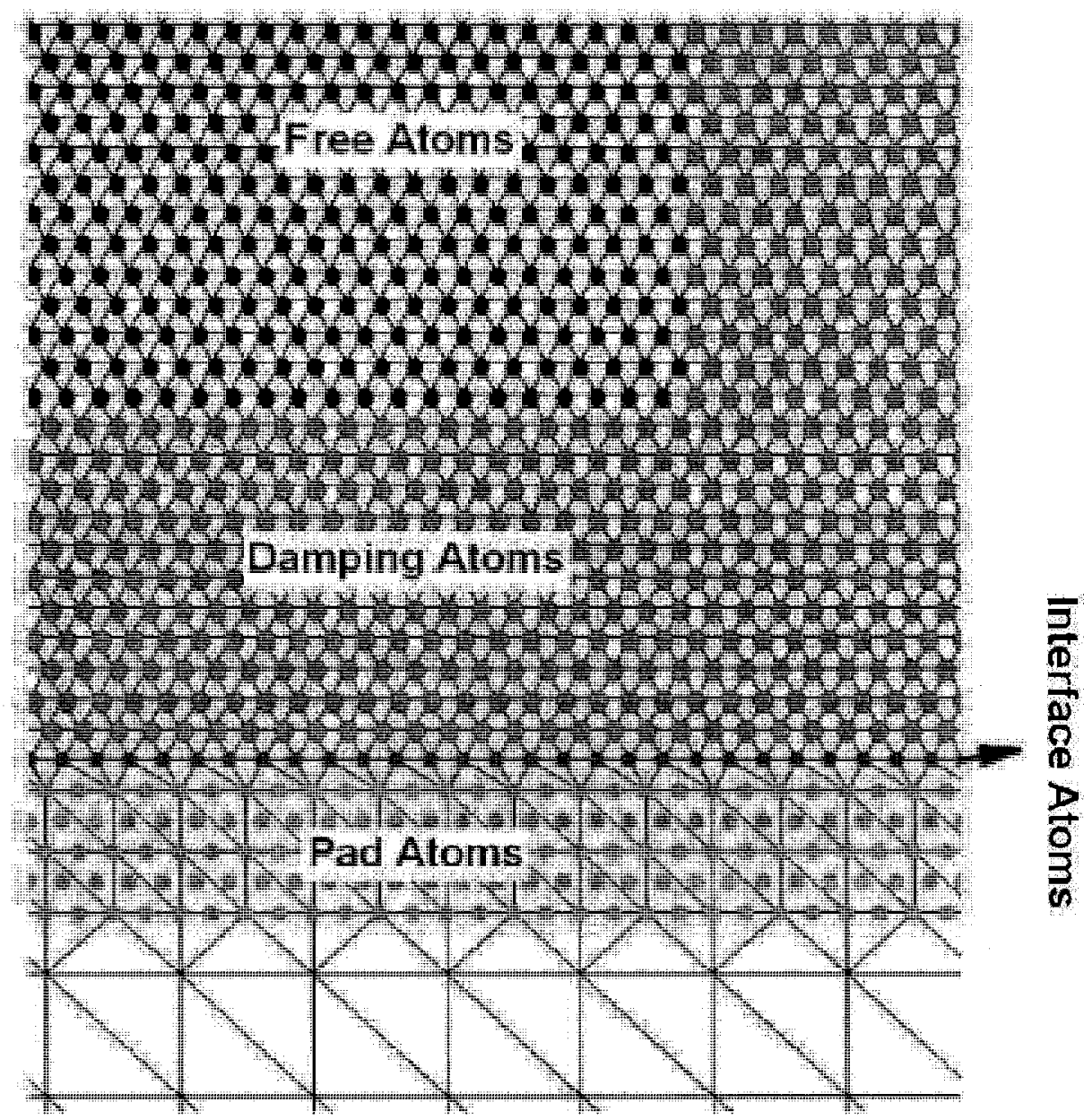

Figure 4.4: A close-up of the atomistic/continuum interface which shows continuum elements, free, pad and damping atoms as well as interface atoms. The circles that lie inside the continuum region are used as a "pad" of atoms to couple the atoms to the continuum region. The grey elements inside the atomistic region are detection elements.

In this study, a strongly repulsive potential model between the indenter tip and the metal surface is designed to simulate the passivated surface. Each atom in the indented material interacted with the idealized spherical (or cylindrical) indenter via a repulsive potential. In the repulsive potential formulation for the virtual indenter, the indenter acts as if it were a giant atom with a repulsive force between the indenter and any atoms that 


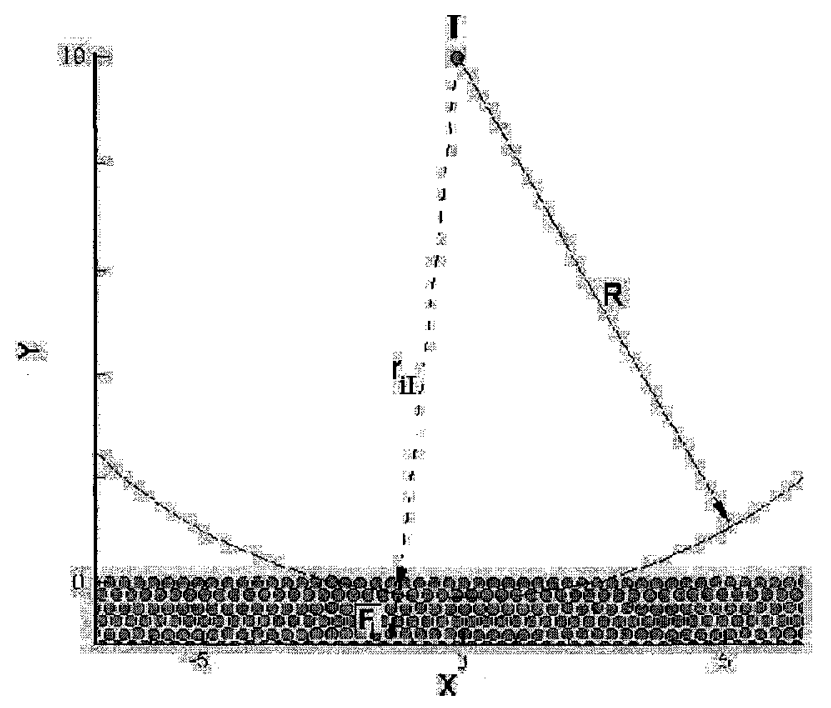

(a)

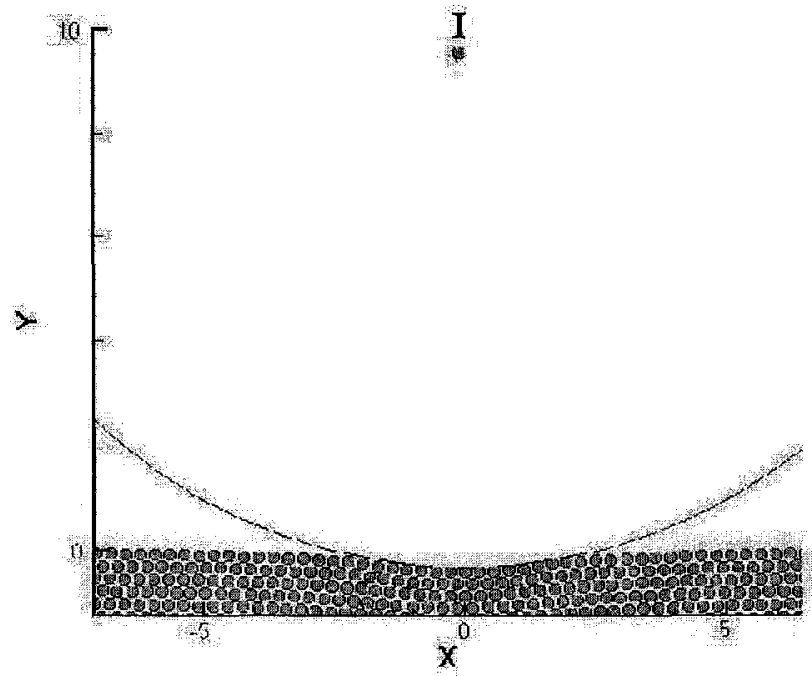

(I)

Figure 4.5: Schematic diagram of the repulsive indenter. a) a normal force is applied normal to the surface of the indenter, $b$ ) displacement of atoms in contact with the indenter which depends on constant $C$ in Eq. (4.15)

the indenter contacts. The repulsive force from the indenter $I$ on substrate atom $i$ can be 
derived from the following indenter potential

$$
\Phi= \begin{cases}C\left(r_{i I}-R\right)^{2} & \text { if } r_{i I}<R, \\ 0 & \text { if } r_{i I} \geq R\end{cases}
$$

where $R$ defines the radius of a virtual spherical (or cylindrical) indenter, $r_{i l}$ is the radial distance from the center of the indenter to atom $i$ as shown in Fig. 4.5. $C$ is constant related to the stiffness of the indenter. The total force on atom $i$ is then:

$$
F_{i}=F_{E A M}+F_{i I}
$$

where $F_{E A M}$ is the total force determined by the embedded atom method and $F_{i I}$ is the force on atom $i$ from the indenter. To simulate loading or unloading of the indenter, the center of the indenter is placed above the substrate in preparation for the indention. As shown in Fig. 4.5, it is centered in the horizontal direction with respect to the boundaries of the system. The vertical direction for the center of the indenter is then given as the minimum $y$ coordinate of the substrate atoms plus the radius of the indenter. This initial placement of the indenter ensures that the indenter is in contact with the surface at the start of the simulation. To load or unload the substrate, the $y$ coordinate of the indenter is decreased or increased by $I_{\text {indent }} / n_{\text {steps }}$ at each time step where $n_{\text {steps }}$ is the number of time steps required to indent to a depth of $I_{\text {indent }}$. The force on the indenter is then calculated by summing the forces normal to the surface for all atoms in contact with the indenter $\left(r_{i I} \leq R\right)$. The force data along with the displacement of the indenter are used to generate force-displacement curves.

Lilleodden et. al [84] have studied the effect of the indenter stiffness on the repulsive function stated by Eq. (4.15). They optimized the value of $C$ by conducting a conjugate gradient energy minimization at each indentation step at zero temperature. We carried out several test simulations to quantify the indenter repulsive function for the dynamic indentation. Our tests show that the small values of $C\left(\leq 15 \mathrm{~N} / \mathrm{m}^{2}\right)$ represents a very soft indenter which leads to negligible plastic deformation of the substrate. On the other hand, large values of $C\left(\geq 2500 \mathrm{~N} / \mathrm{m}^{2}\right)$ can cause severe bouncing of the surface atoms as they 


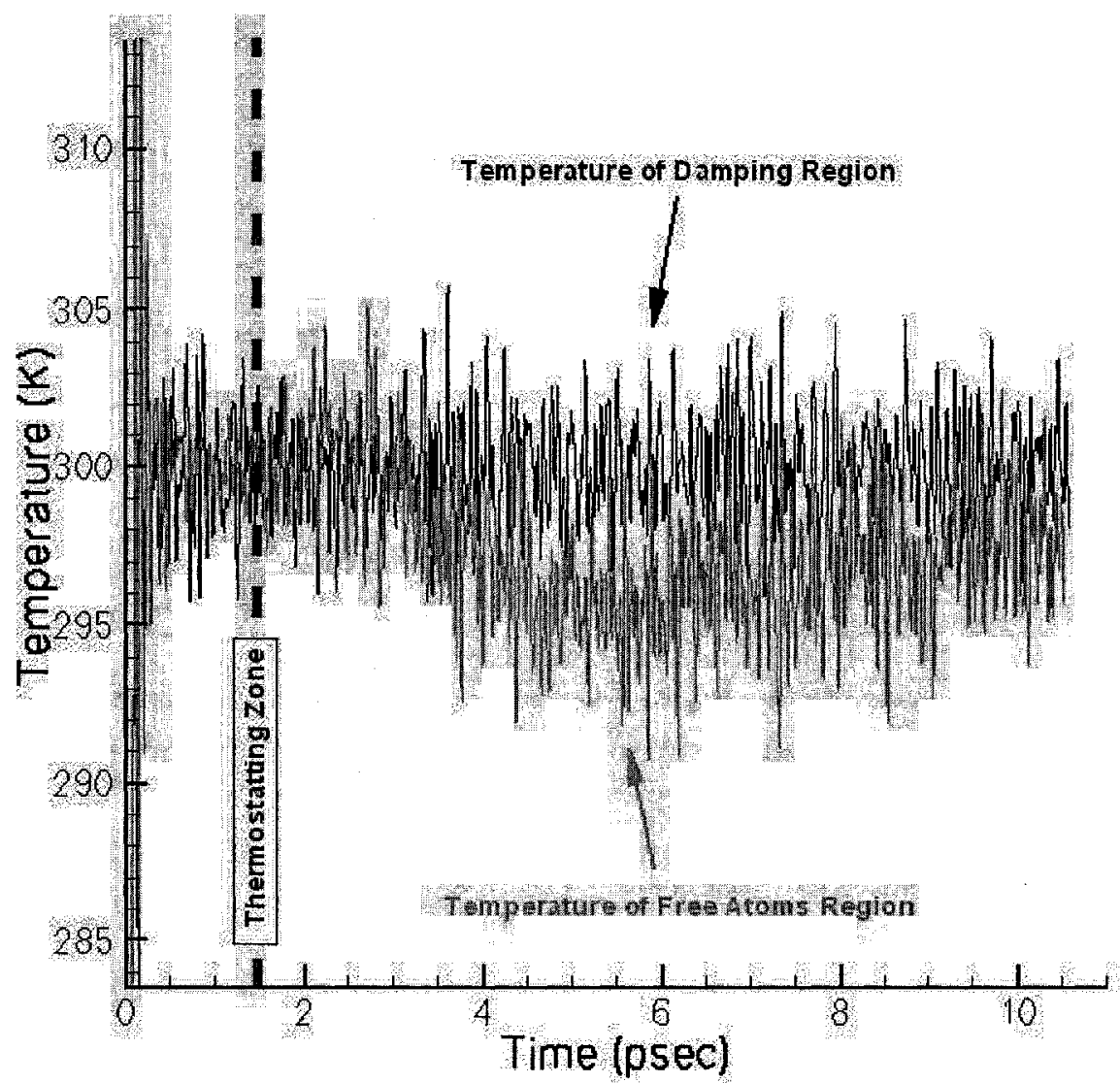

Figure 4.6: Temperature record inside damping and free atoms regions.

make and lose contact with the tip which leads a very noisy load-displacement curves. This highly rigid indenter creates singularities in the force as individual atoms come in contact with the indenter. Based on our test cases, an appropriate value of $C$ in Eq. (4.15) is $1600 \mathrm{~N} / \mathrm{m}^{2}$.

\subsection{Verification Simulations}

Initially, we conduct three simulations to verify the accuracy and limitations of our multiscale method for indentation application. In the first test, a thermal relaxation is performed 
on the model before the tool is advanced towards the substrate. In the beginning, the random velocity components of all atoms are given magnitudes corresponding a proper total energy for an equilibrium system at $300 \mathrm{~K}$ based on the Maxwell-Boltzmann distribution. The Nose-Hoover thermostat is initially applied to both the damping and free-atom regions shown in Fig. 4.4. Then, the thermostat is turned off inside the free atoms region after $1.5 \mathrm{psec}$. The efficiency of the damping band to stabilize the temperature of the undamped atoms, is studied for more than $10 \mathrm{psec}$ or 10000 time steps in the absence of external load. Fig. 4.6 shows the fluctuation of the atomistic region temperature as a function of time during the simulation in both damping and free atoms regions, respectively. The temperature of the region of free atoms remains nearly constant during the simulation when the thermostat is turned off. Snapshot of the displacement distribution of atoms at the end of the test simulation is shown in Fig. 4.7 which shows clearly that the damping band can stabilize the thermal fluctuation of the atoms in the top region.

For the second test simulation, we have performed the indentation simulation to study the effect of the implemented damping band. To examine the efficiency of the NoseHoover thermostat, the velocity of indenter is $300 \mathrm{~m} / \mathrm{s}$ (or $0.3 \mathrm{~nm} / \mathrm{psec}$ ), which can be considered as a high speed indenting process. Fig. 4.8 shows the fluctuation of the atomistic region temperature as a function of time during indentation simulations with and without damping band, respectively. The temperature of the atomistic region increases rapidly during indentation when the damping band is not implemented. This figure clearly shows that the short-length waves, which represent the thermal motion of the atoms at high rate loading, are completely trapped in the atomistic region. This overheating of the atomistic region makes successful continuation of the nanoindentation simulation impossible.

In the third test, the substrate at low temperature, $10 \mathrm{~K}$, is indented to a depth of 5 $\mathrm{nm}$. The simulation time step is $1 \mathrm{psec}$. At each time step the indenter is moved $0.1 \mathrm{~nm}$ down or up for loading or unloading. This results in an indentation rate of $100 \mathrm{~m} / \mathrm{s}$ (or 0.1 $n m / p s e c)$. Although much faster than experimental indentation rates, which are on the 


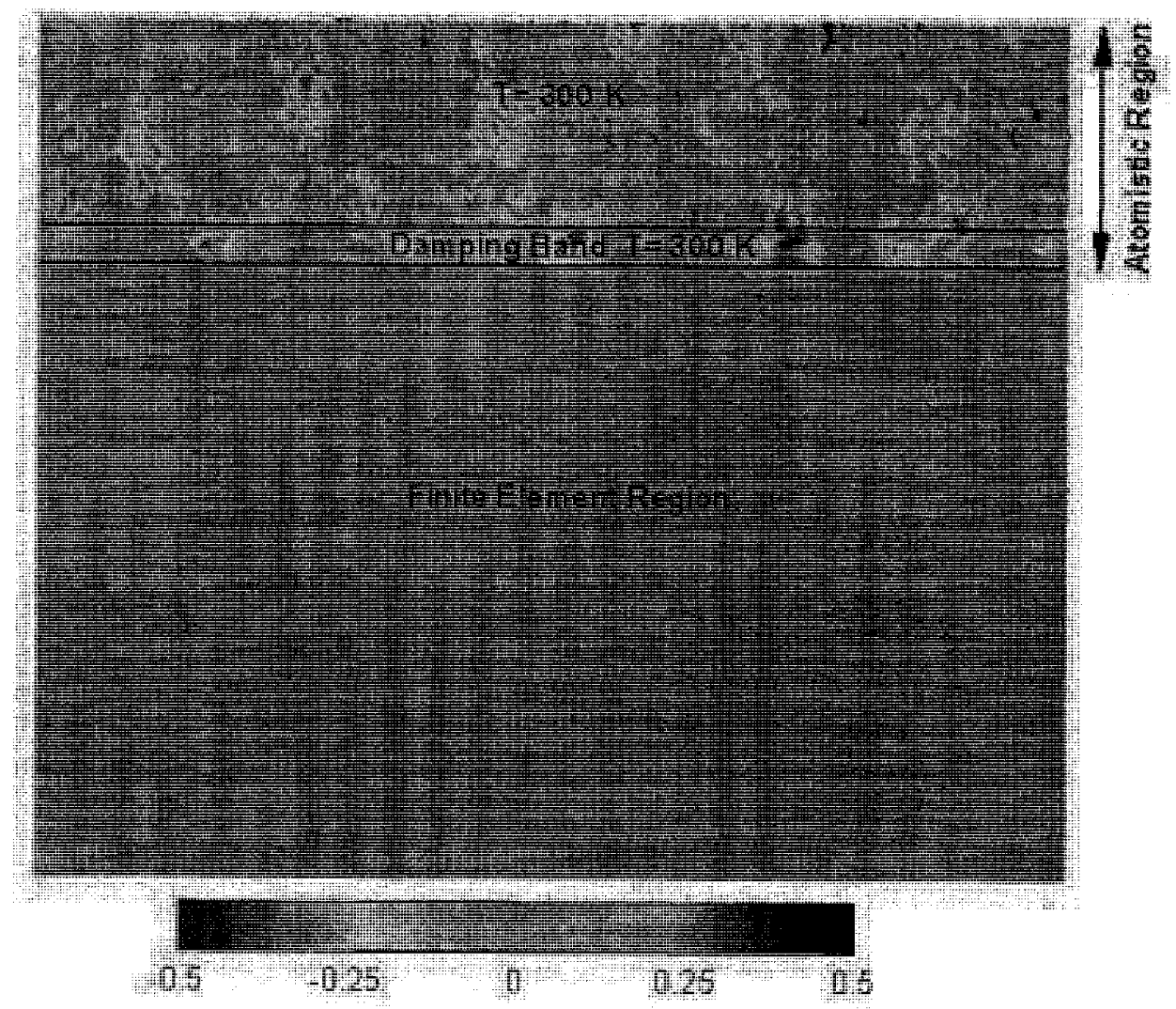

Figure 4.7: Displacement distribution of atoms after thermalization process at desired $300 \mathrm{~K}$. The random velocity components of atoms have magnitude corresponding a proper total energy for an equilibrium harmonic system at $300 \mathrm{~K}$ based on the MaxwellBoltzmann distribution. A damping band of atoms is located between the atom/continuum interface and the undamped atomistic region. Color scale indicates displacement in Angstroms.

order of 1 to $5 \mathrm{~m} / \mathrm{s}$ (or 0.001 to $0.005 \mathrm{~nm} / \mathrm{psec}$ ), the indention rate in our simulations is still approximately 200 times slower than the theoretical dislocation velocity in a triangular aluminum. Fig. 4.9 shows the deformation of the substrate at the maximum loading. The indent depth is about half of the tool radius. Despite the stability of the simulation for such a deep penetration, some elements along the atomistic/continuum interface are highly deformed. Each dislocation during passing the atomistic/continuum interface generates a slip step at the interface. The triangular elements which are close to these steps 


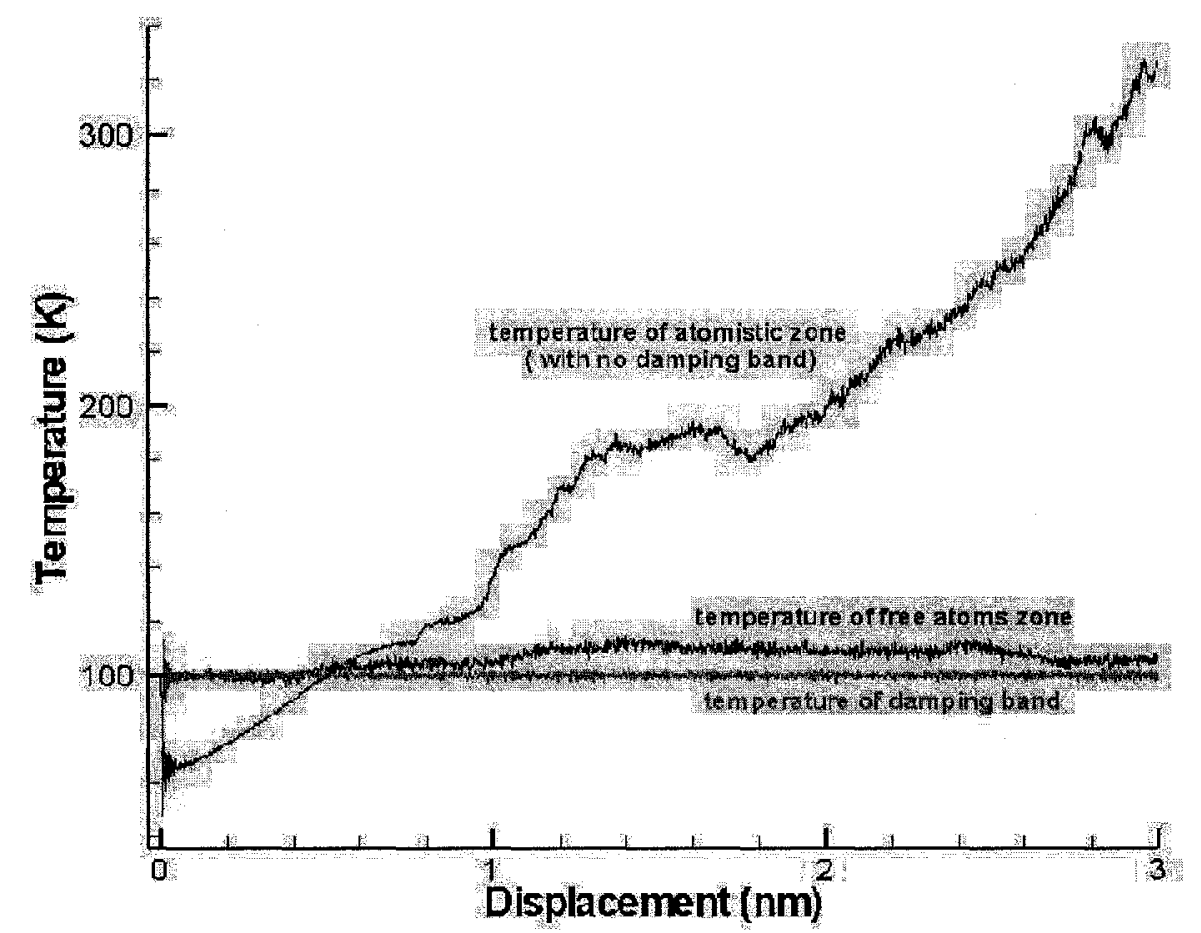

Figure 4.8: Average temperature of free atoms zone increases rapidly when the damping band is inactive during loading process.

and are shown inside the circle in Fig. 4.9 are heavily distorted. The strain field in those elements is not elastic anymore. This test shows that the error in the continuum strain field calculation increases in a deep indention because of using linear finite elements as the supporting boundaries around the atomistic region.

The load-displacement curve of the test simulation is plotted against the quasi-static load-displacement curve reported in Ref. [71] in Fig. 4.10. The data of the dynamic simulation appears quite noisy compared to the force-displacement curve obtained from the static $C A D D$ simulation. To generate good data from the noise a smoothing technique is applied. The smoothing technique shifts the value of the force at the data point towards an average of the values at its neighboring data points in each pass of smoothing. A 


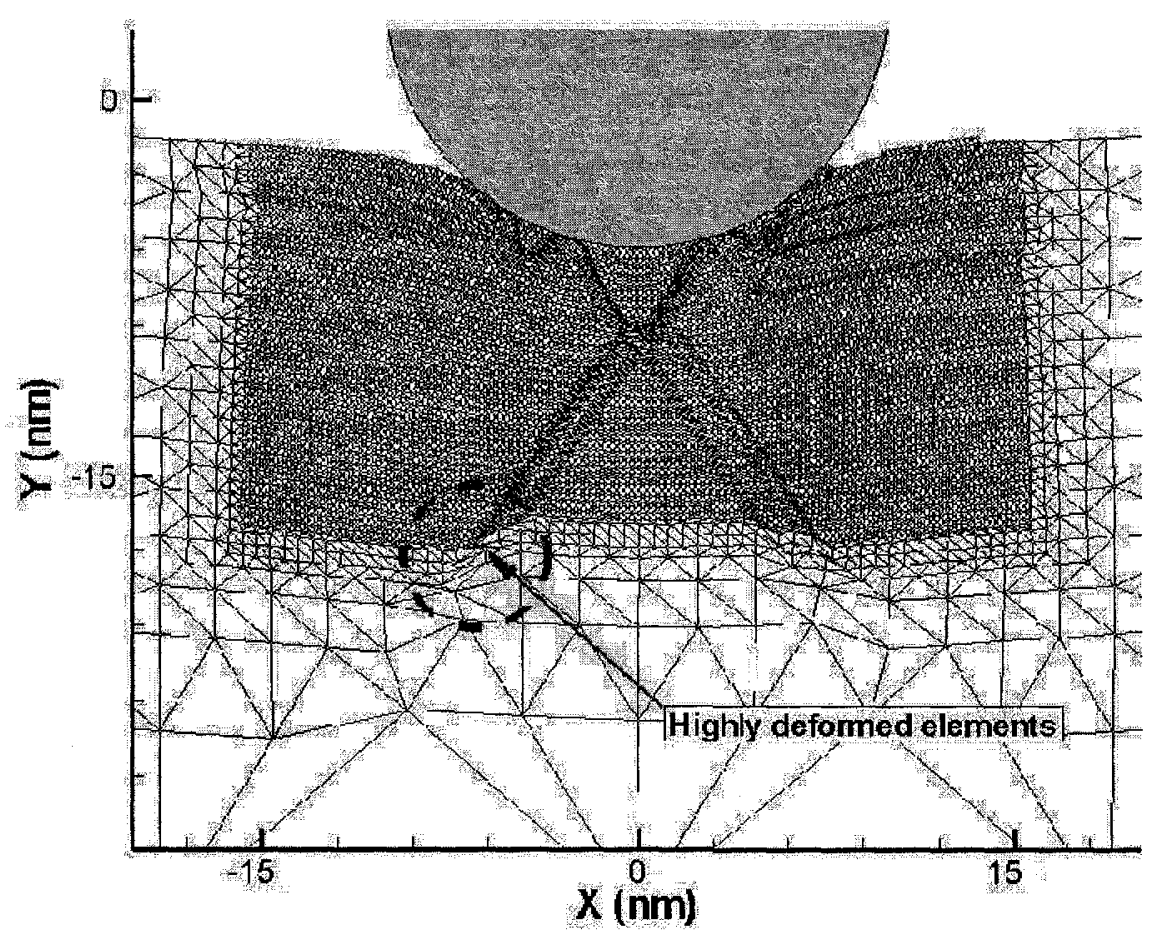

Figure 4.9: Deformation of substrate during a deep penetration of an indentor.

total of 1500 passes of smoothing was applied to remove the noise before plotting Fig. 4.10. Critical points on the curves are labelled by roman numerals. From the start of the loading to point $I_{d}$ or $I_{s}$, the response is completely elastic for both curves which lies in the Hertzian regime. This section of the curves represents a linear-elastic relation between the indenter and the surface, i.e. no plasticity and no adhesion.

In Fig. 4.10, the step from $I_{d}$ (or $I_{s}$ ) to $I I_{d}$ (or $I I_{s}$ ) corresponds to the first nucleation of dislocations. As it can be seen, the dynamics method predicts the first dislocation nucleation at smaller depth of indentation in comparison to the static simulation. This makes sense since thermal fluctuations will precipitate earlier activation of nucleation. 


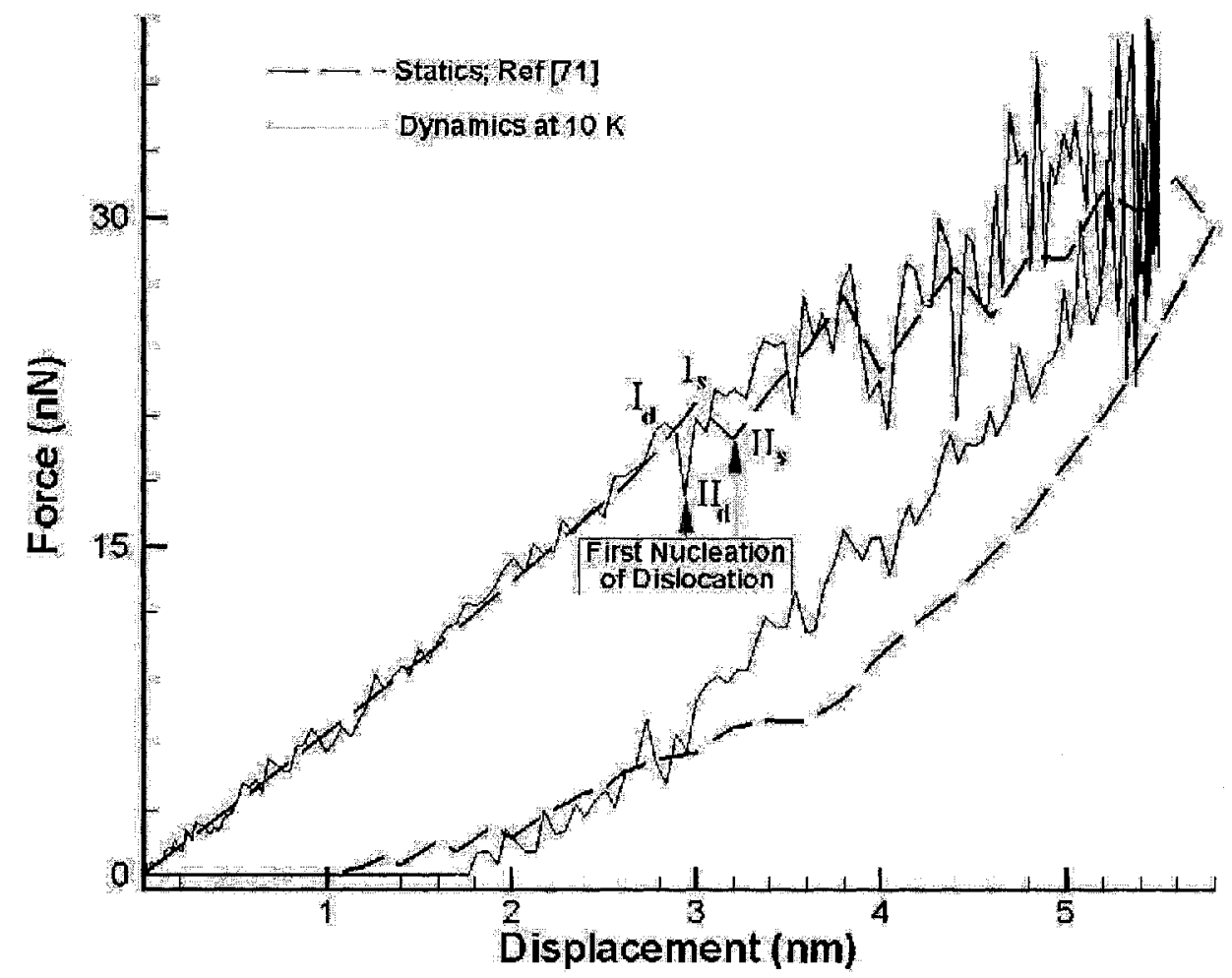

Figure 4.10: Load-displacement curves from static and dynamic $C A D D$.

\subsection{Results and Discussion}

In this section, we perform a systematic study to investigate qualitatively, various factors that dominate nanoindentation behaviors. Emphases are placed on the characteristics of load-displacement curve and dislocation nucleation events as a function of indentation speed and substrate temperature.

First, the substrate shown in Fig. 4.3, is indented under a constant rate of $100 \mathrm{~m} / \mathrm{s}$ (or $0.1 \mathrm{~nm} / \mathrm{psec}$ ) at $200 \mathrm{~K}$. The states of deformation at the peak load (4 $\mathrm{nm}$ depth of penetration) and after unloading are shown in Figs. 4.11(a) and (b). The discrete dislocations are plotted as circles. The figures show that a number of dislocations have moved out of the 


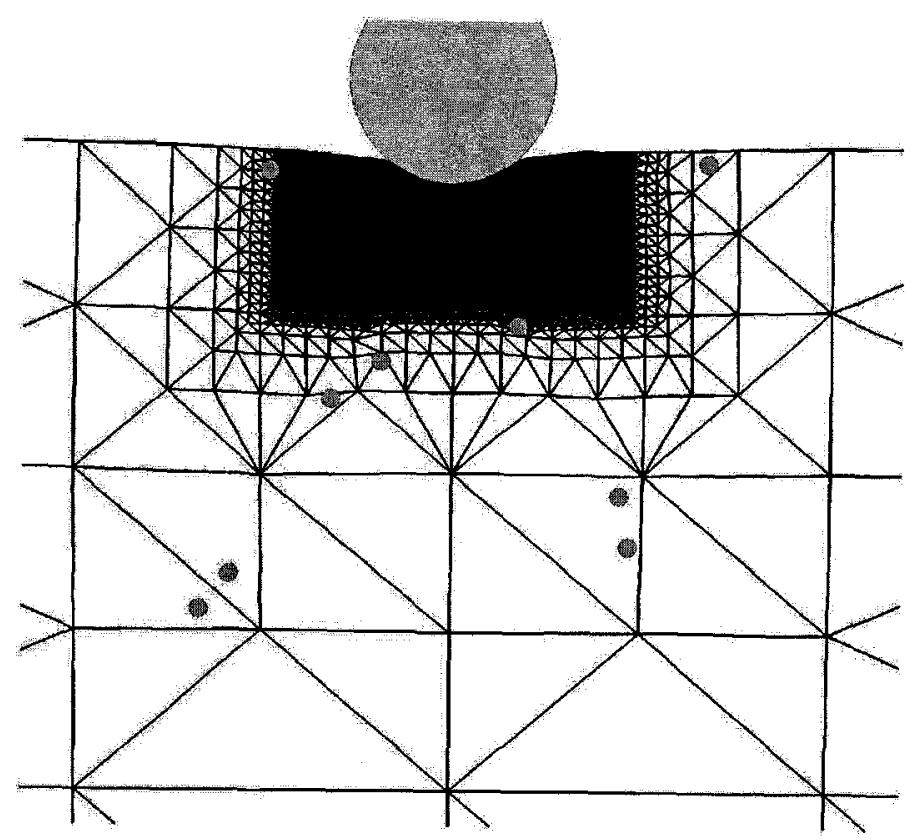

(a)

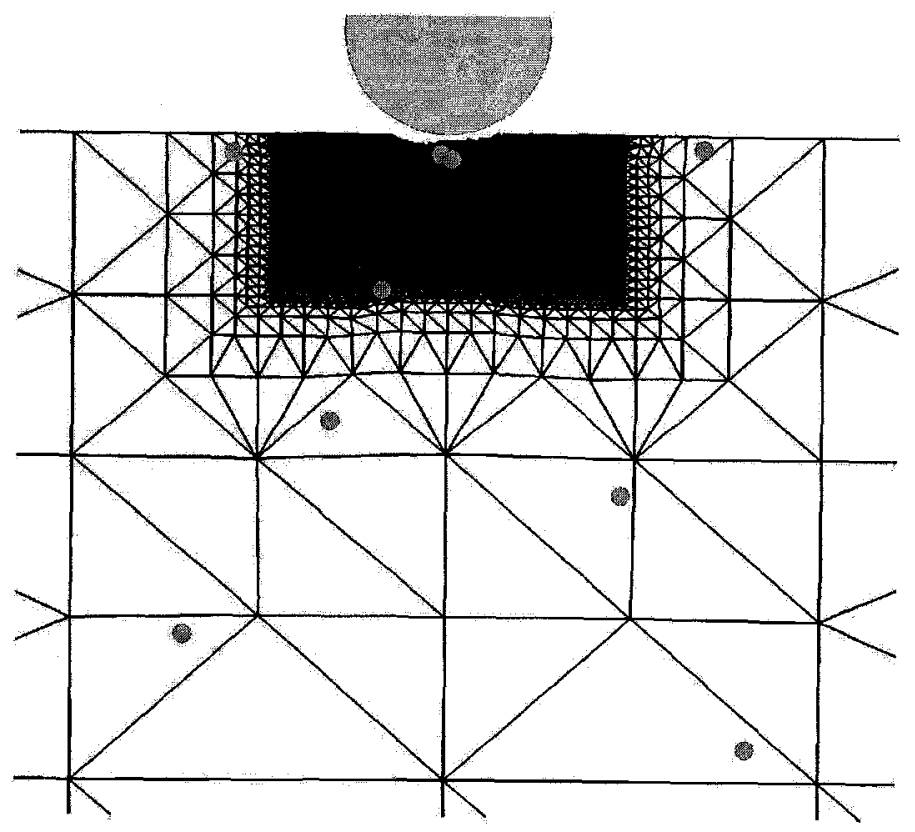

(b)

Figure 4.11: Configuration of positions of the discrete dislocations and the atomic region at maximum load (a), and after unloading (b). 
bottom of the atomistic region into continuum region. Due to the large deformation state in the atomistics, however, these dislocations are not all on the same slip plane, which will have consequences for the unloading behavior. In addition, several dislocation have moved out of the left and right sides of the atomistic region due to the dissociation mechanism. Upon unloading from the peak load, the continuum dislocations glide back toward the indenter as shown in Fig. 4.11(b).

The simulations show the same dislocation nucleation processes that has been observed at zero temperature by Miller et al. [71]. A series of snapshots of nucleation and propagation of dislocations inside the atomistic region during loading process are depicted in Fig. 4.12(a) to (f). Various research groups have used different dislocation visualization methods $[85,86,87,88]$. As it can be seen in Fig. 4.12, we have used a finite element mesh joining the atoms, rather than atoms themselves, in our $2 D$ simulations. This is helpful for visualization of dislocations activity in atomistic region, as the mesh is defined in the reference, perfect lattice configuration. The first edge dipole pair nucleates inside the atomistic region as shown in Fig. 4.12(a). Upon nucleation, this dislocation dipole splits immediately and moves along the slip planes located at $+30^{\circ}$ from vertical as shown in Fig. 4.12(b). One of these dislocations moves up and hits the surface of the indenter, where it is emitted back into the crystal along a new slip plane as shown in Fig. 4.12(c). The other dislocation travels down and outs the bottom of the atomistic region, where it is converted to discrete dislocations that eventually come to rest near lower boundary of model (Fig. 4.12(d)). As can be seen in Fig. 4.12(e), a second dipole gets nucleated a short time (about $3 \mathrm{ps)}$ ) after the first nucleation at the same location beneath the indenter. For the second dipole, one member of dipole pair travels up to the surface along the slip plane oriented at $-30^{\circ}$ to the vertical. Fig. 4.12(f) shows that the reflected defects from the indenter surface ultimately dissociate onto horizontal slip planes and move to the left and right of the atomistic region. This dissociation mechanism is, of course, a feature that is specific to the two-dimensional triangular lattice, but it can be viewed as analogous to 


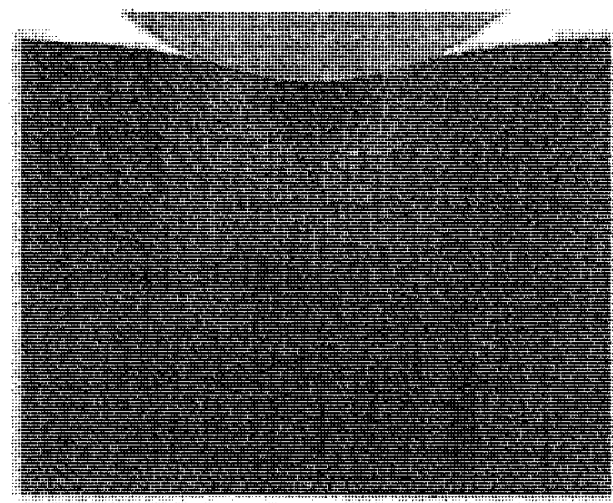

1)

(a)

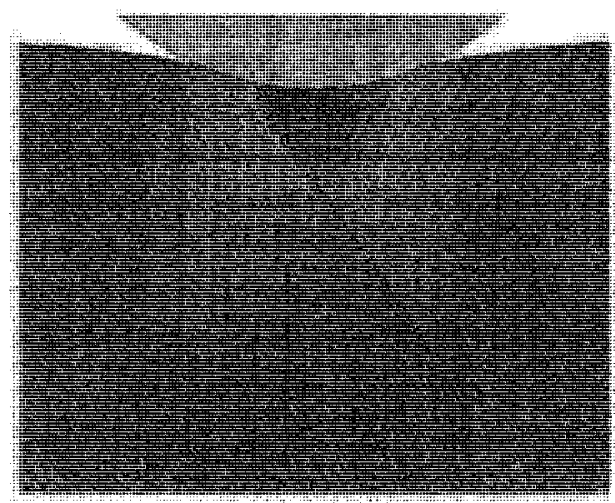

a: 3 ती

(c)

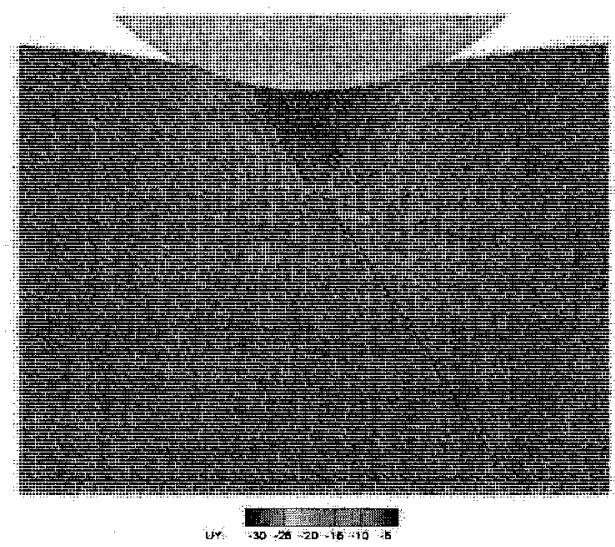

(e)

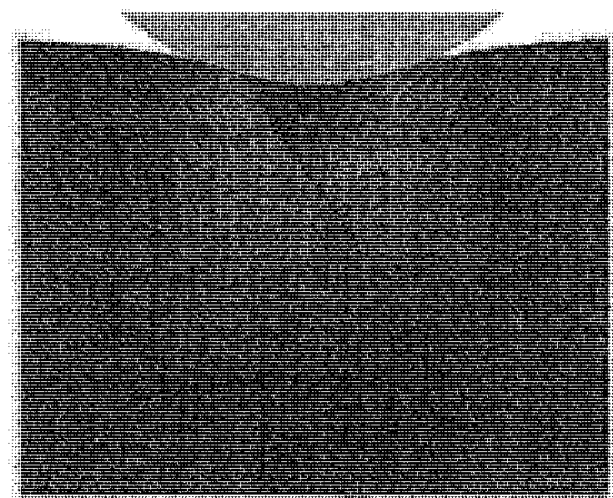

(1)

(b)

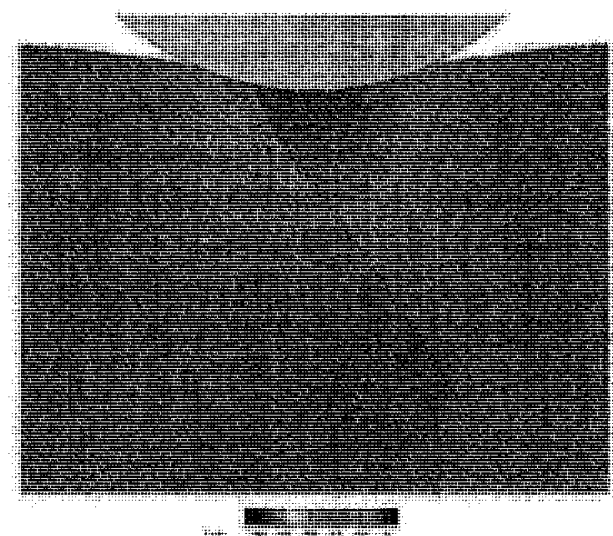

(ii)

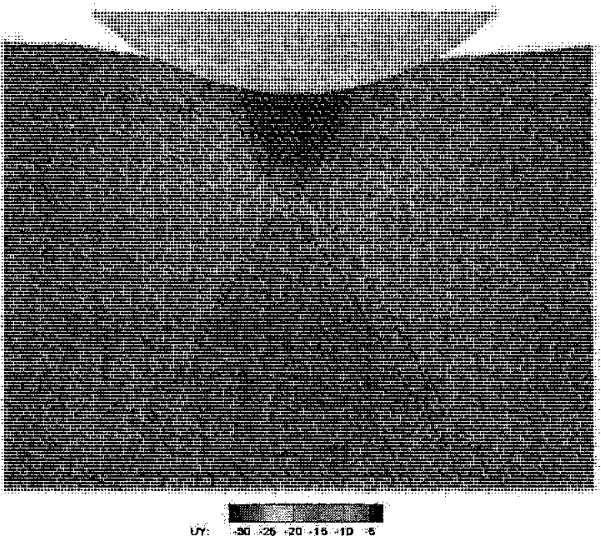

(f)

Figure 4.12: Snapshots of the configuration of the atomistic region during initiation and propagation of dislocations 
cross-slip and other dislocation reactions that occur in three-dimensional simulations. For example, Saraev and Miller $[89,90]$ observed dislocations moving laterally from beneath a three-dimensional indenter.

To study the variation of temperature during indentation and its relation to dislocation nucleation, several simulations are conducted. Fig. 4.13 shows the variation of average temperature inside the free atoms region for four different initial temperatures of $100 \mathrm{~K}$, $200 \mathrm{~K}, 300 \mathrm{~K}$, and $400 \mathrm{~K}$, respectively. The indentation loading and unloading speed is $0.1 \mathrm{~nm} / \mathrm{psec}$ (or $100 \mathrm{~m} / \mathrm{s}$ ). As it can be seen, the temperature increased in the atomistic region during the loading process. This rise reached a maximum value when the indenter reached its maximum depth of penetration. The temperature decreased during the unloading process. In this region the temperature fluctuated with an amplitude proportional to the initial set temperature. As it can be seen, the average temperature of the damping band region remains constant for all the simulations.

Three typical load-displacement curves are plotted in Fig. 4.14. The thermal noise is suppressed by the averaging method. As seen in the figure, the onset of plasticity or dislocation nucleation is affected by the temperature. Increasing temperature caused the nucleation of dislocation to happen earlier. This phenomenon was recently observed by using the finite temperature quasicontinuum [43]. The load-displacement curve is essentially reversible as long as the indentation depth is less than the critical value corresponding to the first dislocation nucleation that is indicated in Fig. 4.14. The variation of this critical value versus temperature is plotted in Fig. 4.15. As it is shown, a quadratic equation fit the data as

$$
d_{c}=1 E^{-6} T^{2}-6.5 E^{-3} T+3.856
$$

where $d_{c}$ is critical depth in $n m$ and $T$ is temperature in $K$. The coefficient of determination $\left(R^{2}\right)$ for the above quadratic equation is 0.9986 . So the quadratic is an excellent fit to the data.

There is an interesting compatibility between the major humps shown in Fig. 4.13 


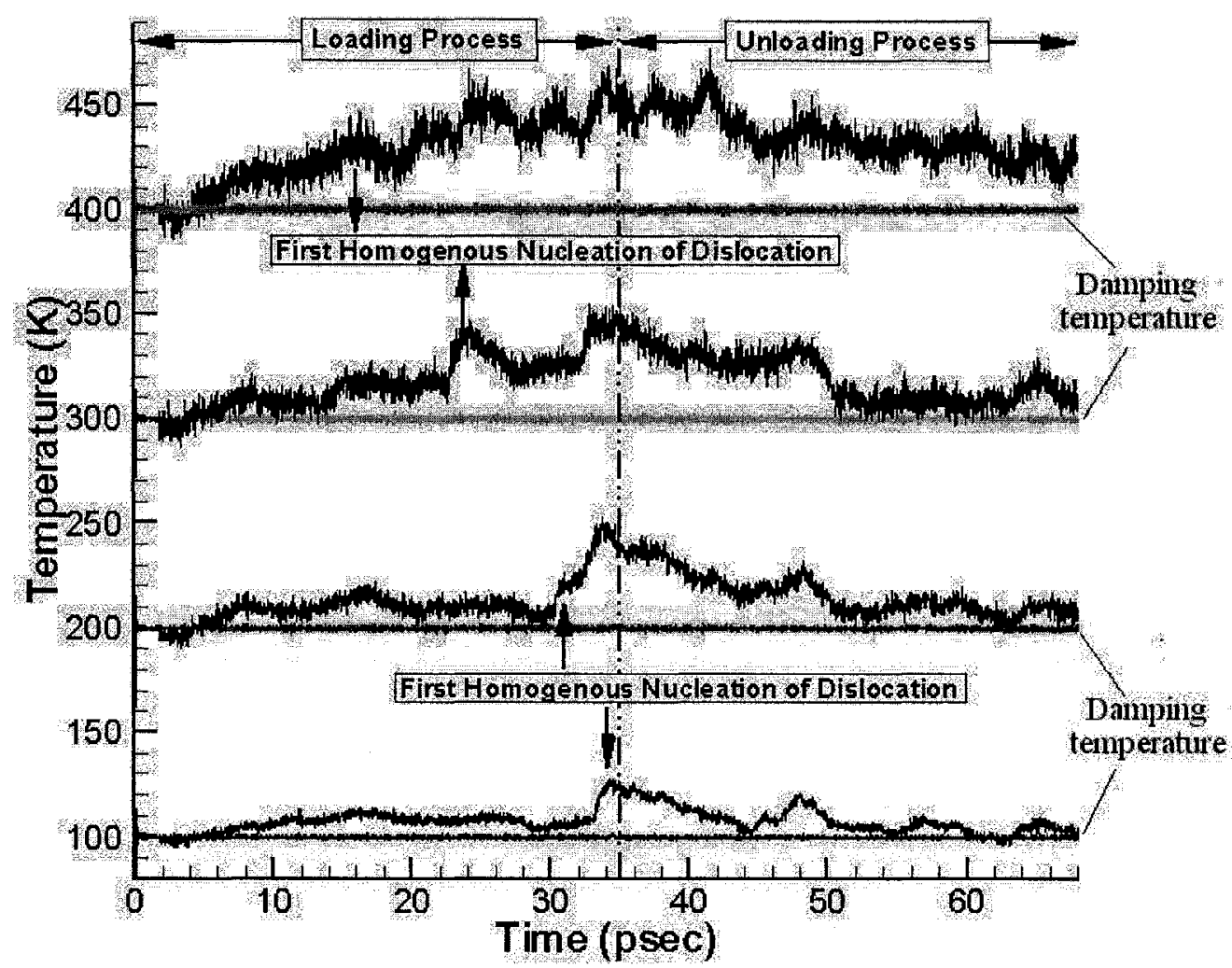

Figure 4.13: Average temperature of damping band and free atoms region during indentation a substrate at four different initial temperatures $100 \mathrm{~K}, 200 \mathrm{~K}, 300 \mathrm{~K}$ and $400 \mathrm{~K}$. 


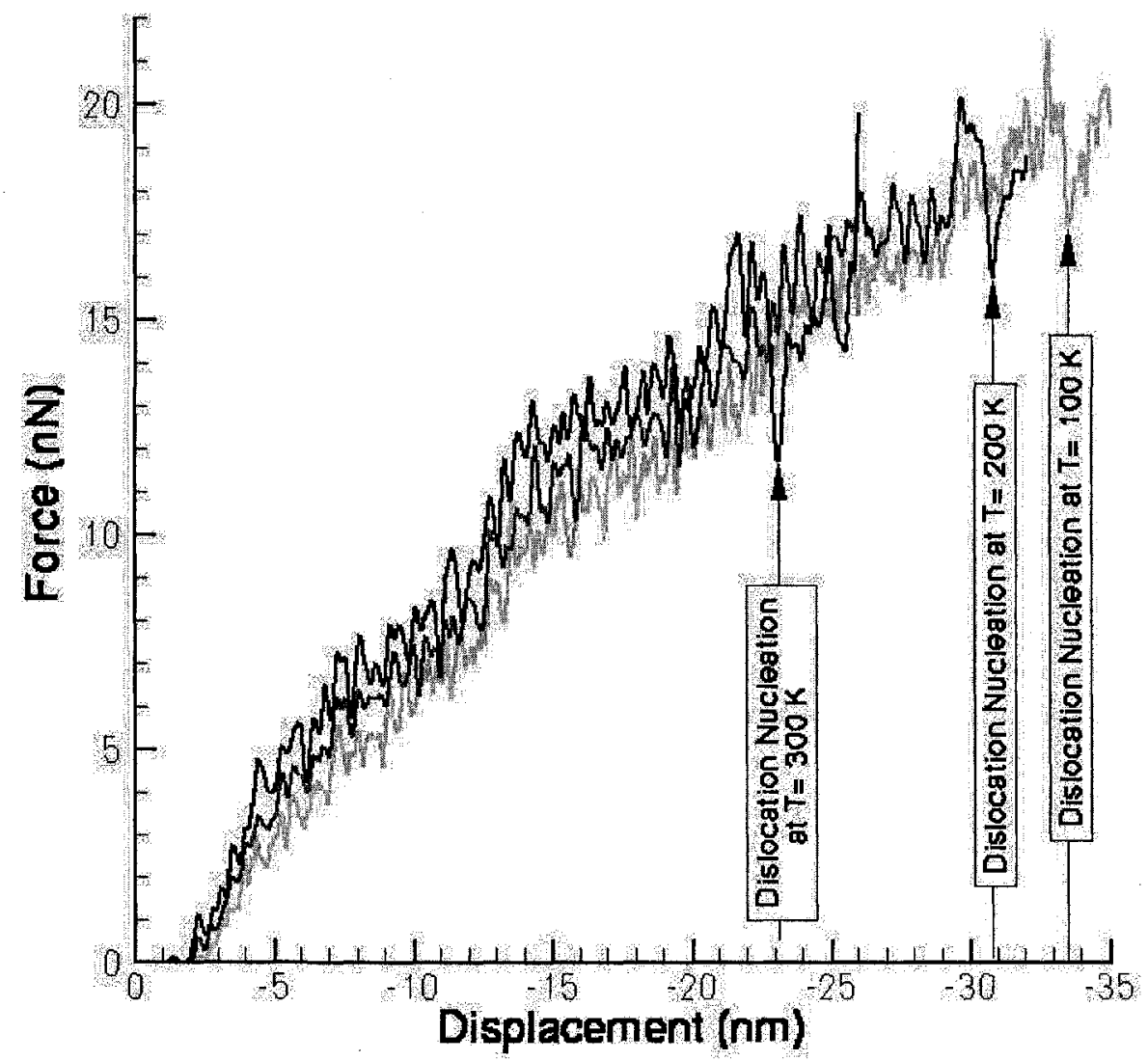

Figure 4.14: Initial homogeneous nucleation of dislocation beneath the indenter at three different temperatures, $100 \mathrm{~K}, 200 \mathrm{~K}$ and $300 \mathrm{~K}$, respectively.

and the onsets of plasticity events shown in Fig. 4.14. The temperature of the free atoms region increases noticably during generation of dislocations. There is a clear one-to-one correlation between the number of dislocation generation events during indentation process and the number of temperature humps as indicated in Fig. 4.13.

Fig. 4.16 shows the load-displacement curves for one complete loading/unloading cycle at three different initial set temperatures, $10 \mathrm{~K}, 100 \mathrm{~K}$ and $300 \mathrm{~K}$. The depth of penetration is $3.8 \mathrm{~nm}$ for the simulations. A visible increase of the hysteresis area happened when the simulation temperature increased from $10 \mathrm{~K}$ to $300 \mathrm{~K}$. This increase happens be- 


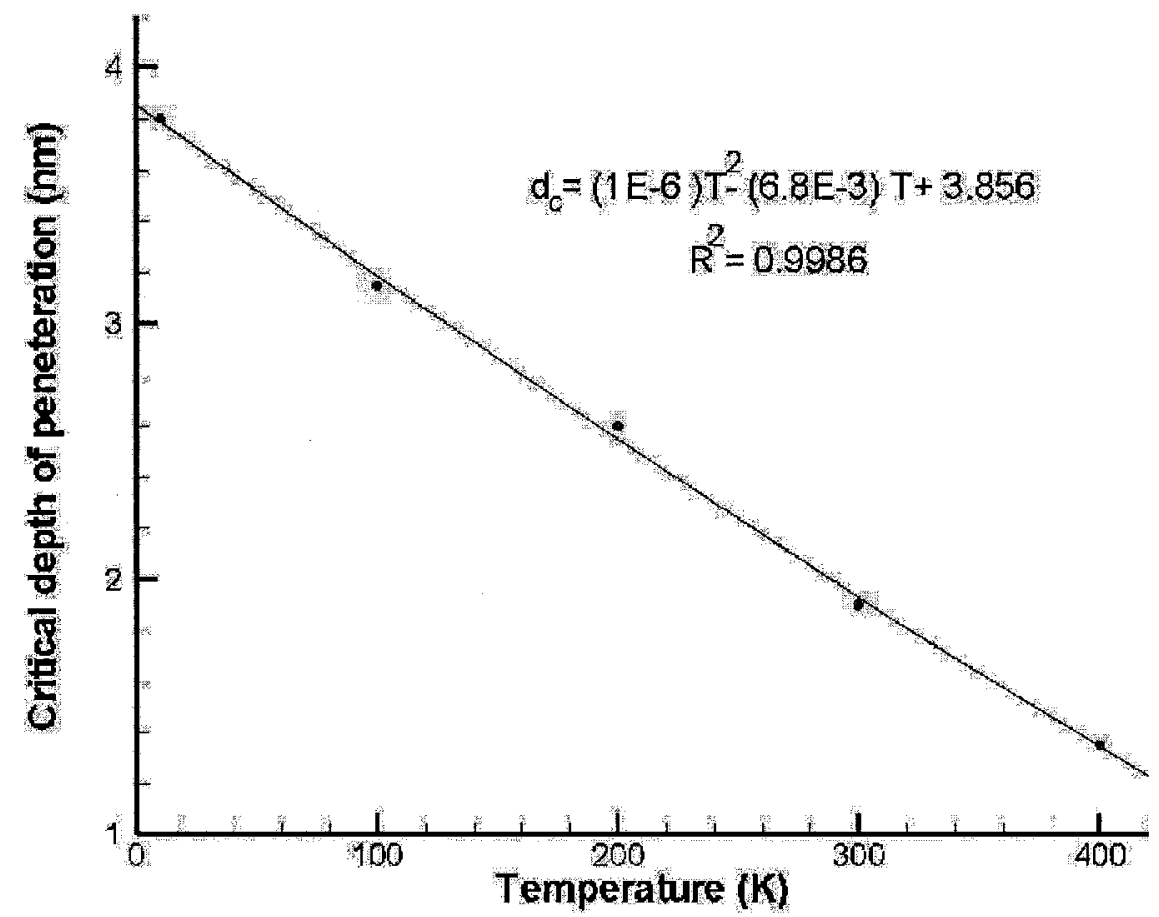

Figure 4.15: Variation of critical depth for dislocation nucleation with increasing temperature.

cause of the accumulation of more permanent deformation in the higher temperature state $\left(\delta_{p}^{10}<\delta_{p}^{100}<\delta_{p}^{300}\right)$ which is related to the increase of the number of homogenous dislocations by increasing the substrate temperature. As the figure shows, there is less plastic recovery at higher temperature. Fig. 4.16 also indicates, for the maximum depth of indentation, the load of indentation decreases as the temperature increases.

Fig. 4.17 shows the first nucleation site of the homogenous dislocation beneath the indenter for three different temperature, $10 \mathrm{~K}, 100 \mathrm{~K}$ and $300 \mathrm{~K}$, respectively. The nucleation site in each simulation, is located at a depth of approximately $0.86 a$, where $a$ is the half-width of the contact area between the substrate and the indenter. This is consistent with the depth of the maximum resolved shear stresses predicted by Hertzian theory $(0.78$ a), but in fact the Hertzian maximum stresses occur on planes different from those on 


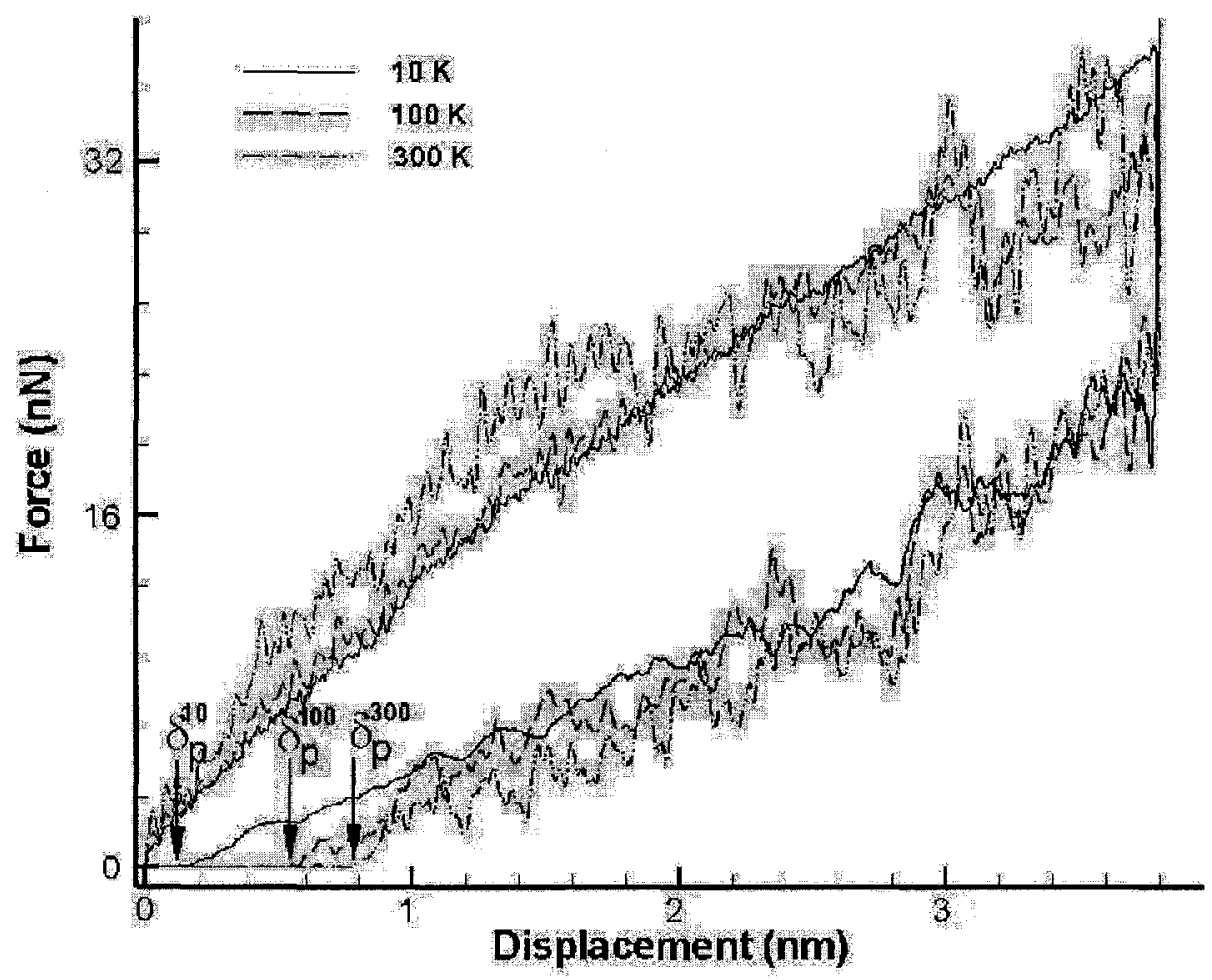

Figure 4.16: Nanoindentation load-displacement curves for three different temperatures, $10 K, 100 K$ and $300 K$, respectively.

which the defects first form. Defining the maximum resolved shear stress on any of the three slip systems $\left(0,+\frac{\pi}{6}\right.$ and $-\frac{\pi}{6}$ with respect to the vertical line $)$ as

$$
\tau_{r}^{\max }=\max \left(\tau_{r}^{1}, \tau_{r}^{2}, \tau_{r}^{3}\right)
$$

where $\tau_{r}^{i}$ is the resolved shear stress on the $i-t$ th slip system, Fig. 4.18 shows $3 D$ contours of $\tau_{r}^{\max }$, just prior to defect nucleation, as computed from the atomistic stresses (virial stresses) [91]. Clearly, the maximum stresses (the darkest contours) occur a distance from the actual nucleation site, indicating that the maximum resolved shear stress is not the correct parameter to predict nucleation. The fact that the maximum resolved shear stress on the possible slip planes is likely not to lie on the indenter axis is firstly pointed out 
by Kelchner et al. [92]. A number of researchers have recently proposed alternatives to the maximum stress criterion for dislocation nucleation based on similar observation [93, 91, 94]. 


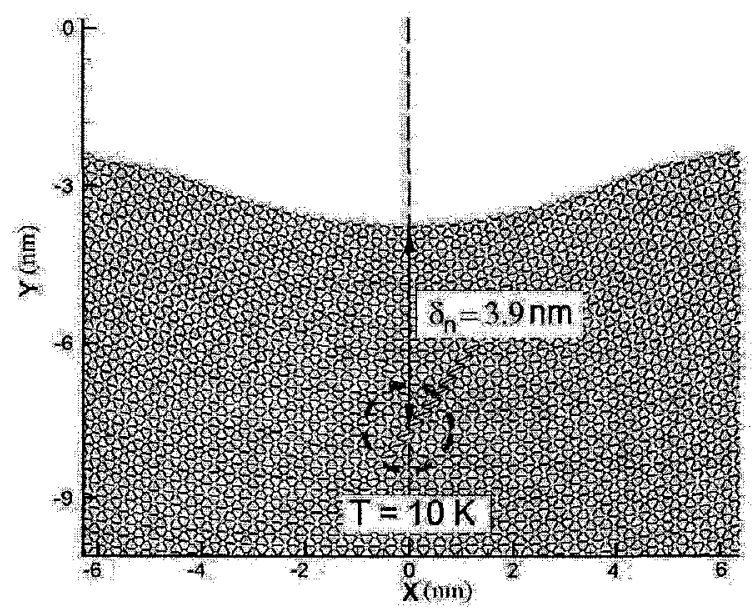

(a)

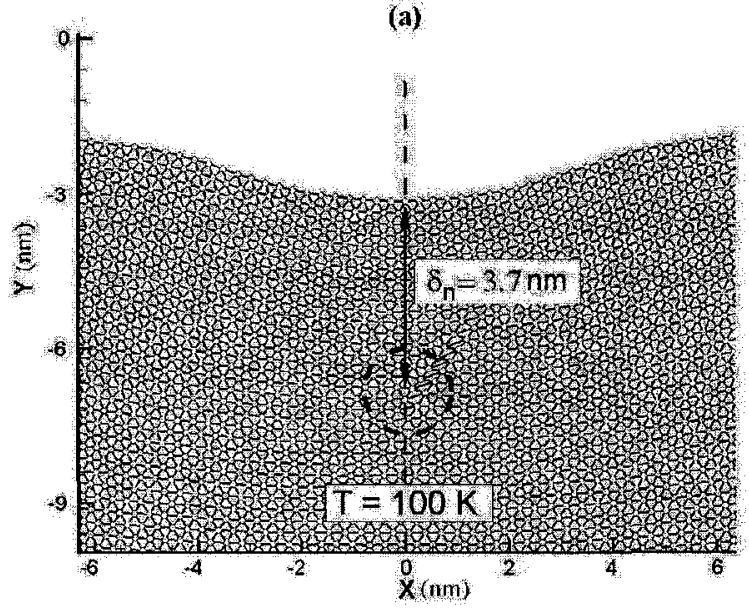

(b)

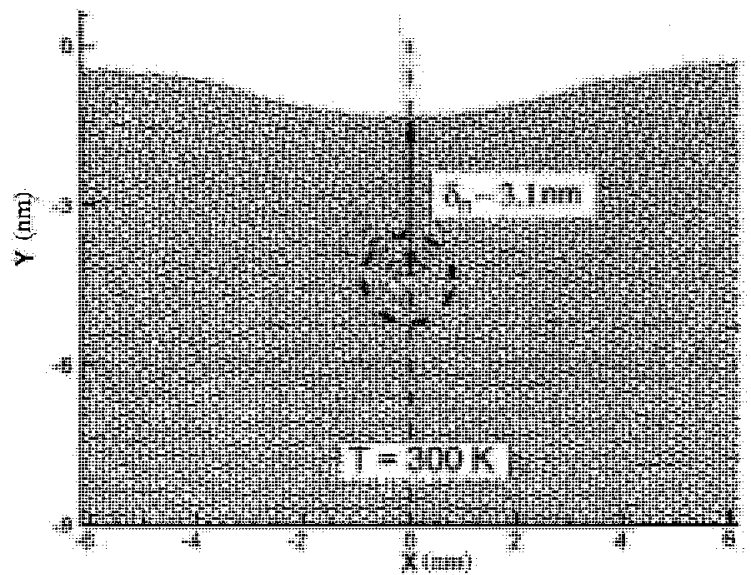

(c)

Figure 4.17: Initial homogenous nucleation of edge dislocation dipoles at a depth of approximately $0.86 a$, where a is the half width of the contact area between the substrate and indenter, for three different temperature, $10 \mathrm{~K}, 100 \mathrm{~K}$ and $300 \mathrm{~K}$, respectively. 


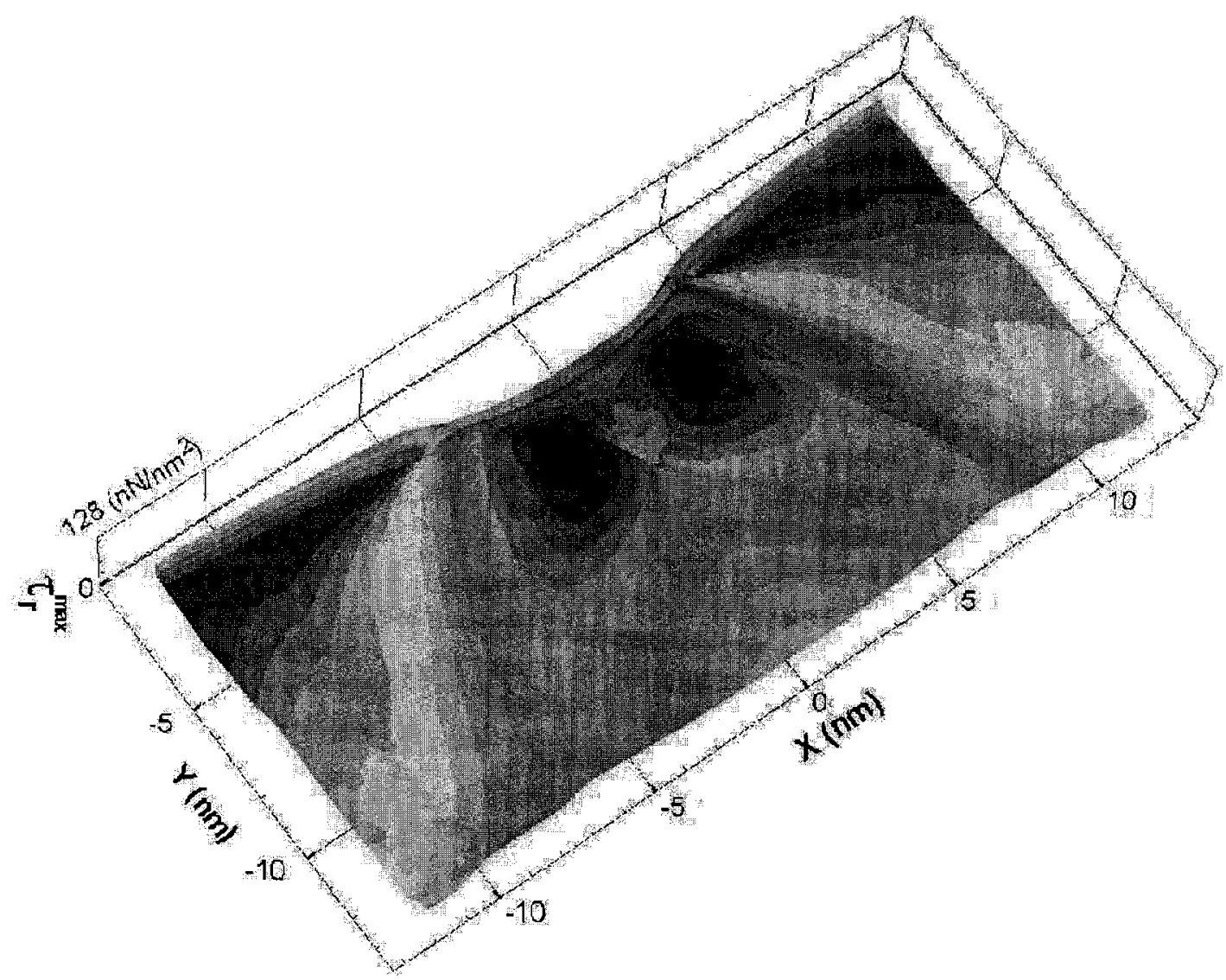

Figure 4.18: Maximum critical resolved shear stress prior to dislocation nucleation. The largest values of $\tau_{r}^{\max }$ are not located at the site of nucleation. 

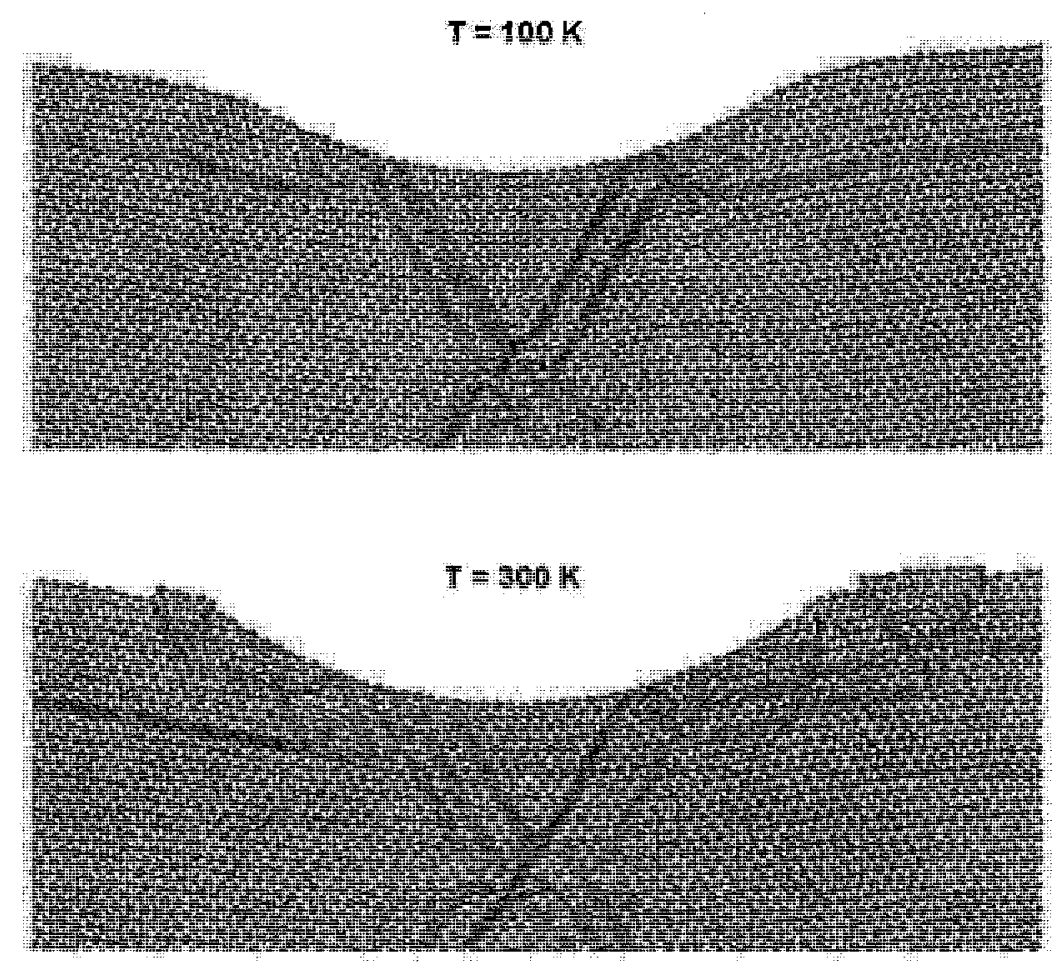

Figure 4.19: Profiles of the atomistic surfaces after $4.2 \mathrm{~nm}$ indentation at two different temperatures, $100 \mathrm{~K}$ and $300 \mathrm{~K}$. The mesh is drawn between the atoms in the atomistic region for visualization purposes and the deformed elements clearly highlight the slip planes in the wake of the dislocations

Fig. 4.19 shows the profile of the deformed atomistic surface under the indenter after $4.3 \mathrm{~nm}$ depth of indentation at two different temperatures, $100 \mathrm{~K}$ and $300 \mathrm{~K}$. It can be seen that the number of generated dislocations beneath the indenter was noticeably increased by increasing the temperature of the atomistic region from 100 to $300 \mathrm{~K}$. There is clearly permanent deformation and flow of material, and atomic scale analogues of several largerscale indentation features are evident. Most notably, Fig. 4.19 shows a pile-up of material on either side of the indenter that stands higher than the original undamaged surface at $300 \mathrm{~K}$. Analysis of the sequence of events forming this pile-up during the deformation reveals that the dislocation dissociation mechanism described earlier is key to the pileup 
process. This suggests that in a $3 D$ nanoindentation, pile-up requires a significant degree of cross-slip to take place during the deformation.

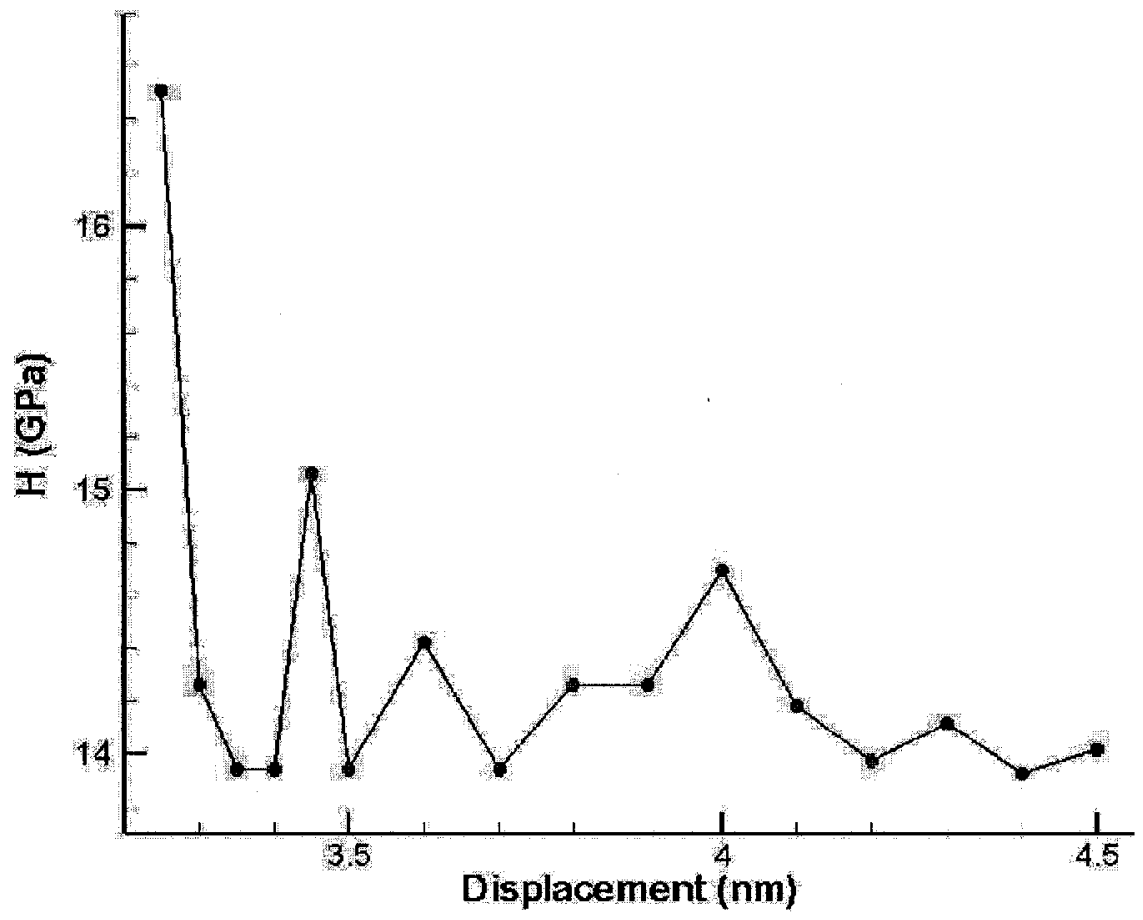

Figure 4.20: Variation of hardness "load divided by contact area" with increasing indentation depth at $10 \mathrm{~K}$

In Fig. 4.20, the hardness obtained between the critical point and maximum load is illustrated. After this critical point, dislocation dipole pairs continue to nucleate and move out of the atomistic region. All the nucleation events occur almost at the same location beneath the indenter. During this part of the loading process, the hardness $H$ (load divided by the contact area) can be calculated in each time step. The discrete nucleation events between the critical point and maximum load, lead to dramatic variations in hardness, but in general the hardness decreases with increasing depth of indentation. This phenomenon has been observed by Miller et al. [71]. This phenomenon is consistent with a number of 
theoretical models that attempt to explain the indentation size effect. However, we note that the nucleated dislocations during simulation hit the indenter surface and are reflected back. This leaves a residual dislocation at the surface whose effect is to increase the surface area of the crystal.

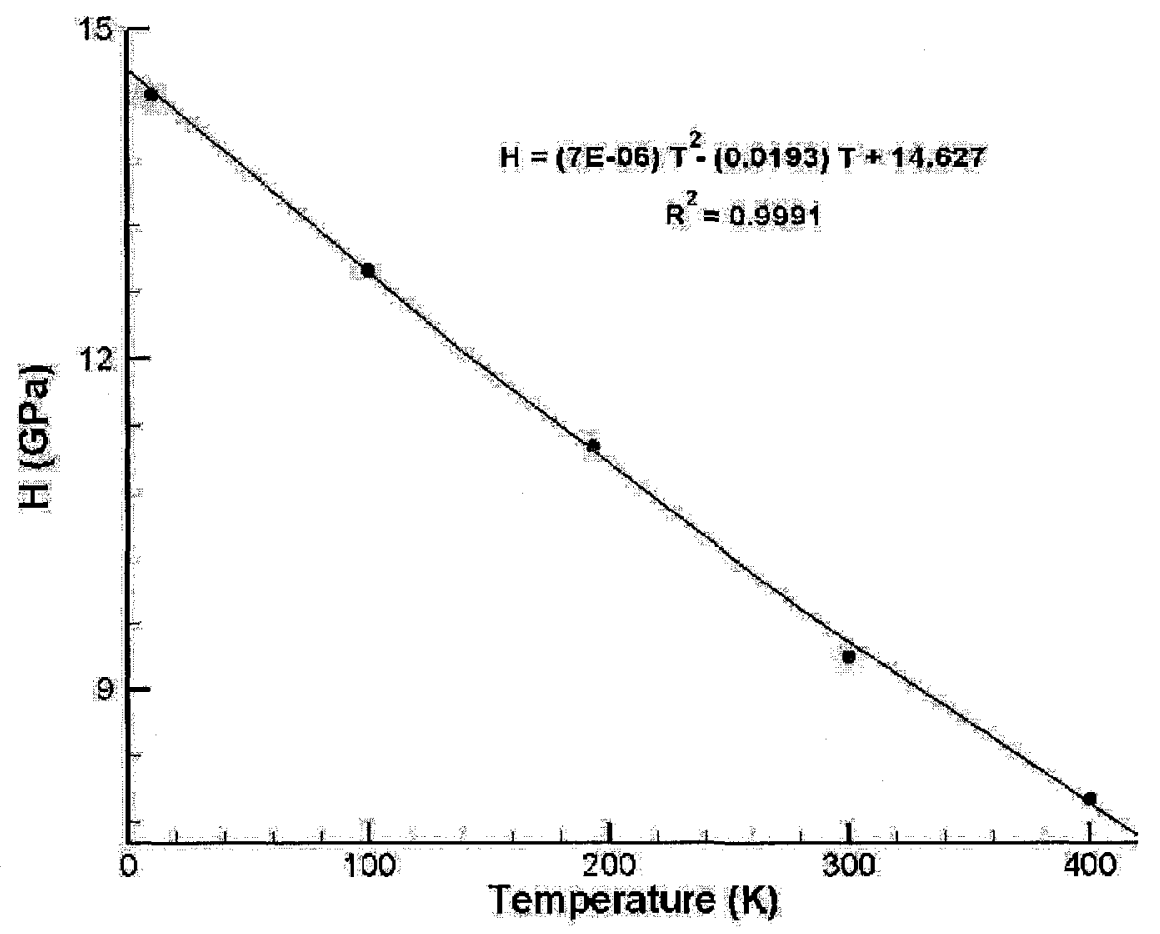

Figure 4.21: Variation of hardness at maximum load versus temperature of the atomistic region

The relationship between the temperature and hardness of the substrate is shown in Fig. 4.21. The hardness is calculated using central averaging around the maximum load. A quadratic fit of the data to the form $H=\left(7 E^{-6}\right) T^{2}-(0.0193) T+14.627$ is shown in the figure, and provides reasonably excellent agreement $\left(R^{2}=0.9991\right)$. This behavior is consistent with macro behavior. From the micro-scale viewpoint, the substrate interatomic bonding energy decreases as interatomic bonding distance increases by temperature, leading to the hardness decrease. This finds confirmation with the experimental studies in the 
literature. In other words, the sample material softens at higher temperature.

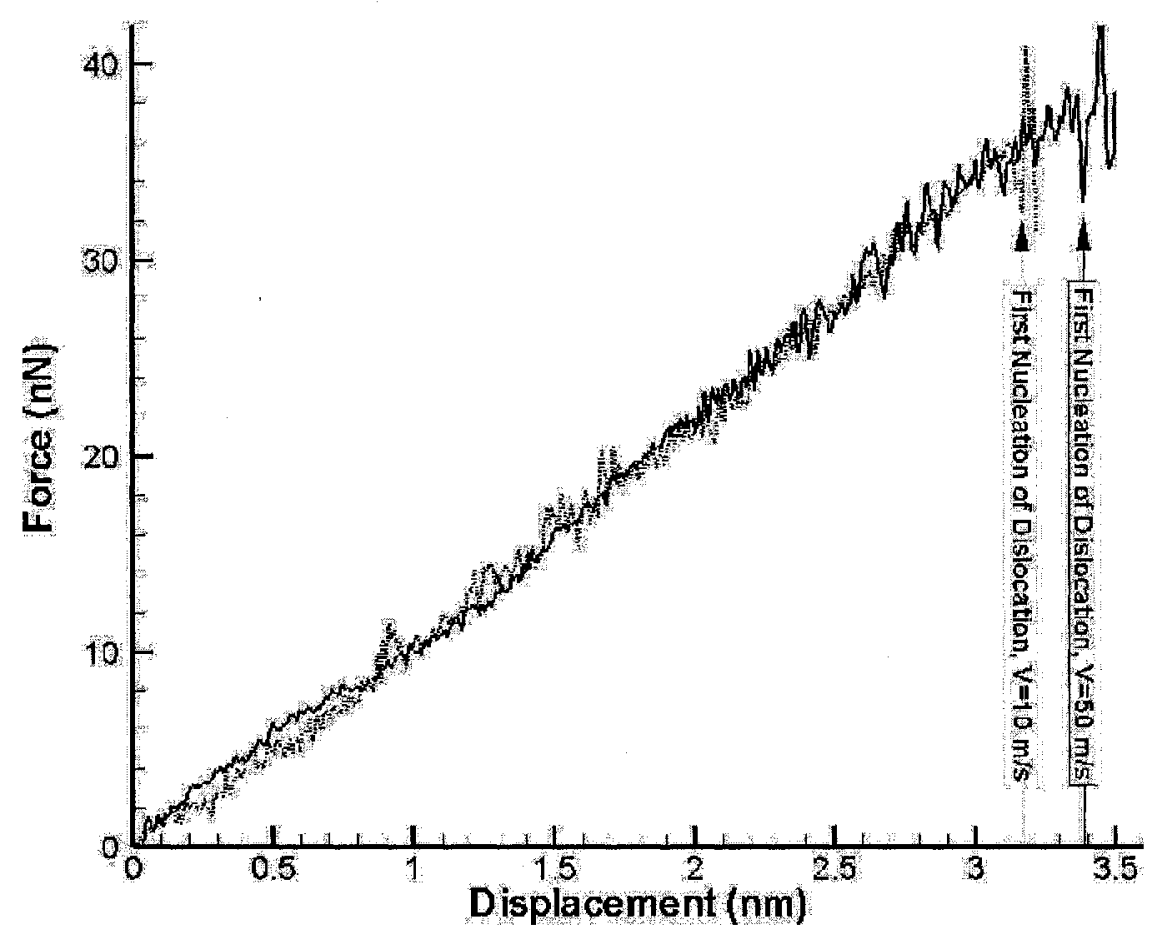

Figure 4.22: Load-displacement curves for two different velocities of indentation at $10 \mathrm{~K}$.

In order to study the dependence of the elastic-to-plastic transformation on indentation speed, simulations are carried out for different indentation velocities at $10 \mathrm{~K}$. Two typical loading curves for two different velocities are plotted in Fig. 4.22. As is seen in the figure, we note that the critical depths corresponding to the force-displacement curves are not essentially the same for all velocities, thus indicating a rather noticeable dependence of the critical depth on velocity. Increasing velocity makes the nucleation of dislocation happen later.

By increasing the indentation velocity, the dislocation nucleation can not be clearly observed. In the high rate indenting simulations, the deformation of the substrate under the indenter is highly localized and the stored elastic energy is not sufficient to cause 
homogenous dislocation nucleation. By increasing the indenting rate or velocity more than $100 \mathrm{~m} / \mathrm{s}$, severe local surface plasticity can be observed which is shown in Fig. 4.23.

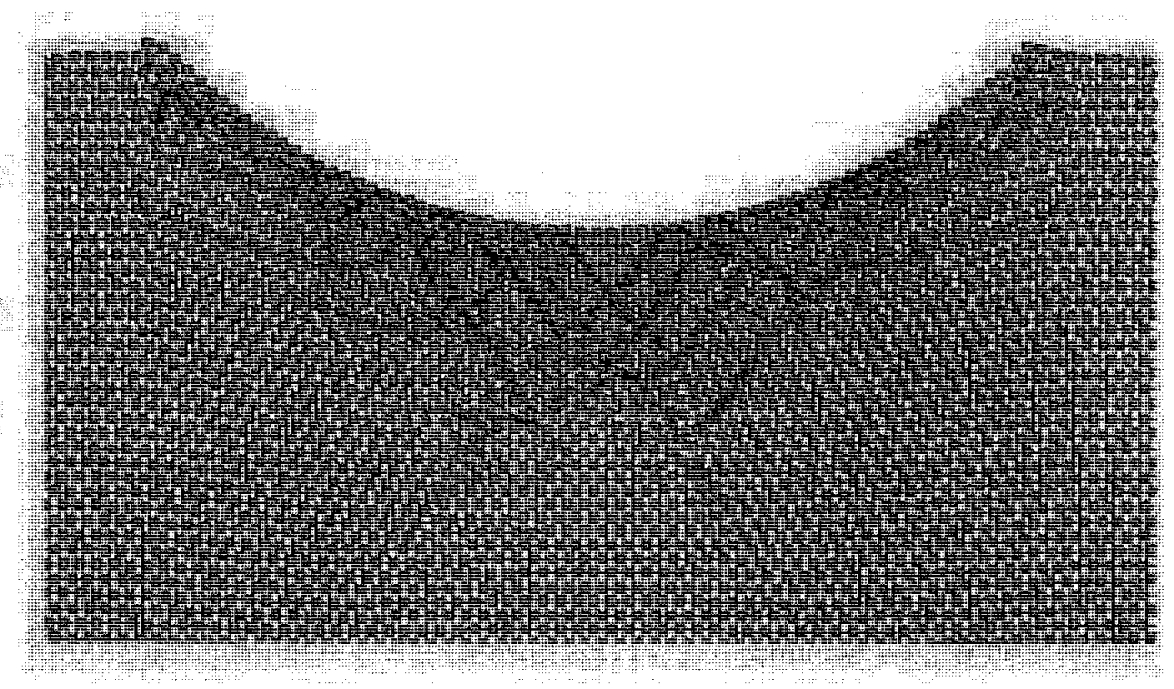

Figure 4.23: Local surface plasticity under high speed indentation $400 \mathrm{~m} / \mathrm{s}$. 


\section{Chapter 5}

\section{Material Removal Process at the Nanoscale}

There is an increase in demand for nano-parts in a wide range of potential applications (in the semiconductor, optical, electrical and mechanical industries) in which nano-surface finish is an important parameter. Consequently, the development of nano-scale surface material removal techniques such as AFM and ultra-precision machining or cutting have become a focal point of recent academic and industrial research [95].

Extensive experimental work has been carried out in metal surface plowing, cutting and finishing at the macroscale to develop, describe and optimize conditions and processes. This procedure is costly and time consuming. This was the motivation for looking at other alternatives to expand our understanding of these process while minimizing the experimental effort required. Material removal from the surface of nanometallic components is a more complicated manufacturing process in comparison to similar processes at the macro/micro level, and the fundamentals of mechanical surface manipulation at the nanoscale are not yet completely defined. Numerical simulations of surface material removal at the nanoscale provide researchers with a deeper understanding of chip formation mechanisms, scratching forces, size-effects, and machined-surface integrity. 


\subsection{State of Art of Modeling of Material Removal at the Nanoscale}

$F E$ and $M D$ are two key modeling methods that have been used to study the fundamentals of surface manipulation processes during the last decade. The $F E$ method is capable of predicting cutting or plowing forces, temperature, stresses and strains with a reasonable accuracy at the macrolevel, but as discussed before, $F E$ is not accurate enough on the micro or nanoscale. In this method, the workpiece material is considered a continuous structure neglecting the micro-constituents such as crystal structure, grain sizes and interatomic distances. To extend this work to nanoscales, atomistic level simulations are required to provide new data of various manufacturing process. Atomistics simulations allow the study of the influence of any one parameter by changing it and keeping the other characteristics unchanged. They can be applied to study conditions which cannot be investigated experimentally. Therefore, it is easy to find out the effectiveness of each parameter to obtain deeper understanding of the material removal process mechanisms at the nanoscale.

Recently, $M D$ has been applied to the study of the material removing process, especially nanoscale scratching and cutting. Inamura et al. [96] performed cutting simulations on single-crystal copper to find the effect of crystalline orientations and grain boundaries on cutting performance. Shimada et al. [97] tried to find the minimum chip thickness attainable from nanoscale cutting on copper with a diamond tool. Komananduri et al. [98] investigated nanoscratching on single crystal aluminum at extremely fine scratch depths (from $0.8 \mathrm{~nm}$ to almost zero). In their simulations, It is found that both the scratch and indentation hardness values decrease with increasing depth of penetration. Komanduri et al. [99] also studied the effect of the tool geometry in nanoscale cutting through $M D$ simulations with various tool edge radii. Belak [80] conducted $M D$ simulation of nanoscale plowing of a clean metal surface by a hard diamond tool in both two and three dimensions. Plastic deformation was found in the surface material and it was shown that dislocations 
propagate more readily in $2 D$ simulations than in the $3 D$ simulations. Shimizu et al. [100] studied plowing with $M D$ simulations in two dimensions. The simulation performed the contact of two surfaces without the formation of wear particles. They observed stick-slip phenomena during sliding, and also showed that the plowing speed was higher in materials with a higher elastic coefficient. Zhang et al. [101] investigated a $2 D$ diamond-copper system with the aid of $M D$ simulations. Their study revealed that there generally exists four regimes of deformation, i.e., the non-wear region, adhering regime, plowing regime, and cutting regime, based on the indent depth. The atomic-scale dislocation motion in each regime was also discussed. They pointed out that the slip lines generated by dislocation motion were very different from those predicted by slip-line of plasticity.

$M D$ simulations have difficulty analyzing large structures due to limitations in terms of time and length scales. For this reason, much of the work reported in the literature on $2 D$ or $3 D M D$ simulations of nanomachining are conducted on only several atomic layers and with high cutting tool speeds to reduce the computational effort [80]. Such limitations may cause some unwanted computational artifacts due to small model boundaries. To bring MD simulation closer to realistic conditions, the size of the simulation must be increased dramatically beyond that which has been presently reported. Not only is a large width necessary to study the cutting surface evolution, but the model must also be large enough in the other geometrical dimensions to capture short and long range effects of the interactions between the highly mobile dislocations generated ahead of the material removing tool. As the tool advances, more dislocations form at the tool-chip interface and penetrate deep into the work material. This requires that the size of the workpiece be increased considerably to avoid boundary effects, which come from artificial reflection of dislocations from the model's rigid boundaries. Dislocations which have been bounced from the simulation boundaries glide back toward the machined surfaces. This spurious phenomenon changes the real picture of dissociation and annihilation of mobile dislocations beneath the machined surface and thus changes the final profile of the surface behind 
the material removal tool.

Several methods have been developed to improve the $M D$ computational method such as Area-Restricted Molecular Dynamics $(A R M D)$ [102] and Length-Restricted Molecular Dynamics $(L R M D)$ [103]. However, no research has been done in using a multiscale model to simulate the material removal processes at the nanoscale [104].

To utilize the advantages and avoid the disadvantages of existing methods at various scales, we applied the $C A D D$ method to study the material removal process at the nanoscale. The computational efficiency of the method lets us study the plasticity of models with sizes greater than several hundred nanometers, subjected either to conventional nanoscratching and nanomachining $(1 \mathrm{~m} / \mathrm{s}$ to $25 \mathrm{~m} / \mathrm{s})$ [104] or super high-speed material removal process $(500 \mathrm{~m} / \mathrm{s}$ to $1000 \mathrm{~m} / \mathrm{s})$ [105]. In this study, we preserve full atomistic detail in the material removing region where atomistic-scale phenomena are expected to play an important role. We also investigate the long-range propagation of dislocations in the continuum region as discrete dislocations and the full interactions between the atomistic region (with all of its non-linear deformation and complexity) and the continuum defects.

\subsection{Multiscale Modeling of the Material Removal Process at the Nanoscale}

\subsubsection{Multiscale Simulations of Nanoscratching}

The model of nanoscratching along with the boundary conditions and the mesh used in the computations are shown in Fig. 5.1. A rigid tool with a perfect rounded tip is driven over the free surface of a block of aluminum atoms arranged in a $2 D$ triangular lattice as discussed in section 4.3.1.

The region close to the scratched surface is simulated with atomic resolution, while the remaining regions are simulated in a continuum sense, by finite elements. Using the multiscale model shown in Fig. 5.1 avoids the need for atomistic simulations containing 300,000 or more atoms; the simulation is performed with only 24000 atoms and 2500 


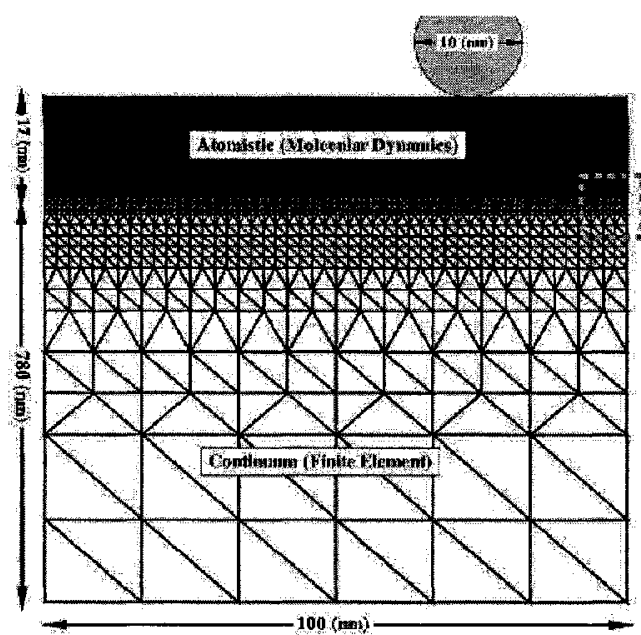

(a)

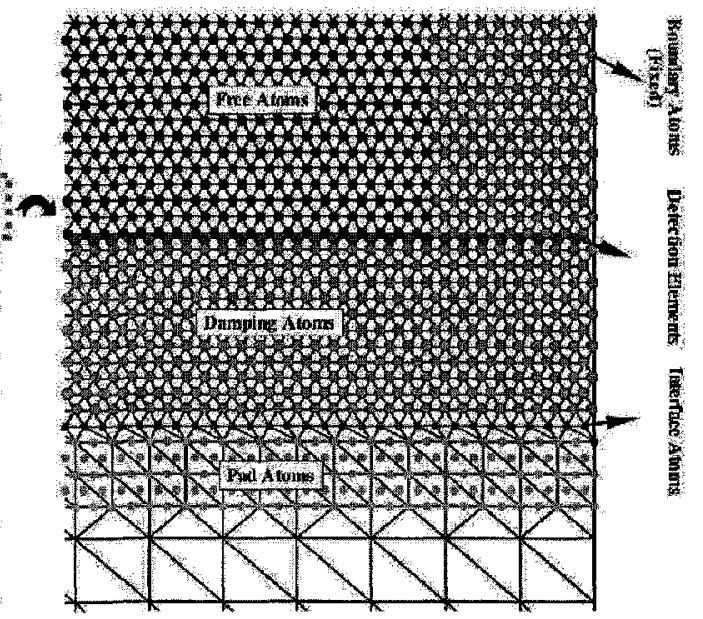

(b):

Figure 5.1: Multiscale model geometry and a close-up of the atomistic/continuum interface which shows continuum elements, free atoms, pad atoms, damping atoms and interface atoms. The circles that lie inside the continuum region are used as a "pad" of atoms to couple the atoms to the continuum region. The black elements inside the atomistic region are detection elements.

nodes. The tool is not modeled explicitly; rather, the circular tool has been defined as a repulsive potential of the form of Eq. (4.15).

Simulations are conducted to investigate the effect of using two initial scratching depths $(3.5$ and $4.6 \mathrm{~nm}$ ) with a tool tip radius of $5 \mathrm{~nm}$ on a triangular aluminum single crystal surface at room temperature. The crystal is oriented as shown in Fig. 5.1(b). The indenter distance from the right edge of the specimen in Fig. 5.1 is considered large enough so that edge boundary effects do not influence the results. The scratch length is chosen as $35 \mathrm{~nm}$. In order to simulate the scratching process, firstly, the tool is given a downward displacement which has a specified speed of $100 \mathrm{~m} / \mathrm{s}$ (indentation stage). Secondly, the downward displacement is taken at a slower rate at $2 \mathrm{~m} / \mathrm{s}$, and simultaneously a tangential displacement of $100 \mathrm{~m} / \mathrm{s}$ is also applied to the tool. Thermal relaxation is performed on the model before the tool is advanced towards the work material. The temperature in the damping band is held at $300 \mathrm{~K}$ using the Nose-Hoover thermostat.

Fig. 5.2(a) and (b) show views of scratching process and surface evolution for four dif- 

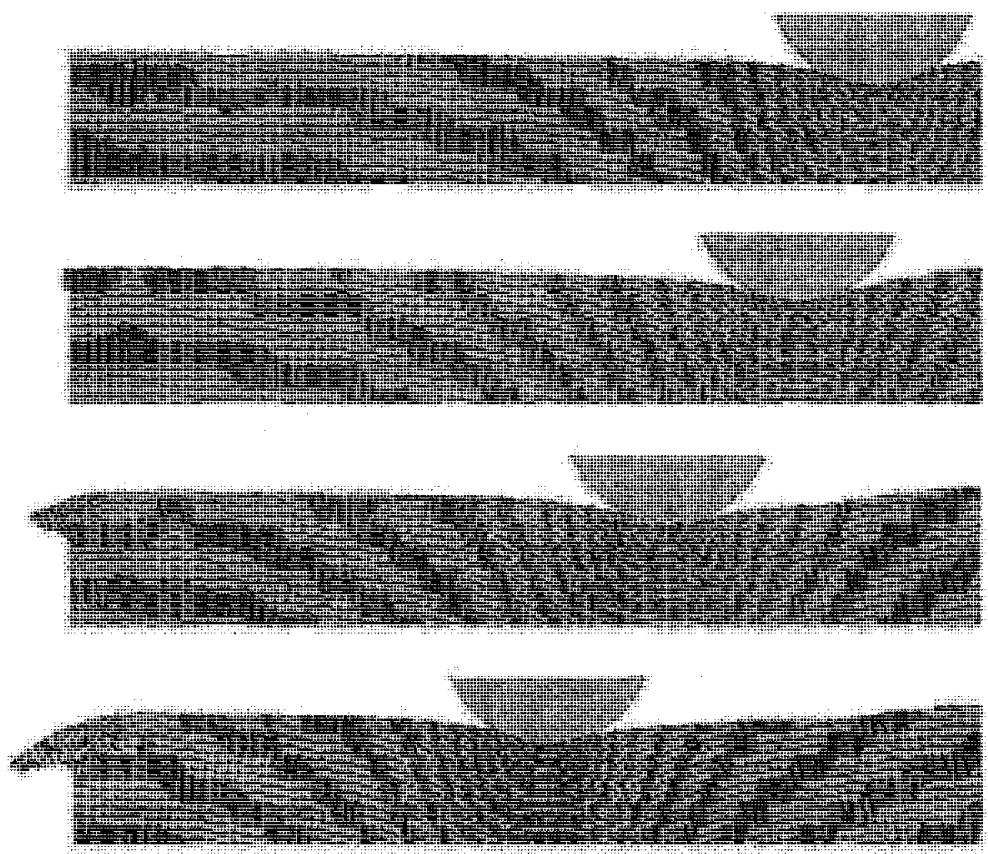

(a)
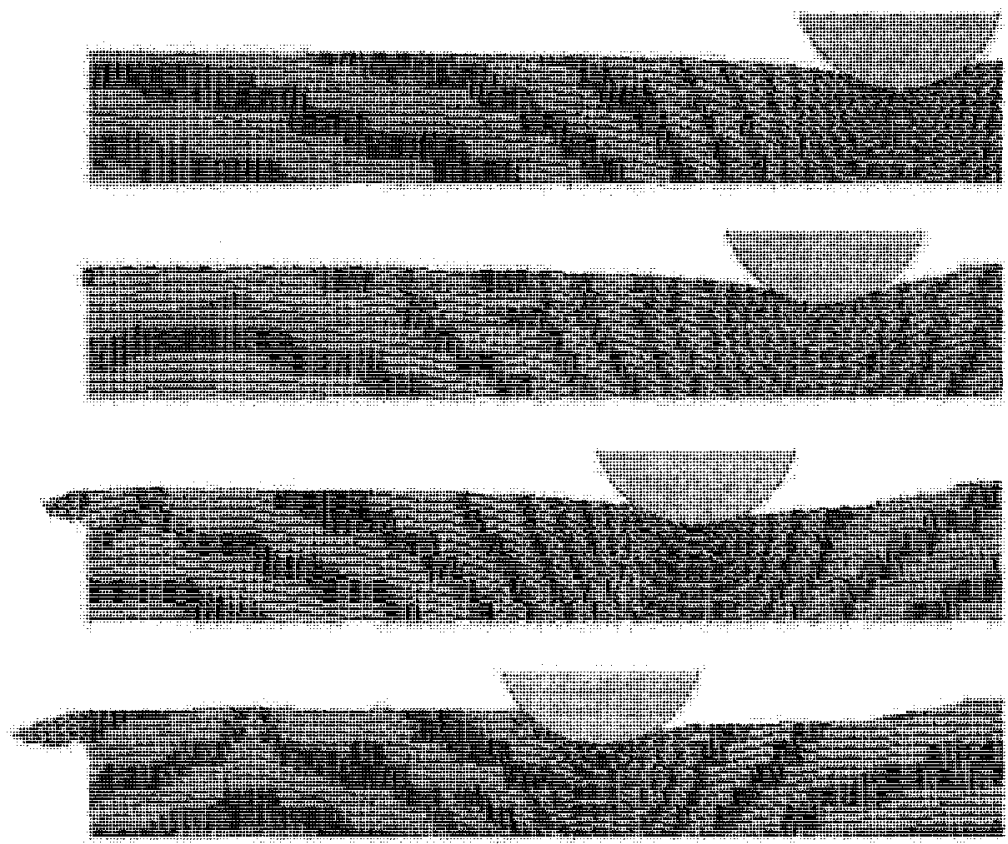

(b)

Figure 5.2: Snapshots of plastic deformation and surface finish for two different scratching depth (a) $3.5 \mathrm{~nm}$ (b) $4.1 \mathrm{~nm}$. The tool speed is $100 \mathrm{~m} / \mathrm{s}$. The scratching distance is $100 \mathrm{~nm}$ 
ferent time steps at two different initial scratching depths, 3.5 and $4.1 \mathrm{~nm}$, respectively. A simple comparison between the two figures shows that a higher degree of plastic deformation occurs beneath and ahead of the tool with a larger scratching depth. Close-ups of the scratching simulations of Fig. 5.2 are depicted in Fig. 5.3 and Fig. 5.4. Fig. 5.3(a) shows the dislocation arrangements due to slip of atoms under the tool during the indentation process. Note that the elements within the atomistic region in Fig. 5.3 are not used by the simulation in computing forces or energy; they are only used for the visualization of deformation. The meshes are defined in the perfect lattice reference configuration; hence the deformed finite elements highlight atomic planes on which slip has taken place. When a deformation such as dislocation motion takes place, the heavily deformed elements clearly show the effect on the structure. As was mentioned before, a single triangular aluminum crystal is studied here.

Dislocations which are generated at about $60^{\circ}$ to the horizontal direction are observed to penetrate deep into the work material, requiring the size of the workpiece to be large to avoid boundary effects. The final traveling depth of those dislocations is several times larger than the atomistic region thickness during the scratching process. The details of subsurface homogeneous nucleation of dislocations as dipoles and their propagations inside the atomistic region can be found in Ref [44]. Fig. 5.4(b) and (c) show the plastic deformation of the specimen due to dislocation propagation. In Fig. 5.4(b) and (c), the ploughing process plays a key role to generate slip planes under the tool. The dislocations at $0^{\circ}$ which travel deep and parallel to the ploughing direction toward the left side of the workpiece can be observed in Fig. 5.4(b) and (c). The phenomenon is intensified with further advance of the tool, as several layers of atoms are slipped beneath the tool to form the burr or side flow in the left end of the specimen as shown in Fig. 5.3(d). It has to be noted that there is no specified boundary condition on the left end edge of the model and the atoms are free to move.

Fig. 5.3(c) shows how the number of nucleated dislocations under the tool is increased 


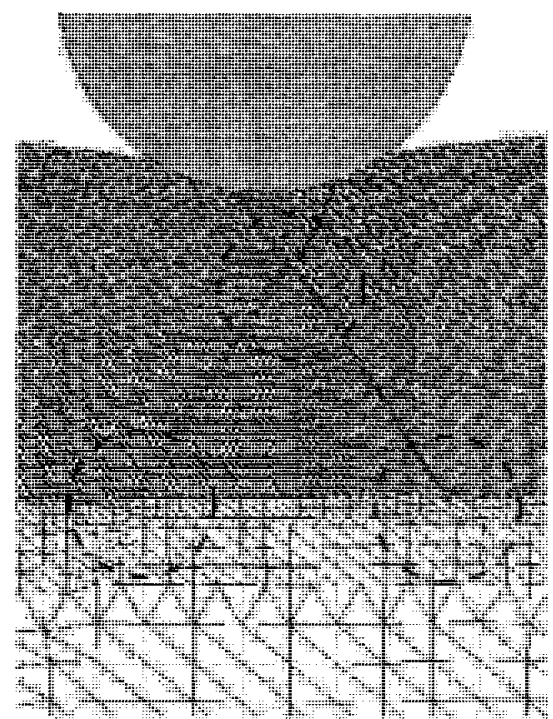

(a)

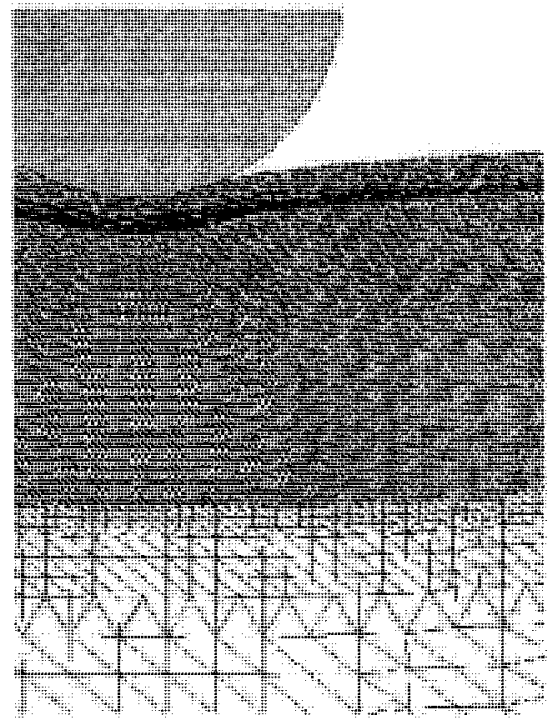

(c)

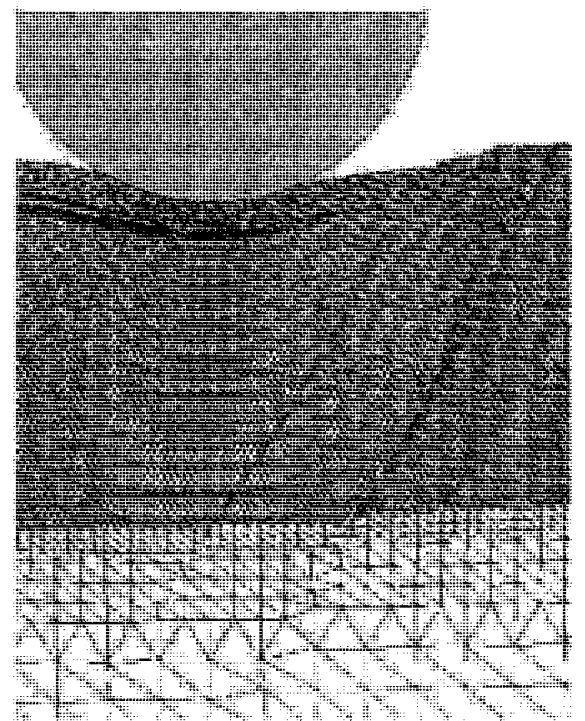

(b)

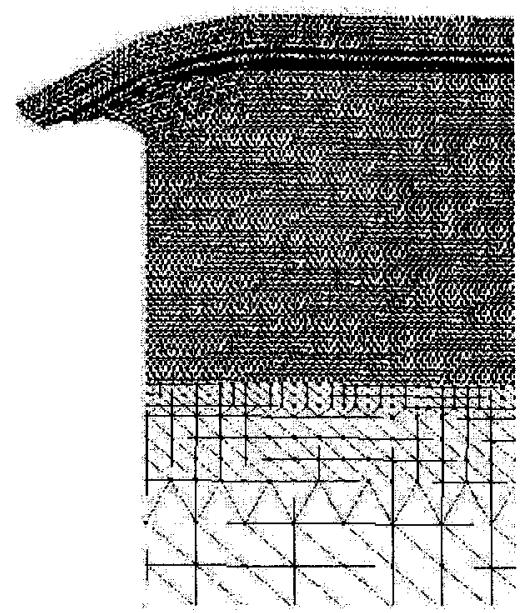

(d)

Figure 5.3: Close-up of generation and dissociation of dislocations. Scratching depth is $3.5 \mathrm{~nm}$. 


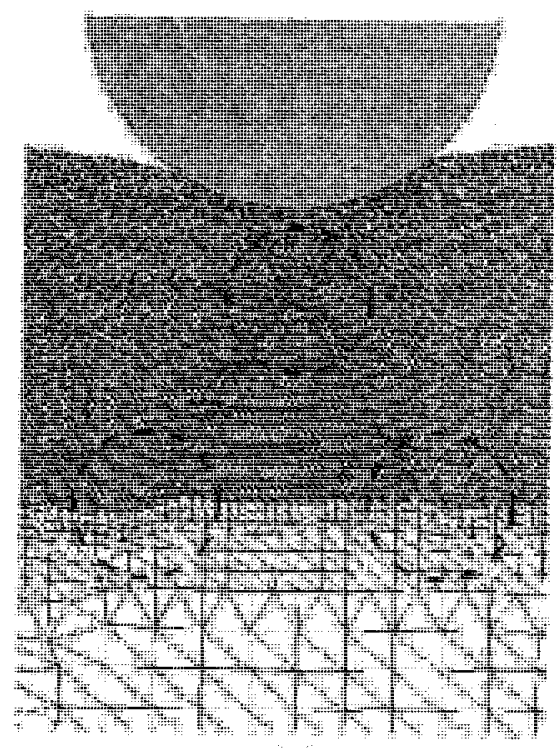

(a)

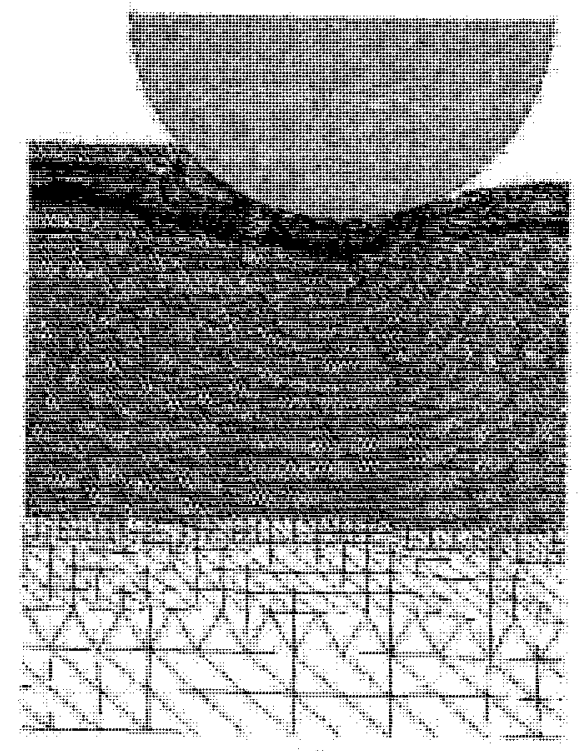

(c)

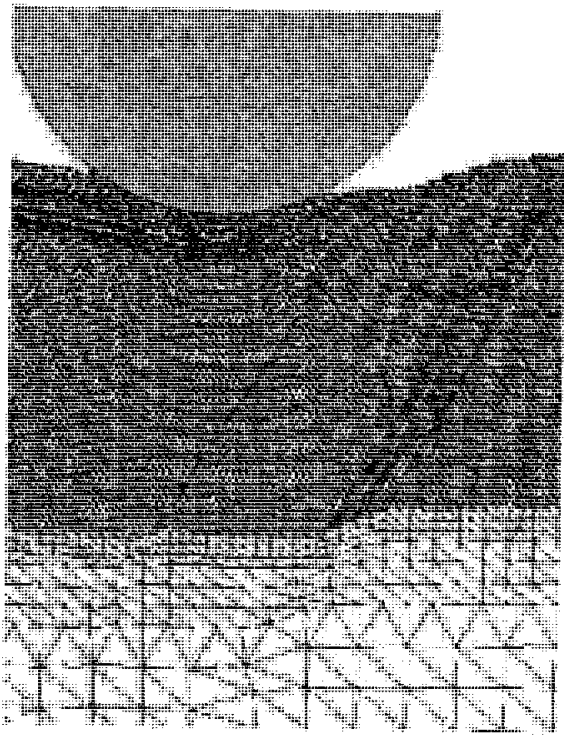

(b)

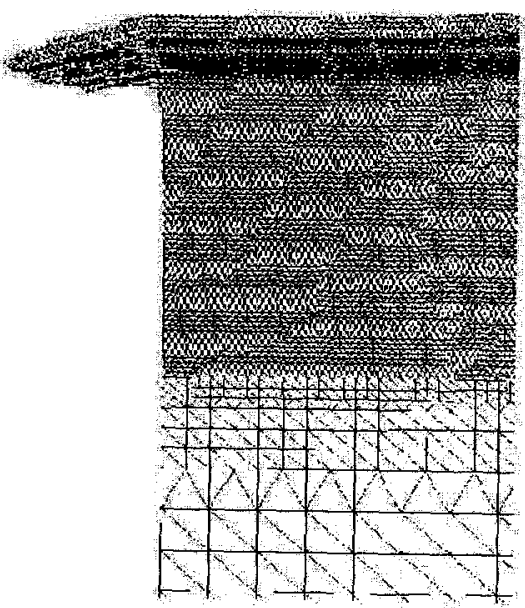

(d) Figure 5.4: Close-up of generation and dissociation of dislocations. Scratching depth is
$4.1 \mathrm{~nm}$. 
by advancing the scratching tool. In this stage, the dislocations are confined in a small band (about $2 \mathrm{~nm}$ ) near the surface. Then, for smaller scratching depth $(3.5 \mathrm{~nm})$, the major plastic deformation occurs in a small band close to the surface of the specimen. As it can be observed in Fig. 5.3(b) and (c), there is no dislocation migration to the continuum region in this case during tool advancement.

Fig. 5.4(a) shows that the number of homogenous nucleated dislocations is increased by increasing the indentation depth form $3.5 \mathrm{~nm}$ to $4.1 \mathrm{~nm}$. The multiscale model captures successfully all the moving dislocations toward the atomistic/continuum boundary and transfers them to the continuum discrete dislocations. The finite elements deformed because of the presence of discrete dislocations are indicated inside the dashed circles. Fig. 5.4(b) and (c) show the snapshots of the scratching process in two different time steps. A comparison of the Fig. 5.3(c) and Fig. 5.4(c) shows a higher degree of plastic deformation ahead of the tool with the deeper scratching depth. The continuous generation of slip in the direction of the depth under the scratching tool can also be observed in Fig. 5.4(c). The slip planes not only are located under the tool but also ahead of the tool.

Another issue that should be noted is that the EAM aluminum single crystal acts like a ductile material and does not exhibit phase transitions during plastic deformation. Dislocations appear during the compression state and many of them disappear during elastic recovery as the tool moves forward. Based on simulations of the scratching process, the dislocations generated in aluminum are found to be highly elastic in nature. The layers of atoms move upwards and result in elastic recovery, so a roughened surface is generated. In other words, the roughness surface generation is only a natural result of dislocation propagation inside the workpiece material during two major compression and relaxation stages.

Figs. 5.5 and 5.6 demonstrates the variation of normal and tangential forces versus time for two different scratching depths after using a smoothing technique to remove thermal noise. As can be seen in Fig. 5.5, the graph can be divided into two regions. In 


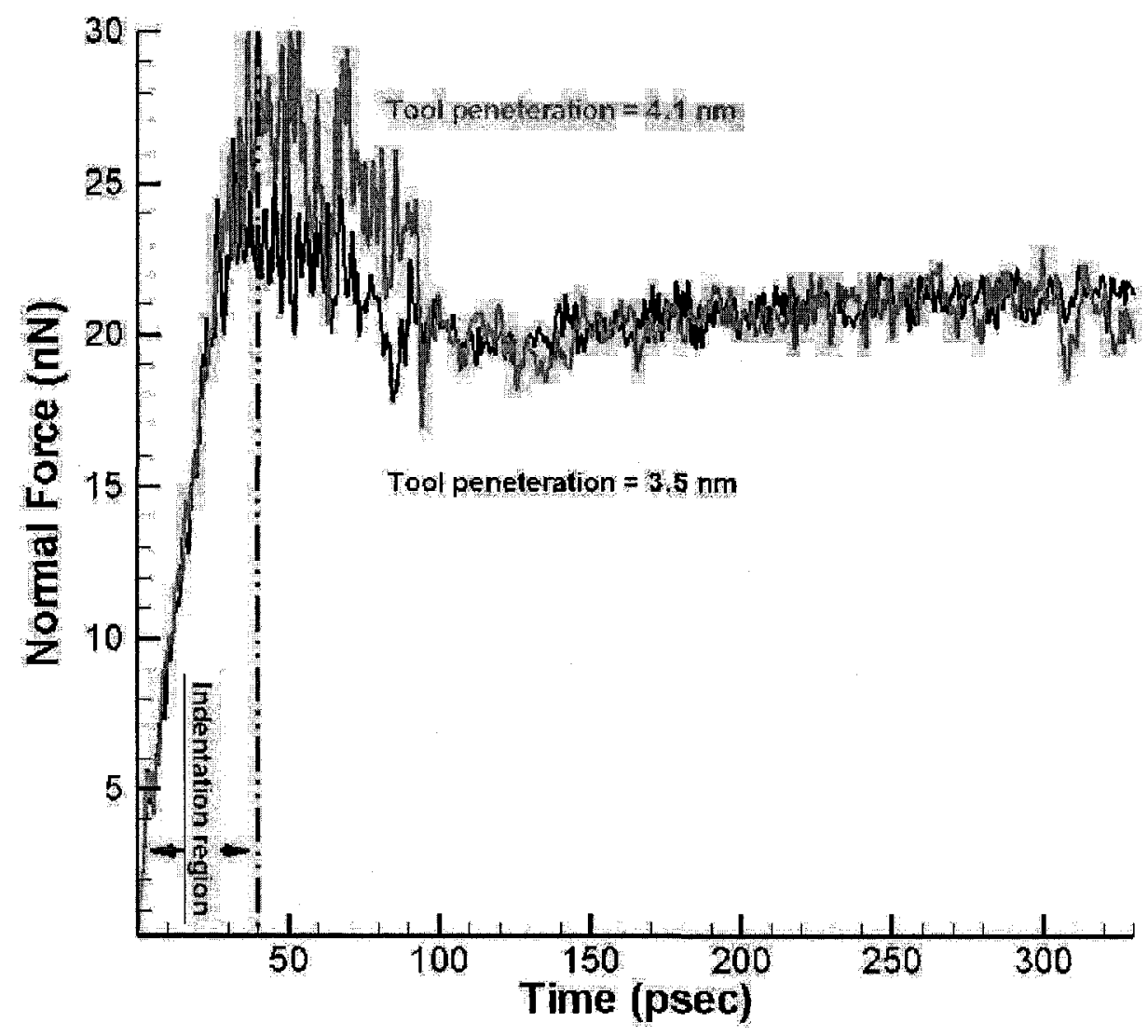

Figure 5.5: The variation of normal force of single aluminum crystal for two different scratching depths. 


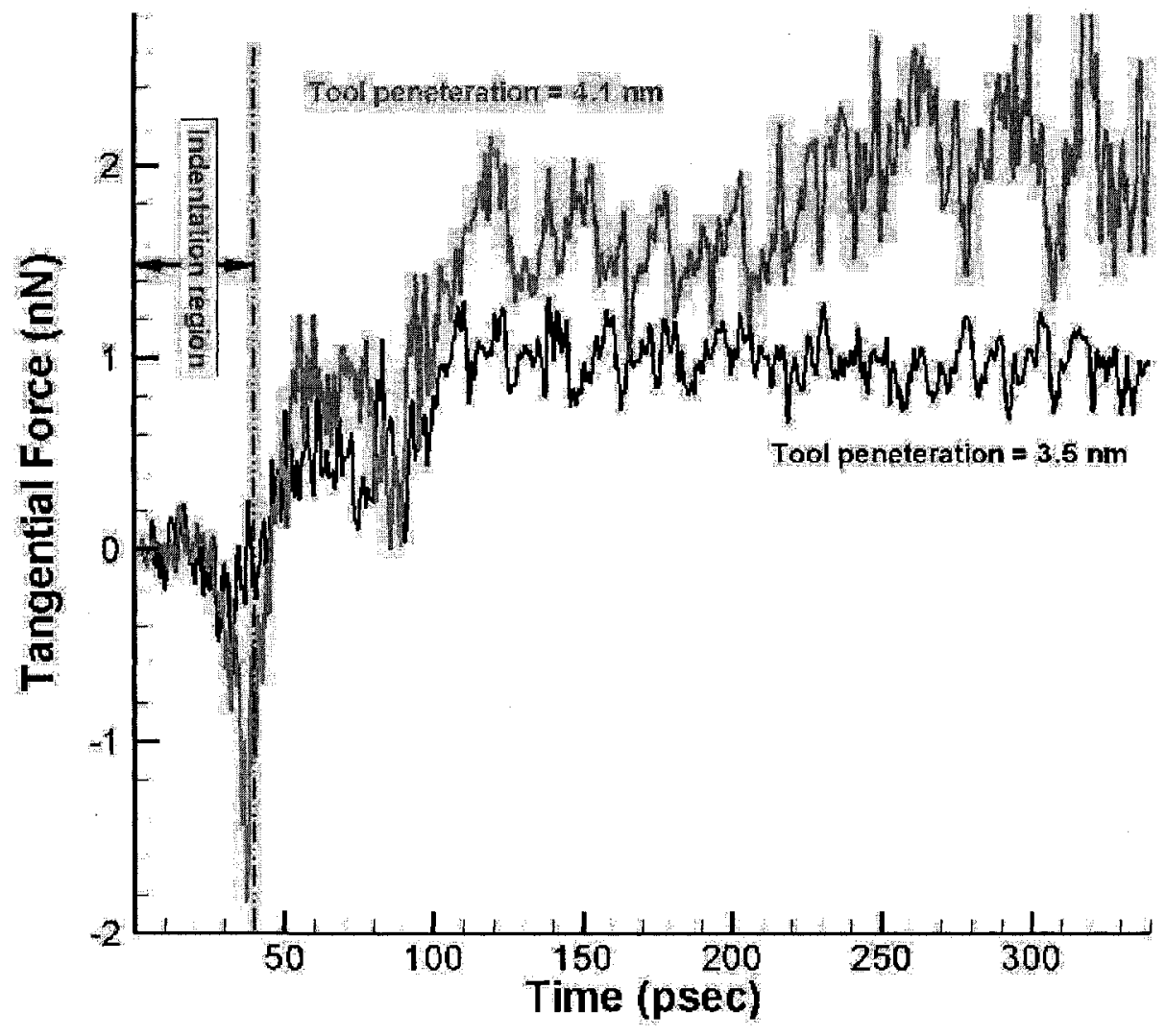

Figure 5.6: The variation of tangential force of single aluminum crystal for two different scratching depths. 
the first region, indentation occurs prior to tool advance. In this region, the normal force for both penetration depths increases by the downward advancing of the tool. The wellknown normal force dropping during homogenous nucleation of dislocation beneath the indenter can be observed in the indention region of the curve [44]. In the end of the indention process, the normal force is higher for the deeper indention test. In the second part of the graph, the normal force drops gradually for both scratching depths for a short period. This drop is caused by elastic recovery of the workpiece after ending the indentation stage. The normal force increases gradually by the same trend of increasing the vertical tool penetration motion. It should be noted that for the variation of normal force versus time is almost the same for both scratching depths.

As it is expected, the tangential force during the indentation process remains almost zero, but a considerable drop of tangential force happens in the end of the indention process and beginning of ploughing process. The specimen's elastic recovery in the end of the indention stage can be an explanation for this drop. The tangential force increases rapidly in a short duration by starting the ploughing stage for both scratching depths. The average of tangential force remains almost constant for smaller scratching depth after the first sharp rise, but the tangential force increases by increasing the ploughing stage for larger scratching depth. This difference can be explained by comparing Fig. 5.3(c) and Fig. 5.4(c). There is no pileup of surface atoms in the front of the tool for the smaller ploughing depth, but the accumulated atoms in the front of the tool for larger ploughing depth show additional resistance against the tool motion in the tangential direction as shown in Fig. 5.4(c). The tangential force also experiences a noticeable fluctuation because of numerous complicated processes of generation, dissociation and annihilation of dislocations under and ahead of the tool for the larger scratching depth. 


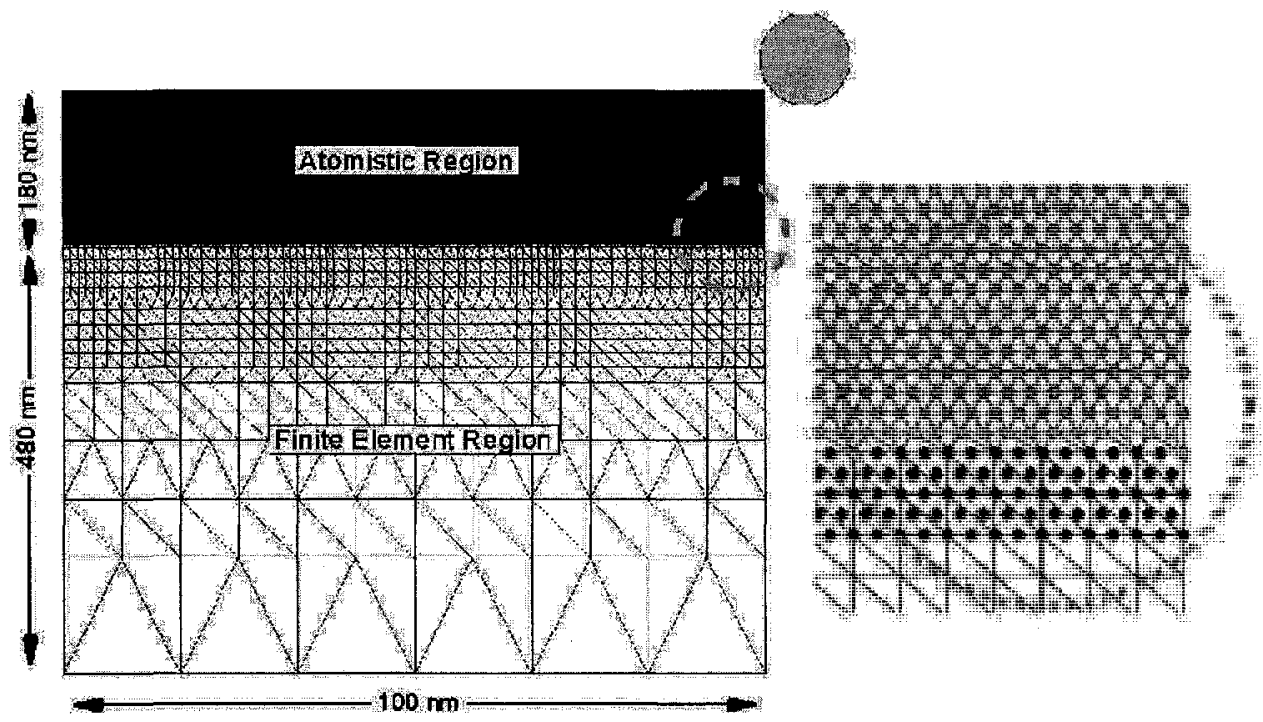

Figure 5.7: Multiscale model geometry and a close-up of the atomistic/continuum interface which shows continuum elements, free atoms, pad atoms, damping atoms and interface atoms.

\subsection{Multiscale Modeling of Nanocutting}

In most classical $M D$ simulations [97], there is an extremely high interaction between the tool and work atoms which makes it difficult for the tool atoms to maintain a crystal structure during the material removal process. The tool atoms can be easily melted or evaporated during heavy material removal. In this study, we have ignored tool wear, owing to the uncertainty of the friction at the tool-workpiece interface. Instead, a rigid cutting or grinding tool is considered.

In this section, we discuss the removal process of material from an aluminum single crystal substrate surface, as shown in Fig. 5.7, at room temperature (300 K). Thermal relaxation is performed on the model before the tool is advanced towards the work material. The temperature of the damping band atoms is set to $300 \mathrm{~K}$. 


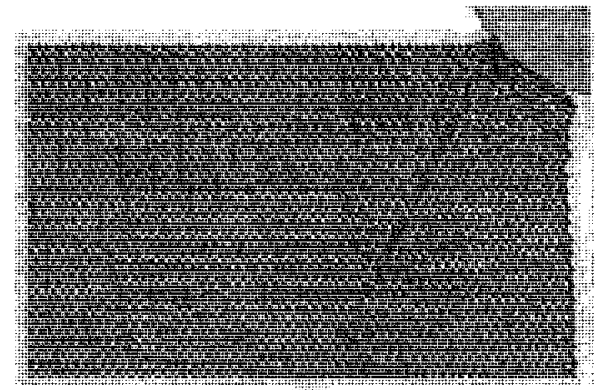

t+4:

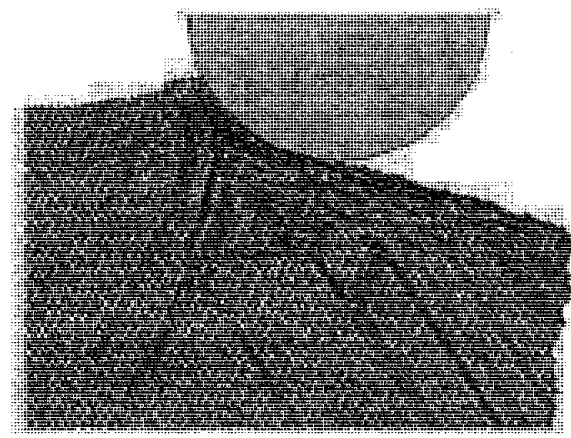

(c)

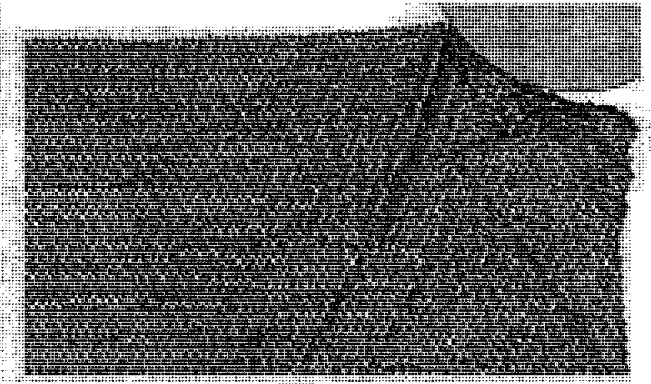

14

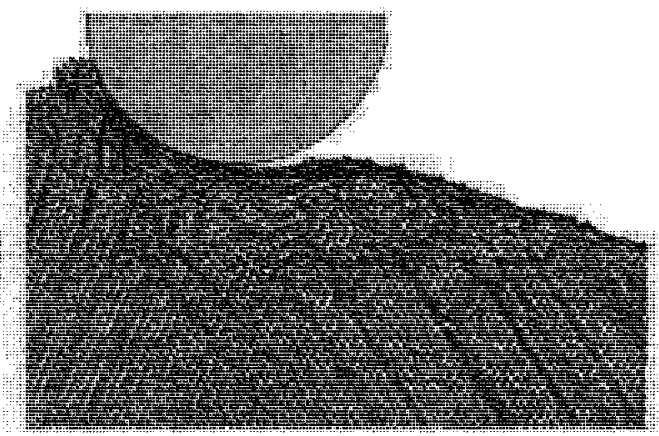

(d)

Figure 5.8: Simulation plots showing various beginning stages of scratching of an aluminum single crystal. Scratching depth, tool speed and tool radius are $1.7 \mathrm{~nm}, 100 \mathrm{~m} / \mathrm{s}$ and $5 \mathrm{~nm}$, respectively.

Fig. 5.8 shows the initial state of removal of material at four different time steps. The depth of scratching is $1.7 \mathrm{~nm}$ and the tool moves forward at $100 \mathrm{~m} / \mathrm{s}$. Fig. 5.8(a) shows the dislocation arrangements due to slip of atoms under and ahead of the tool. Note that the apparently large slope of the deformed surface is an effect induced by the free surface on the right edge of the model, and quickly levels off as we move deeper into the workpiece (as we will see later in Fig. 5.10).

The dislocations which are generated at about $60^{\circ}$ to the ploughing direction are observed to penetrate deep into the work material, requiring a very large workpiece to avoid boundary effects. The coexistence of ploughing and cutting processes can also be seen in Fig. 5.8(b-d). In Fig. 5.8(b)-(c), the ploughing process plays the key role where atoms are piled up and extruded behind and in front of the tool, a feature of the ploughing state. The 
phenomenon is intensified with further advance of the tool, as atoms in front of the tool are piled-up as a layer forming the chip as shown in Fig. 5.8(d). This figure presents the state in which cutting plays the key role as the volume of the chip becomes larger and the surface is degenerated in a continuing material removal process. The layer of atoms that appears on the front of the tool causes tangential and normal forces on the tool during the material removal process which will be discussed in detail later.

In addition to the complicated processes of generation, dissociation and annihilation of dislocations, two distinct periods, a compression period beneath the tool and an elastic recovery period after the tool has passed by, can be observed during the material removal process as already observed in [99]. As a result of tool advancement in each time step, high compressive and shear stresses cause the breaking of atomic bonds. The extrusion action of the compressive stress in front of and underneath the tool leads to deformation, dislocations, and the rearrangement of the crystal lattice. The dislocations which are generated in this stage glide away from the nucleation spots in the compression process, and then they glide back toward the relaxed structure as the tool passes. After a long period of relaxation for the surface material removal, the final machined surface will be generated.

Fig. 5.9 illustrates the ability of the developed multiscale method to capture all above features of the process. The depth of scratching is $2.0 \mathrm{~nm}$ and the tool advances $100 \mathrm{~m} / \mathrm{s}$. Fig. 5.9 shows the dislocation distribution for two different time periods which can capture the compression and relaxed states, respectively. As shown in Fig. 5.9(a), at least five of the dislocations penetrate very deep into the workpiece in compression state, three of which (\#1-3) are detected by the detection band and successfully passed to the continuum supporting region as discrete dislocations. Fig. 5.9(b) is a snapshot of the distribution of dislocation after the tool passes further along top of the region shown in Fig. 5.9(a). As it can be seen, with the tool advancement, four out of five numbered dislocations in Fig. 5.9(a) glide back from continuum to the atomistic region and disappear into the free 


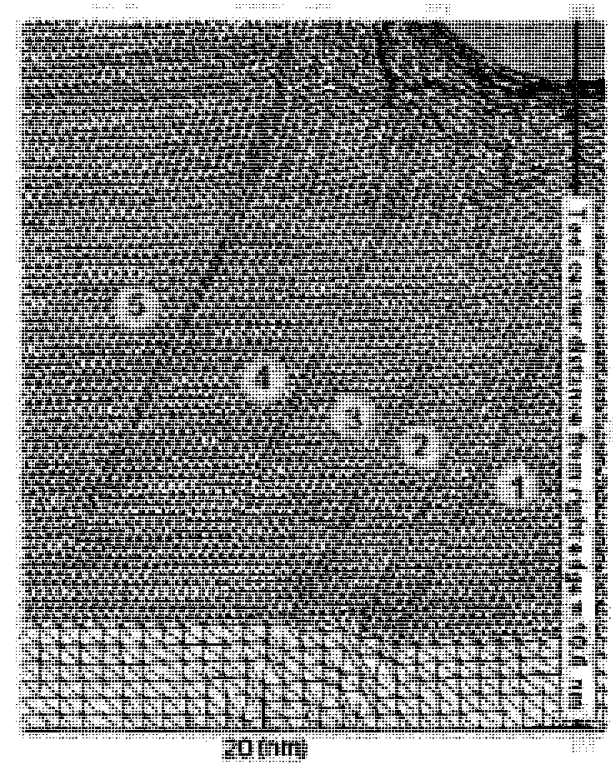

(a)

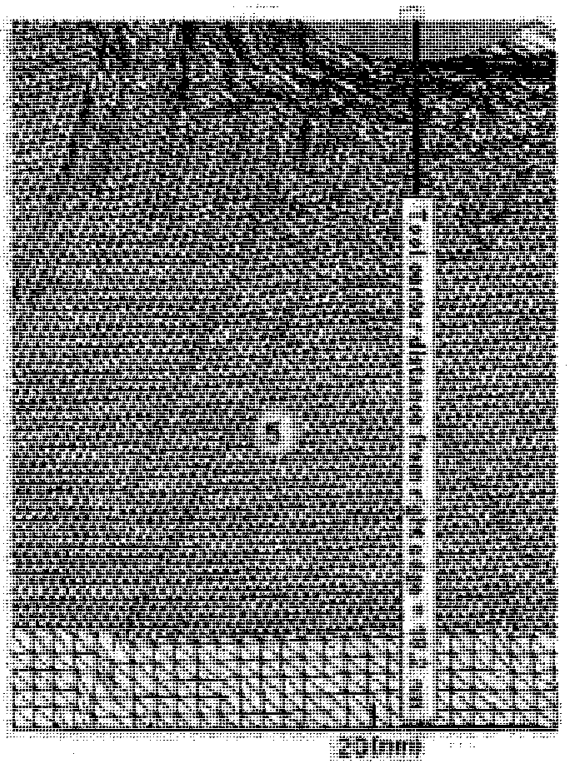

(b)

Figure 5.9: Distribution of dislocations in two different states: (a) compression state, (b) relaxed state. Numbers highlight slip traces in the way of dislocations, the cores of which are at the end of each slip trace. In (a), dislocations 1-3 are below the field of the view, deep in the continuum region. In (b) the relaxed area is located in the right side of the red line.

surface. The fifth dislocation is forced to move deeper into the workpiece because of the tool advancement.

The above simulations of the material removal process show that the dislocations generated in the $E A M$ aluminum substrate are highly elastic. Dislocations appear during the compression state and many of them disappear during elastic recovery as the tool moves forward. As a result of the successive generation and disappearance of dislocations, the chip seems to be removed step by step. The layers of atoms move upwards and result in elastic recovery, so the rough machined surface is generated. The chip removal and the machined surface generation are directly connected to dislocation motion inside the substrate materials during two major compression and relaxation stages.

Simulations are also conducted to investigate the effect of using various ploughing 


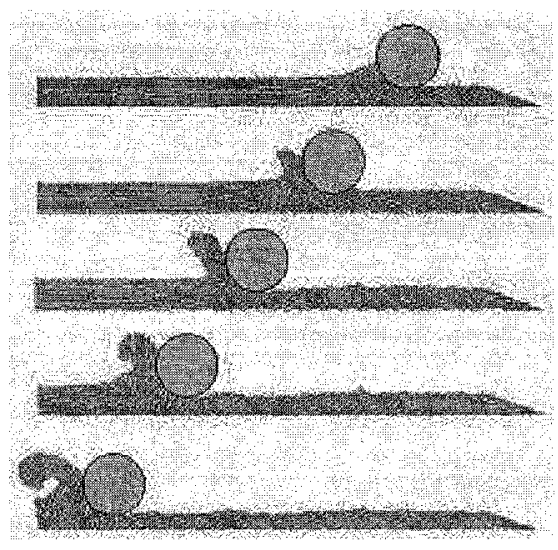

(a)

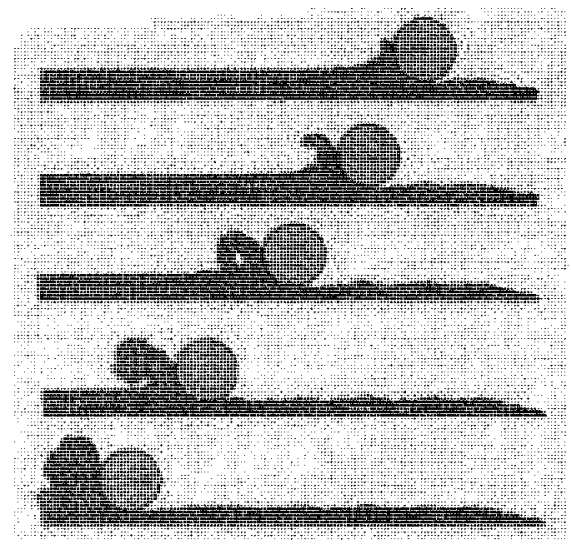

(b)

Figure 5.10: Snapshots of chip deformation and surface finish for two different scratching depths: (a) $1.7 \mathrm{~nm}$, (b) $2.0 \mathrm{~nm}$. Tool speed is $100 \mathrm{~m} / \mathrm{s}$. The scratching distance is $100 \mathrm{~nm}$.

depths $(1.5,1.7$ and $2 \mathrm{~nm}$ ) with a tool tip radius of $5 \mathrm{~nm}$. The tool advances $100 \mathrm{~m} / \mathrm{s}$. Fig. 5.10(a \& b) show views of ploughed surface and chip deformation for five different time steps at two different ploughing depths, 1.7 and $2.0 \mathrm{~nm}$, A comparison of the different figures shows a higher degree of plastic deformation ahead of the tool with a larger ploughing depth. The number of nucleated dislocations under and in front of the tool is also increased by increasing the ploughing depth. Fig. 5.10 shows a long ploughing distance of about $100 \mathrm{~nm}$. In these snapshots, the chip grows in a stepwise manner to reach a critical height. After this stage, it starts to roll over to the free surface of the workpiece ahead of the tool. As a result of the tool advancement, chip atoms and the surface atoms combine together and reconstitute to form a new chip geometric profile. This process can not actually be seen in a real material removal process in air because the surface atoms on the chip are oxidized quickly. A model which can capture this realistic state of the removal process is a subject of current research.

Fig. 5.11 and Fig. 5.12 demonstrate the variation of normal and tangential forces for two different scratching depths (after thermal smoothing technique to remove thermal 


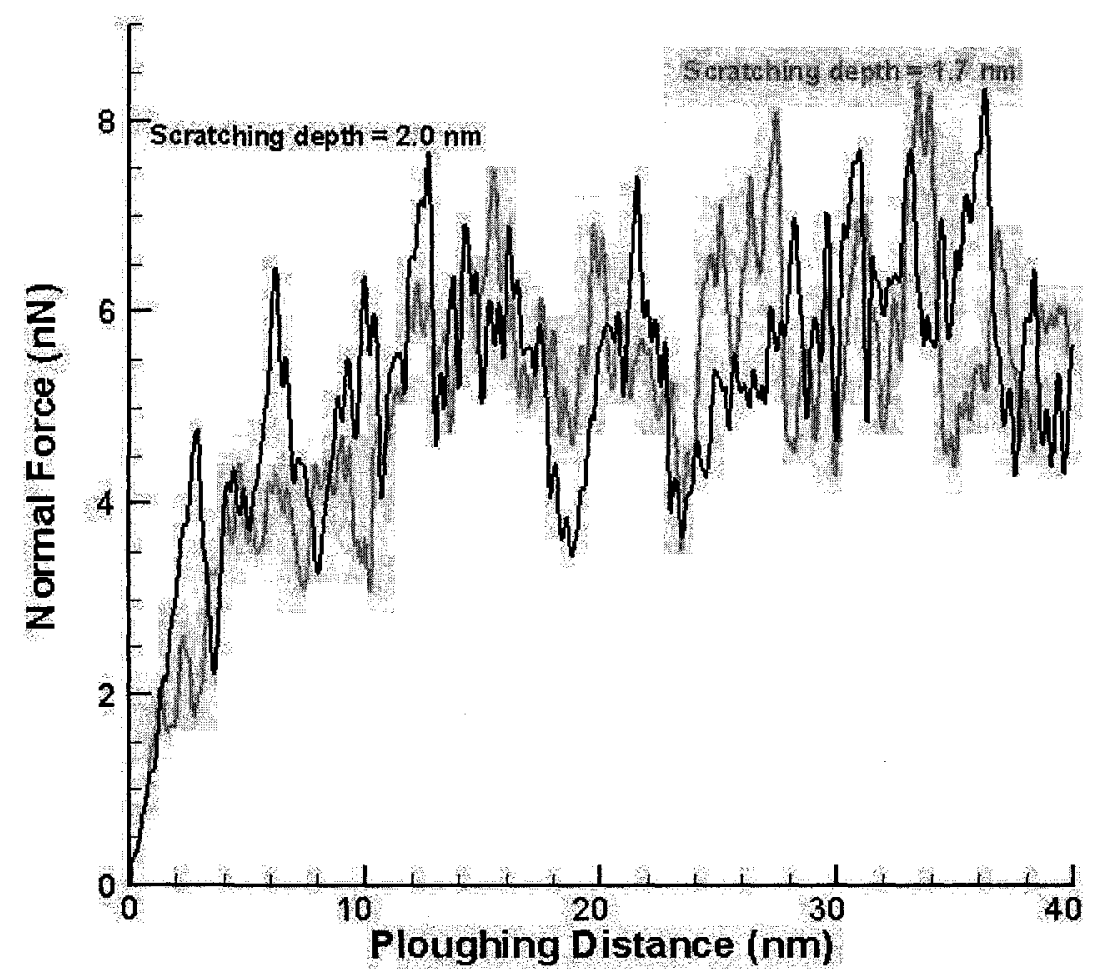

Figure 5.11: The variation of normal force of single aluminum crystal for two different scratching depths, 1.7 and $2.0 \mathrm{~nm}$, respectively.

noise). As can be seen in Fig. 5.11 and Fig. 5.12, the simulation with a larger ploughing depth exhibited higher tangential and normal forces during the material removal process. The relative increases of the average tangential and normal forces are $17 \%$ and $12 \%$, respectively, for a $0.3 \mathrm{~nm}$ increase of scratching depth. By careful smoothing to reduce only noise and lessen sharp fluctuations in the data, the major fluctuation of the forces can still be observed in Fig. 5.11 and Fig. 5.12. Those drops and rises of the forces values occur because of dislocation initiation events during the material removal process.

Critical points on the tangential force curve are labeled by roman numerals in Fig. 5.12. From the start of the ploughing process to point $I$, the response is almost elastic. Some dislocations are initiated at about $60^{\circ}$ to the ploughing direction as shown in Fig. 


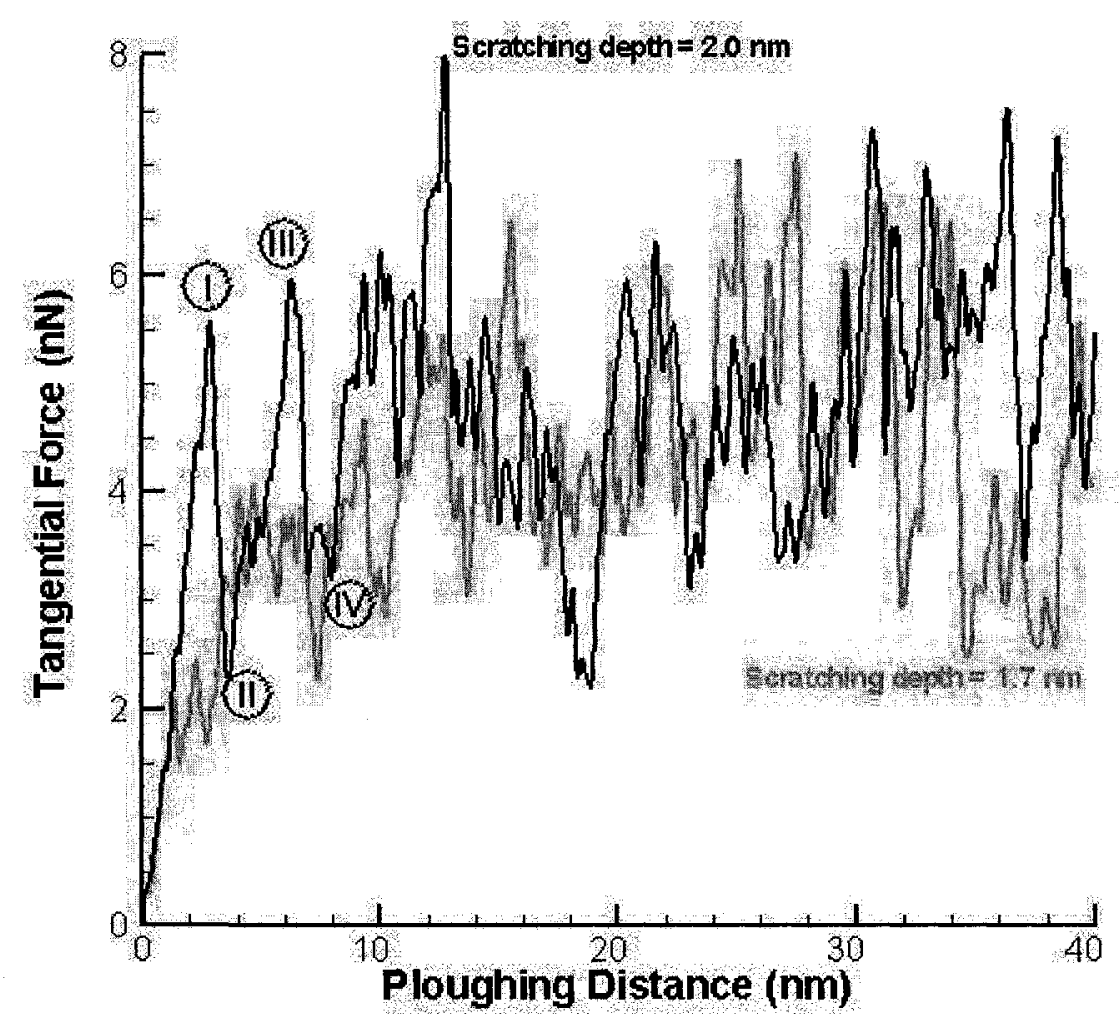

Figure 5.12: The variation of tangential force of single aluminum crystal for two different scratching depths, 1.7 and $2.0 \mathrm{~nm}$, respectively. Points $I-I V$ are critical features described in the text.

5.13(a), but all of them will disappear if the tool is removed and the workpiece experiences almost a complete elastic recovery. The first huge drop of tangential force happens from $I$ to $I I$ which corresponds to the nucleation of some dislocations at $60^{\circ}$ to the ploughing direction and one dislocation at $0^{\circ}$ which travels very deeply parallel to the ploughing direction toward the left side of the workpiece as shown in Fig. 5.13(b). The second huge drop of tangential force occurs from III to IV which corresponds to the nucleation more dislocations at $60^{\circ}$ to the ploughing direction as shown in Fig. 5.14(a) and one dislocation at $0^{\circ}$ which travels deep and parallel to the ploughing direction toward the left side of the 


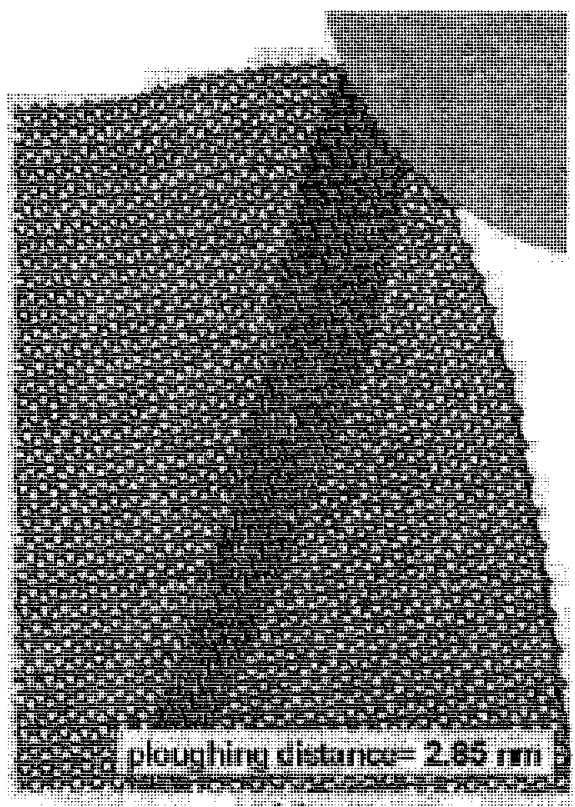

to:

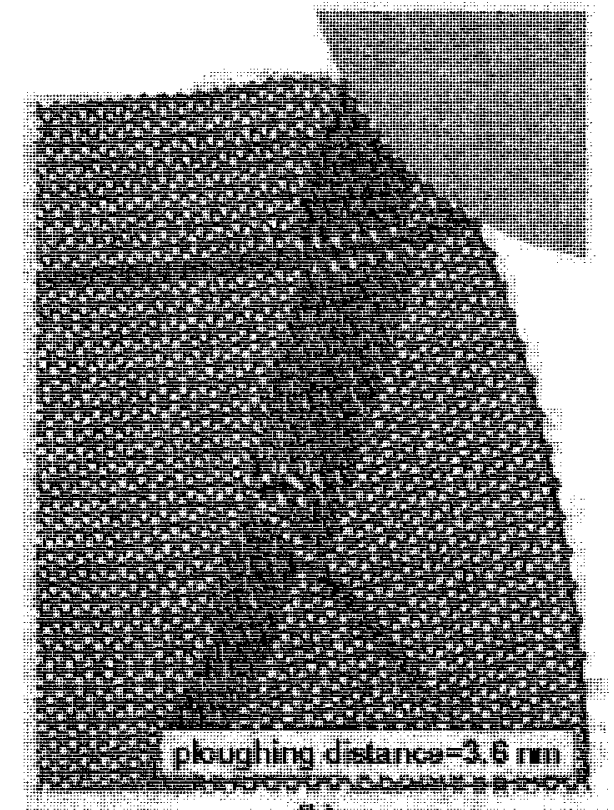

(b):

Figure 5.13: Snapshots of generation of dislocations between points I and II on the tangential force curve.

workpiece as shown in Fig. 5.14(b). Animated sequences of the dislocation nucleation and propagation from point $I$ to $I I$ and point $I I I$ to $I V$ show the nucleation of dislocation at $0^{\circ}$ parallel to the ploughing direction are mainly responsible for the first tangential force drop in Fig. 5.12.

To study the effect of tool speed on the material removal process, simulations are performed for a range of tool speed from $200 \mathrm{~m} / \mathrm{s}$ to $800 \mathrm{~m} / \mathrm{s}$. Fig. 5.15 shows snapshots of the evolution of the ploughed surface and chip formation processes under various high scratching speeds at five different time steps where (a)-(d) are the results of the simulation with tool speed $200,300,400,800 \mathrm{~m} / \mathrm{s}$, respectively. The depth of scratching is fixed at $2 \mathrm{~nm}$ for all the various tool speeds. In the case of Fig. 5.15(a), many atoms accumulate in front of the tool and make a chip. In this case, the dislocations can travel significantly 

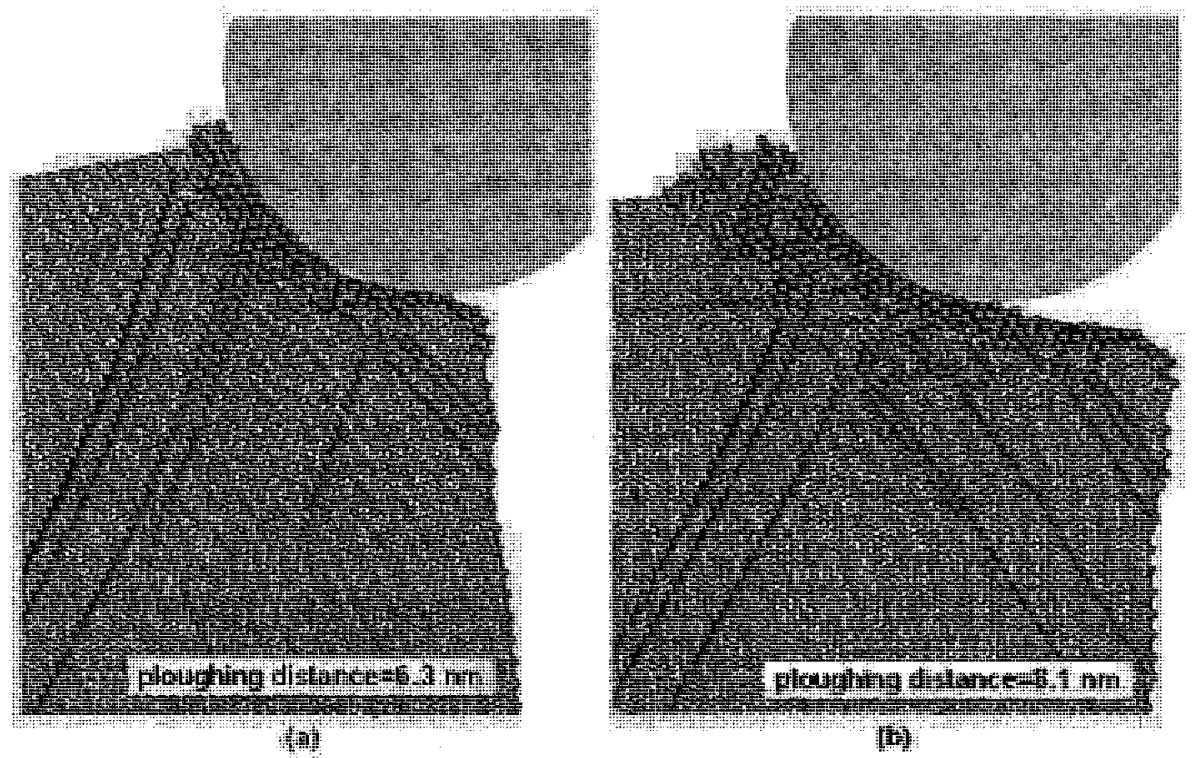

Figure 5.14: Snapshots of generation of dislocations between points III and IV on the tangential force curve.

far from the front of the tool as observed for the simulation at $100 \mathrm{~m} / \mathrm{s}$ as shown in Fig. 5.10(b). These dislocations are considered to be a cause of the burr or side flow in the finished surface after process as shown in Fig. 5.16. In the condition of Fig. 5.15(a), the number of generated slip planes in the direction of the depth is smaller than the number of initiated dislocations at $100 \mathrm{~m} / \mathrm{s}$. In the case of Fig. 5.15(b) in which the ploughing speed is $300 \mathrm{~m} / \mathrm{s}$, the height of the chip becomes higher than that of Fig. 5.10(b) and Fig. 5.15(a). The slip planes are less than in the case in Fig. 5.15(a) which are generated to the depth direction of the workpiece. By increasing the velocity of the tool, dislocations cannot clearly nucleate. As a result, in the high velocity simulations, the deformation of the substrate under the tool is highly localized.

In the case of Fig. 5.15(c) in which the tool speed is in the range of the propagation speed of a plastic wave [106], the cutting chip formation observed is different from Fig. 5.15(a) and (b), and the accumulation of non-crystalline atoms in the front of the tool 


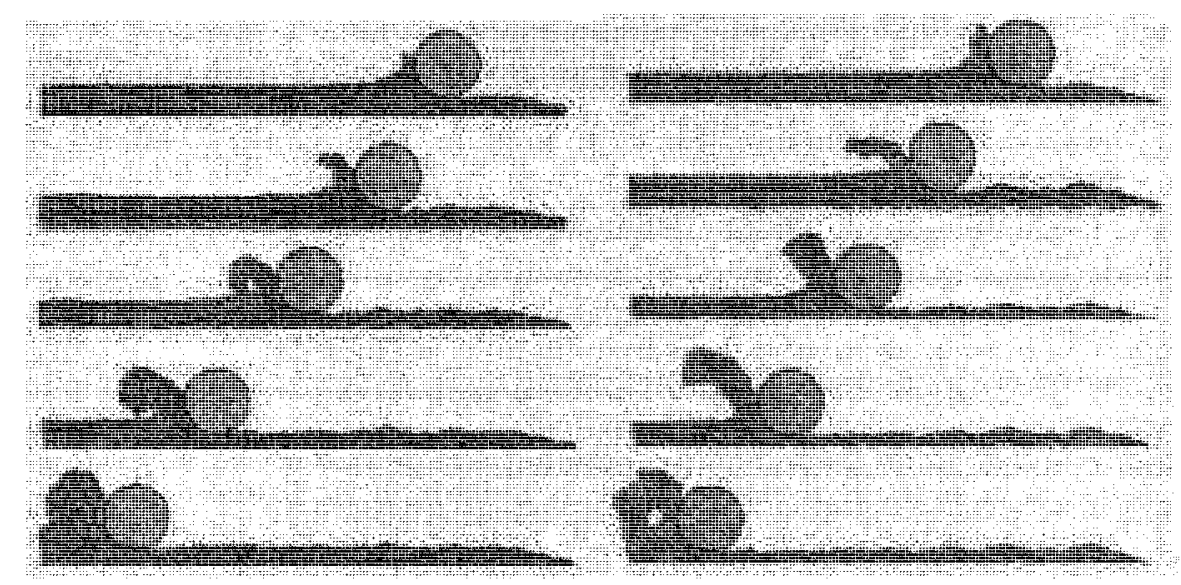

(6)

(b)

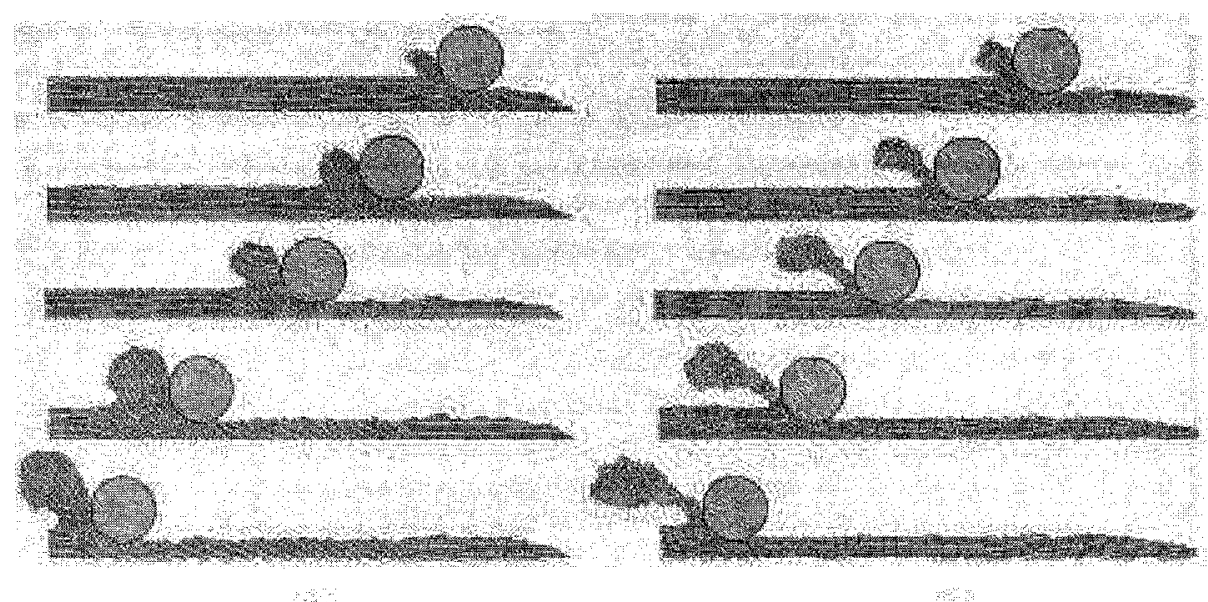

(c)

(d)

Figure 5.15: Snapshots of chip deformation and surface finish in scratching process for four different tool speed: (a) $200 \mathrm{~m} / \mathrm{s}$; (b) $300 \mathrm{~m} / \mathrm{s}$; (c) $400 \mathrm{~m} / \mathrm{s}$; (d) $800 \mathrm{~m} / \mathrm{s}$. The scratching distance is $100 \mathrm{~nm}$. The depth of scratching is $2.0 \mathrm{~nm}$.

becomes shorter but thicker than those in Fig. 5.15(a) and (b). The number of the active slip planes decreases further than the case in Fig. 5.15(a) and (b), as well. At these speeds, the motion of dislocations indicating plastic deformation of the substrate surface can no longer be clearly identified. Instead, local amorphization followed by plastic flow is a more accurate description of the surface plasticity. In the case of Fig. 5.15(d), in which the tool speed is certainly higher than the propagation speed of a plastic wave, formation 


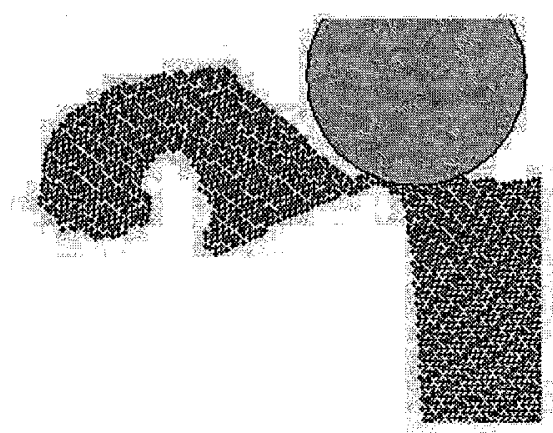

(a)

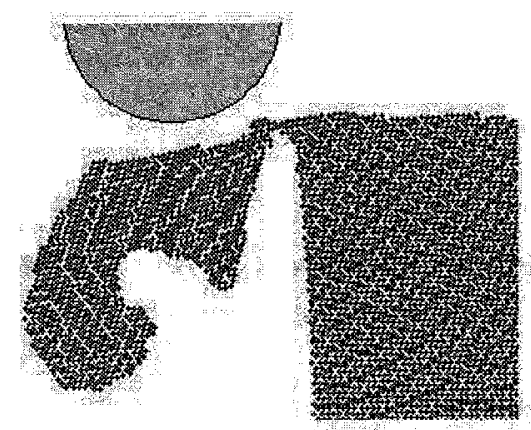

(c)

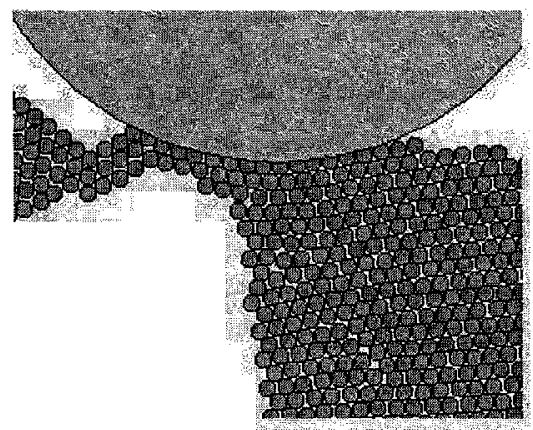

(b)

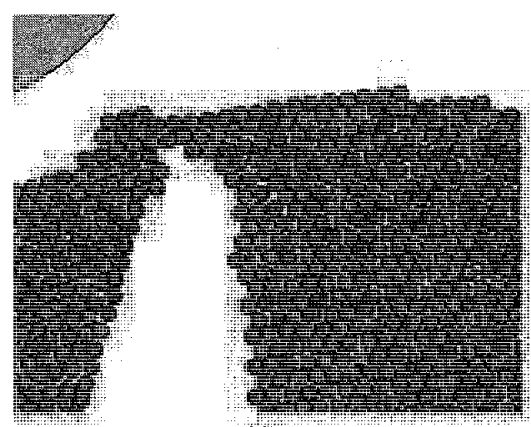

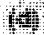

Figure 5.16: Exit-burr formation at final stage of material removal process. The scratching depth is $2.0 \mathrm{~nm}$ and tool moves $100 \mathrm{~m} / \mathrm{s}$. Close-up of exit-burr formation is shown in (b) and (d).

of a cutting chip is clearly different from Fig. 5.15(a-c).

Fig. 5.17 shows a close-up of the chip profile and surface finish for two tool speeds, $200 \mathrm{~m} / \mathrm{s}$ and $800 \mathrm{~m} / \mathrm{s}$. It can be seen that the chip structure changes from a crystalline form in Fig. 5.17(a) to a non-crystalline form in Fig. 5.17(b). At $800 \mathrm{~m} / \mathrm{s}$ tool speed, the atoms in the cutting chip have lost their crystallinity, the number of the active slip planes in the depth direction of the workpiece is very small and all of them are located very close to the surface. Plastic deformation in front of the tool is hardly observed. From these results, it can be understood that the material deformation mechanism greatly changes, when the tool speed exceeds the propagation speed of plastic wave of the workpiece material [106]. The plastic wave speed of a crystal depends on the plastic flow or plastic strain, and as 


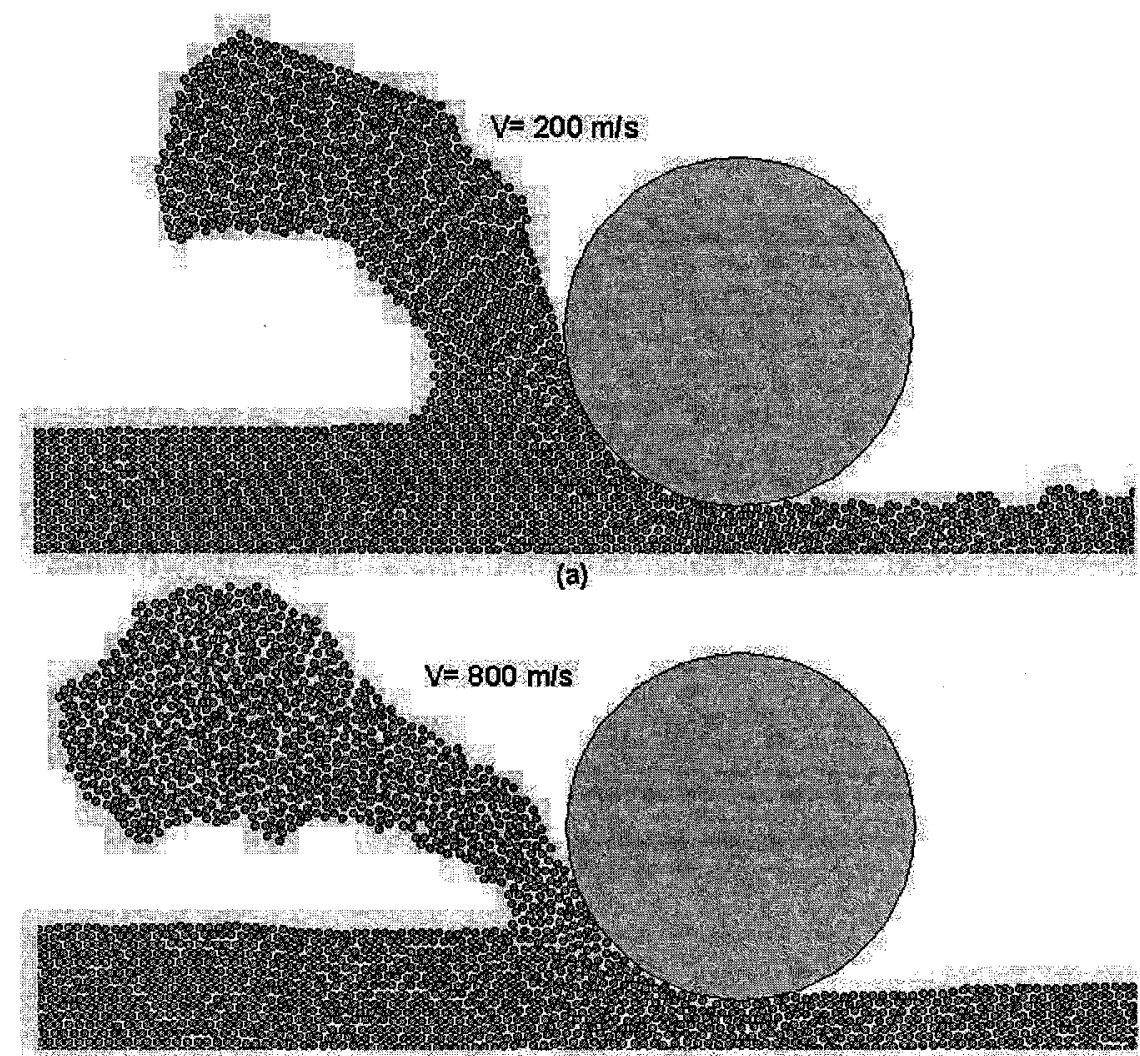

(b)

Figure 5.17: Close-up of chip profile and surface finish for two different tool speed: (a) $200 \mathrm{~m} / \mathrm{s}$; chip has a crystalline structure, (b) $800 \mathrm{~m} / \mathrm{s}$; chip shows an amorphous structure. The depth of scratching is $2.0 \mathrm{~nm}$.

such the exact plastic wave speed is not easy to calculate for the current case. However, one can find a good estimation of plastic wave speed based on knowing the elastic wave speed in the crystal. The plastic wave speed is slower than elastic wave and becomes at least $\frac{1}{10}$ of elastic wave. The calculated elastic wave in this particular workpiece is 4619 $\mathrm{m} / \mathrm{s}$, so that the upper value for the plastic wave speed is about $460 \mathrm{~m} / \mathrm{s}$.

It seems that for a very high speed material removal process, a thin atomistic model will work well due to the lack of dislocations which may travel deeply through workpiece. Hence, applying the developed multiscale model to simulate very high speed material 


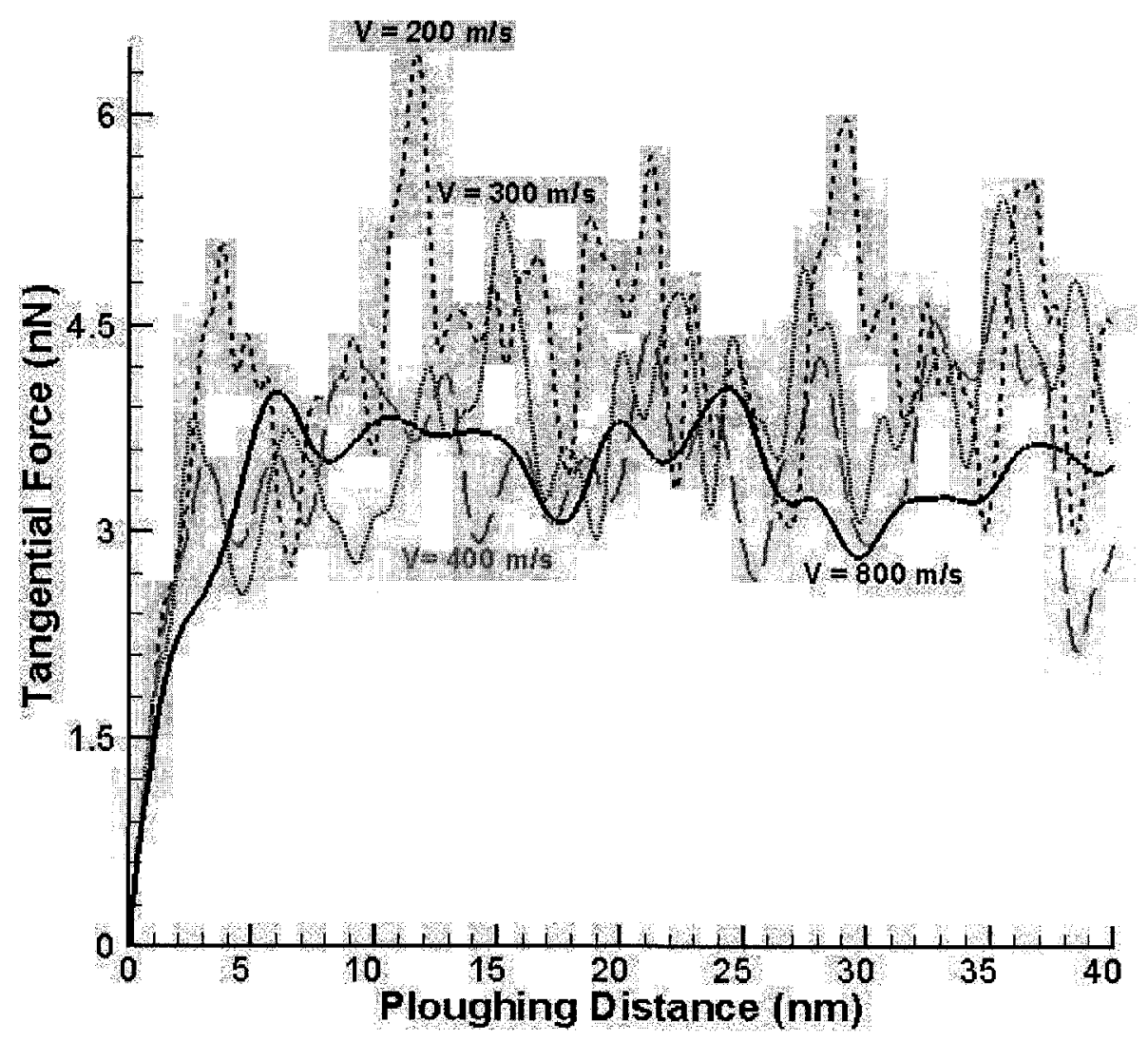

Figure 5.18: The variation of tangential force of single aluminum crystal for four different tool speeds. Scratching depth is $2.0 \mathrm{~nm}$.

removal processes is not necessary. The conducted simulations above $400 \mathrm{~m} / \mathrm{s}$ tool speed show that the multiscale model is no more efficient than classical $M D$ simulations to reveal the details of surface removal processes. The multiscale approach is important, however, for tool speeds below about $400 \mathrm{~m} / \mathrm{s}$.

Fig. 5.18 and Fig. 5.19 show the variation of the tangential and normal forces versus ploughing distance. The fluctuation of the forces is decreased by increasing the tool speed. This is in agreement with the relationship between force fluctuations and dislocation generation events during removal process. As was mentioned before, dislocation 


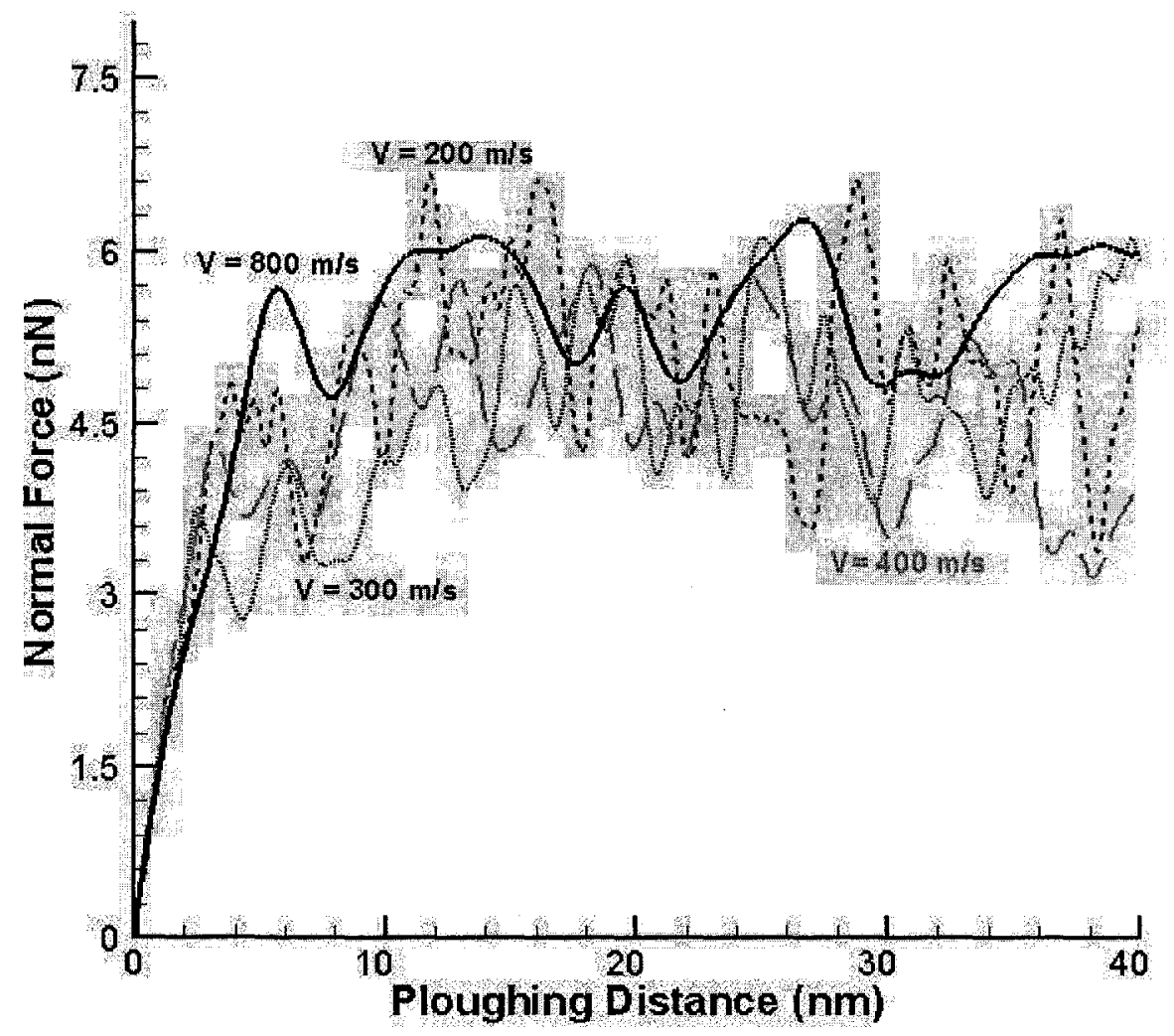

Figure 5.19: The variation of normal force of single aluminum crystal for four different tool speeds. Scratching depth is $2.0 \mathrm{~nm}$.

propagation becomes limited when the tool speed is higher than the propagation speed of plastic wave. The quality of the scratched surface is also significantly different in low and high speed simulations. Increasing the speed of the tool increases the smoothness of the machined surface. As discussed previously, the roughness is due to dislocations returning to the surface after the tool has passed.

The average tangential and normal forces are shown in Fig. 5.20 as a function of tool speed where both average forces are the mean values over the range of in ploughing distance. Initially, the average tangential and normal force is decreased by increasing the tool speed as shown in 5.20 to provide a guide to the eye. The least square method is used to 
determine the best curves to fit the series of data points in Fig. 5.20 . Up to $400 \mathrm{~m} / \mathrm{s}$ in scratching speed, the average normal force is decreased, and it takes a sudden increase at $800 \mathrm{~m} / \mathrm{s}$. The observed differences are related to the transition from crystal plasticity due to lattice dislocation motion to an amorphization process, as previously discussed. In static or quasi-static applications, the mechanism for stress-induced amorphization and the structural details and properties of the resulting amorphous solid are topics that are often currently discussed in the physics and material science literature [107]. One proposed mechanism that is strongly supported by experimental observations is that stress-induced amorphization results from a violation of the Born conditions for crystal stability [108]. These criterion state that the determinant of the coefficient matrix for the elastic moduli has to be non-singular. This leads to a set of inequalities for the elastic moduli that determine crystal stability. The instability of the crystal lattice leads to a collapse of the crystal and the concomitant production of the amorphous solid composed of a high concentration of defects and dislocations. It appears that such a loss of stability occurs in our simulations at high tool velocities. This observed phenomenon is already under an additional investigation. 


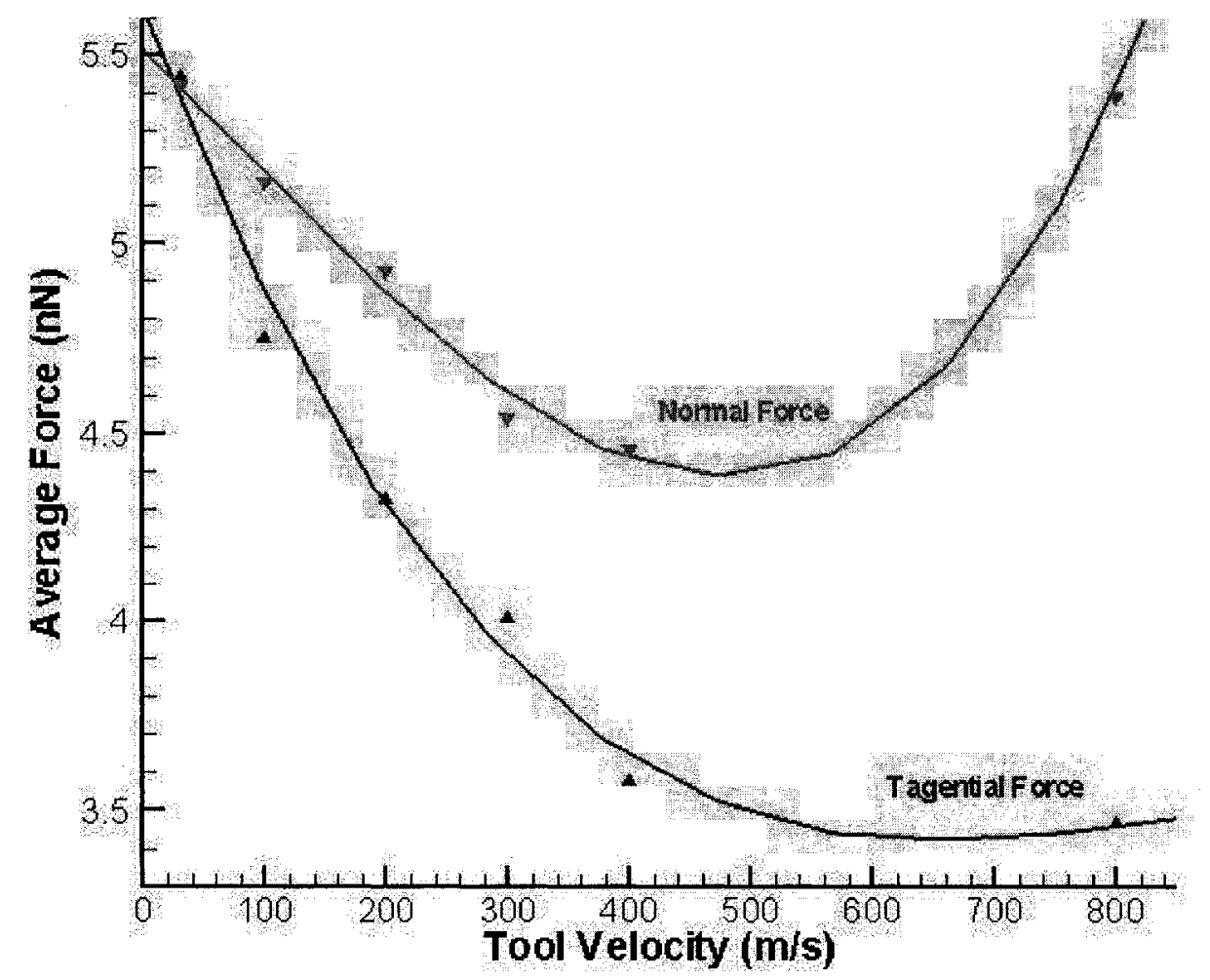

Figure 5.20: Variation of average tangential and normal forces versus tool speed. The scratching depth is $2.0 \mathrm{~nm}$ and data are collected for $100 \mathrm{~nm}$ plowing distance. 


\section{Chapter 6}

\section{Conclusions and Future Recommendations}

\subsection{Conclusions}

Materials modeling remains one of the most important challenges facing today's mechanics, materials science and physics communities. With the growing use of advanced materials in industry there is a pressing need for viable computational and theoretical approaches to assist in the design of such materials. Real materials are highly complex, involving structure on many different length scales. The observed macroscopic behavior is often a function of the collective microstructure of the material with no one length scale that can be singled out as dominant. This places many problems out of reach for both traditional continuum and atomistic methods. This thesis is devoted to the development of a method, called the dynamic coupled atomistic/discrete dislocation (CADD) method, which allows the modeling of materials involving complex multiscale phenomena.

In Chapter 2, we discussed how the passage of mechanical waves across any boundary between numerical methods can cause spurious reflections. Any interface between the atomistic and continuum regions will cause reflections, so some means must be used to minimize or dampen these reflections. We have presented a numerical damping technique in one dimension. Wave propagation examples in $1 D$ illustrated the effectiveness of the damping band method in eliminating potentially spurious high frequency atomistic waves 
at the $M D / F E$ interface. We have also shown that the method can dissipate the second type of the reflection originating from the discrete continuum region.

In chapter 3 , we have presented the details of the dynamic $C A D D$ method which is capable of supporting discrete dislocations in the continuum region. In addition, the method includes algorithms to automatically detect dislocations as they want to move across the atomistic/continuum interface, and correctly converts dislocations between the two domains. By implementing a damping band along the atomistic/continuum interface, there is no spurious heating due to phonon reflection at the interface. The CADD method simultaneously extends the realm of applicability of both fully atomistic simulations and discrete dislocation approaches. For atomistic methods, dynamic $C A D D$ allows for larger systems to be studied without the need to explicitly treat every atom in the problem. While other coupled methods can provide similar benefit, dynamic $C A D D$ is well-suited to problems where dislocations want to travel over long distances. This is often the case in ductile metals with high dislocation mobility and low Peierls barriers. Dynamic CADD extends the size of the simulation to macroscopic length scales, serving as a dislocation sink and isolating the atomistic region from simulation boundary artifacts. On the other hand, $C A D D$ extends the range of problems accessible to discrete dislocation methods by reducing the dependence on phenomenological rules for dislocation nucleation.

In Chapter 4, the dynamic $C A D D$ is tested and applied to the nanoindentation process. In addition to the usual advantage of coupled methods, i.e., the reduction of the computational overhead compared to atomistic simulation, the $C A D D$ method demonstrates the power of capturing both long-range dislocation plasticity and short-range atomistic phenomena in this application. The use of $C A D D$ permits for a clear study of the physical and mechanical influence of both complex plastic flow and atomistic-level processes on the macroscopic response of material as a function of temperature and rate of indenting. We have shown that the Nose-Hoover thermostat implemented in the damping band zone can serve to absorb the atomistic/continuum interface energy reflection to prevent overheating 
of the atomistic region during the indentation process. Simulations at different temperatures and velocities of indenting reveal an interesting dependency of these parameters to the first homogenous nucleation of dislocation.

For nanoindentation, the approach provides the atomistic region with the quasistatic approximation of the stress field due to long-range interaction of discrete dislocations in the continuum region. This approximation is calculated by updating of the dislocations in the continuum region on a larger time scale.

We have made a number of more quantitative observations about the deformation process than has been the case with fully atomistic simulations. Briefly, we recount the wide range of results and observations found here:

1. As has been observed by others in direct atomistic simulations $[93,91,94]$, homogeneous dislocation nucleation occurs below the surface, but not at the location of the maximum resolved shear stress on the crystal slip systems.

2. The material hardness is found to scale with indentation depth in a manner consistent with experimental studies at larger scales, suggesting that the size effect is not due to microstructural features but rather to the intrinsic evolution of the defect structure under the indentation loading.

3. Dislocation dissociation, the $2 D$ analogue of cross-slip in $3 D$, is observed underneath the indenter and plays a key role in determining the subsequent overall deformation.

4. Initial unloading is essentially elastic, despite reverse motion of previously emitted dislocations. Near complete unloading, a substantial Bauschinger effect, or change in the unloading curve slope, is observed and corresponds to the point at which dislocations begin to move out of the crystal or annihilate.

5. Upon complete unloading, only a small number of dislocations remain in the film, mainly those that had undergone dissociation and had moved laterally and near to the surface of the indented crystal.

6. The onset of plasticity or dislocation nucleation is affected by the temperature. 
Increasing temperature makes the nucleation of dislocation happen earlier.

7. The critical depths corresponding to the force-displacement curves are not the same for all velocities, thus indicating a rather noticeable dependence of the critical depth on velocity. Increasing velocity makes the nucleation of dislocation happen later.

In Chapter 5 , the dynamic $C A D D$ ability to study the material removal process by a hard circular repulsive tool from the surface of an initially defect-free aluminum single crystal. The performed simulations provide fundamental insight into the atomic details underlying nanoscale scratching of the single-crystal aluminum surface. The unique multiscale approach used here has allowed us to make a number of more-quantitative observations about the deformation process than has been the case with fully atomistic simulations.

Briefly, we summarize the results and observations achieved in this chapter:

1. As it has been observed by others in direct atomistic simulations $[109,97,98$, $99,110,111,112,113,114,115,102,103]$, dislocation generation and propagation are responsible for chip formation and surface evolution and finish.

2. Increasing the depth of scratching increases the tangential force on the tool for the same scratching speed.

3. The tangential and normal forces vary as a function of scratching speed. At scratching speeds over the propagation speed of plastic wave, the average magnitude of normal forces increases rapidly. This also corresponds to a transition from dislocation mediated plastic flow to amorphization of the crystal.

4. The chip deformation mechanism in a high speed scratching process is completely different from low speed scratching. The plastic deformation of the substrate under the tool is highly localized and the stored elastic energy is not sufficient to generate deep moving dislocations through the workpiece. By increasing the tool speed to more than $300 \mathrm{~m} / \mathrm{s}$, severe local surface plasticity and amorphization can be observed. Simulations show that the scratched surface in a high speed process is much smoother than from a low 
scratching speed.

5. Dislocation dissociation, the $2 D$ analogue of cross slip in $3 D$, is observed underneath the tool and plays a key role in determining the subsequent overall surface evolution.

6. Upon reaching the relaxed state when the tool has passed by, only a small number of dislocations remain in the workpiece, mainly those that had undergone dissociation and had moved laterally and near to the surface of the scratched crystal.

\subsection{Recommendations for future work}

Much needs to be done to make the dynamic $C A D D$ method a viable quantative tool for materials design. First, many fundamental features of the model introduced in this thesis need to be further studied and clarified.

Additional interatomic potentials should be examined. Only embedded atom potentials have been used to date with the majority of analysis carried out using the ErcolessiAdams ptentials for aluminum. It will be interesting to try potentials and compare the results with those obtained so far.

Optimization of code performance is always needed. The current implementation is somewhat optimized but is far from reaching the level of optimization common to major finite element codes and atomistic lattice molecular dynamics. As the method is applied to ever larger systems this will become key.

Another avenue for optimization lies in parallelization. The CADD method is an admirable candidate for parallelization because the atomistic summations involved in the force computations are independent and can be done in parallel. Many atomistic methods are already making use of parallel computing technology and thus $C A D D$ could profit as well.

Treatment of grain boundaries and bi-material interfaces must be introduced into the code to allow the modeling of polycrystals. Although this manuscript stresses the conceptual capabilities of $C A D D$ to treat interfaces transparently, the current implementation is 
designed to handle monocrystals alone.

In addition, the $C A D D$ method offers many exciting possibilities for enhancing the physical foundations of the theory. The first challenge lies in expanding the method to three dimensions. The current implementation is $2 D$, and while many interesting problems can be studied with it, real materials are of course three dimensional. Undoubtedly, the transition will be fraught with details and difficulties, however conceptually it appears that there are no major stumbling blocks. The atomistic computations carried out are already $3 D$ so there are no difficulties there. Three dimensional finite element codes abound, as do robust routines for three dimensional meshing. Major problems, once the three dimensional implementation is completed, will most likely be related to increased computational demands and hence an emphasis on optimization and possibly parallelization and overcoming difficulties in $3 D$ visualization and interpretation of results.

In the $3 D C A D D$ framework, handling of dislocation loops wholly contained either in the atomistic region or in the continuum region may not be a problem but the major challenge will be in handling single dislocation loops located partly in the atomistic region and partly in the continuum region.

Nonequilibrium thermomechanical behavior of solids is another possible expansion of the work and perhaps the most difficult path to tread. The literature in this field is vast and confused. It is very difficult to get a big picture of the ideas in nonequilibrium behavior. At this point, one possible direction that might be useful to explore and perhaps more challenging, is to consider the thermodynamics of the continuum region in our approach. This step is required for construction of a sound model for nonequilibrium multiscale simulations. A nonequilibrium multiscale model not only should provide a means to perform concurrent multiscale simulations of relative large system with local atomistic resolution but also should reproduce the correct underlying statistical physics. Such a multiscale formulation connects fine scale statistical mechanics to coarse scale deterministic responses. In other words, a nonequilibrium multiscale formulation should accurately capture the 
continuum mean field, and it can also provide the atomistic fluctuations in localized regions.

In closing, my experience reading articles in this area has left me with the impression that much of the work done is based on observations, and very seldom involves quantitative characterization. As far as simulations are concerned, they are getting ever bigger as computer hardware improves. It is becoming increasingly difficult to learn from these simulations, many of them resulting in merely a set of observations (like some of the simulations reported in this thesis). One problem is due to the fact that there does not seem to be good connection between discrete atomistic mechanics and continuum mechanics, of which we have a good intuitive understanding. Another problem is due to lack of accurate experimental data at the nanoscale. 


\section{Bibliography}

[1] L.E. Shilkrot, R.E. Miller, and W.A. Curtin. Multiscale plasticity modeling: Coupled atomistic and discrete dislocation mechanics. J. Mech. Phys. Sol., 52(4):755$787,2004$.

[2] I.A. Ovid'ko. Deformation of nanostructures. Science, 295(5564):2386, 2002.

[3] W.D. Nix and H. Gao. Indentaion size effects in crystalline materials: A law for strain gradient plasticity. J. Mech. Phys. Sol., 46(3):411-425, 1998.

[4] A. Szabo and N. S. Ostlund. Modern Quantum Chemistry. McGraw-Hill, New York, 1989.

[5] G. Lu and E. Kaxiras. Handbook of Theoretical and Computational Nanotechnology, chapter 22, pages 1-33. American Scientific, Stevenson Ranch, CA, 2005.

[6] M.Z. Bazant and E. Kaxiras. Modeling of covalent bonding in solids by inversion of cohesive energy curves. Phys. Rev. Lett., 77(21):4370-4373, 1996.

[7] F. Ercolessi and J. B. Adams. Interatomic potentials from first-principles calculations: The force-matching method. Europhys. Lett., 26:583-588, 1994.

[8] D. Frenkel and B. Smith. Understanding Molecular Simulation: From Algorithms to Applications. Academic Press, Boston, 2002.

[9] L.P. Kubin and G. Canova. The modelling of dislocation patterns. Scripta Metallurgica, 27:957-962, 1992. 
[10] Marc C. Fivel. Discrete dislocation dynamics: an important recent break-through in the modelling of dislocation collective behaviour. Comptes Rendus Physique, 9:427-436, 2008.

[11] T. J. R. Hughes. The Finite Element Method: Linear Static and Dynamic Finite Element Analysis. Prentice-Hall, Englewood Cliffs, N.J., 1987.

[12] O. C. Zienkiewicz. The Finite Element Method. McGraw Hill, London, 1977.

[13] Weinan E, B. Engquist, , X. Li, W. Ren, and E. Vanden-Eijnden. The heterogeneous multiscale methods: A review. Communications in Computational Physics, 2:367$450,2007$.

[14] W.A. Curtin and Ronald E. Miller. Atomistic/continuum coupling in computational material science. Mod. Simul. Mater. Sci. Eng., 11:R33--R68, 2003.

[15] J.Q. Broughton, F.F. Abraham, N. Bernstein, and E. Kaxiras. Concurrent coupling of length scales: methodology and application. Phys. Rev. B, 60:2391-2403, 1999.

[16] G. Lu, E. B. Tadmor, and E. Kaxiras. From electrons to finite elements: A concurrent multiscale approach for metals. Phys. Rev. B, 73:024108-4, 2006.

[17] R. E. Miller and E. B. Tadmor. Hybrid continuum mechanics and atomistic methods for simulating materials deformation and failure. MRS Bulletin, 32:920-926, 2007.

[18] S. A. Adelman and J. D. Doll. Generalized langevin equation approach for atom/solid-surface scattering: Collinear atom/harmonic chain model. J. Chem. Phys., 61:4242, 1974.

[19] J. D. Doll, L .E. Myers, and S. A. Adelman. Generalized langevin equation approach for atom/solid-surface scattering: Inelastic studies. J. Chem. Phys., 63:4908, 1975. 
[20] S. A. Adelman and J. D. Doll. Generalized langevin equation approach for atom/solid-surface scattering: General formalism for classical scattering off harmonic solids. J. Chem. Phys., 65:2375, 1976.

[21] Z. P. Bazant. Spurious reflection of elastic waves in nonuniform finite element grids. Comput. Methods Appl. Mech. Engrg., 16:91-100, 1978.

[22] N. Holmes and T. Belytschko. Postprocessing of finite element transient response calculations by digital filters. Computers and Structures, 6:211-216, 1976.

[23] R. E. Rudd and J. Q. Broughton. Coarse-grained molecular dynamics and the atomic limit of finite elements. Phys. Rev. B, 58(10):R5893-R5896, 1998.

[24] R. E. Rudd and J. Q. Broughton. Concurrent coupling of length scales in solid state systems. Phys. Stat. Sol. B, 217(1):251-291, 2000.

[25] R. E. Rudd. Coarse-grained molecular dynamics for computer modeling of nanomechanical systems. Intl. J. on Multiscale Comput. Engin., 2(2):203-220, 2004.

[26] R. E. Rudd and J. Q. Broughton. Coarse-grained molecular dynamics: Nonlinear finite elements and finite temperature. Phys. Rev. B, 72:144104, 2005.

[27] W. Cai, M. Koning, V. Bulatov, and S. Yip. Minimizing boundary reflections in coupled-domain simulations. Phys. Rev. Lett., 85(15):3213-3216, 2000.

[28] P. A. Deymier and J. Q. Vasseur. Concurrent multiscale model of an atomic crystal coupled with elastic continua. Phys. Rev. B, 66(13):134106, 2002.

[29] W. E and Z. Huang. Matching conditions in atomistic-continuum modeling of materials. Phys. Rev. Lett., 87(13):135501, 2001.

[30] W. E and Z. Huang. A dynamic atomistic-continuum method for the simulation of crystalline materials. J. Comput. Phys., 182(1):234-261, 2002. 
[31] T. Belytschko and S. P. Xiao. Coupling methods for continuum model with molecular model. J. Multi. Comput. Engrg., 1(1):115-126, 2003.

[32] S. P. Xiao and T. Belytschko. A bridging domain method for coupling continua with molecular dynamics. Comput. Methods Appl. Engrg., 193(17-20):1645-1669, 2004.

[33] H. S. Park and W. K. Liu. An introduction and tutorial on multiple scale analysis in solids. Computer Methods in Applied Mechanics and Engineering, 193:1733$1772,2004$.

[34] H. S. Park, E. G. Karpov, and W. K. Liu. A temperature equation for coupled atomistic/continuum simulations. Comput. Methods Appl. Mech. Engrg., 193(1720):1713-1732, 2004.

[35] G. L. Wagner and W. K. Liu. Coupling of atomistic and continuum simulations using a bridging scale decomposition. J. Computat. Phys., 190(1):249-274, 2003.

[36] W.K. Liu, H.S. Park, D. Qian, E.G. Karpov, H. Kadowaki, and G.J. Wagner. Bridging scale methods for nanomechanics and materials. Comput. Methods Appl. Mechs. Engrg., 195:1407-1421, 2006.

[37] Albert C. To and Shaofan Li. Perfectly matched multiscale simulations. Phys. Rev. $B, 72: 035414-035422,2005$.

[38] Shaofan Li, Xiaohu Liu, Ashutosh Agrawal, and Albert C. To. Perfectly matched multiscale simulations for discrete lattice systems: Extension to multiple dimensions. Phys. Rev. B, 74:045418-045432, 2006.

[39] J. P. Brenger. A perfectly matched layer for the absorption of electromagnetic waves. J. Comput. Phys., 114(1):185-200, 1994. 
[40] Xiaohu Liu and Shaofan Li. Nonequilibrium multiscale computational model. $J$. Chem. Phys., 126(12):124105, 2007.

[41] S. Qu, V. Shastry, W. A. Curtin, and R. E. Miller. A dynamic finite temperature coupled atomistic/discrete dislocation method. Modeling Simul. Mater. Sci. Eng., 13(7):1101-1118, 2005.

[42] B. Holian and R. Ravelo. Fracture simulations using large-scale molecular dynamics. Phys. Rev. B, 51(11):11275-11288, 1995.

[43] L.M. Dupuy, E.B. Tadmor, R.E. Miller, and R. Phillips. Finite temperature quasicontinuum: Molecular dynamics without all the atoms. prl, 95:060202, 2005.

[44] B. Shiari, R. E. Miller, and W. A. Curtin. Coupled atomistic/discrete dislocation dynamics simulation of nanoindentation at finite temperature. ASME Transaction, Journal of Engineering Materials and Technology, 127(4):358-368, 2005.

[45] B. Shiari, R. E. Miller, and D. D. Klug. Finite temperature multiscale computational modeling of materials at the nanoscale. In Proceedings of the 2005 International Conference on MEMS, NANO and Smart Systems, Banff, Canada, July 2005.

[46] B. Shiari, R. E. Miller, and D. D. Klug. Multiscale simulation of material removal processes at the nanoscale. J. Mech. Phys. Sol., 55(11):2384-2405, 2007.

[47] B. Shiari, R. E. Miller, W. Beres, and L. Zhao. Coupled atomisctic/continuumm discrete dislocation modeling of nanoscratching. In International Conference of Metallurgists, Montreal, Canada, Oct. 2006.

[48] N. W. Ashcroft and N. D. Mermin. Solid State Physics. Harcourt Brace, Orlando, 1976.

[49] R. Balian. From Microphysics to Macrophysics: Methods and Applications of Statistical Physics. Springer, Berlin, Germany, 1991. 
[50] Y. Hu and S. B. Sinnott. Constant temperature molecular dynamics simulations of energetic particle - solid collisions: comparison of temperature control methods. Journal of Computational Physics, 200(1):251-266, 2004.

[51] P. H. Hünenberger. Thermostat algorithms for molecular dynamics simulations. Adv. Polymer. Sci., 173:105-149, 2005.

[52] S.R.A. Salinas. Introduction to Statistical Physics. Springer, New York, 2001.

[53] S. Nosé. A molecular dynamics method for simulations in the canonical ensemble. Mol. Phys., 52:255-268, 1984.

[54] W. G. Hoover. Canonical dynamics-equilibrium phase-space distributions. Phys. Rev. A, 31:1695-1697, 1984.

[55] T. Soddemann, B. Dunweg, and K. Kremer. Dissipative particle dynamics: A useful thermostat for equilibrium and nonequilibrium molecular dynamics simulations. Physical Review E, 68:046702-8, 2003.

[56] B. B. Laird and B. J. Leimkuhler. Generalized dynamical thermostating technique. Phys. Rev. E, 68:016704-016710, 2003.

[57] O. C. Zienkiewicz. The Finite Element Method, volume 1-2. McGraw-Hill, London, 4th edition, 1991.

[58] N. Newmark. A method of computation for structural dynamics. J. Engng Mech., Div. ASCE, 85:67-94, 1959.

[59] W. C. Swope, H. C. Andersen, P. H. Berens, and K. R. Wilson. A computer simulation method for the calculation of equilibrium constants for the formation of physical clusters of molecules: Application to small water clusters. Journal of Chemical Physics, 76:637-649, 1982. 
[60] R. D. Krieg and S. W. Key. Advunces in Computational Methods in Stractural Mechanics and Design, pages 237-258. UAH Press, Huntsville, Ala., 1972.

[61] A. Streit, R. E. Zillich, P. Baumann, and P. Klein. Differentiable mesoscopic fields in molecular dynamics simulations: construction, dynamics and coupling of length scales. In Proceedings of the Multiscale Materials Modelling, Freiburg, Germany, 2006.

[62] E. B. Tadmor, M. Ortiz, and R. Phillips. Quasicontinuum analysis of defects in solids. Phil. Mag. A, 73(6):1529-1563, 1996.

[63] E. B. Tadmor, R. Phillips, and M. Ortiz. Mixed atomistic and continuum models of deformation in solids. Langmuir, 12(19):4529-4534, 1996.

[64] L.E. Shilkrot, R.E. Miller, and W.A. Curtin. Coupled atomistic and discrete dislocation plasticity. Phys. Rev. Lett., 89(2):025501-025504, 2002.

[65] M. S. Daw and M.I. Baskes. Embedded-atom method: Derivation and application to impurities, surfaces and other defects in metals. Phys. Rev. B, 29(12):6443-6453, 1984.

[66] M. S. Daw, M. I. Baskes, C. L. Bisson, and W. G. Wolfer. Application of the embedded atom method to fracture, dislocation dynamics and hydrogen embrittlement. Modeling Environmental Effects on Crack Growth Processes, pages 99-124, 1986.

[67] E. Van der Giessen and A. Needleman. Discrete dislocation plasticity: a simple planar model. Model. Simul. Mater. Sci. Eng., 3:689-735, 1995.

[68] C. S. Shin, M. C. Fivel, M. Verdier, and K. H. Oh. Dislocation-impenetrable precipitate interaction: A discrete dislocation simulation analysis. Philos. Mag., 83:3691$3704,2003$. 
[69] A. Needleman and E. Van der Giessen. Discrete dislocation plasticity. Key Engineering Materials, 233-236:13-24, 2003.

[70] V. R. Parameswaran, N. Urabe, and J. Weertman. Dislocation mobility in aluminum. J. Appl. Phys., 43(7):2982-2986, 1972.

[71] R.E. Miller, L.E. Shilkrot, and W.A. Curtin. A coupled atomistics and discrete dislocation plasticity simulation nano indentation into single crystal thin films. Acta Mat., 52(2):271-284, 2004.

[72] K. J. Van Vliet and S. Suresh. Simulations of cyclic normal indentation of crystal surfaces using the bubble-raft model. Phil. Mag. A, 82(10):1993-2001, 2002.

[73] J. P. Hirth and J. Lothe. Theory of Dislocations. McGraw-Hill, New York, 1968.

[74] A.G. Atkins and D. Tabor. Indentation measurements and elastic properties. Mech. Phys. Solids, 13:149, 1963.

[75] Bharat Bhushan and Xiaodong Li. Indentation measurements and elastic properties. International Materials Reviews, 48:125-162, 2003.

[76] Springer Handbook of Nanotechnology. Springer-Verlag, Heidelberg, Germany, 2007.

[77] J. S. Field. A simple predictive model for spherical indention. J. Mat. Res., 8:297, 1993.

[78] W. C. Oliver and G. M. Pharr. An improved technique for determining hardness and elastic modulus using load and displacement sensing indentation experiments. J. Mat. Res., 7:1564, 1992.

[79] B. Shiari and R. E. Miller. Multiscale modeling of ductile crystals at the nanoscale subjected to cyclic indentation. Acta Mat., 2008. accepted. 
[80] J. Belak, D. B. Boercker, and I. F. Stowers. Simulation of nanometerscale deformation of metallic and ceramic surfaces. MRS Bull., 18:55, 1993.

[81] D. W. Brenner, S. B. Sinnott, J. A. Harrison, and O. A. Shenderova. Simulated engineering of nanostructures. Nanotechnology, 7:161, 1996.

[82] R. Perez, M. C. Payne, and A. D. Simpson. First principles simulations of silicon nanoindentation. Phys. Rev. Lett., 75:4748, 1995.

[83] K.C. Tang and R.D. Arnell. Determination of coating mechanical properties using spherical indenters. Thin solid films, 355-356:263-269, 1999.

[84] E.T. Lilleodden, J.A. Zimmerman, S.M. Foiles, and W.D. Nix. Atomistic simulation of elastic deformation and dislocation nucleation during nanoindentation. $J$. Mech. Phys. Sol., 51:901-920, 2003.

[85] V. Vitek, R. C. Perrin, and D. K. Bowen. The core structure of $\frac{a}{2}<111>$ screw dislocations in bec crystals. Phil. Mag., 21:1049, 1970.

[86] J. K. Ternes, D. Farkas, and R. D. Kriz. Stoichiometry effects on core structure and mobility in B2 NiAl. Phil. Mag., 72:1671-1696, 1995.

[87] C. L. Kelchner, S. Plimpton, and J. C. Hamilton. Dislocation nucleation and defect structure during surface indentation. Phys. Rev. B, 58:11085-11088, 1998.

[88] J. A. Zimmerman, C. L. Kelchner, P. A. Klein, J. C. Hamiltion, and S. M. Foiles. Surface step effects on nanoindentation. Phys. Rev. Letts, 87:165507, 2001.

[89] D. Saraev and R. E. Miller. Enhanced hardness of copper crystals by nanometersized nickel coatings. Modeling Simul. Mater. Sci. Eng., 13(7):10891099, 2005.

[90] D. Saraev and R. E. Miller. Atomic-scale simulations of nanoindentation-induced plasticity in copper crystals with nanometer-sized nickel coatings. Acta Materialia, $54(1): 33-45,2006$. 
[91] R.E. Miller and A. Acharya. A stress-gradient based criterion for dislocation nucleation in crystals. Journal of the Mechanics and Physics of Solids, 52:1507-1525, 2004

[92] L.C. Kelchner, S.J. Plimpton, and J.C. Hamilton. Dislocation nucleation and defect structure during surface indentation. Phys. Rev. B, 58(17):11085-11088, 1998.

[93] K.J. Van Vliet, J. Li, T. Zhu, S. Yip, and S. Suresh. Quantifying the early stages of plasticity through nanoscale experiments and simulations. Phys. Rev. B, 67:104105, 2002.

[94] S.V. Dmitriev, J. LI, N. Yoshikawa, and Y. Shibutani. Theoretical strength of $2 d$ hexagonal crystals: application to bubble raft indentation. Philosophical Magazine, 85:2177-2195, 2005.

[95] J. Sone, J. Fujita, Y. Ochiai, S. Manako, S. Matsui, E. Nomura, T. Baba, H. Kawaura, T. Sakamoto, C. D. Chen, Y. Nakamura, and J. S. Tsai. Nanofabrication toward sub-10 $\mathrm{nm}$ and its application to novel nanodevices. Nanotechnology, 10:135-141, 1999.

[96] T. Inamura. Mechanics and engergy dissipation in nanoscale cutting. Annals of the CIRP, 42:19-23, 1993.

[97] S. Shimada, N. Ikawa, H. Tanaka, G. Ohmori, J. Uchikoshi, and H. Yoshinaga. Feasibility study on ultimate accuracy in microcutting using molecular dynamics simulation. Annals of the CIRP, 42:91-94, 1993.

[98] R. Komanduri, N. Chandrasekaran, and L. M. Raff. Orientation effects in nanometric cutting of single crystal materials: an md simulation approach. Annals of the CIRP, 48:67-70, 1999. 
[99] R. Komanduri, N. Chandrasekaran, and L. M. Raff. $M D$ simulation of nanometric cutting of single crystal aluminum-effect of crystal orientation and direction of cutting. Wear, 242:60-88, 2000.

[100] J. Shimizu, E. Ohmura, and H. Eda. Analysis of sliding mechanism using molecular dynamics: Friction process. Japanese Journal of Tribology, 41(12):1375-1386, 1996.

[101] L. Zhang and H. Tanaka. Towards a deeper understanding of wear and friction on the atomic scale: a molecular dynamics analysis. Wear, 211:44-53, 1997.

[102] K. Maekawa and A. Itoh. Friction and tool wear in nano-scale machining - a molecular dyanmics approach. Wear, 188:115-122, 1995.

[103] N. Chandrasekaran, A. Noori Khajavi, L. M. Raff, and R. Komanduri. A new method for molecular dynamics simulation of nanometric cutting. Phil. Mag. B, 77:7-26, 1998.

[104] G. M. Robinson and M. J. Jackson. A review of micro and nanomachining from a materials perspective. Journal of Materials Processing Technology, 167:316-337, 2005.

[105] L. Zhou, J. Shimizu, A. Muroya, and H. Eda. Material removal mechanism beyond plastic wave propagation rate. Precision Engineering, 27:109-116, 2003.

[106] J. Shimizu, L. B. Zhou, and H. Eda. Simulation and experimental analysis of super high-speed grinding of ductile material. J. of Material Processing Technology, 129:19-24, 2002.

[107] J. C. Tse and D. D. Klug. Mechanical instability of a-quartz: A molecular dynamics study. Phys. Rev. Lett., 67:3559-3562, 1991. 
[108] J. S. Tse, D. D. Klug, C. A. Tulk, I. Swainson, E. C. Swensson, C. K. Loong, V. Shpakov, V. R. Belosludov, R. V. Belosludov, and Y. Kawazoe. The mechanisms for pressure-induced amorphization of ice ih. Nature, 400:647-649, 1999.

[109] J. Belak, D. A. Lucca, R. Komanduri, R. L. Rhoerer, K. Moriwaki, S. Okuda nad N. Ikawa, S. Shimada, H. Tanaka, T. A. Dow, J. D. Drescher, and I. F. Stowers. Molecular dynamics simulation of the chip formation process in single crystal copper and comparison with experimental data. In Proc. ASPE Annual Conference, pages $100-109,1991$.

[110] T. H. Fang and C. I. Weng. Three-dimensional molecular dynamics analysis of processing using a pin tool on the atomic scale. Nanotechnology, 11:148-153, 2000.

[111] Z. C. Lin and J. C. Huang. A nano-orthogonal cutting model based on a modified molecular dynamics technique. Nanotechnology, 15:510-519, 2004.

[112] B. Lin, S. Y. Yu, and S. X. Wang. An experimental study on molecular dynamics simulation in nanometer grinding. J. of Material Processing Technology, 138:484$488,2003$.

[113] X. Luo, K. Cheng, X. Guo, and R. Holt. An investigation on mechanics of nanometric cutting and the development of its test-bed. Int. J. Prod. Res., 41:1449-1465, 2003.

[114] S. Jun, Y. Lee, S. Y. Kim, and S. Im. Large-scale molecular dynamics simulations of al(111) nanoscartching. Nanotechnology, 15:1169-1174, 2004.

[115] Y. D. Yan, T. Sun, S. Dong, X. C. Luo, and Y. C. Liang. Molecular dynamics simulations of processing using afm pin tool. Applied Surface Science, 2:75237531, 2005 . 